\title{
USO DE MÉTODOS BAYESIANOS NA ANÁLISE DE DADOS DE TEMPO DE VIDA BIVARIADOS
}

\author{
ROSELI APARECIDA LEANDRO \\ Mestre em Matemática Aplicada
}

Orientador: Prof. Dr. Jorge AlberTo ACHCAR

Tese apresentada à Escola Superior de Agricultura "Luiz de Queiroz", da Universidade de São Paulo, para obtenção do título de Doutor em Agronomia, Área de Concentração: Estatística e Experimentação Agronômica.

P I R A C I C A B A

Estado de São Paulo - Brasil

Fevereiro - 1997 


\section{Dados Internacionais de Catalogação na Publicação (CIP) DIVISÃO DE BIBLIOTECA E DOCUMENTAÇĀO - Campus "Luiz de Queiroz"/USP}

\section{Leandro, Roseli Aparecida}

Uso de métodos bayesianos na análise de dados de tempo de vida bivariados / Roseli Aparecida Leandro. - P Piracicaba, 1997.

179 p. : il.

Tese (doutorado) -- Escola Superior de Agricultura Luiz de Queiroz, 1997.

Bibliografia.

1. Análise estatistica 2. Inferência estatistica 3. Método de Bayes 4. Simulação computadorizada I. Titulo 


\section{USO DE MÉTODOS BAYESIANOS NA ANÁLISE DE DADOS DE TEMPO DE VIDA BIVARIADOS}

Aprovado em: 18/04/97

Comissão julgadora:

Prof. Dr. Jorge Alberto Achcar

ICMSC/USP

Prof $\underline{\underline{a}}$. Dr ${ }^{\underline{a}}$. Cicilia Wada Yuko IMECC/UNICAMP

Prof. Dr. Marinho Andrade ICMSC/USP

Prof. Dr. Heleno Bolfarine IME/USP

Prof $\underline{\underline{a}}$ Dr$^{\underline{a}}$. Clarice Garcia Borges Demétrio ESALQ/USP

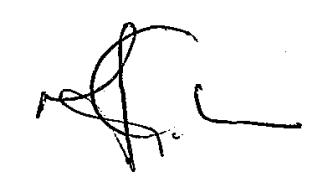

Prof. Dr. Jorge Alberto Achcar Orientador 


\section{DEDICAÇÃO}

A minha querida mãe, com eterna gratidão,

Ao meu querido pai (in memorian), com muita saudade,

A Reginaldo, Elaine, Raphael e Raíssa dedico com carinho e amor. 


\section{AGRADECIMENTOS}

Ao Professor Dr. Jorge Alberto Achcar, pela orientação e estímulo.

Aos Professores do Departamento de Matemática e Estatística da ESALQ/USP, pelo apoio.

Aos funcionários do Departamento de Matemática e Estatística da ESALQ/USP.

Aos Colegas de curso pelo companheirismo e amizade.

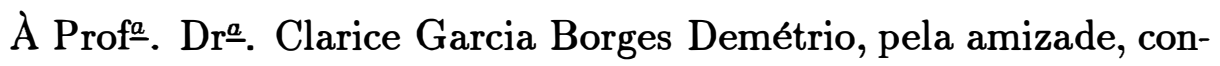
fiança e estímulo.

Aos Amigos do Coração pela força, amizade e carinho.

A todos que direta ou indiretamente, colaboraram com esta etapa da minha vida.

Ao Ser Superior que nos guia e nunca nos abandona iluminando nossos passos, DEUS. 


\section{SUMÁRIO}

1 Introdução

2 O modelo de Block \& Basu

3 Análise Clássica do Modelo de Block \& Basu 17

3.1 Estimadores de Máxima Verossimilhança . . . . . . . . . . . . 17

3.2 Inferências sobre os parâmetros $\lambda_{1}, \lambda_{2}$ e $\lambda_{3} \ldots \ldots \ldots$

3.3 Intervalos de Confiança . . . . . . . . . . . . . . . 19

3.4 Confiabilidade de Sistemas . . . . . . . . . . . . . . 20

4 Alguns aspectos sobre a simulação de dados de Tempo de vida bivariados $\quad 22$

4.1 Predicão para Y . . . . . . . . . . . . . . . . 24

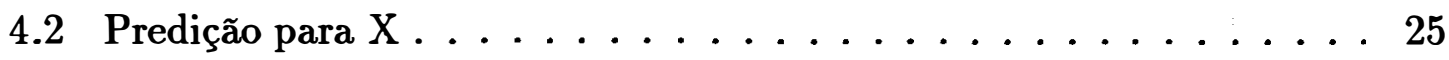

5 Aspectos de Reparametrização para a distribuição ACBVE 26

5.1 Reparametrização logarítmica . . . . . . . . . . . . . 28

5.2 Reparametrização de Box \& Cox . . . . . . . . . . . . . . . 29

5.3 Reparametrização $\left(\lambda_{1}, \lambda_{2}, \theta_{0}\right) \ldots \ldots . \ldots \ldots$

5.4 Reparametrização $\left(\lambda_{1}, \lambda_{2}, \phi_{G J}\right) \ldots \ldots \ldots \ldots$

5.5 Reparametrização Natural . . . . . . . . . . . . . . 32

5.6 Reparametrização $\left(\mu_{1}, \mu_{2}, \phi_{G J}\right) \ldots \ldots \ldots$. . . . . . . . . 34

6 Análise Bayesiana do Modelo de Block \& Basu 36

6.1 Análise Bayesiana I . . . . . . . . . . . . . 37 
6.2 Análise Bayesiana II . . . . . . . . . . . . . . . . . 39

6.2.1 Análise Bayesiana usando Gibbs com Metropolis-Hastings . . 41

6.3 Estimadores de Bayes para os tempos de vida médios . . . . . . . 45

6.4 Estimadores de Bayes para a função de Confiabilidade . . . . . . . . . 46

7 Aplicação da distribuição ACBVE em testes de vida acelerados 47

7.1 O Modelo . . . . . . . . . . . . . . . . . 48

7.2 Análise Clássica do Modelo . . . . . . . . . . . . . . . . 49

7.2.1 Estimadores de Máxima Verossimilhança . . . . . . . . . 49

7.2.2 Inferências sobre os parâmetros $c_{1}, c_{2}, c_{3} \mathrm{e} \mathcal{P} \ldots \ldots \ldots 1$

7.2 .3 Intervalos de confiança $\ldots \ldots \ldots \ldots$. . . . . . . 51

7.2 .4 Confiabilidade de Sistemas . . . . . . . . . . . . . . 52

7.3 Análise Bayesiana usando Gibbs com Metropolis-Hastings . . . . . 54

7.3.1 Estimadores de Bayes para os tempos de vida médios . . . . 56

7.3.2 Estimadores de Bayes para a função de confiabilidade . . . . . 57

8 Modelo de Regressão com Dados de Sobrevivência Bivariados 58

8.1 Análise Bayesiana usando Gibbs com Metropolis-Hastings . . . . . 61

9 Exemplos Ilustrativos $\quad 64$

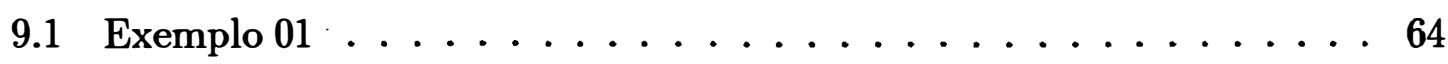

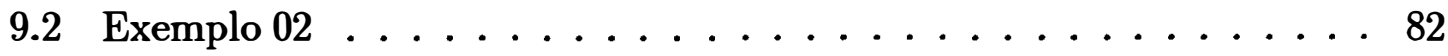

9.2.1 Análise do conjunto de dados I . . . . . . . . . . 82

9.2.2 Análise do conjunto de dados II . . . . . . . . . . 87

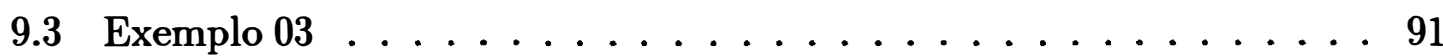

9.4 Exemplo $04 \ldots \ldots \ldots \ldots \ldots \ldots \ldots$

10 Conclusões e Perspectivas Futuras 102 
11 Bibliografia $\quad 105$

12 Apêndices $\quad 110$ 


\title{
O USO DE METODOS BAYESIANOS NA ANÁLISE DE DADOS DE TEMPO DE VIDA BIVARIADOS
}

\author{
Autora: Roseli Aparecida Leandro \\ Orientador: Prof. Dr. Jorge Alberto Achcar
}

\section{RESUMO}

Numerosos modelos paramétricos são usados na análise de dados de tempo de vida e em problemas relacionados com a modelagem de processos de falha e envelhecimento. Entre os modelos univariados, a distribuição exponencial, particularmente, tem um papel muito importante. Na prática, entretanto, o tempo de vida de um sistema depende do funcionamento de seus componentes. Nos últimos 30 anos, têm sido formuladas várias versões da distribuição exponencial bivariada para descrever o tempo de falha de um sistema com dois componentes. Neste trabalho apresenta-se um estudo sobre o Uso de Métodos Bayesianos na Análise de Dados de Sobrevivência Bivariados utilizando uma das versões da distribuição exponencial bivariada: a distribuição de Block \& BASU.

Considerando-se dados de sobrevivência bivariados e assumindo-se a distribuição de BLOCK \& BASU desenvolve-se uma análise sob o enfoque clássico e sob o enfoque Bayesiano, exploram-se diferentes parametrizações para melhorar as inferências aproximadas, realizam-se estudos sobre confiabilidade. Faz-se uma análise sob o enfoque clássico e sob o enfoque Bayesiano da aplicação da distribuição ACBVE em testes de vida acelerados. Estuda-se o modelo de regressão com dados de sobrevivência bivariados. 
A implementação prática dos métodos Bayesianos usualmente exige o uso de métodos computacionais para calcular os sumários de distribuições a posteriori de interesse. Com este fim métodos numéricos e métodos de aproximação são de grande interesse. Um método que tem sido bastante utilizado é o método de aproximação de Laplace (veja por exemplo, Tierney \& KADANe, 1986). Ocorre que, na prática, modelos que refletem a realidade são complexos ou porque apresentam um grande número de parâmetros e/ou porque a distribuição a prio$r i$ utilizada reflete realisticamente a informação a priori disponível (ou seja, não é adotada simplesmente por conveniências matemáticas). Assim tornando a aplicação destes métodos inviavél. Neste trabalho para a análise Bayesiana, utilizam-se algoritmos de simulação via Cadeias de Markov com Monte Carlo, amostrador de Gibbs (veja por exemplo, Gelfand \& SMITH, 1990) e Metropolis \& Hastings (veja por exemplo, ChIB \& Greenberg, 1995) para obter amostras aleatórias de distribuições a posteriori de interesse. 


\title{
Use of Bayesian Methods in the Analysis of Bivariate Lifetime Data
}

\author{
Autora: Roseli Aparecida Leandro \\ Orientador: Prof. Dr. Jorge Alberto Achcar
}

\section{SUMMARY}

Many parametrical models are being used in the analysis of lifetime data and in related problems with the modelling of failure and aging. Among different univariate models, the exponential distribution has been very special and important in applications, but in practical work, usually the lifetime of a system depends on different components. In the last 30 years, we observe the developing of many bivariate exponential distributions to apply in two-component systems. In this work, it is introduced the use of Bayesian methods in the analysis of bivariate exponential distribution: the bivariate exponential distribution of BLOCK \& BASU.

Considering bivariate survival data and the BLOCK \& BASU distribution, we use classical and Bayesian approaches, exploring different parametrization to improve the approximate inferences to the reliability or other functions of the parameters.

We also consider inferences for accelerated lifetime tests assuming the ACBVE distribution, and for regression models with bivariate survival data.

The pratical implementation of Bayesian methods, usually needs computational methods to get the posterior summaries of interest (approximation 
or numerical methods). A very popular method is the Laplace's method for approximation of integrals (see for example, Tierney \& KadANE, 1986). When there are many parameters and depending on the choice of the prior distribution we can have difficulties with the usual numerical or approximation methods, as is the case of the ACBVE distribution.

In this thesis, we use Markov Chain Monte Carlo methods as the Gibbs Sampling algorithm and Metropolis \& Hastings (see, for example, СНIB \& GrEenBERG, 1995) to obtain samples of the posterior distribution of interest. 


\section{Capítulo 1}

\section{Introdução}

A análise estatística do que é conhecido como dados de tempo de vida, de sobrevivência, ou tempo de falha tem tido um importante papel para pesquisadores em muitas áreas, especialmente em Engenharia e Ciências Biomédicas. As aplicações da metodologia de distribuição de tempo de vida variam da investigação da confiabilidade de ítens manufaturados à pesquisa envolvendo doenças humanas ou animais. Alguns métodos para manusear estes dados são antigos, mas muitos desenvolvimentos importantes são relativamente recentes e têm sido pesquisados e utilizados não somente por especialistas da área Estatística mas também por especialistas de outras áreas, como por exemplo, Engenharia, Medicina, etc.

Numerosos modelos paramétricos são usados na análise de dados de tempo de vida e em problemas relacionados com a modelagem de processos de falha e envelhecimento. Entre os modelos univariados, algumas distribuições particulares ocupam o papel central por causa de sua demonstração de utilidade em várias situacões. Em primeiro lugar nesta categoria estão as distribuições exponencial, Weibull, gama e log-normal.

A distribuição exponencial, particularmente, tem um papel muito importante no estudo da confiabilidade de componentes, sistemas e produtos eletrônicos em geral. Historicamente a distribuição exponencial foi o primeiro modelo 
de tempo de vida para o qual métodos estatísticos foram extensivamente desenvolvidos. Sukhatme (1937), EPSTEIn \& Sobel $(1953,1954,1955)$ e EPSTEIN $(1954,1960)$ contribuíram com vários resultados e popularizaram o uso da distribuição exponencial como modelo de tempo de vida, especialmente na área industrial.

Muitas análises de dados consideram sobrevivência como medida univariada. Mesmo os procedimentos mais complexos que consideram o uso de covariáveis (ver por exemplo, Cox, 1972) ou procedimentos de riscos competitivos (ver por exemplo, Chiang, 1970; David, 1970) ainda consideram a variável de sobrevivência de uma forma univariada.

Na prática, entretanto, o tempo de vida de um sistema depende do funcionamento de seus componentes. Este fato levou os engenheiros e biomédicos a observarem os tempos de vida do sistema e motivou os pesquisadores a tentarem formular modelos que possam descrever adequadamente os tempos de vida de sistemas com vários componentes. Um caso especial ocorre quando existem dois tempos de vida $X$ e $Y$ associados com cada sistema, em geral, correlacionados havendo a necessidade de formular modelos bivariados para descrevê-los. Um modelo muito utilizado, neste caso, é dado pela distribuição exponencial bivariada. No contexto da teoria da confiabilidade há claramente a necessidade da distribuição exponencial bivariada tanto para estudos teóricos como práticos. O efeito de qualquer correlação entre variáveis na confiabilidade total de um sistema é de grande interesse e atualmente informação nessa área é extremamente limitada. Diferentemente da distribuição normal não existe uma extensão natural única para a distribuição exponencial univariada. Nos últimos 30 anos, têm sido formuladas várias versões da distribuição exponencial bivariada para descrever o tempo de falha de um sistema com dois componentes. Entre essas versões podem-se mencionar as distribuições de Gumbel (1960), Freund (1961), Marshall \& Olkin (1967), Downton (1970), Hawkes (1972), Block \& Basu (1974) e Sarkar (1987).

Apresentam-se a seguir algumas distribuições exponenciais bivaria- 
das existentes na literatura.

Inicialmente, GUMBEL (1960) estuda algumas distribuições bivariadas com marginais exponenciais e adverte que uma distribuição bivariada não é determinada pelo conhecimento das marginais visto que dadas as distribuições marginais existem muitas distribuições bivariadas com essas marginais. GUMBEL propõe os três modelos a seguir:

Modelo 1: A distribuição exponencial bivariada tem função de distribuição conjunta dada por:

$$
F_{X, Y}(x, y)=1-e^{-x}-e^{-y}+e^{-(x+y+\delta x y)}
$$

onde $x, y \geq 0,0<\delta \leq 1$ e cuja função de densidade conjunta é dada por

$$
f_{X, Y}(x, y)=e^{-x(1+\delta y)-y}[(1+\delta x)(1+\delta y)-\delta]
$$

e as funções de distribuições marginais são exponenciais e dadas por:

$$
F_{X}(x)=1-e^{-x}, \quad F_{Y}(y)=1-e^{-y}
$$

Nota: $\mathrm{O}$ caso $\delta=0$ implica independência entre $X$ e $Y$.

$\mathrm{O}$ valor esperado do produto cruzado $X Y$ é dado por:

$$
E(X Y)=\delta^{-1} \exp (1 / \delta) K
$$

onde

$$
K=\int_{\delta^{-1}}^{\infty} \frac{e^{-z}}{z} d z
$$

Portanto, o coeficiente de correlação linear entre $X$ e $Y$ é dado por:

$$
\rho=\delta^{-1} \exp (-1 / \delta) K-1
$$


MODELo 2: No segundo modelo proposto a distribuição exponencial bivariada é um caso especial da distribuição bivariada de MorgensTERN (1956), o qual mostra que dadas duas funções de distribuições: $F_{X}(x)$ e $F_{Y}(y)$ uma função de distribuição conjunta bivariada $F_{X, Y}$ pode ser construída como sendo

$$
F_{X, Y}(x, y)=F_{X}(x) F_{Y}(y)\left[1+\alpha\left(1-F_{X}(x)\right)\left(1-F_{Y}(y)\right)\right]
$$

onde $x, y \geq 0,|\alpha| \leq 1$. Assim, Gumbel considerando $F_{X}(x)$ e $F_{Y}(y)$ funções de distribuições exponenciais propõe uma distribuição exponencial bivariada com função de distribuição conjunta dada por:

$$
F_{X, Y}=\left(1-e^{-x}\right)\left(1-e^{-y}\right)\left(1+\alpha e^{-x-y}\right)
$$

onde $x, y \geq 0,|\alpha| \leq 1$.

A função densidade de probabilidade é dada por:

$$
f_{X, Y}(x, y)=e^{-x-y}\left[1+\alpha\left(2 e^{-x}-1\right)\left(2 e^{-y}-1\right)\right]
$$

Nota 1: $X$ e $Y$ são independentes quando $\alpha=0$.

Nota 2: As funções densidades marginais são exponenciais.

O valor esperado do produto cruzado $X Y$ é dado por:

$$
E(X Y)=1+\frac{\alpha}{4}
$$

Então, o coeficiente de correlação linear entre $X$ e $Y$, é dado por:

$$
\rho(X, Y)=\frac{\alpha}{4}
$$

MODELO 3: No terceiro modelo proposto a função distribuição conjunta é dada por:

$$
F_{X Y}(x y)=1-e^{-x}-e^{-y}+p(x, y)
$$


onde $x, y \geq 0, p(x, y)=\exp \left[-\left(x^{m}+y^{m}\right)^{1 / m}\right]$ e $m \geq 1$

A função densidade conjunta para $X$ e $Y$ é dada por:

$$
f_{X, Y}(x, y)=p(x, y)\left(x^{m}+y^{m}\right)^{(1 / m-2)} x^{m-1} y^{m-1}\left[\left(x^{m}+y^{m}\right)^{1 / m}+m-1\right]
$$

GUMBEL (1960) propõe diferentes modelos mas não discute a conveniência desses modelos em situações físicas particulares. FREUND (1961), apresenta uma extensão bivariada diferente da distribuição exponencial que é especificada, em particular, para o teste de vida de um sistema com dois componentes, que pode funcionar mesmo depois que um dos componentes tenha falhado. Pode assim, ser aplicado ao estudo da falha de motores de aviões com dois motores, a performance de olhos, ouvidos, rins e outros órgãos pareados. Diferentemente do modelo proposto por GUMBEL, as funções de distribuições marginais de FREUND não são em geral exponenciais. Para a dedução do modelo Freund assume um sistema com dois componentes A e B sendo $X$ e $Y$ os respectivos tempos de vida os quais possuem distribuição exponencial com parâmetros $\alpha$ e $\beta$, respectivamente e são independentes. Se o componente A falhar primeiro o componente B sofre uma sobrecarga (ou estresse) a qual acarreta uma mudança no parâmetro da distribuição do tempo de vida do componente $\mathrm{B}$ de $\beta$ para $\beta^{\prime}$. De maneira analóga, se ocorrer falha no componente $\mathrm{B}$, o parâmetro da distribuição de tempo de vida do componente $\mathrm{A}$ muda de $\alpha$ para $\alpha^{\prime}$. Sob estas hipotéses, FREUND propõe a seguinte função de densidade conjunta para $X$ e $Y$ :

$$
f_{X, Y}(x, y)= \begin{cases}\alpha \beta^{\prime} \exp \left[-\beta^{\prime} y-\left(\alpha+\beta-\beta^{\prime}\right) x\right] & \text { se } 0<x<y \\ \alpha^{\prime} \beta \exp \left[-\alpha^{\prime} x-\left(\alpha+\beta-\alpha^{\prime}\right) y\right] & \text { se } 0<y<x\end{cases}
$$

onde $\alpha, \beta, \alpha^{\prime}, \beta^{\prime}>0$.

As funções densidades marginais considerando-se o modelo (1.1) 
não são exponenciais e são dadas por:

$$
\begin{aligned}
& f_{X}(x)=\frac{\left(\alpha-\alpha^{\prime}\right)(\alpha+\beta) e^{-(\alpha+\beta) x}}{\alpha+\beta-\alpha^{\prime}}+\frac{\alpha^{\prime} \beta e^{-\alpha^{\prime} x}}{\alpha+\beta-\alpha^{\prime}} \quad \operatorname{com} \alpha+\beta \neq \alpha^{\prime} \\
& f_{X}(x)=\frac{\left(\beta-\beta^{\prime}\right)(\alpha+\beta) e^{-(\alpha+\beta) y}}{\alpha+\beta-\beta^{\prime}}+\frac{\alpha \beta^{\prime} e^{-\beta^{\prime} y}}{\alpha+\beta-\beta^{\prime}} \quad \operatorname{com} \alpha+\beta \neq \beta^{\prime}
\end{aligned}
$$

A função geradora de momentos da distribuição exponencial bivariada (1.1) é dada por:

$$
m(s, t)=\frac{1}{\alpha+\beta-s-t}\left[\frac{\beta}{1-s / \alpha^{\prime}}+\frac{\alpha}{1-t / \beta^{\prime}}\right]
$$

e o coeficiente de correlação linear entre $X$ e $Y$ é dado por:

$$
\rho(x, y)=\frac{\alpha^{\prime} \beta^{\prime}-\alpha \beta}{\left[\left(\alpha^{\prime 2}+2 \alpha \beta+\beta^{2}\right)\left(\beta^{\prime 2}+2 \alpha \beta+\alpha^{2}\right)\right]^{1 / 2}}
$$

Deve-se observar que:

a) Em muitas aplicações $\alpha^{\prime}>\alpha, \beta^{\prime}>\beta$, isto é, o tempo de vida tende a ser pequeno quando o outro não está funcionando.

b) Quando $\alpha^{\prime} \longrightarrow \infty$ e $\beta^{\prime} \longrightarrow \infty$ tem-se $\rho \longrightarrow 1$; isto significa que um sistema de dois componentes não pode funcionar se qualquer um dos dois componentes falhar.

c) $\mathrm{O}$ coeficiente de correlação tende a $-1 / 3$ quando $\alpha=\beta, \alpha^{\prime} \longrightarrow 0$ e $\beta^{\prime} \longrightarrow 0$; isto significa que qualquer componente torna-se "quase infalível" quando o outro componente falhar. Esta não seria uma situação realista.

Marshall \& OlKIN (1967) propõem uma distribuição exponencial bivariada muito importante a distribuição BVE. Esta distribuição preserva, nas duas dimensões, a propriedade de que a vida residual é independente da idade e foi obtida assumindo que falhas podem ser causadas por três tipos de "choques" de Poisson em um sistema contendo dois componentes. Esses choques são mutuamente independentes, ocorrem a taxas $\lambda_{1}, \lambda_{2}, \lambda_{12}$ causando falhas no primeiro componente, no segundo e em ambos os componentes respectivamente. 
Modelo de Choques fatais: Suponha que os componentes de um sistema de dois componentes falhem depois de receber um choque que é sempre fatal, e que processos de Poisson independentes $Z_{1}\left(t, \lambda_{1}\right)$, $Z_{2}\left(t, \lambda_{2}\right)$ e $Z_{12}\left(t, \lambda_{12}\right)$ governem a ocorrência de choques, onde eventos no processo $Z_{1}\left(t, \lambda_{1}\right)$ são choques no componente $1, Z_{2}\left(t, \lambda_{2}\right)$ no componente 2 e $Z_{12}\left(t, \lambda_{12}\right)$ nos componentes 1 e 2 simultaneamente. Se $X$ e $Y$ denotam os tempos de vida dos componentes 1 e 2 respectivamente, tem-se:

$$
\begin{aligned}
\bar{F}_{X, Y}(s, t) & =P[X>s, Y>t] \\
& =P\left[Z_{1}\left(s, \lambda_{1}\right)=0, Z_{2}\left(t, \lambda_{2}\right)=0, Z_{12}\left(\max (s, t), \lambda_{12}\right)=0\right) \\
& =\exp \left(-\left(\lambda_{1} s-\lambda_{2} t-\lambda_{12} \max (s, t)\right)\right]
\end{aligned}
$$

Modelo DE CHOQUeS NÃo fatais: Suponha um sistema de dois e três processos de Poisson independentes $Z_{1}\left(t, \lambda_{1}\right), Z_{2}\left(t, \lambda_{2}\right)$ e $Z_{12}\left(t, \lambda_{12}\right)$ governando a ocorrência de choques que não precisam ser fatais. Sendo o estado do sistema descrito pelos pares ordenados: $(0,0),(0,1),(1,0)$, $(1,1)$, onde " 1 " indica que um componente está funcionando e " 0 " indica que não está. Suponha que eventos no processo $Z_{1}\left(t, \lambda_{1}\right)$ são choques no primeiro componente que causam uma transição de $(1,1)$ até $(0,1)$ com probalidade $p_{1}$ e de $(1,1)$ até $(1,1)$ com probalidade $\left(1-p_{1}\right)$. Analogamente, eventos no processo $Z_{2}\left(t, \lambda_{2}\right)$ são transições de $(1,1)$ até $(1,0)$ ou $(1,1)$ com probabilidade $p_{2}$ e $\left(1-p_{2}\right)$, respectivamente. Eventos no processo $Z_{3}\left(t, \lambda_{12}\right)$ são choques nos dois componentes que causam uma transição do estado $(1,1)$ até os estados $(0,0),(0,1),(1,0),(1,1)$ com probabilidades $p_{00}, p_{01}, p_{10}$ e $p_{11}$, respectivamente.Além disso assumindo que cada choque em um componente representa uma chance independente de falha e $X$ e $Y$ representam os tempos de vida dos componente 1 e 2 respectivamente, então: 


$$
\begin{gathered}
P[X>s, Y>t]=e^{\delta_{1} s-\delta_{2} t-\delta_{3} \max (s, t)} \\
\text { onde } \delta_{1}=\lambda_{1} p_{1}+\lambda_{12} p_{01}, \delta_{2}=\lambda_{2} p_{2}+\lambda_{12} p_{10} \quad \text { e } \quad \delta_{3}=\lambda_{12} p_{00}
\end{gathered}
$$

Embora esse modelo possa descrever algumas situações físicas (isto é, como os tempos de vida dos motores de aviões) ele oferece dificuldades matemáticas: apresenta uma parte singular e uma parte contínua. A singularidade é causada pelo fato de que há uma probabilidade não nula de que os tempos de vida dos dois componentes sejam iguais. Em aplicações práticas, onde dados observados são disponíveis, seria claro quando o modelo de MARShall \& OLKIN é apropriado,desde que pares de observações idênticas podem aparecer. Esse modelo, entretanto pode não ser apropriado em situações mais usuais, onde a falha de um componente enfraquece um segundo, diminuindo assim sua vida sem causar um falha catastrófica ou em certos tipos de doenças onde a falha simultânea de um par de orgãos é rara.

Ainda, o fato do modelo de MARshall \& OlKin preservar em duas dimensões a falta de memória da distribuição univariada parece uma vantagem questionável. Em um sistema com dois componentes, por exemplo, correlação pode frequentemente surgir porque um componente possui em algum sentido memória do tempo de falha do outro.

Como foi mencionado, apesar de a distribuição de MARshall \& OLKIN ser bem aceita devido ao fato de ter algumas propriedades úteis, ela não é apropriada em situações onde os dois componentes não podem falhar simultaneamente com probabilidade positiva pois se $X$ e $Y$ têm distribuição BvE então $X=Y$ tem probabilidade positiva. Esta propriedade torna a distribuição BvE inapropriada em casos onde a probabilidade de ocorrência de falha simultânea é rara. Assim uma distribuição exponencial bivariada apropriada para descrever o tempo de falha desses órgãos pareados poderia ser uma distribuição exponencial bivariada absolutamente contínua que conseguisse manter as "boas" propriedades da distribuição BVE 
de Marshall \& Olkin. Com esse objetivo Block \& Basu (1974) apresentam duas extensões bivariadas absolutamente contínuas da distribuição exponencial. Com o objetivo de obter uma distribuição bivariada absolutamente contínua com a propriedade da perda de memória eles sugeriram que as distribuições marginais fossem misturas ou médias ponderadas de exponenciais (visto que foi mostrado por MARShALL \& OLKIN que assumindo marginais exponenciais e a propriedade da perda de memória a distribuição Bve é obtida), usando as marginais propostas e a distribuição Bve de Marshall \& OlKIN como base, Block \& BASU obtiveram a distribuição bivariada a qual designaram por ACBVE. A distribuição ACBVE é a parte absolutamente contínua da distribuição BVE de MARShall \& Olkin assim como, uma variante da distribuição de Freund.

Downton (1970) deduz uma distribuição bivariada utilizando um modelo de danos sucessivos. Considera um sistema de dois componentes cada um dos quais está sujeito a choques não fatais governados por processos de Poisson. Assume que o $i$-ésimo componente falha depois de $N_{i}$ choques $\left(N_{i}\right.$ segue uma distribuição geométrica).

HAW KES (1972) usando diferentes distribuições geométricas bivariadas e utilizando o mesmo modelo de Downton obtém uma distribuição mais geral.

SARKAR (1987) deduz uma distribuição bivariada absolutamente contínua com marginais exponenciais que não possui a propriedade da perda da memória mas é semelhante à distribuição Bve de Marshall \& Olkin. Para sua dedução SARKAR modificou apenas uma propriedade de caracterização da distribuição Bve de Marshall \& OlKin demonstrada por Block (1977).

A propriedade de caracterização importante da variável aleatória da distribuição Bve $(X, Y)$, provada por Block (1977), é que os seguintes resultados são verdadeiros:

a) $X$ e $Y$ possuem funções densidade marginais exponenciais 
b) $\min (X, Y)$ é exponencial

c) $\min (X, Y)$ é independente de $X-Y$.

Esta propriedade de caracterização é modificada somente na condição (c), a qual é substituída pela condição de indepedência entre $\min (X, Y)$ e alguma função $g(X, Y)$, que como $X-Y$ na distribuição Bve, é tal que $g(X, X)=0, \mathrm{~g}(\mathrm{X}, \mathrm{Y})$ é estritamente crescente (decrescente) em $X(Y)$ para $Y(X)$ fixo. Assume também que $g(X, Y)$ possui uma distribuição especificada.

Neste trabalho apresenta-se um estudo sobre o Uso de Métodos Bayesianos na Análise de dados de Sobrevivência Bivariados utilizando uma das versoẽs da distribuição exponencial bivariada: a distribuição exponencial bivariada de BLOCK \& BASU. Considerando-se dados de sobrevivência bivariados e assumindo-se a distribuição de BLOCK \& BASU pretende-se:

1. Desenvolver uma análise sob o enfoque clássico e sob o enfoque Bayesiano.

2. Explorar diferentes parametrizações para melhorar as inferências aproximadas nos enfoques clássico e Bayesiano.

3. Estudar processos de simulação para a distribuição ACBVE.

4. Realizar estudos de confiabilidade sob o enfoque clássico e Bayesiano.

5. De forma similar, desenvolver uma análise Bayesiana para um modelo de regressão.

6. Para a análise Bayesiana, utilizarem-se algoritmos de simulação via Cadeias de Markov com Monte Carlo, por exemplo o amostrador de Gibbs (ver por exemplo, Gelfand \& SMIth, 1990) e o algoritmo de Metropolis \& Hastings (ver por exemplo, Chib \& Green Berg, 1995).

7. Desenvolver programas computacionais para geração de dados da distribuição ACBVE e para análise Bayesiana, usando o amostrador de Gibbs. 


\section{Capítulo 2}

\section{O modelo de Block \& Basu}

A distribuição exponencial bivariada (ACBVE) de BLOCK \& BASU com parâmetros $\lambda_{1}, \lambda_{2}$ e $\lambda_{3}$, para os tempos de vida $X$ e $Y$ tem função de densidade conjunta dada por

$$
f(x, y)= \begin{cases}f_{1}(x, y)=\frac{\lambda \lambda_{1} \lambda_{23}}{\lambda_{12}} \exp \left\{-\lambda_{1} x-\lambda_{23} y\right\} & \text { se } x<y \\ f_{2}(x, y)=\frac{\lambda \lambda_{2} \lambda_{13}}{\lambda_{12}} \exp \left\{-\lambda_{13} x-\lambda_{2} y\right\} & \text { se } x>y\end{cases}
$$

onde

$$
\lambda_{12}=\lambda_{1}+\lambda_{2}, \lambda_{13}=\lambda_{1}+\lambda_{3}, \lambda_{23}=\lambda_{2}+\lambda_{3} \text { e } \lambda=\lambda_{1}+\lambda_{2}+\lambda_{3} .
$$

A função geradora de momento para a ACBVE é dada por

$$
m(s, t)=E\left(e^{s X+t Y}\right)=\frac{\lambda}{\lambda_{12}(\lambda-t-s)}\left\{\frac{\lambda_{1} \lambda_{23}}{\lambda_{23}-t}+\frac{\lambda_{2} \lambda_{13}}{\lambda_{13}-s}\right\}
$$

De (2.2), obtemos os momentos de interesse para $X$ e $Y$; assim, a média e a variância para $X$ e $Y$ são dadas por: 


$$
\begin{gathered}
E(X)=\frac{1}{\lambda_{13}}+\frac{\lambda_{2} \lambda_{3}}{\lambda \lambda_{12} \lambda_{13}} \\
E(Y)=\frac{1}{\lambda_{23}}+\frac{\lambda_{1} \lambda_{3}}{\lambda \lambda_{12} \lambda_{23}} \\
\sigma_{X}^{2}=\operatorname{var}(X)=\frac{1}{\lambda_{13}^{2}}+\frac{\lambda_{2} \lambda_{3}\left(2 \lambda_{1} \lambda+\lambda_{2} \lambda_{3}\right)}{\lambda^{2} \lambda_{12}^{2} \lambda_{13}^{2}} \\
\sigma_{Y}^{2}=\operatorname{var}(Y)=\frac{1}{\lambda_{23}^{2}}+\frac{\lambda_{1} \lambda_{3}\left(2 \lambda_{2} \lambda+\lambda_{1} \lambda_{3}\right)}{\lambda^{2} \lambda_{12}^{2} \lambda_{23}^{2}}
\end{gathered}
$$

O coeficiente de correlação para $X$ e $Y$ é dado por

$$
\rho_{X Y}=\frac{\lambda_{3}\left[\left(\lambda_{1}^{2}+\lambda_{2}^{2}\right) \lambda+\lambda_{1} \lambda_{2} \lambda_{3}\right]}{\phi_{1} \phi_{2}}
$$

onde

$$
\begin{aligned}
& \phi_{1}=\left[\lambda_{12}^{2} \lambda_{13}^{2}+\lambda_{2}\left(\lambda_{2}+2 \lambda_{1}\right) \lambda^{2}\right]^{1 / 2} \\
& \phi_{2}=\left[\lambda_{12}^{2} \lambda_{23}^{2}+\lambda_{1}\left(\lambda_{1}+2 \lambda_{2}\right) \lambda^{2}\right]^{1 / 2}
\end{aligned}
$$

Observe que $0 \leq \rho_{X Y} \leq 1$ e $\rho_{X Y}=0$ somente para o caso trivial $\lambda_{3}=0$ ou $\lambda_{1}=\lambda_{2}=0$.

Algumas propriedades da distribuição ACBVE são dadas por,

1. $W=\min (X, Y)$ tem distribuição exponencial com média $1 / \lambda, \lambda=\lambda_{1}+\lambda_{2}+\lambda_{3}$.

2. $Z=X-Y$ tem função de distribuição ,

$$
F(z)= \begin{cases}\frac{\lambda_{1}}{\lambda_{12}} e^{\lambda_{23} z} & \text { se } z \leq 0 \\ 1-\frac{\lambda_{2}}{\lambda_{12}} e^{-\lambda_{13 z}} & \text { se } z>0\end{cases}
$$


3. $Z=|X-Y|$ tem função densidade dada por:

$$
\begin{aligned}
F(z)= & \frac{\lambda_{1}}{\left(\lambda_{1}+\lambda_{2}\right)}\left(1-\exp \left[-\left(\lambda_{2}+\lambda_{12}\right) z\right]\right) \\
& +\frac{\lambda_{2}}{\left(\lambda_{1}+\lambda_{2}\right)}\left(1-\exp \left[-\left(\lambda_{1}+\lambda_{12}\right) z\right]\right) \quad \text { se } z>0 .
\end{aligned}
$$

4. $W=\min (X, Y)$ é independente de $Z=X-Y$ ( e também de $|X-Y|)$.

5. A propriedade de perda de memória é válida.

6. Se $(X, Y)$ tem distribuição ACBVE, então,

$$
\begin{aligned}
& P(X>x)=\frac{\lambda}{\lambda_{12}} e^{-\lambda_{13} x}-\frac{\lambda_{3}}{\lambda_{12}} e^{-\lambda x}, \quad x>0 \\
& P(Y>y)=\frac{\lambda}{\lambda_{12}} e^{-\lambda_{23} y}-\frac{\lambda_{3}}{\lambda_{12}} e^{-\lambda y}, \quad y>0
\end{aligned}
$$

Observe que se $(X, Y)$ tem distribuição ACBVE com densidade (2.1), as variáveis aleatórias $X$ e $Y$ não são exponencialmente distribuŕdas. A função densidade de probabilidade para $X$ e $Y$ são dadas por

$$
\begin{aligned}
& f_{X}(x)=\frac{\lambda}{\lambda_{12}}\left\{\lambda_{13} e^{-\lambda_{13} x}-\lambda_{3} e^{-\lambda x}\right\}, \quad x>0 \\
& f_{Y}(y)=\frac{\lambda}{\lambda_{12}}\left\{\lambda_{23} e^{-\lambda_{23} y}-\lambda_{3} e^{-\lambda y}\right\}, \quad y>0
\end{aligned}
$$

Usualmente as inferências para esse modelo são feitas usando procedimentos clássicos via teoria assintótica dos estimadores de máxima verossimilhança (ver por exemplo, LAWLESS, 1982). Uma análise Bayesiana para este modelo foi introduzida por ACHCAR \& SANTANDER (1993), usando métodos de aproximação 
de Laplace (ver por exemplo, TIERneY \& KADANE, 1986) para a obtenção das quantidades a posteriori de interesse. 


\section{Capítulo 3}

\section{Análise Clássica do Modelo de}

\section{Block \& Basu}

\subsection{Estimadores de Máxima verossimilhança}

Os estimadores de máxima verossimilhança (EMV) para os parâmetros $\lambda_{1}, \lambda_{2}, \lambda_{3}$ são os valores $\hat{\lambda}_{1}, \hat{\lambda}_{2}, \hat{\lambda}_{3}$ que maximizam a função de verossimillhança

$$
L\left(\lambda_{1}, \lambda_{2}, \lambda_{3}\right)=\frac{\lambda_{1}^{r} \lambda^{n} \lambda_{2}^{n-r} \lambda_{23}^{r} \lambda_{13}^{n-r}}{\lambda_{12}^{n}} \exp \left\{-\lambda_{1} n \bar{x}-\lambda_{2} n \bar{y}-\lambda_{3} R m\right\}
$$

onde $r=\sum_{i=1}^{n} \delta_{i}, \quad R m=\sum_{i=1}^{n}\left[\delta_{i} y_{i}+\left(1-\delta_{i}\right) x_{i}\right], \quad n \bar{x}=\sum_{i=1}^{n} x_{i}, \quad n \bar{y}=\sum_{i=1}^{n} y_{i}$ e

$$
=\left\{\begin{array}{c}
1, X_{i}<Y_{i} \\
0, X_{i} \geq Y_{i}
\end{array}\right.
$$

Como a função logarítmica natural é uma função monotóna, então, encontrar o ponto que maximiza $L\left(\lambda_{1}, \lambda_{2}, \lambda_{3}\right)$ é equivalente a encontrar o ponto que 
maximiza o logaritmo da função de verossimilhança, isto é, que maximiza:

$$
\begin{gathered}
l\left(\lambda_{1}, \lambda_{2}, \lambda_{3}\right)=\ln L\left(\lambda_{1}, \lambda_{2}, \lambda_{3}\right) \\
l\left(\lambda_{1}, \lambda_{2}, \lambda_{3}\right)=r \ln \lambda_{1}+(n-r) \ln \lambda_{2}+n \ln \lambda+r \ln \lambda_{23} \\
+(n-r) \ln \lambda_{13}-n \ln \lambda_{12}-\lambda_{1} n \bar{x}-\lambda_{2} n \bar{y}-\lambda_{3} R m
\end{gathered}
$$

Considerando-se a equação (3.3) tem-se que as derivadas parciais de primeira ordem do logaritmo da função verossimilhança para $\lambda_{1}, \lambda_{2}$ e $\lambda_{3}$ são dadas por:

$$
\begin{aligned}
& \frac{\partial l\left(\lambda_{1}, \lambda_{2}, \lambda_{3}\right)}{\partial \lambda_{1}}=\frac{r}{\lambda_{1}}+\frac{n}{\lambda}+\frac{n-r}{\lambda_{13}}-\frac{n}{\lambda_{12}}-n \bar{x} \\
& \frac{\partial l\left(\lambda_{1}, \lambda_{2}, \lambda_{3}\right)}{\partial \lambda_{2}}=\frac{n-r}{\lambda_{2}}+\frac{n}{\lambda}+\frac{r}{\lambda_{23}}-\frac{n}{\lambda_{12}}-n \bar{y} \\
& \frac{\partial l\left(\lambda_{1}, \lambda_{2}, \lambda_{3}\right)}{\partial \lambda_{3}}=\frac{n}{\lambda}+\frac{r}{\lambda_{23}}+\frac{n-r}{\lambda_{13}}-R m
\end{aligned}
$$

Resolvendo-se o sistema de equações simultâneas (3.5) para $\lambda_{1}, \lambda_{2}$ e $\lambda_{3}$ encontram-se os EMV,

$$
\left\{\begin{array}{l}
\frac{\partial l\left(\lambda_{1}, \lambda_{2}, \lambda_{3}\right)}{\partial \lambda_{1}}=0 \\
\frac{\partial l\left(\lambda_{1}, \lambda_{2}, \lambda_{3}\right)}{\partial \lambda_{2}}=0 \\
\frac{\partial l\left(\lambda_{1}, \lambda_{2}, \lambda_{3}\right)}{\partial \lambda_{3}}=0
\end{array}\right.
$$

Visto que não é possível encontrar uma forma analítica fechada para $\lambda_{1}, \lambda_{2}$ e $\lambda_{3}$ deve-se empregar um método numérico para resolver o sistema de 
equações simultâneas (3.5). Um método bastante utilizado é o método de NewtonRaphson (ver por exemplo, LEE, 1992) .

\subsection{Inferências sobre os parâmetros $\lambda_{1}, \lambda_{2}$ e $\lambda_{3}$.}

Para fazer inferências sobre os parâmetros, utiliza-se a aproximação normal assintótica dos estimadores de máxima verossimilhança dada por:

$$
\left(\hat{\lambda_{1}}, \hat{\lambda_{2}}, \hat{\lambda_{3}}\right) \stackrel{a}{\sim} N_{3}\left[\left(\lambda_{1}, \lambda_{2}, \lambda_{3}\right) ; \mathcal{I}^{-1}\right]
$$

onde $\mathcal{I}$ é a matriz de informação observada, isto é, a matriz das derivadas parciais de segunda ordem do logaritmo da função de verossimilhança $l\left(\lambda_{1}, \lambda_{2}, \lambda_{3}\right)$ multiplicados por $(-1)$.

\subsection{Intervalos de Confiança}

Considerando a normalidade assintótica dos estimadores de máxima verossimilhança $\hat{\lambda_{1}}, \hat{\lambda_{2}}$ e $\hat{\lambda_{3}}$ podem-se calcular os intervalos de confiança para os parâmetros $\lambda_{1}, \lambda_{2}$ e $\lambda_{3}$.

Representando a inversa da matriz de informação observada por:

$$
\mathcal{I}^{-1}=\left[\begin{array}{lll}
b_{11} & b_{12} & b_{13} \\
b_{21} & b_{22} & b_{23} \\
b_{31} & b_{32} & b_{33}
\end{array}\right]
$$

e considerando um nível de confiança $100(1-\alpha) \%$, os intervalos de confiança para $\lambda_{1}, \lambda_{2}$ e $\lambda_{3}$ são dados por:

$$
i c\left(\lambda_{1}\right)=\left[\hat{\lambda}_{1}-z_{\alpha / 2}\left(\hat{b}_{11}\right)^{1 / 2} ; \hat{\lambda}_{1}+z_{\alpha / 2}\left(\hat{b}_{11}\right)^{1 / 2}\right]
$$




$$
\begin{aligned}
& i c\left(\lambda_{2}\right)=\left[\hat{\lambda_{2}}-z_{\alpha / 2}\left(\hat{b}_{22}\right)^{1 / 2} ; \hat{\lambda_{2}}+z_{\alpha / 2}\left(\hat{b}_{22}\right)^{1 / 2}\right] \\
& i c\left(\lambda_{3}\right)=\left[\hat{\lambda_{3}}-z_{\alpha / 2}\left(\hat{b}_{33}\right)^{1 / 2} ; \hat{\lambda_{3}}+z_{\alpha / 2}\left(\hat{b}_{33}\right)^{1 / 2}\right]
\end{aligned}
$$

onde $z_{\alpha / 2}$ é o quantil correspondente da distribuição normal padronizada, e $\hat{b}_{i j}$ é o elemento da inversa da matriz de informação observada $\mathcal{I}$ avaliado nos estimadores de máxima verossimilhança.

\subsection{Confiabilidade de sistemas}

Se os tempos de vida de um sistema de dois componentes seguem uma distribuição com densidade (2.1), os tempos médios de vida $\mu_{1}=E(X)$ e $\mu_{2}=E(Y)$ desses componentes são dados por:

$$
\begin{aligned}
& \mu_{1}=\frac{\lambda \lambda_{12}+\lambda_{2} \lambda_{3}}{\lambda \lambda_{12} \lambda_{13}} \\
& \mu_{2}=\frac{\lambda \lambda_{12}+\lambda_{1} \lambda_{3}}{\lambda \lambda_{12} \lambda_{23}}
\end{aligned}
$$

conforme já mostrado em (2.3).

Assumindo-se um sistema composto por dois componentes, ligados em série, tem-se que o sistema funciona se os dois componentes funcionam simultaneamente; assumindo-se, ainda, que eles têm distribuição de vida com função de densidade dada por (2.1), a função de confiabilidade do sistema no tempo $t_{0}$ é dada por:

$$
R_{S}\left(t_{0}\right)=P\left[\min (X, Y)>t_{0}\right]=e^{-\lambda t_{0}}
$$


Considerando-se o mesmo sistema onde os componentes estão ligados em paralelo e possuem distribuição de vida com função de densidade (2.1), a função de confiabilidade do sistema no tempo $t_{0}$ é dada por:

$$
R_{P}\left(t_{0}\right)=P\left[\max (X, Y)>t_{0}\right]=\frac{\lambda\left(e^{\lambda_{1} t_{0}}+e^{\lambda_{2} t_{0}}-1\right)-\lambda_{3}}{\lambda_{12} e^{\lambda t_{0}}}
$$

Em geral, as inferências para a confiabilidade de sistemas assumindo a distribuição ACBVE (2.1) são obtidas usando-se resultados assintóticos dos estimadores de máxima verossimilhança. Na prática, esses resultados podem não ser muito precisos mesmo para tamanhos amostrais razoavelmente grandes e dependem de uma boa parametrização (ver por exemplo, ACHCAR, 1995). 


\section{Capítulo 4}

\section{Alguns aspectos sobre a simula-}

ção de dados de tempo de vi-

\section{da bivariados com a distribui-}

\section{ção ACBVE}

Para gerar uma amostra aleatória de observações de tempos de vida bivariados $X$ e $Y$ segundo a distribuição Acbve (2.1) de Block \& Basu de tamanho $n$, seguiram-se os seguintes passos:

1. Gerou-se um número $N(N>n)$ de observações $W$, onde $W=\min (X, Y)$ tem uma distribuição exponencial com densidade $f(w)=\lambda e^{-\lambda w}$ com $\lambda=\lambda_{1}+\lambda_{2}+\lambda_{3}$

2. Associado a cada valor de $W$, considerou-se uma variável indicadora $\delta$, onde $\delta=1$ se $X<Y$ e $\delta=0$ se $X \geq Y$. Assim, geraram-se $N$ observações $\delta_{i}$ da distribuição Bernoulli com parâmetro $p$ dado por 


$$
p=P(X<Y)=\int_{0}^{\infty} \int_{x}^{\infty} \frac{\lambda \lambda_{1} \lambda_{23}}{\lambda_{12}} e^{-\lambda_{1} x-\lambda_{23} y} d y d x
$$

Isto é,

$$
p=\frac{\lambda_{1}}{\lambda_{12}}
$$

3. Dos passos (1) e (2), tem-se uma amostra de tamanho $N$ de pares $\left(W_{i}, \delta_{i}\right)$, $i=1,2,3, \ldots, N$, onde $\delta_{i}=1$ ou 0 . Se $\delta_{i}=1$, isto é, $X_{i}<Y_{i}$, tem-se $W_{i}=X_{i}$ e pode-se prever um valor para $Y_{i}$ e assim ter o tempo de vida bivariado $\left(X_{i}, Y_{i}\right)$. Se $\delta_{i}=0$, isto é, $X_{i} \geq Y_{i}$, tem-se $W_{i}=Y_{i}$ e pode-se prever um valor para $X_{i}$ e assim ter o tempo de vida bivariado $\left(X_{i}, Y_{i}\right)$.

4. Com os dados gerados obtidos $\left(X_{i}, Y_{i}\right), i=1,2, \ldots, N$ seguindo os passos (1), (2) e (3), constrói-se uma tabela de distribuição de frequência observada com $K_{1} \times K_{2}$ classes da forma $\left(a_{1 j}<X<b_{1 j}, a_{2 l}<Y<b_{2 l}\right)$, $j=1,2, \ldots, K_{1}$ e $l=1,2, \ldots, K_{2}$.

5. Para obter uma amostra aleatória de tamanho $n(n<N)$, escolheram-se arbitrariamente $n p_{j l}$ observações para cada classe de frequência

$$
\left(a_{1 j}<X<b_{1 j}, a_{2 l}<Y<b_{2 l}\right)
$$

onde

$$
p_{j l}=P\left\{a_{1 i j}<X<b_{1 j}, a_{2 l}<Y<b_{2 l}\right\}
$$

sendo que $(X, Y)$ tem distribuição ACBve (2.1).

Isto é, 


$$
p_{j l}= \begin{cases}\phi_{1}\left(\lambda_{1}, \lambda_{2}, \lambda_{3}\right) & \text { se } b_{1 j} \leq a_{2 l} \\ \phi_{2}\left(\lambda_{1}, \lambda_{2}, \lambda_{3}\right) & \text { se } b_{2 l}<a_{1 j} \\ \phi_{2}\left(\lambda_{1}, \lambda_{2}, \lambda_{3}\right) & \text { se } a_{1 j}=a_{2 l} \text { e } b_{1 j}=b_{2 l}\end{cases}
$$

onde

$$
\begin{aligned}
\phi_{1}\left(\lambda_{1}, \lambda_{2}, \lambda_{3}\right)= & \frac{\lambda}{\lambda_{12}}\left(e^{-\lambda_{1} b_{1 j}}-e^{-\lambda_{1} a_{1 j}}\right)\left(e^{-\lambda_{23} b_{2 l}}-e^{-\lambda_{23} a_{2 l}}\right) \\
\phi_{2}\left(\lambda_{1}, \lambda_{2}, \lambda_{3}\right)= & \frac{\lambda}{\lambda_{12}}\left(e^{-\lambda_{2} a_{2 l}}-e^{-\lambda_{2} b_{2 l}}\right)\left(e^{-\lambda_{13} a_{1 j}}-e^{-\lambda_{13} b_{1 j}}\right) \\
\phi_{3}\left(\lambda_{1}, \lambda_{2}, \lambda_{3}\right)= & \frac{\lambda}{\lambda_{12}} e^{-\lambda_{23} b_{2 l}}\left(e^{-\lambda_{1} b_{1 j}}-e^{-\lambda_{1} a_{1 j}}\right)+ \\
& \frac{\lambda}{\lambda_{12}} e^{-\lambda_{2} a_{2 l}}\left(e^{-\lambda_{13} a_{1 j}}-e^{-\lambda_{13} b_{1 j}}\right)+ \\
& \frac{\lambda_{3}}{\lambda_{12}}\left(e^{-\lambda b_{1 j}}-e^{-\lambda a_{1 j}}\right)
\end{aligned}
$$

\subsection{Predicão para $Y$}

Quando $\delta=1$, isto é, $W=X$, um valor predito para $Y$ (ver por exemplo, BicKel \& DoKsum, 1977) é dado por:

$$
E(Y \mid Y>x)=\int_{x}^{\infty} \frac{y f_{Y}(y) d y}{P(Y>x)}
$$

onde $f_{Y}(y)$ é a função densidade de probabilidade marginal para $Y$ dada por (2.8).

$$
\text { Isto é, }
$$

$$
E(Y \mid Y>x)=\frac{\lambda}{\lambda_{12} P(Y>x)}\left\{x e^{-\lambda_{23} x}+\frac{1}{\lambda_{23}} e^{-\lambda_{23} x}-\frac{\lambda_{3}}{\lambda} x e^{-\lambda x}-\frac{\lambda_{3}}{\lambda^{2}} e^{-\lambda x}\right\}
$$

onde

$$
P(Y>x)=\frac{\lambda}{\lambda_{12}} e^{-\lambda_{23} x}-\frac{\lambda_{3}}{\lambda_{12}} e^{-\lambda x}
$$




\subsection{Predicão para $X$}

Quando $\delta=0$, isto é, $W=Y$, um valor predito para $\mathrm{X}$ é dado por

$$
E(X \mid X>y)=\int_{y}^{\infty} \frac{x f_{X}(x) d x}{P(X>y)}
$$

onde $f_{X}(x)$ é a função densidade de probabilidade marginal para $X$ segundo (2.8 ).

$$
E(X \mid X>y)=\frac{\lambda}{\lambda_{12} P(X>y)}\left\{y e^{-\lambda_{13} y}+\frac{1}{\lambda_{13}} e^{-\lambda_{13} y}-\frac{\lambda_{3}}{\lambda} y e^{-\lambda y}-\frac{\lambda_{3}}{\lambda^{2}} e^{-\lambda_{y}}\right\}
$$

onde

$$
P(X>y)=\frac{\lambda}{\lambda_{12}} e^{-\lambda_{13} y}-\frac{\lambda_{3}}{\lambda_{12}} e^{-\lambda y}
$$

Usando este procedimento de simulação podem-se gerar dados de sobrevivência bivariados com a distribuição ACBVE (2.1) de BLOCK \& BASU. Um programa computacional para gerar esses dados foi desenvolvido usando o software SAS por LeAndro \& ACHCAR (1995). (Programa 1) 


\section{Capítulo 5}

\section{Aspectos de Reparametrização para a distribuição ACBVE}

É bem conhecido que a parametrização de um modelo estatístico afeta o desempenho das técnicas numéricas usadas para fazer inferências (no caso Bayesiano) e pode afetar a precisão de resultados assintóticos dos estimadores de máxima verossimilhança (no caso clássico), principalmente quando o tamanho da amostra é pequeno ou moderado. Quando se trabalha no contexto Bayesiano, métodos de integração são de suma importância, pois, usualmente, o estatístico Bayesiano tem interesse em achar momentos e densidades marginais a posteriori de interesse; as quais envolvem o cálculo de integrais. Ocorre que, frequentemente, estas integrais não têm forma analítica fechada e, portanto, existe a necessidade de utilizar métodos númericos, tais como: método de integração numérica de Simpson ou quadratura gaussiana (ver por exemplo, NAYLOR \& SMITH, 1982) e outros, ou métodos de aproximações tais como de Tierney \& Kadane (1986) ou ainda, procedimentos de Monte Carlo ou de "Gibbs Sampler" (ou amostrador de Gibbs) (ver por exemplo, Gelfand \& SMITH, 1990) para sua resolução . Entretanto, a precisão e a eficiência destes métodos podem depender criticamente da parametrização adotada no modelo (ver por exemplo, ACHCAR \& SMITH, 1990). Em termos 
gerais, muitas técnicas númericas e de aproximações têm desempenho melhor quando for usada uma reparametrização que torna a distribuição a posteriori "mais próxima" da normal. Assim, um problema importante com que se deparam os estatísticos é o de achar uma transformação biunívoca de $\theta$ para $\phi$ tal que a função de verossimilhança ou a densidade a posteriori de $\phi$ é melhor comportada.

Existem muitas abordagens para determinar a proximidade da normalidade. Entretanto, muitos destes métodos não têm implementação operacional direta, assim não é fácil quantificar o quanto uma parametrização particular está distante da normal. Muitos produzem medidas complicadas intratáveis, difíceis de calcular ou de interpretar.

KASS \& SlATE (1992) propuseram uma medida para diagnosticar a não normalidade das densidades a posteriori e da função de verossimilhança. Neste trabalho utiliza-se esta medida para avaliar a normalidade conjunta da função de verossimilhança para os parâmetros $\lambda_{1}, \lambda_{2}$ e $\lambda_{3}$ e também em diferentes reparametrizações destes parâmetros. Uma dessas medidas para o caso multiparamétrico é dada por:

$$
S T D=\sum_{i j k l m n} b_{i l} b_{j m} b_{k n} d_{i j k} d_{l m n}
$$

onde, $b_{i j}$ são os elementos da inversa da matriz de informação observada, $d_{i j k}=$ $\frac{\partial^{3} l(\hat{\theta})}{\partial \theta_{i} \partial \theta_{j} \partial \overline{\theta_{k}}}$, para um parâmetro $m$-dimensional $\theta$ e a soma é sobre todos os índices. Pode-se, em alguns casos, verificar a normalidade da função de verossimilhança ou da densidade a posteriori com a ajuda de gráficos de contornos.

HiLls \& SMITH (1993) propõem uma técnica gráfica chamada "tplot", para verificar a "proximidade da normalidade" para a distribuição a posteriori . Este novo diagnóstico pode ser aplicado aos parâmetros de qualquer modelo estatístico e pode ser interpretado como uma medida da diferença entre a distribuição a posteriori e a aproximação normal para variação dos valores paramétricos. De maneira análoga, pode-se substituir a distribuição a posteriori pela função de 
verossimilhança. O uso recomendado do diagnóstico proposto por Hills \& SMITH é:

Plotar $T\left(\theta_{j}\right)$ calculado para diferentes valores de $\theta_{j}$ contra os valores $\theta_{j}$. O resultado gráfico será uma reta se o parâmetro estimado para $\theta_{j}$, unidimensional, for normalmente distribuído. $T\left(\theta_{j}\right)$ é dado por:

$$
T\left(\theta_{j}\right)=\operatorname{sgn}\left(\theta_{j}-\hat{\theta}_{j}\right)\left[-2 \ln \left\{\frac{p\left(\theta_{j} \mid x\right)}{p(\hat{\theta} \mid x)}\right\}\right]^{1 / 2}
$$

Apresentam-se a seguir alguns aspectos da reparametrização considerando-se o Modelo de BlOcK \& BASU (2.1).

Procurando por reparametrizações que melhoram a normalidade conjunta da função de verossimilhança foram consideradas diferentes reparametrizações para o modelo de ACBVE de BLOCK \& BASU e para avaliar a normalidade conjunta da função de verossimilhança na reparametrização considerada utiliza-se a medida de KASS \& SLATE, STD, dada pela expressão (5.1).

\subsection{Reparametrização Logaritmica}

Considerando-se a reparametrização logarítmica para os parâmetros $\lambda_{1}, \lambda_{2}, \lambda_{3}$ tem-se:

$$
\begin{aligned}
& \theta_{1}=\ln \lambda_{1}, \\
& \theta_{2}=\ln \lambda_{2}, \\
& \theta_{3}=\ln \lambda_{3},
\end{aligned}
$$

$\mathrm{E}$ assim a função de verossimilhança é dada por:

$$
\begin{aligned}
L\left(\theta_{1}, \theta_{2}, \theta_{3}\right)= & \frac{\theta_{1}^{r} \theta^{n} \theta_{2}^{n-r} \theta_{23}^{r} \theta_{13}^{n-r}}{\theta_{12}^{n}} \\
& \exp \left\{-\theta_{1} n \bar{x}-\theta_{2} n \bar{y}-\theta_{3} R m\right\}
\end{aligned}
$$


onde $\theta_{12}=\theta_{1}+\theta_{2}, \quad \theta_{13}=\theta_{1}+\theta_{3}, \quad \theta_{23}=\theta_{2}+\theta_{3}$ e $\theta=\theta_{1}+\theta_{2}+\theta_{3}$

Considerando-se que $\left(\hat{\theta_{1}}, \hat{\theta_{2}}, \hat{\theta_{3}}\right) \stackrel{a}{\sim} N_{3}\left[\left(\theta_{1}, \theta_{2}, \theta_{3}\right), \mathcal{I}^{-1}\right]$ onde $\mathcal{I}$ é a matriz de informação observada, podem-se calcular os intervalos de confiança para $\lambda_{1}, \lambda_{2}, \lambda_{3}$

\subsection{Transformação de Box \& Cox}

Considerando-se agora a família de transformações de Box \& Cox (1964) para reparametrizar $\left(\lambda_{1}, \lambda_{2}, \lambda_{3}\right)$ :

$$
\phi_{i}=\lambda_{i}^{\left(\xi_{i}\right)}=\left\{\begin{array}{cc}
\frac{\lambda_{i}^{\xi_{i}}-1}{\xi_{i}}, & \xi_{i} \neq 0 \\
\ln \lambda_{i} & \xi_{i}=0
\end{array}\right.
$$

onde, por exemplo, pode-se variar $\xi_{i}$ na escala

$$
\xi_{i}=\{-1.0,-0.8,-0.6 .-0.4,-0.2,0.0,0.2,0.4,0.6,0.8,1.0\}
$$

para $i=1,2,3$, a função de verossimilhança é dada por:

$$
\begin{aligned}
L\left(\phi_{1}, \phi_{2}, \phi_{3}\right)= & \frac{\psi_{1}^{r}\left(\psi_{1}+\psi_{2}+\psi_{3}\right)^{n} \psi_{2}^{n-r}\left(\psi_{2}+\psi_{3}\right)^{r}\left(\psi_{1}+\psi_{3}\right)^{n-r}}{\left(\psi_{1}+\psi 2\right)^{n}} \\
& \exp \left\{-\psi_{1} n \bar{x}-\psi_{2} n \bar{y}-\psi_{3} R m\right\}
\end{aligned}
$$

onde $\psi_{1}=\left(\phi_{1} \xi_{1}+1\right)^{\frac{1}{\xi_{1}}}, \quad \psi_{2}=\left(\phi_{2} \xi_{2}+1\right)^{\frac{1}{\xi_{2}}} \quad$ e $\quad \psi_{3}=\left(\phi_{3} \xi_{3}+1\right)^{\frac{1}{\xi_{3}}}$

Considerando-se as $11^{3}$ combinações de valores $\left(\xi_{1}, \xi_{2}, \xi_{3}\right)$ podem-se encontrar os valores de $\left(\xi_{1}, \xi_{2}, \xi_{3}\right)$ que minimizam o valor de $S T D$ e assim obter os intervalos de confiança para $\left(\lambda_{1}, \lambda_{2}, \lambda_{3}\right)$, a partir da distribuição assintótica,

$$
\left(\hat{\phi}_{1}, \hat{\phi}_{2}, \hat{\phi}_{3}\right) \stackrel{a}{\sim} N\left(\left(\phi_{1}, \phi_{2}, \phi_{3}\right), \mathcal{I}^{-1}\right)
$$




\subsection{Reparametrização $\left(\lambda_{1}, \lambda_{2}, \theta_{0}\right)$}

Considerando-se o modelo (2.1) tem-se que a função de confiabilidade para um sistema em série no tempo $t_{0}$ é dada por:

$$
\begin{aligned}
& \theta_{0}=R_{S}\left(t_{0}\right)=P\left(T>t_{0}\right)=P\left(\min (X, Y)>t_{0}\right)=\exp \left\{-\lambda t_{0}\right\} \\
& \text { Assim, fazendo-se: } \\
& \lambda=\frac{-\ln \theta_{0}}{t_{0}} \\
& \lambda_{3}=\frac{-\ln \theta_{0}}{t_{0}}-\lambda_{1}-\lambda_{2}, \\
& \lambda_{23}=\frac{-\ln \theta_{0}}{t_{0}}-\lambda_{1} \\
& \lambda_{13}=\frac{-\ln \theta_{0}}{t_{0}}-\lambda_{2}
\end{aligned}
$$

Pode-se, assim, expressar (3.1) utilizando-se a reparametrização $\left(\lambda_{1}, \lambda_{2}, \theta_{0}\right)$ :

$$
\begin{gathered}
L\left(\lambda_{1}, \lambda_{2}, \theta_{0}\right)=\frac{\lambda_{1}^{r} \lambda_{2}^{n-r}}{\left(\lambda_{1}+\lambda_{2}\right)^{n}}\left(\frac{-\ln \theta_{0}}{t_{0}}\right)^{n}\left(\frac{-\ln \theta_{0}}{t_{0}}-\lambda_{1}\right)^{r}\left(\frac{-\ln \theta_{0}}{t_{0}}-\lambda_{2}\right)^{n-r} \\
\exp \left\{-\lambda_{1} n \bar{x}-\lambda_{2} n \bar{y}-\left(\frac{-\ln \theta_{0}}{t_{0}}-\lambda_{1}-\lambda_{2}\right) R m\right\}
\end{gathered}
$$

onde $0<\theta_{0} \leq 1$.

Inferências sobre $\lambda_{1}, \lambda_{2}$ e $\theta$ usualmente são obtidas utilizando a normalidade assintótica dos estimadores de máxima verossimilhança.

$$
\left(\hat{\lambda_{1}}, \hat{\lambda_{2}}, \hat{\theta_{0}}\right) \stackrel{a}{\sim} N\left(\left(\lambda_{1}, \lambda_{2}, \theta_{0}\right), \mathcal{I}^{-1}\right)
$$

onde $\mathcal{I}$ é a matriz de informação observada. 


\subsection{Reparametrização $\left(\lambda_{1}, \lambda_{2}, \phi_{G J}\right)$}

Procurando por transformações que melhorem a normalidade conjunta da verossimilhança (3.1) pode-se considerar a reparametrização proposta por GUERRERO \& JOHNSON (1982) dada por:

$$
\phi_{G J}(\xi)= \begin{cases}\frac{\left\{\left(\frac{\theta_{0}}{1-\theta_{0}}\right)^{\xi}-1\right\}}{\xi} & \text { se } \xi \neq 0 \\ \ln \theta & \text { se } \xi=0\end{cases}
$$

A transformação (5.7) tem inversa dada por:

$$
\theta_{0}= \begin{cases}\frac{(\phi \xi+1)^{1 / \xi}}{1+(\phi \xi+1)^{1 / \xi}} & \text { se } \xi \neq 0 \\ \exp \phi & \text { se } \xi=0\end{cases}
$$

A partir daqui utiliza-se $\phi_{G J}=\phi$ para simplificar a notação.

Assumindo o modelo (2.1), a função de verossimilhança na reparametrização $\left(\lambda_{1}, \lambda_{2}, \phi\right)$ é dada por:

1. Se $\xi=0$

$$
\begin{aligned}
L\left(\lambda_{1}, \lambda_{2}, \phi\right)= & \frac{\lambda_{1}^{r} \lambda_{2}^{n-r}}{\left(\lambda_{1}+\lambda_{2}\right)} w^{n}\left(w-\lambda_{1}\right)^{r}\left(w-\lambda_{2}\right)^{n-r} \\
& \exp \left\{-\lambda_{1} n \bar{x}-\lambda_{2} n \bar{y}-\left(w-\lambda_{1}-\lambda_{2}\right) R m\right\}
\end{aligned}
$$

onde $w=\frac{-\ln \theta_{0}}{t_{0}}$

2. Se $\xi \neq 0$

$$
L\left(\lambda_{1}, \lambda_{2}, \phi\right)=
$$




$$
\begin{gathered}
\frac{1}{\left(\lambda_{1}+\lambda_{2}\right)^{n}} \lambda_{1}^{r}(-p 1)^{n} \lambda_{2}^{n-r}\left(-p 1-\lambda_{1}\right)^{r}\left(-p 1-\lambda_{2}\right)^{n-r} \\
\exp \left\{-\lambda_{1} n \bar{x}-\lambda_{2} n \bar{y}-\left(-p 1-\lambda_{1}-\lambda_{2}\right) R m\right\}
\end{gathered}
$$

onde

$$
p 1=\frac{\ln \frac{(\xi \phi+1)^{1 / \xi}}{\left.(\xi \phi+1)^{1 / \xi}+1\right)}}{t_{0}}
$$

Calculando-se para cada tempo especificado $t_{0}$ a medida de KASS \&

SLATE (5.1), STD, para diferentes valores de $\xi$ encontra-se o valor de $\xi$ que minimiza STD. Considerando-se o valor de $\xi$ encontrado em cada tempo $t_{0}$ pode-se construir a função de verossimilhança e utilizar resultados assintóticos para fazer as inferências desejadas visto que:

$$
\left(\hat{\lambda_{1}}, \hat{\lambda_{2}}, \hat{\phi}\right) \stackrel{a}{\sim} N_{3}\left[\left(\lambda_{1}, \lambda_{2}, \phi\right) ; \mathcal{I}^{-1}\right)
$$

\subsection{Reparametrização natural}

Muitas vezes ocorre na prática que um especialista tem informações sobre os prováveis valores do tempo médio de vida do componente $1\left(\mu_{1}\right)$, do componente $2\left(\mu_{2}\right)$ e sobre a confiabilidade do sistema $\left(\theta_{0}\right)$ devido a sua experiência prática. Portanto, seria muito interessante utilizar esta informação. Isto pode ser implementado se for utilizada uma reparametrização que possa expressar $\lambda_{1}, \lambda_{2}, \lambda_{3}$ como função de $\mu_{1}, \mu_{2}, \theta_{0}$.

Considerando-se que

$$
\left\{\begin{array}{l}
\mu_{1}=\frac{1}{\lambda_{13}}+\frac{\lambda_{2} \lambda_{3}}{\lambda_{12} \lambda_{13} \lambda} \\
\mu_{2}=\frac{1}{\lambda_{23}}+\frac{\lambda_{1} \lambda_{3}}{\lambda_{12} \lambda_{23} \lambda} \\
\theta_{0}=\exp \left\{\lambda t_{0}\right\}
\end{array}\right.
$$


Pode-se obter através de cálculos simples

$$
\left\{\begin{array}{l}
\lambda_{1}=\psi_{1}\left(\mu_{1}, \mu_{2}, \theta_{0}\right) \\
\lambda_{2}=\psi_{2}\left(\mu_{1}, \mu_{2}, \theta_{0}\right) \\
\lambda_{3}=\frac{-\ln \theta_{0}}{t_{0}}-\psi_{1}\left(\mu_{1}, \mu_{2}, \theta_{0}\right)-\psi_{2}\left(\mu_{1}, \mu_{2}, \theta_{0}\right)
\end{array}\right.
$$

onde

$$
\begin{aligned}
& \lambda_{2}=\psi_{2}\left(\mu_{1}, \mu_{2}, \theta_{0}\right)=k_{0}-\left\{\frac{\left(k_{0} b_{1}-2 a_{1} a_{2}+\sqrt{k_{0}^{2} b_{1}^{2}+4 a_{1}^{2} a_{2}^{2}}\right)}{2 a_{1} b_{1}}\right\} \\
& \lambda_{1}=\psi_{1}\left(\mu_{1}, \mu_{2}, \theta_{0}\right)=\lambda_{2}\left\{\frac{k_{0}}{\left(k_{0}-\lambda_{2}\right) a_{1}}-1\right\}
\end{aligned}
$$

e $k_{0}=\frac{-\ln \theta_{0}}{t_{0}}, \quad a_{1}=\mu_{1} k_{0}-1, \quad a_{2}=\mu_{2} k_{0}-1 \quad$ e $\quad b_{1}=\mu_{1}-\mu_{2}$.

Nesta reparametrização temos que a função de verossimilhança é dada por:

$$
\begin{aligned}
L\left(\mu_{1}, \mu_{2}, \theta_{0}\right)= & \left(\phi_{6} \phi_{5}\right)^{r}\left\{\frac{-\ln \left(\theta_{0}\right)}{t_{0}}\right\}^{n} \phi_{6}^{n-r}\left(-\frac{\ln \left(\theta_{0}\right)}{t_{0}}-\phi_{6} \phi_{5}\right)^{r}\left(\frac{\phi_{4}}{2}\right)^{n-r} \\
& \frac{\exp \left(-\phi_{6} \phi_{5} n \bar{x}-\phi_{6} n \bar{y}-\left(-\phi_{6} \phi_{5}+1 / 2 \phi_{4}\right) R m\right)}{\left(\phi_{6} \phi_{5}-\frac{\ln \left(\theta_{0}\right)}{t_{0}}-\frac{\phi_{4}}{2}\right)^{n}}
\end{aligned}
$$

onde 


$$
\begin{aligned}
& \phi_{1}=-\frac{\mu_{1} \ln \left(\theta_{0}\right)}{t_{0}}-1 \\
& \phi_{2}=-\frac{\mu_{2} \ln \left(\theta_{0}\right)}{t_{0}}-1 \\
& \phi_{3}=-\frac{\ln \left(\theta_{0}\right)\left(\mu_{1}-\mu_{2}\right)}{t_{0}}-2 \phi_{1} \phi_{2}+\sqrt{\frac{\ln \left(\theta_{0}\right)^{2}\left(\mu_{1}-\mu_{2}\right)^{2}}{t_{0}^{2}}+4 \phi_{1}^{2} \phi_{2}^{2}} \\
& \phi_{4}=\frac{\phi_{3}}{\phi_{1}\left(\mu_{1}-\mu_{2}\right)} \\
& \phi_{5}=-2 \frac{\ln \left(\theta_{0}\right)\left(\mu_{1}-\mu_{2}\right)}{t_{0} \phi_{3}}-1 \\
& \phi_{6}=-\frac{\ln \left(\theta_{0}\right)}{\theta_{0}}-\frac{\phi_{4}}{2}
\end{aligned}
$$

\subsection{Reparametrização $\left(\mu_{1}, \mu_{2}, \phi_{G J}\right)$}

Procurando por reparametrizações que melhorem a normalidade conjunta da função de verossimilhança na parametrização $\mu_{1}, \mu_{2}, \theta_{0}$ considera-se a reparametrização de GuERRERo \& JoHNSON para $\theta_{0}=R\left(t_{0}\right)$ e assim a função de verossimilhança será expressa por:

$$
\begin{aligned}
L\left(\mu_{1}, \mu_{2}, \phi\right)= & \frac{\eta_{5}^{r}\left(-{\frac{\eta_{1}}{t_{0}}}^{n}\right)\left(-\frac{\eta_{1}}{t_{0}}-\frac{\eta_{3}}{2}\right)^{n-r}\left(-\frac{\eta_{1}}{t_{0}}-\eta_{5}\right)^{r}\left(\frac{\eta_{3}}{2}\right)^{n-r}}{\left(\eta_{5}-\frac{\eta_{1}}{t_{0}}-\frac{\eta_{3}}{2}\right)^{n}} \\
& \exp \left\{-\left(-\frac{\eta_{1}}{t_{0}}-1 / 2 \eta_{3}\right) \eta_{4} n \bar{x}-\left(-\frac{\eta_{1}}{t_{0}}-1 / 2 \eta_{3}\right) n \bar{y}\right\} \\
& \exp \left\{-\left(-\eta_{5}+1 / 2 \eta_{3}\right) R m\right\}
\end{aligned}
$$


onde

$$
\begin{aligned}
\eta_{1}= & \ln \frac{(\xi \phi+1)^{1 / \xi}}{(\xi \phi+1)^{1 / \xi}+1} \\
\eta_{2}= & -\frac{\eta_{1}\left(\mu_{1}-\mu_{2}\right)}{t_{0}}-2\left(\frac{-\mu_{1} \eta_{1}}{t_{0}}-1\right)\left(\frac{-\mu_{2} \eta_{1}}{t_{0}}-1\right) \\
& +\sqrt{-\frac{\eta_{1}^{2}\left(\mu_{1}-\mu_{2}\right)^{2}}{t_{0}^{2}}+4\left(\frac{-\mu_{1} \eta_{1}}{t_{0}}-1\right)^{2}\left(\frac{-\mu_{2} \eta_{1}}{t_{0}}-1\right)^{2}} \\
\eta_{3}= & \frac{\eta_{2}}{\left(-\frac{\mu_{1} \eta_{1}}{t_{0}}-1\right)\left(\mu_{1}-\mu_{2}\right)} \\
\eta_{4}= & -2 \frac{\eta_{1}\left(\mu_{1}-\mu_{2}\right)}{t_{0} \eta_{2}}-1 \\
\eta_{5}= & \left(\frac{-\eta_{1}}{t_{0}}-\frac{1}{2} \eta_{3}\right) \eta_{4}
\end{aligned}
$$

Com essas diferentes parametrizações podem-se obter resultados inferenciais distintos baseados na teoria assintótica dos estimadores de máxima verossimilhança especialmente para amostras pequenas ou moderadas. É necessário em cada aplicação fazer uma análise exploratória das diferentes parametrizações para se obterem resultados mais precisos. Para isso utiliza-se a medida proposta por Kass \& Slate $(1992,1994)$ dada por (5.1) e/ou o "t-plot" proposto Hills \& SмITH (1993) dado por (5.2). 


\section{Capítulo 6}

\section{Análise Bayesiana do Modelo de}

\section{Block \& Basu}

No capítulo 3 foi considerada uma análise clássica do modelo ACBVE de BLOCK \& BASU baseada em resultados assintóticos que são válidos para amostras de tamanho grande. Para amostras de tamanhos moderados ou pequenos esses resultados assintóticos podem ser pobres. Como uma alternativa considerouse a utilização de reparametrizações que melhoram a "proximidade" à distribuição normal, no capítulo 5. Neste capítulo apresenta-se uma análise considerando-se a abordagem Bayesiana na qual informações referentes aos parâmetros são obtidas através de distribuições marginais a posteriori. Neste ponto surgem duas dificuldades, a saber:

i) A obtenção da distribuição a posteriori marginal.

ii) Cálculo dos momentos a posteriori de interesse.

Em ambos os casos é necessário a resolução de integrais que muitas vezes não apresentam solução analítica. Nesse caso, métodos de aproximação ou 
métodos numéricos são necessários. Um método que tem sido muito utilizado é o método de aproximação de Laplace. Ocorre que para sua aplicação é necessário encontrar um ponto de máximo de uma função que depende do parâmetro e assim à medida que a dimensão do espaço paramétrico aumenta, aumentam as dificuldades para sua obtenção e a aplicação do método vai se tornando mais trabalhosa e difícil de ser implementada. Para contornar esse problema apresenta-se neste trabalho a abordagem Bayesiana utilizando-se o amostrador de Gibbs associado ao algoritmo de Metropolis-Hastings (G-MH) .

\subsection{Análise Bayesiana I}

Nesta seção será considerada uma análise Bayesiana utilizando-se o método de aproximação de Laplace introduzido por Tierney \& Kadane (1986). $\mathrm{Na}$ abordagem Bayesiana as densidades marginais e os momentos a posteriori são obtidos através de integração. Assim, por exemplo, a densidade a posteriori marginal para $\lambda_{3}$, considerando-se a priori não-informativa de Jeffreys (ver por exemplo, Box \& TIAO, 1973) dada por,

$$
\pi\left(\lambda_{1}, \lambda_{2}, \lambda_{3}\right) \propto\left[\operatorname{det} I\left(\lambda_{1}, \lambda_{2}, \lambda_{3}\right)\right]^{1 / 2}
$$

onde $I\left(\lambda_{1}, \lambda_{2}, \lambda_{3}\right)$ é a matriz de informação de Fisher para $\left(\lambda_{1}, \lambda_{2}, \lambda_{3}\right)$, é obtida integrando a densidade a posteriori conjunta para $\left(\lambda_{1}, \lambda_{2}, \lambda_{3}\right), \pi\left(\lambda_{1}, \lambda_{2}, \lambda_{3} \mid \mathcal{D}\right)$, com relação aos parâmetros $\lambda_{1}$ e $\lambda_{2}$ isto é:

$$
\pi\left(\lambda_{3} \mid \mathcal{D}\right)=\int_{0}^{\infty} \int_{0}^{\infty} \pi\left(\lambda_{1}, \lambda_{2}, \lambda_{3} \mid \mathcal{D}\right) d \lambda_{1} d \lambda_{2}
$$

Como a integral (6.2) não possui solução analítica explícita é necessário utilizar métodos numéricos ou métodos de aproximação para obter uma 
solução aproximada. Utilizando-se o método de aproximação de Laplace uma aproximação é dada por,

$$
\pi\left(\lambda_{3} \mid \mathcal{D}\right) \propto\left\{\operatorname{det}\left[n D^{2} h_{\lambda_{3}}\left(\tilde{\lambda}_{1}, \tilde{\lambda}_{2}\right)\right]\right\}^{-1 / 2} f_{\lambda_{3}}\left(\tilde{\lambda}_{1}, \tilde{\lambda}_{2}\right) e^{-n h_{\lambda_{3}}\left(\tilde{\lambda}_{1}, \tilde{\lambda}_{2}\right)}
$$

onde

$$
\begin{gathered}
f_{\lambda_{3}}\left(\lambda_{1}, \lambda_{2}\right)=\left[\operatorname{det} I\left(\lambda_{1}, \lambda_{2}, \lambda_{3}\right)\right]^{1 / 2}, \\
-n h_{\lambda_{3}}\left(\lambda_{1}, \lambda_{2}\right)=r \ln \left(\lambda_{1}\right)+n \ln (\lambda)+(n-r) \ln \left(\lambda_{2}\right)+r \ln \left(\lambda_{23}\right)+ \\
(n-r) \ln \left(\lambda_{13}\right)-n \ln \left(\lambda_{12}\right)-\lambda_{1} n \bar{x}-\lambda_{2} n \bar{y}-\lambda_{3} R m,
\end{gathered}
$$

$\left(\tilde{\lambda}_{1}, \tilde{\lambda}_{2}\right)$ são os valores de $\lambda_{1}$ e $\lambda_{2}$ que maximizam a função $-n h_{\lambda_{3}}\left(\lambda_{1}, \lambda_{2}\right)$ para cada $\lambda_{3}$, ou seja, são a solução do sistema de equações simultâneas (6.5):

$$
\left\{\begin{array}{l}
\frac{\partial}{\partial \lambda_{1}}\left(-n h_{\lambda_{3}}\left(\lambda_{1}, \lambda_{2}\right)\right)=\frac{r}{\lambda_{1}}+\frac{n}{\lambda_{123}}+\frac{n-r}{\lambda_{13}}-\frac{n}{\lambda_{12}}-n \bar{x}=0 \\
\frac{\partial}{\partial \lambda_{2}}\left(-n h_{\lambda_{3}}\left(\lambda_{1}, \lambda_{2}\right)\right)=\frac{n}{\lambda_{123}}+\frac{n-r}{\lambda_{2}}+\frac{r}{\lambda_{23}}-\frac{n}{\lambda_{12}}-n \bar{y}=0
\end{array}\right.
$$

e $n D^{2} h_{\lambda_{3}}\left(\tilde{\lambda}_{1}, \tilde{\lambda}_{2}\right)$ é a matriz Hessiana de $n h_{\lambda_{3}}\left(\lambda_{1}, \lambda_{2}\right)$ avaliada em $\left(\tilde{\lambda}_{1}, \tilde{\lambda}_{2}\right)$. Devese observar que o sistema (6.5) depende de $\lambda_{3}$. Assim, só se podem obter valores aproximados pontuais para a densidade a posteriori marginal, isto é, fixando-se $\lambda_{3}$ obtém-se um valor aproximado pontual para a densidade a posteriori marginal. Ainda, algumas vezes utiliza-se uma priori não-informativa mais por conveniência matemática do que devido a argumentos motivados realisticamente e assim a incorporação de uma densidade informativa pode complicar ainda mais a obtenção da solução aproximada. 
De forma similar, podem-se obter as densidades a posteriori marginais aproximadas pelo método de Laplace para os parâmetros $\lambda_{1}$ e $\lambda_{2}$ e para momentos a posteriori de interesse, como funções $g\left(\lambda_{1}, \lambda_{2}, \lambda_{3}\right)$.

É importante salientar que se podem considerar outras escolhas para a função $f_{\lambda_{3}}\left(\lambda_{1}, \lambda_{2}\right)$ em (6.3). Um caso especial é dado por $f_{\lambda_{3}}()=$.1 (ver por exemplo, Tierney \& Kadane, 1986).

Neste trabalho devido às dificuldades apresentadas pelo método de Laplace, adota-se outra metodologia para obtenção das densidades marginais. Esta metodologia utiliza simulação iterativa, isto é, obtém-se uma amostra da distribuição a posteriori de interesse através da amostragem de Gibbs associado ao algoritmo de Metropolis-Hastings. A simulação iterativa facilita bastante a obtenção das estat'isticas a posteriori de interesse, pois através da amostra da distribuição $a$ posteriori marginal obtida calculam-se os momentos de interesse, de forma extremamente fácil utilizando estatísticas descritivas e o método de estimação de Monte Carlo.

\subsection{Análise Bayesiana II}

Para obtenção das estatísticas a posteriori marginais de interesse utiliza-se o algoritmo do amostrador de Gibbs que é uma técnica para gerar variáveis aleatórias de uma distribuição marginal indiretamente, isto é, este método permite gerar variáveis aleatórias de uma distribuição marginal sem ter que calcular a sua densidade. Assim, através da simulação de uma amostra suficientemente grande pode-se calcular a média, a variância e outras características de interesse desta distribuição.

Em síntese, o esquema de atualização markoviano Gibbs é definido por: dado um conjunto arbitrário inicial de valores $\boldsymbol{\theta}^{0}=\left(\theta_{1}^{0}, \theta_{2}^{0}, \cdots, \theta_{k}^{0}\right)$ e a função de densidade conjunta a posteriori $\pi(\theta \mid \mathcal{D})$ pode-se proceder à atualização 
de $\theta^{0}$ via um ciclo de "visitas" à cada uma das funções de distribuições a posteriori condicionais cheias (ver por exemplo, GELFAND \& SMITH, 1990). Desta forma cada variável é visitada em sua ordem natural e assim que o ciclo se completar um novo valor para $\boldsymbol{\theta}^{\mathbf{0}}$ será obtido, $\boldsymbol{\theta}^{\mathbf{1}}$. Após $t$ repetições deste ciclo chega-se a $\boldsymbol{\theta}^{t}=\left(\theta_{1}^{t}, \cdots, \theta_{k}^{t}\right)$. Sob algumas condições de regularidade suaves, GEMAN \& GEMAN (1984) mostram que o ponto $k$-dimensional $\left\{\theta_{1}^{(t)}, \theta_{2}^{(t)}, \cdots, \theta_{k}^{(t)}\right\}$ produzido na t-ésima iteração do esquema amostral converge em distribuição para uma variável aleatória da distribuição $p\left(\theta_{1}, \theta_{2}, \cdots, \theta_{k} \mid \mathcal{D}\right)$ se $t$ for suficientemente grande. Além disso, $\theta_{j}^{k}$ pode ser considerado como uma observação simulada de $p\left(\theta_{j} \mid \mathcal{D}\right)$, a distribuição a posteriori marginal de $\theta_{j}$. Da convergência do amostrador de Gibbs, qualquer característica da densidade a posteriori marginal $p\left(\theta_{j} \mid \mathcal{D}\right)$ pode ser obtida. Na prática, entretanto, ainda existe muita discussão sobre o monitoramento da convergência.

Ocorre que muitas vezes não é tão fácil amostrar de funções de distribuições a posteriori condicionais cheias por esta possuir uma forma analítica muito complicada e diferente das formas padrões conhecidas ou facilmente amostradas. Assim pode ser problemática a atualização de $\theta_{j}^{i}$ para algum $j=1, \cdots, k, \forall i$. Logo, para continuação do ciclo (ou do processo de atualização) é necessário empregar mecanismos que tornem possível a obtenção deste novo valor. Neste momento, surge então a necessidade da utilização, por exemplo, do algoritmo de MetropolisHastings (ver por exemplo, Chib \& Greenberg, 1995). Para aplicação do algoritmo de Metropolis-Hastings é necessário encontrar uma função de distribuição denominada núcleo de transição o qual será responsável pela geração de uma possível atualização para $\theta_{j}^{i}$ em cada iteração $i$ para algum $j$. A decisão da atualização de $\theta_{j}^{i}$ será feita via uma distribuição de Bernoulli com parâmetro $p$, (probabilidade de transição). Nos problemas apresentados neste trabalho é explorada a forma da distribuição em questão para obtenção do núcleo de transição. Ocorrem, frequentemente, neste processo de atualização muitas rejeições do novo valor gerado e uma forma para contornar esse problema é através da introdução de uma variável laten- 
te ao sistema inferencial (uma variável que não é observada mas que simplifica o processo inferencial) (ver por exemplo, TANNER \& WONG, 1987). Neste contexto a introdução da variável latente diminuirá (não eliminará) o número de rejeições.

Neste trabalho considerando-se o algoritmo de Gibbs associado ao algoritmo de Metropolis-Hastings geram-se $m$ sequências de tamanho $2 p$ com valores iniciais distintos para cada uma delas, desprezam-se as $p$ primeiras iterações com a finalidade de diminuir o efeito da estimativa inicial em cada uma das sequências consideradas. Com as $p$-ésimas iterações restantes escolhe-se uma amostra de tamanho $2 n$ com espaçamento entre as iterações igual a $p / 2 n$.

Para monitorar a convergência da simulação iterativa utiliza-se a técnica proposta por Gelman \& Rubin (1992). Esta técnica utiliza o valor da variância entre a média das $m$ sequências e o valor da média das $m$ variâncias dentro de cada sequência para estimar um fator $\hat{R}$ pelo qual a escala da distribuição atual de cada parâmetro pode ser reduzido se o número de iterações tender ao infinito. Uma vez que $\hat{R}$ esteja próximo de 1 para todos os parâmetros pode-se considerar que a distribuição atual converge para a distribuição estacionária.

\subsubsection{Análise Bayesiana usando Gibbs com Metropolis- Hastings}

Assumindo-se dois tempos de falhas $X$ e $Y$ associados a cada unidade de observação com distribuição ACBVE de BLOCK \& BASU com função densidade conjunta (2.1), a função de verossimilhança é dada por

$$
L\left(\lambda_{1}, \lambda_{2}, \lambda_{3}\right)=\prod_{i=1}^{n} f_{1}^{\delta_{i}}\left(x_{i}, y_{i}\right) f_{2}^{1-\delta_{i}}\left(x_{i}, y_{i}\right)
$$

onde $\lambda_{12}=\lambda_{1}+\lambda_{2}, \quad \lambda_{13}=\lambda_{1}+\lambda_{3}, \quad \lambda_{23}=\lambda_{2}+\lambda_{3}, \quad \lambda=\lambda_{1}+\lambda_{2}+\lambda_{3}, \quad n \bar{x}=\sum_{i=1}^{n} x_{i}$, $n \bar{y}=\sum_{i=1}^{n} y_{i}, \quad r=\sum_{i=1}^{n} \delta_{i} \quad$ e $R m=\sum_{i=1}^{n}\left[\delta_{i} y_{i}+\left(1-\delta_{i}\right) x_{i}\right]$. 
Para obtenção das estatístícas a posteriori marginais de interesse utiliza-se o amostrador de Gibbs que é uma técnica para gerar variáveis aleatórias de uma distribuição marginal indiretamente, isto é, este método permite gerar variáveis aleatórias de uma distribuição marginal sem ter que calcular a sua densidade. Assim, através da simulação de uma amostra suficientemente grande podem-se calcular a média, a variância e outras características dessa distribuição. Portanto, baseado neste algoritmo pode-se obter uma amostra da densidade a posteriori conjunta (6.9).

Para inferência Bayesiana, considera-se a introdução de uma variável latente $N_{1}\left(N_{1}=\right.$ número de observações tais que $\left.X_{i}<Y_{i}\right)$, assumindo-se as seguintes densidades a priori para: $N_{1}, \lambda_{1}, \lambda_{2}$ e $\lambda_{3}$ :

$$
\begin{aligned}
& N_{1} \sim b\left(n, \frac{\lambda_{1}}{\lambda_{12}}\right) \\
& \lambda_{1} \sim \Gamma\left(a_{1}, b_{1}\right) \\
& \lambda_{2} \sim \Gamma\left(a_{2}, b_{2}\right) \\
& \lambda_{3} \sim \Gamma\left(a_{3}, b_{3}\right)
\end{aligned}
$$

onde $a_{i}$ e $b_{i}$ são conhecidos para $i=1,2,3$ obtidos através da interação entre pesquisador e estatístico, $b\left(n, \lambda_{1} / \lambda_{12}\right)$ denota uma distribuição binomial com probabilidade de sucesso $\lambda_{1} / \lambda_{12}, \Gamma\left(a_{i}, b_{i}\right)$ denota uma distribuição gama com média $a_{i} / b_{i} \mathrm{e}$ variância $a_{i} / b_{i}^{2}$. Assumindo-se independência a priori para $N_{1}, \lambda_{1}, \lambda_{2}$ e $\lambda_{3}$, a distribuição conjunta a posteriori para $N_{1}, \lambda_{1}, \lambda_{2}$ e $\lambda_{3}$ é dada por:

$$
\begin{aligned}
& \pi\left(N_{1}, \lambda_{1}, \lambda_{2}, \lambda_{3} \mid \mathcal{D}\right) \propto \\
& \quad\left(\begin{array}{c}
n \\
N_{1}
\end{array}\right)\left(\frac{\lambda_{1}}{\lambda_{12}}\right)^{N_{1}}\left(\frac{\lambda_{2}}{\lambda_{12}}\right)^{n-N_{1}} \frac{\lambda^{n}}{\lambda_{12}^{n}}
\end{aligned}
$$




$$
\begin{aligned}
& \lambda_{13}^{r} \lambda_{1}^{r+a_{1}-1} \lambda_{2}^{n-r+a_{2}-1} \lambda_{3}^{a_{3}-1} \lambda_{23}^{r} \\
& \exp \left\{-\left(n \bar{x}+b_{1}\right) \lambda_{1}-\left(n \bar{y}+b_{2}\right) \lambda_{2}-\left(R m+b_{3}\right) \lambda_{3}\right\}
\end{aligned}
$$

ou seja,

$$
\begin{aligned}
& \pi\left(N_{1}, \lambda_{1}, \lambda_{2}, \lambda_{3} \mid \mathcal{D}\right) \propto \\
& \quad\left(\begin{array}{c}
n \\
N_{1}
\end{array}\right)\left(\frac{\lambda^{n}}{\lambda_{12}^{2 n}}\right) \lambda_{23}^{r} \lambda_{13}^{n-r} \\
& \quad \lambda_{1}^{N_{1}+r+a_{1}-1} \lambda_{2}^{2 n-N_{1}-r+a_{2}-1} \lambda_{3}^{a_{3}-1} \\
& \quad \exp \left\{-\left(n \bar{x}+b_{1}\right) \lambda_{1}-\left(n \bar{y}+b_{2}\right) \lambda_{2}-\left(R m+b_{3}\right) \lambda_{3}\right\}
\end{aligned}
$$

onde $\mathcal{D}$ denota o conjunto de dados.

As densidades a posteriori condicionais cheias para aplicação do algoritmo de Gibbs são dadas por:

$$
\begin{aligned}
N_{1} \mid \lambda_{1}, \lambda_{2}, \lambda_{3}, \mathcal{D} & \sim b\left(n, \frac{\lambda_{1}}{\lambda_{12}}\right) \\
\pi\left(\lambda_{1} \mid N_{1}, \lambda_{2}, \lambda_{3}, \mathcal{D}\right) & \propto \frac{\lambda^{n}}{\lambda_{12}^{2 n}} \lambda_{13}^{n-r} \lambda_{1}^{N_{1}+r+a_{1}-1} \exp \left\{-\left(n \bar{x}+b_{1}\right) \lambda_{1}\right\} \\
\pi\left(\lambda_{2} \mid N_{1}, \lambda_{1}, \lambda_{3}, \mathcal{D}\right) & \propto \frac{\lambda^{n}}{\lambda_{12}^{2 n}} \lambda_{23}^{r} \lambda_{2}^{2 n-N 1-r+a_{2}-1} \exp \left\{-\left(n \bar{y}+b_{2}\right) \lambda_{2}\right\} \\
\pi\left(\lambda_{3} \mid N_{1}, \lambda_{1}, \lambda_{2}, \mathcal{D}\right) & \propto \lambda^{n} \lambda_{23}^{r} \lambda_{13}^{n-r} \lambda_{3}^{a_{3}-1} \exp \left\{-\left(R m+b_{3}\right) \lambda_{3}\right\}
\end{aligned}
$$

Considerando-se as distribuições condicionais para $\lambda_{1}, \lambda_{2}, \lambda_{3}$ dadas em (6.10) necessárias para aplicação da técnica do algoritmo Gibbs verifica-se 
que estas não possuem uma distribuição padrão, no sentido de que seja fácil gerar valores de variáveis que tenham essa distribuição; assim é necessário utilizar outra metodologia para obter os valores amostrais desejados. Para tanto utiliza-se o algoritmo de Metropolis- Hastings (ver por exemplo, Chib \& Greenberg, 1995) segundo o qual com a finalidade de gerar valores amostrais da distribuição de interesse é necessário explorar a forma conhecida da distribuição para especificar uma densidade geradora candidata (núcleo de transição). Por exemplo, se a distribuição condicional de interesse, $\pi(t)$ puder ser fatorada como $\pi(t)=\psi(t) h(t)$ onde $h(t)$ é uma distribuição que pode ser facilmente amostrada e se $\psi(t)$ for uniformemente limitada então poderemos amostrar $\pi(t)$ seguindo os seguintes passos:

1. Atribuir um valor inicial para $t$, dado por $t^{0}$.

2. Gerar um novo valor para $t, t^{G}$, via $h(t)$.

3. Considerar $p=\min \left\{\frac{\psi\left(t^{G}\right)}{\psi\left(t^{0}\right)}, 1\right\}$ (probabilidade de transição).

4. Gerar um valor $b$ de uma distribuição Bernoulli com probabilidade $p$ de sucesso.

5. Considerar um novo valor para $t, t^{1}=b t^{G}+(1-b) t^{0}$

6. Voltar ao passo 2 até a convergência ser atingida.

Assim o método de amostragem utilizado neste trabalho associa o amostrador de Gibbs com o método de "Metropolis-Hastings".

Para monitorar a convergência utilizou-se o método proposto por Gelman \& Rubin (1992), que utiliza a técnica da análise da variância para determinar a necessidade de um maior número de iterações. A técnica de Gelman \& RUBIN monitora a convergência da simulação iterativa calculando uma estimativa do fator $\hat{R}$ pelo qual a escala da distribuição estudada poderia ser reduzida se as simulações continuassem indefinidamente. Se o fator $\sqrt{\hat{R}}$ for alto deve-se considerar mais simulações para melhorar a inferência sobre a distribuição de interesse. Se $\hat{R} \approx 1$ a convergência ocorre e as amostras selecionadas das iterações são i.i.d.. 


\subsection{Estimadores de Bayes para os tempos de vi-}

\section{da médios}

Podem-se utilizar as amostras Gibbs para obter inferências para os parâmetros da distribuição ACBVE (2.1) ou funções desses parâmetros. Nesse caso, podem-se aproximar momentos a posteriori de interesse. Como um caso especial consideremos os tempos de vida médios,

$$
\begin{aligned}
& \mu_{1}=E[X]=\frac{\lambda_{12}+\lambda_{2} \lambda_{3}}{\lambda_{12} \lambda_{13} \lambda} \\
& \mu_{2}=E[Y]=\frac{\lambda \lambda_{12}+\lambda_{1} \lambda_{3}}{\lambda_{12} \lambda_{23} \lambda}
\end{aligned}
$$

Estimadores de Bayes para $\mu_{i}, i=1,2$ com relação à função de perda quadrática são dados por $E\left(\mu_{i} \mid \mathcal{D}\right)$ os quais podem ser aproximadados pela estimativa de Monte Carlo,

$$
\begin{aligned}
& \hat{\mu_{1}}=\frac{2}{R S} \sum_{s=1}^{S} \sum_{r=R / 2+1}^{R} \frac{\lambda^{(r, s)} \lambda_{12}^{(r, s)}+\lambda_{2}^{(r, s)} \lambda_{3}^{(r, s)}}{\lambda_{12}^{(r, s)} \lambda_{13}^{(r, s)} \lambda^{(r, s)}} \\
& \hat{\mu_{2}}=\frac{2}{R S} \sum_{s=1}^{S} \sum_{r=R / 2+1}^{R} \frac{\lambda^{(r, s)} \lambda_{12}^{(r, s)}+\lambda_{1}^{(r, s)} \lambda_{3}^{(r, s)}}{\lambda_{12}^{(r, s)} \lambda_{23}^{(r, s)} \lambda^{(r, s)}}
\end{aligned}
$$

onde $\lambda^{(r, s)}=\lambda_{1}^{(r, s)}+\lambda_{2}^{(r, s)}+\lambda_{3}^{(r, s)}, \lambda_{12}^{(r, s)}=\lambda_{1}^{(r, s)}+\lambda_{2}^{(r, s)}, \lambda_{13}^{(r, s)}=\lambda_{1}^{(r, s)}+\lambda_{3}^{(r, s)}$, $\lambda_{23}^{(r, s)}=\lambda_{2}^{(r, s)}+\lambda_{3}^{(r, s)}, \lambda_{1}^{(r, s)}, \lambda_{2}^{(r, s)}$ e $\lambda_{1}^{(r, s)}$ denotam os valores obtidos para $\lambda_{1}, \lambda_{2} \mathrm{e}$ $\lambda_{3}$ na $r$-ésima iteração e na $s$-ésima replicação onde $R$ e $S$ são respectivamente, o número total de iterações e replicações da amostra Gibbs. 


\subsection{Estimadores de Bayes para a função de con- fiabilidade}

Assumindo o modelo ACBVE (2.1), a função de confiabilidade para um sistema com dois componentes no tempo especificado $t_{0}$ é dado por,

$$
\begin{array}{ll}
R_{S}\left(t_{0}\right)=\exp \left\{-\lambda t_{0}\right\} & \text { para um sistema em série. } \\
R_{P}\left(t_{0}\right)=\frac{\left\{\lambda\left(e^{\lambda_{1} t_{0}}+e^{\lambda_{2} t_{0}}-1\right)-\lambda_{3}\right\}}{\lambda_{12} e^{\lambda t_{0}}} & \text { para um sistema em paralelo. }
\end{array}
$$

Estimadores de Bayes para $R_{S}\left(t_{0}\right)$ e $R_{P}\left(t_{0}\right)$ com relação a função de perda quadrática são dados por $E\left\{R_{S}\left(t_{0}\right) \mid \mathcal{D}\right\}$ e $E\left\{R_{P}\left(t_{0}\right) \mid \mathcal{D}\right\}$. Considerando-se a amostra de Gibbs, com $R$ iterações e $S$ replicações, as estimativas Monte Carlo para esses momentos a posteriori são dadas por

$$
\begin{aligned}
\hat{R}_{S}\left(t_{0}\right)= & \frac{2}{R S} \sum_{i=1}^{S} \sum_{r=R / 2+1}^{R} \exp \left\{-\lambda^{(r, s)} t_{0}\right\} \\
& \text { para um sistema em série. } \\
\hat{R_{P}}\left(t_{0}\right)= & \frac{2}{R S} \sum_{s=1}^{S} \sum_{r=R / 2+1}^{R} \frac{\left\{\lambda^{(r, s)}\left(e^{\lambda_{1}^{(r, s)} t_{0}}+e^{\lambda_{2}^{(r, s)} t_{0}}-1\right)-\lambda_{3}^{(r, s)}\right\}}{\lambda_{12}^{(r, s)} e^{\lambda^{(r, s)} t_{0}}}
\end{aligned}
$$

para um sistema em paralelo.

Também podem-se usar as amostras geradas de Gibbs para aproximar outros estimadores Bayesianos de interesse para a distribuição exponencial bivariada de Block \& BAsu. 


\section{Capítulo 7}

\section{Aplicação da distribuição}

\section{ACBVE em testes de vida acele-}

\section{rados}

Um dos grandes interesses da indústria é medir a confiabilidade dos seus produtos. Muitas vezes, isso é difícil porque experimentos conduzidos com esta finalidade são, em geral, caros e demandam muito tempo quando realizados sob condições normais de uso e assim, o risco do produto se tornar obsoleto é muito grande. Uma prática bastante utilizada nestes casos é aquela onde os produtos são submetidos a teste de vida acelerados, ou seja, com o objetivo de reduzir o tempo de vida submete-se cada unidade a provas em condições de funcionamento mais severas do que as normais ("Overstress"). Assim as unidades falham rapidamente reduzindo o tempo e o custo do experimento. "Overstress" envolve temperaturas altas, voltagem, pressão, vibração, carga etc., ou alguma combinação delas. A experiência dos engenheiros usualmente sugere as variáveis de estresse para um determinado produto ou material. A partir daí, resultados são extrapolados para produtos em condições normais de uso. 
Neste Capítulo considerando-se o modelo ACBVE (2.1) para dados de tempo de vida bivariados, a incorporação do fator estresse será feita utilizando-se o modelo de lei de potência (ver por exemplo, Mann, Schafer \& SingPurWALLA, 1974).

\subsection{O Modelo}

Considerando-se um sistema com dois componentes cujos tempos de vida são dados por $X$ e $Y, J$ níveis de estresse $V_{1}, V_{2}, \ldots, V_{J}$ e assumindo-se que testes de vida são conduzidos com a aplicação constante de um nf́vel de estresse selecionado obtém-se informação sobre os tempos de vida de cada componente sob o nível normal de estresse, $V_{0}$.

Considerando-se o modelo de potência (ver por exemplo, ManN, Schafer \& SingPuRWalla, 1974), dado por

$$
\lambda_{i j}=c_{i} V_{j}^{\mathcal{P}}
$$

onde $i=1,2,3, j=0,1,2, \ldots, J ; c_{1}, c_{2}, c_{3}$ e $\mathcal{P}$ são constantes e assumindo-se que $(X, Y)$ tem distribuição ACBVE (2.1) com parâmetros $\lambda_{10}, \lambda_{20}$ e $\lambda_{30}$ sob o nível de estresse normal $V_{0}$ e que sob um nível de estresse $V_{j}, j=1,2, \ldots, J,(X, Y)$ segue o modelo ACBVE (2.1) com parâmetros $\lambda_{1 j}, \lambda_{2 j} \mathrm{e} \lambda_{3 j}, j=1,2, \ldots, J$ e considerando-se $n_{j}$ unidades $\left(X_{1 j}, Y_{1 j}\right), \ldots,\left(X_{n_{j} j}, Y_{n_{j} j}\right)$ no começo de cada teste com estresse $V_{j}$, a função de verossimilhança para $c_{1}, c_{2}, c_{3}$ e $\mathcal{P}$ é dada por

$$
L_{j}\left(c_{1}, c_{2}, c_{3}, \mathcal{P}\right)=\prod_{i=1}^{n j} f_{1}^{\delta_{i j}}\left(X_{i j}, Y_{i j}\right) f_{2}^{1-\delta_{i j}}\left(X_{i j}, Y_{i j}\right)
$$

onde

$$
\delta_{i j}=1 \text { se } X_{i j}<Y_{i j} \text { e } \delta_{i j}=0 \text { se } X_{i j} \geq Y_{i j}
$$




$$
\begin{gathered}
f_{1}\left(X_{i j}, Y_{i j}\right)=\frac{c_{1} c_{23} c_{123}}{c_{12}} V_{j}^{2 \mathcal{P}} \exp \left\{-\left[c_{1} X_{i j}+c_{23} Y_{i j}\right] V_{j}^{\mathcal{P}}\right\} \\
f_{2}\left(X_{i j}, Y_{i j}\right)=\frac{c_{2} c_{13} c_{123}}{c_{12}} V_{j}^{2 \mathcal{P}} \exp \left\{-\left[c_{13} X_{i j}+c_{2} Y_{i j}\right] V_{j}^{\mathcal{P}}\right\} \\
c_{12}=c_{1}+c_{2} ; \quad c_{13}=c_{1}+c_{3} ; \quad c_{23}=c_{2}+c_{3} \text { e } c_{123}=c_{1}+c_{2}+c_{3}, \text { isto é } \\
L_{j}\left(c_{1}, c_{2}, c_{3}, \mathcal{P}\right)=\frac{c_{123}^{n_{j}} c_{1}^{r_{j}} c_{2}^{n_{j}-r_{j}} c_{23}^{r_{j}} c_{13}^{n_{j}-r_{j}}}{c_{12}^{n_{j}}}\left(V_{j}^{2 \mathcal{P}}\right)^{n_{j}} \\
\exp \left\{-\left[c_{1} n_{j} \bar{X}_{j}+c_{2} n_{j} \bar{Y}_{j}+c_{3} R_{j}\right] V_{j}^{\mathcal{P}}\right\}
\end{gathered}
$$

onde $\quad n_{j} \bar{X}_{j}=\sum_{i=1}^{n_{j}} X_{i j}, \quad n_{j} \bar{Y}_{j}=\sum_{i=1}^{n_{j}} Y_{i j}, \quad r_{j}=\sum_{i=1}^{n_{j}} \delta_{i j} \quad$ e $\quad R_{j}=\sum_{i=1}^{n_{j}}\left[\delta_{i j} Y_{i j}+\right.$ $\left.\left(1-\delta_{i j}\right) X_{i j}\right]$

Assumindo-se que os dados obtidos em cada nível de estresse $V_{1}, V_{2}, \ldots, V_{\mathrm{J}}$ são independentes, a função de verossimilhança para $c_{1}, c_{2}, c_{3}$ e $\mathcal{P}$ é dada por

$$
L\left(c_{1}, c_{2}, c_{3}, \mathcal{P}\right)=\prod_{j=1}^{J} L_{j}\left(c_{1}, c_{2}, c_{3}, \mathcal{P}\right)
$$

Isto é,

$$
\begin{aligned}
L\left(c_{1}, c_{2}, c_{3}, \mathcal{P}\right)= & \frac{c_{1}^{r} c_{23}^{r} c_{2}^{n-r} c_{13}^{n-r} c_{123}^{n}}{c_{12}^{n}} \prod_{j=1}^{J}\left(V_{j}^{2 \mathcal{P}}\right)^{n_{j}} \\
& \exp \left\{-\left[c_{1} S_{X}(\mathcal{P})+c_{2} S_{Y}(\mathcal{P})+c_{3} T(\mathcal{P})\right]\right\}
\end{aligned}
$$

onde $r=\sum_{j=1}^{J} r_{j}, \quad n=\sum_{j=1}^{J} n_{j}, \quad S_{X}(\mathcal{P})=\sum_{j=1}^{J} n_{j} \bar{X}_{j} V_{j}^{\mathcal{P}}, \quad S_{Y}(\mathcal{P})=\sum_{j=1}^{J} n_{j} \bar{Y}_{j} V_{j}^{\mathcal{P}}$ $T(\mathcal{P})=\sum_{j=1}^{J} R_{j} V_{j}^{\mathcal{P}}$

\subsection{Análise Clássica do Modelo}

\subsubsection{Estimadores de Máxima Verossimilhança}

Os estimadores de máxima verossimilhança para os parâmetros 
$c_{1}, c_{2}, c_{3}$ e $\mathcal{P}$ são os valores $\hat{c}_{1}, \hat{c}_{2}, \hat{c}_{3}$ e $\hat{\mathcal{P}}$ que maximizam a função de verossimilhança (7.5) ou equivalentemente, o logaritmo da função de verossimilhança dado por:

$$
l\left(c_{1}, c_{2}, c_{3}, \mathcal{P}\right)=\ln L\left(c_{1}, c_{2}, c_{3}, \mathcal{P}\right)
$$

ou seja,

$$
\begin{aligned}
l\left(c_{1}, c_{2}, c_{3}, \mathcal{P}\right)= & r \log c_{1}+(n-r) \log c_{2}+n \log c_{123}+r \log c_{23} \\
& +(n-r) \log c_{13}-n \log c_{12}+2 \mathcal{P} \sum_{i=1}^{J} n_{j} \log V_{j}- \\
& c_{1} S_{X}(\mathcal{P})-c_{2} S_{Y}(\mathcal{P})-c_{3} T(\mathcal{P})
\end{aligned}
$$

Considerando-se a equação (7.6) tem-se que as derivadas parciais de primeira ordem do logaritmo da função de verossimilhança para $c_{1}, c_{2}, c_{3}$ e $\mathcal{P}$ são dadas por:

$$
\begin{aligned}
& \frac{\partial l\left(c_{1}, c_{2}, c_{3}, \mathcal{P}\right)}{\partial c_{1}}=\frac{r}{c_{1}}+\frac{n}{c_{123}}+\frac{n-r}{c_{13}}-\frac{n}{c_{12}}-S_{X}(\mathcal{P}) \\
& \frac{\partial l\left(c_{1}, c_{2}, c_{3}, \mathcal{P}\right)}{\partial c_{2}}=\frac{n-r}{c_{2}}+\frac{n}{c_{123}}+\frac{r}{c_{23}}-\frac{n}{c_{12}}-S_{Y}(\mathcal{P}) \\
& \frac{\partial l\left(c_{1}, c_{2}, c_{3}, \mathcal{P}\right)}{\partial c_{3}}=\frac{n}{c}+\frac{r}{c_{23}}+\frac{n-r}{c_{13}}-T(\mathcal{P}) \\
& \frac{\partial l\left(c_{1}, c_{2}, c_{3}, \mathcal{P}\right)}{\partial \mathcal{P}}=2 \sum_{j=1}^{J} n_{j} \log V_{j}-c_{1} \sum_{j=1}^{n} n_{j} \bar{x}_{j} V_{j}^{\mathcal{P}} \log V_{j}- \\
& c_{2} \sum_{j=1}^{n} n_{j} \bar{y}_{j} V_{j}^{\mathcal{P}} \log V_{j}-c_{3} \sum_{j=1}^{n} R_{j} V_{j}^{\mathcal{P}} \log V_{j}
\end{aligned}
$$

Resolvendo-se o sistema de equações simultâneas (7.8) para $c_{1}, c_{2}$, $c_{3}$ e $\mathcal{P}$ encontram-se os EMV dos parâmetros. 


$$
\left\{\begin{array}{l}
\frac{\partial l\left(c_{1}, c_{2}, c_{3}, \mathcal{P}\right)}{\partial c_{1}}=0 \\
\frac{\partial l\left(c_{1}, c_{2}, c_{3}, \mathcal{P}\right)}{\partial c_{2}}=0 \\
\frac{\partial l\left(c_{1}, c_{2}, c_{3}, \mathcal{P}\right)}{\partial c_{3}}=0 \\
\frac{\partial l\left(c_{1}, c_{2}, c_{3}, \mathcal{P}\right)}{\partial \mathcal{P}}=0
\end{array}\right.
$$

Visto que não é possível encontrar uma forma analítica fechada para $c_{1}, c_{2}, c_{3}$ e $\mathcal{P}$ deve-se empregar um método numérico para resolver o sistema de equações simultâneas (7.8).

\subsubsection{Inferências sobre os parâmetros $c_{1}, c_{2}, c_{3}$ e $\mathcal{P}$.}

Para fazer inferências sobre os parâmetros, utiliza-se a aproximação normal assintótica dos estimadores de máxima verossimilhança dada por:

$$
\left(\hat{c_{1}}, \hat{c_{2}}, \hat{c_{3}}, \hat{\mathcal{P}}\right) \stackrel{a}{\sim} N_{3}\left[\left(c_{1}, c_{2}, c_{3}, \mathcal{P}\right) ; \mathcal{I}^{-1}\right]
$$

onde $\mathcal{I}$ é a matriz de informação observada, isto é, a matriz das derivadas parciais de segunda ordem do logaritmo da função verossimilhança $l\left(c_{1}, c_{2}, c_{3}, \mathcal{P}\right)$ multiplicados por $(-1)$.

\subsubsection{Intervalos de Confiança}

Considerando-se a normalidade assintótica dos estimadores de máxima verossimilhança $\hat{c_{1}}, \hat{c_{2}}, \hat{c_{3}}$ e $\hat{\mathcal{P}}$ podem-se calcular os intervalos de confiança para os parâmetros $c_{1}, c_{2}, c_{3}$ e $\mathcal{P}$.

Representando a inversa da matriz de informação observada por: 


$$
\mathcal{I}^{-1}=\left[\begin{array}{llll}
b_{11} & b_{12} & b_{13} & b_{14} \\
b_{21} & b_{22} & b_{23} & b_{24} \\
b_{31} & b_{32} & b_{33} & b_{34} \\
b_{41} & b_{42} & b_{43} & b_{44}
\end{array}\right]
$$

e considerando um nível de confiança $100(1-\alpha) \%$, os intervalos de confiança para $c_{1}, c_{2}, c_{3}$ e $\mathcal{P}$ são dados por:

$$
\begin{aligned}
& i c\left(c_{1}\right)=\left[\hat{c_{1}}-z_{\alpha / 2}\left(\hat{b}_{11}\right)^{1 / 2} ; \hat{c}_{1}+z_{\alpha / 2}\left(\hat{b}_{11}\right)^{1 / 2}\right] \\
& i c\left(c_{2}\right)=\left[\hat{c_{2}}-z_{\alpha / 2}\left(\hat{b}_{22}\right)^{1 / 2} ; \hat{c_{2}}+z_{\alpha / 2}\left(\hat{b}_{22}\right)^{1 / 2}\right] \\
& i c\left(c_{3}\right)=\left[\hat{c_{3}}-z_{\alpha / 2}\left(\hat{b}_{33}\right)^{1 / 2} ; \hat{c_{3}}+z_{\alpha / 2}\left(\hat{b}_{33}\right)^{1 / 2}\right] \\
& i c(\mathcal{P})=\left[\hat{\mathcal{P}}-z_{\alpha / 2}\left(\hat{b}_{44}\right)^{1 / 2} ; \hat{\mathcal{P}}+z_{\alpha / 2}\left(\hat{b}_{44}\right)^{1 / 2}\right]
\end{aligned}
$$

onde $z_{\alpha / 2}$ é o quantil correspondente da distribuição normal padronizada, e $\hat{b}_{i j}$ é o elemento da inversa da matriz de informação observada $\mathcal{I}$ avaliado nos estimadores de máxima verossimilhança.

\subsubsection{Confiabilidade de sistemas}

Se os tempos de vida de um sistema de dois componentes submetidos a testes acelerados seguem uma distribuição com densidade (2.1), os tempos médios de vida $\mu_{1}^{j}=E(X)$ e $\mu_{2}^{j}=E(Y)$ desses componentes num dado nível de estresse $j$ são dados por:

$$
\begin{aligned}
& \mu_{1}^{j}=\frac{c_{123} c_{12}+c_{2} c_{3}}{c_{123} c_{12} c_{13}} V_{j}^{-\mathcal{P}} \\
& \mu_{2}^{j}=\frac{c_{123} c_{12}+c_{1} c_{3}}{c_{123} c_{12} c_{23}} V_{j}^{-\mathcal{P}}
\end{aligned}
$$


Assumindo-se um sistema composto por dois componentes, ligados em série, tem-se que o sistema funciona se os dois componentes funcionam simultaneamente; assumindo-se ainda que eles têm distribuição de vida com função de densidade dada por (2.1), a função de confiabilidade do sistema no tempo $t_{0}$ num dado nivel de estresse $j$ é dada por:

$$
R_{S}^{j}\left(t_{0}\right)=P\left[\min (X, Y)>t_{0}\right]=e^{-c_{123} V_{j}^{p} t_{0}}
$$

Considerando-se o mesmo sistema onde os componentes estão ligados em paralelo e possuem distribuição de vida com função de densidade (2.1), a função de confiabilidade do sistema no tempo $t_{0}$ num dado nível de estresse $j$ é dada por:

$$
R_{P}^{j}\left(t_{0}\right)=P\left[\max (X, Y)>t_{0}\right]=\frac{c_{123}\left(e^{c_{1} V_{j}^{\mathcal{P}} t_{0}}+e^{c_{2} V_{j}^{p} t_{0}}-1\right)-c_{3}}{c_{12} e^{c_{123} V_{j}^{p} t_{0}}}
$$

Inferências para a confiabilidade de sistemas num teste acelerado são, em geral, obtidas via resultados assintóticos dos estimadores de máxima verossimilhança. A partir da normalidade assintótica dos estimadores de máxima verossimilhança para $c_{1}, c_{2}, c_{3}$ e $\mathcal{P}$ pode-se usar o método "delta" (ver por exemplo, MILlER, 1982) para obter uma distribuição assintótica para a função de confiabilidade num dado nível de estresse $V_{j}, j=1, \cdots, J$. Em geral a precisão desses resultados assintóticos pode não ser boa, especialmente para tamanhos amostrais pequenos ou moderados. 


\subsection{Análise Bayesiana usando Gibbs com Metro- polis-Hastings}

Nesta secção considera-se uma análise Bayesiana do modelo ACBVE (2.1) em testes de vida acelerados com o modelo de potência utilizando-se o algoritmo de "Gibbs Sampling" associado ao algoritmo de "Metropolis-Hastings".

Considera-se a introdução da variável latente $N_{1}$ representando o número de observações tais que $X_{i 1}<Y_{i 1}$, e assumem-se as seguintes densidades $a$ priori para $N_{1}, c_{1}, c_{2}, c_{3}$ e $\mathcal{P}$ :

$$
\begin{aligned}
& N_{1} \sim b\left(n_{1}, c_{1} / c_{12}\right) \\
& c_{1} \sim \Gamma\left(a_{1}, b_{1}\right), \quad a_{1} \text { e } b_{1} \text { conhecidos } \\
& c_{2} \sim \Gamma\left(a_{2}, b_{2}\right), a_{2} \text { e } b_{2} \text { conhecidos } \\
& c_{3} \sim \Gamma\left(a_{3}, b_{3}\right), a_{3} \text { e } b_{3} \text { conhecidos } \\
& \mathcal{P} \sim N\left(\mu_{0}, \sigma_{0}^{2}\right), \mu_{0}, \sigma_{0}^{2} \text { conhecidos. }
\end{aligned}
$$

Observe que $N_{1}$ tem distribuição binomial com probabilidade de sucesso,

$$
P\left(X_{i 1}<Y_{i 1}\right)=\frac{\lambda_{1}}{\lambda_{12}}=\frac{c_{1} V_{1}^{\mathcal{P}}}{c_{1} V_{1}^{\mathcal{P}}+c_{2} V_{2}^{\mathcal{P}}}=\frac{c_{1}}{c_{12}}
$$

$N\left(\mu_{0}, \sigma_{0}^{2}\right)$ denota a distribuição normal com média $\mu_{0}$ e variância $\sigma_{0}^{2}, \Gamma\left(a_{1}, b_{1}\right)$ denota uma distribuição gama com média $a_{i} / b_{i}$ e variância $a_{i} / b_{i}^{2}$. Assumindo-se independência entre os parâmetros $N_{1}, c_{1}, c_{2}, c_{3}$ e $\mathcal{P}$ a densidade a posteriori conjunta é dada por,

$$
\pi\left(N_{1}, c_{1}, c_{2}, c_{3}, \mathcal{P} \mid \mathcal{D}\right) \propto
$$




$$
\begin{aligned}
& \left(\begin{array}{c}
n \\
N_{1}
\end{array}\right)\left(\frac{c_{1}}{c_{12}}\right)^{N_{1}}\left(\frac{c_{2}}{c_{12}}\right)^{n_{1}-N_{1}} \frac{1}{c_{12}^{n}} \\
& c_{1}^{r+a_{1}-1} c_{2}^{n-r+a_{2}-1} c_{3}^{a_{3}-1} c_{23}^{r} c_{13}^{n-r} c_{123}^{n} \\
& \left\{\prod_{j=1}^{J}\left(V_{j}^{2 \mathcal{P}}\right)^{n_{j}}\right\} \exp \left\{-\frac{1}{2 \sigma_{0}^{2}}\left(\mathcal{P}-\mu_{0}\right)^{2}\right\} \\
& \exp \left\{-\left[b_{1}+S_{X}(\mathcal{P})\right] c_{1}-\left[b_{2}+S_{Y}(\mathcal{P})\right] c_{2}-\left[b_{3}+T(\mathcal{P}] c_{3}\right\}\right.
\end{aligned}
$$

onde $\mathcal{D}$ denota o conjunto de dados.

As densidades a posteriori condicionais cheias necessárias para o algoritmo Gibbs Sampling, são dadas por,

$$
\begin{aligned}
N_{1} \mid c_{1}, c_{2}, c_{3}, \mathcal{D} & \propto b\left(n_{1}, \frac{c_{1}}{c_{12}}\right) \\
\pi\left(c_{1} \mid N_{1}, c_{2}, c_{3}, \mathcal{P}, \mathcal{D}\right) \propto & \frac{c_{1}^{N_{1}+r+a_{1}-1} c_{13}^{n-r} c_{123}^{n}}{c_{12}^{n+n_{1}} \exp \left\{-\left[b_{1}+S_{X}(\mathcal{P})\right] c_{1}\right\}} \\
\pi\left(c_{2} \mid N_{1}, c_{1}, c_{3}, \mathcal{P}, \mathcal{D}\right) \propto & \frac{c_{2}^{n_{1}-N_{1}+n-r+a_{2}-1} c_{23}^{r} c_{123}^{n}}{c_{12}^{n+n_{1}}} \exp \left\{-\left[b_{2}+S_{Y}(\mathcal{P})\right] c_{2}\right\} \\
\pi\left(c_{3} \mid N_{1}, c_{1}, c_{2}, \mathcal{P}, \mathcal{D}\right) \propto & c_{3}^{a_{3}-1} c_{23}^{r} c_{13}^{n-r} c_{123}^{n} \exp \left\{-\left[b_{3}+T(\mathcal{P})\right] c_{3}\right\} \\
\pi\left(\mathcal{P} \mid N_{1}, c_{1}, c_{2}, c_{3}, \mathcal{D}\right) \propto & \left\{\prod_{j=1}^{J}\left(V_{j}^{2 \mathcal{P}}\right)^{n_{j}}\right\} \exp \left\{-\frac{1}{2 \sigma_{0}^{2}}\left(\mathcal{P}-\mu_{0}\right)^{2}\right\} \\
& \exp \left\{-c_{1} S_{X}(\mathcal{P})-c_{2} S_{Y}(\mathcal{P})-c_{3} T(\mathcal{P})\right\}
\end{aligned}
$$

Observe que é necessário usar o algoritmo de Metropolis-Hastings para gerar as variáveis $c_{1}, c_{2}, c_{3}$ e $\mathcal{P}$. Pode-se monitorar a convergência das amostras Gibbs usando-se o método proposto por Gelman \& RUBin (1992).

\subsubsection{Estimadores de Bayes para os tempos de vida médios}

Considerando-se as amostras Gibbs geradas por (7.17) para 
$N_{1}, c_{1}, c_{2}, c_{3}$ e $\mathcal{P}$ podem-se obter as as estimativas de Monte Carlo para os momentos a posteriori de interesse. Um caso especial é dado pelo tempo de vida médio em um nível especificado de estresse:

$$
\begin{aligned}
& \mu_{1}^{j}=E(X)=\frac{\left(c_{123} c_{12}+c_{2} c_{3}\right)}{c_{123} c_{12} c_{13}} V_{j}^{-\mathcal{P}} \\
& \mu_{2}^{j}=E(Y)=\frac{\left(c_{123} c_{12}+c_{1} c_{3}\right)}{c_{123} c_{12} c_{23}} V_{j}^{-\mathcal{P}}
\end{aligned}
$$

Estimadores de Bayes para $\mu_{i}^{j}, i=1,2, j=1, \ldots, J$ com relação à função de perda quadrática, em um nível de estresse especificado $j$ são dadas por $E\left(\mu_{i}^{j} \mid \mathcal{D}\right)$ que podem ser aproximadas por estimativas de Monte Carlo, na forma,

$$
\begin{aligned}
& {\hat{\mu_{1}}}^{j}=\frac{2}{R S} \sum_{s=1}^{S} \sum_{r=R / 2+1}^{R} \frac{c_{123}^{(r, s)} c_{12}^{(r, s)}+c_{2}^{(r, s)} c_{3}^{(r, s)}}{c_{12}^{(r, s)} c_{13}^{(r, s)} c_{123}^{(r, s)}} V_{j}^{\mathcal{P}(r, s)} \\
& {\hat{\mu_{2}}}^{j}=\frac{2}{R S} \sum_{s=1}^{S} \sum_{r=R / 2+1}^{R} \frac{c_{123}^{(r, s)} c_{12}^{(r, s)}+c_{1}^{(r, s)} c_{3}^{(r, s)}}{c_{12}^{(r, s)} c_{23}^{(r, s)} c_{123}^{(r, s)}} V_{j}^{\mathcal{P}(r, s)}
\end{aligned}
$$

onde $c_{123}^{(r, s)}=c_{1}^{(r, s)}+c_{2}^{(r, s)}+c_{3}^{(r, s)}, c_{12}^{(r, s)}=c_{1}^{(r, s)}+c_{2}^{(r, s)}, c_{13}^{(r, s)}=c_{1}^{(r, s)}+c_{3}^{(r, s)}, c_{23}^{(r, s)}=$ $c_{2}^{(r, s)}+c_{3}^{(r, s)}$ e $c_{1}^{(r, s)}, c_{2}^{(r, s)}$ e $c_{1}^{(r, s)}$ denotam os valores obtidos para $c_{1}, c_{2}, c_{3}$ e $\mathcal{P}$ na $r$-ésima iteração e na $s$-ésima replicação onde $R$ e $S$ são respectivamente, o número total de iterações e o número total de replicações da amostra Gibbs.

\subsubsection{Estimadores de Bayes para a função de confiabilida- de}

Assumindo-se o modelo ACBVE (2.1) com parâmetros $\lambda_{1 j}, \lambda_{2 j} \mathrm{e}$ $\lambda_{3 j}, j=1, \ldots, J$ e considerando-se o modelo de potência (7.1), a função de confiabi-lidade para um sistema com dois componentes em um nível especificado de estresse e num determinado tempo $t_{0}$ é dada por 


$$
\begin{array}{ll}
R_{S}^{j}\left(t_{0}\right)=\exp \left\{-c_{123} V_{j}^{\mathcal{P}} t_{0}\right\} & \text { para um sistema em série. } \\
R_{P}^{j}\left(t_{0}\right)=\frac{\left\{c_{123}\left(e^{c_{1} V_{j}^{\mathcal{P}} t_{0}}+e^{c_{2} V_{j}^{\mathcal{P}} t_{0}}-1\right)-c_{3}\right\}}{c_{12} e^{c_{123} V_{j}^{\mathcal{P}} t_{0}}} & \text { para um sistema em paralelo } .
\end{array}
$$

Estimadores de Bayes para $R_{S}^{j}\left(t_{0}\right)$ e $R_{P}^{j}\left(t_{0}\right)$ com relação à função de perda quadrática são dados por $E\left\{R_{S}^{j}\left(t_{0}\right) \mid \mathcal{D}\right\}$ e $E\left\{R_{P}^{j}\left(t_{0}\right) \mid \mathcal{D}\right\}$. Baseadas em amostradores de Gibbs com $R$ iterações e $S$ replicações, estimativas de Monte Carlo para esses momentos a posteriori são dadas por

$$
\hat{R}_{S}{ }^{j}\left(t_{0}\right)=\frac{2}{R S} \sum_{i=1}^{S} \sum_{\tau=R / 2+1}^{R} \exp \left\{-c_{123}^{(r, s)} V_{j}^{\mathcal{p}(r, s)} t_{0}\right\}
$$

para um sistema em série.

$$
{\hat{R_{P}}}^{j}\left(t_{0}\right)=\frac{2}{R S} \sum_{s=1}^{S} \sum_{r=R / 2+1}^{R} \frac{\left\{c_{123}^{(r, s)}\left(e^{c_{1}^{(r, s)} V_{j}^{p(r, s)} t_{0}}+e^{c_{2}^{(r, s)} V_{j}^{p(r, s)} t_{0}}-1\right)-c_{3}^{(r, s)}\right\}}{c_{12}^{(r, s)} e^{c^{(r, s)} V_{j}^{\mathcal{p}(r, s)} t_{0}}}
$$

para um sistema em paralelo. 


\section{Capítulo 8}

\section{Modelo de regressão com dados de sobrevivência bivariados}

No Capítulo 7 considera-se a inclusão de um único fator de estresse como variável concomitante. Ocorre que, usualmente, o tempo de vida de produtos pode ser afetado por vários fatores de estresse e um modelo mais complexo é necessário na prática. Existem muitos problemas nos quais o conhecimento de mais de uma variável independente é necessário a fim de se obter um melhor entendimento e/ou melhor predição de uma variável resposta.

Seja $(X, Y)$ um vetor aleatório não negativo representando o tempo de vida de cada unidade com dois componentes com função densidade dada por (2.1) e considere o modelo dado por (8.1)

$$
\begin{aligned}
& \lambda_{1 j}=c_{1} \exp \left(\beta_{1} V_{1 j}+\beta_{2} V_{2 j}+\ldots+\beta_{K} V_{K j}\right) \\
& \lambda_{2 j}=c_{2} \exp \left(\beta_{1} V_{1 j}+\beta_{2} V_{2 j}+\ldots+\beta_{K} V_{K j}\right) \\
& \lambda_{3 j}=c_{3} \exp \left(\beta_{1} V_{1 j}+\beta_{2} V_{2 j}+\ldots+\beta_{K} V_{K j}\right)
\end{aligned}
$$


onde $V_{k j}, k=1, \ldots, K$ são $K$ variáveis explanatórias e $c_{1}, c_{2}, c_{3}, \beta_{1}, \ldots, \beta_{K}$ são constantes.

Considerando $n_{j}$ unidades $\left(X_{1 j}, Y_{1 j}\right),\left(X_{2 j}, Y_{2 j}\right), \ldots,\left(X_{n_{j} j}, Y_{n_{j} j}\right)$, no início de cada teste com condição de estresse $\left(V_{1 j}, V_{2 j}, \ldots, V_{K j}\right)$ onde $j=1, \ldots, J$, a função de verossimilhança para $c_{1}, c_{2}, c_{3}, \beta_{1}, \ldots, \beta_{K}$ é dada por:

$$
L_{j}\left(c_{1}, c_{2}, c_{3}, \beta_{1}, \beta_{2}, \ldots, \beta_{K}\right)=\prod_{i=1}^{n_{j}} f_{1}^{\delta_{i j}}\left(X_{i j}, Y_{i j}\right) f_{2}^{1-\delta_{i j}}\left(X_{i j}, Y_{i j}\right)
$$

onde $\delta_{i j}=1$ se $X_{i j}<Y_{i j}$ e $\delta_{i j}=0$ se $X_{i j} \geq Y_{i j}$, e

$$
\begin{aligned}
& f_{1}\left(X_{i j}, Y_{i j}\right)=\frac{c_{1} c_{123} c_{23}}{c_{12}} \exp \left\{2 \sum_{k=1}^{K} \beta_{k} V_{k j}\right\} \exp \left\{-\left(c_{1} X_{i j}+c_{23} Y_{i j}\right)\right. \\
& \left.\exp \left\{\sum_{k=1}^{K} \beta_{k} V_{k j}\right\}\right\} \quad \text { se } \quad X_{i j}<Y_{i j} \\
& f_{2}\left(X_{i j}, Y_{i j}\right)=\frac{c_{2} c_{123} c_{13}}{c_{12}} \exp \left\{2 \sum_{k=1}^{K} \beta_{k} V_{k j}\right\} \exp \left\{-\left(c_{13} X_{i j}+c_{2} Y_{i j}\right)\right. \\
& \left.\exp \left\{\sum_{k=1}^{K} \beta_{k} V_{k j}\right\}\right\} \quad \text { se } \quad X_{i j} \geq Y_{i j}
\end{aligned}
$$

onde $c_{12}=c_{1}+c_{2}, \quad c_{13}=c_{1}+c_{3}, \quad c_{23}=c_{2}+c_{3} \quad \mathrm{e} \quad c_{123}=c_{1}+c_{2}+c_{3}$.

Isto é,

$$
\begin{gathered}
L_{j}\left(c_{1}, c_{2}, c_{3}, \beta_{1}, \beta_{2}, \ldots, \beta_{K}\right)=\frac{c_{1}^{r_{j}} c_{123}^{n_{j}} c_{23}^{r_{j}} c_{2}^{n_{j}-r_{j}} c_{13}^{n_{j}-r_{j}}}{c_{12}^{n_{j}}} \exp \left\{2 n_{j} \sum_{k=1}^{K} \beta_{k} V_{k j}\right\} \\
\exp \left\{-\left(c_{1} n_{j} \bar{X}_{j}+c_{2} n_{j} \bar{Y}_{j}+c_{3} R_{j}\right)\right. \\
\left.\exp \left\{\sum_{k=1}^{K} \beta_{k} V_{k j}\right\}\right\}
\end{gathered}
$$


$n_{j} \bar{X}_{j}=\sum_{i=1}^{n_{j}} X_{i j} ; \quad n_{j} \bar{Y}_{j}=\sum_{i=1}^{n_{j}} Y_{i j} ; \quad R_{j}=\sum_{i=1}^{n_{j}}\left[\delta_{i j} Y_{i j}+\left(1-\delta_{i j}\right) X_{i j}\right]$

Assumindo que os dados obtidos nas $\mathrm{J}$ condições de estresse $\left(V_{1 j}, V_{2 j}, \ldots, V_{K j}\right)$ onde $j=1, \ldots, J$, são independentes, a função de verossimilhança para $c_{1}, c_{2}, c_{3}, \beta_{1}, \ldots, \beta_{K}$ é dada por:

$$
L\left(c_{1}, c_{2}, c_{3}, \beta_{1}, \beta_{2}, \ldots, \beta_{K}\right)=\prod_{i=1}^{J} L_{j}\left(c_{1}, c_{2}, c_{3}, \beta_{1}, \beta_{2}, \ldots, \beta_{K}\right)
$$

Isto é,

$$
\begin{aligned}
& L\left(c_{1}, c_{2}, c_{3}, \beta_{1}, \beta_{2}, \ldots, \beta_{K}\right)=\frac{c_{1}^{r} c_{123}^{n} c_{23}^{r} c_{2}^{n-r} c_{13}^{n-r}}{c_{12}^{n}} \\
& \quad \exp (Z(\theta)) \exp \left\{-\left(c_{1} S_{X}(\theta)+c_{2} S_{Y}(\theta)+c_{3} T(\theta)\right\}\right.
\end{aligned}
$$

onde

( i) $r=\sum_{j=1}^{J} r_{j}, \quad n=\sum_{j=1}^{J} n_{j}$

(ii) $Z(\theta)=2 n \sum_{k=1}^{K} \beta_{k} V_{k j}$,

(iii) $S_{X}(\boldsymbol{\theta})=\sum_{j=1}^{J} n_{j} \bar{X}_{j} \exp \left\{\sum_{k=1}^{K} \beta_{k} V_{k j}\right\}$,

(iv) $S_{Y}(\theta)=\sum_{j=1}^{J} n_{j} \bar{Y}_{j} \exp \left\{\sum_{k=1}^{K} \beta_{k} V_{k j}\right\}$

( v) $T(\theta)=\sum_{j=1}^{J} R_{j} \exp \left\{\sum_{k=1}^{K} \beta_{k} V_{k j}\right\}$.

Para a obtenção de estimadores de máxima verossimilhança, podese ter grande dificuldade computacional na obtenção do ponto onde (8.6) atinge o valor máximo. Além disso, a determinação da distribuição normal assintótica dos estimadores de máxima verossimilhança baseada na informação de Fisher pode ser dif́́cil de se obter e de precisão ruim. O uso de métodos Bayesianos para 
este caso pode ser de grande simplificação, especialmente usando algoritmos Gibbscom-Metropolis-Hastings. O uso de outras técnicas computacionais como método numéricos ou de aproximação de Laplace, podem ser inviáveis neste caso.

\subsection{Análise Bayesiana usando Gibbs com Metro- polis-Hastings}

Para inferência Bayesiana, considera-se a introdução de uma variável latente $N_{1}$ representando o número de observações tais que $X_{i 1}<Y_{i 1}$, e assume-se a seguinte densidade a priori para $N_{1}, c_{1}, c_{2}, c_{3}$, e $\beta_{k}$ :

$$
\begin{aligned}
N_{1} & \sim b\left(n_{1}, c_{1} / c_{12}\right) \\
c_{1} & \sim \Gamma\left(a_{1}, b_{1}\right), a_{1} \text { e } b_{1} \text { conhecidos } \\
c_{2} & \sim \Gamma\left(a_{2}, b_{2}\right), a_{2} \text { e } b_{2} \text { conhecidos } \\
c_{3} & \sim \Gamma\left(a_{3}, b_{3}\right), a_{3} \text { e } b_{3} \text { conhecidos } \\
\beta_{k} & \sim N\left(\mu_{0 k}, \sigma_{0 k}^{2}\right), \mu_{0 i}, \sigma_{0 i}^{2} \text { conhecidos e } k=1, \ldots, K .
\end{aligned}
$$

Observe que $N_{1}$ tem distribuição binomial com probabilidade de sucesso,

$$
P\left(X_{i 1}<Y_{i 1}\right)=\frac{c_{1}}{c_{12}}
$$

$N\left(\mu_{0 i}, \sigma_{0 i}^{2}\right)$ denota a distribuição normal com média $\mu_{0 i}$ e variância $\sigma_{0 i}^{2}$ e $\Gamma\left(a_{i}, b_{i}\right)$ denota a distribuição gama com média $a_{i} / b_{i}$ e variância $a_{i} / b_{i}^{2}$. Ainda, assumindo-se independência entre os parâmetros $N_{1}, c_{1}, c_{2}, c_{3}, \beta_{k}, k=1, \ldots, K$, a densidade $a$ posteriori conjunta é dada por, 


$$
\begin{aligned}
& \pi\left(N_{1}, c_{1}, c_{2}, c_{3}, \beta_{1}, \ldots, \beta_{K} \mid \mathcal{D}\right) \propto \\
& \left(\begin{array}{l}
n_{1} \\
N_{1}
\end{array}\right)\left(\frac{c_{1}}{c_{12}}\right)^{N_{1}}\left(\frac{c_{2}}{c_{12}}\right)^{n_{1}-N_{1}} \\
& \frac{c_{1}^{r+a_{1}-1} c_{2}^{n-r+a_{2}-1} c_{3}^{a_{3}-1} c_{23}^{\tau} c_{13}^{n-\tau} c_{123}^{n}}{c_{12}^{n}} \\
& \prod_{i=1}^{K} \exp \left\{-\frac{1}{2 \sigma_{0 i}^{2}}\left(\beta_{i}-\mu_{0 i}\right)^{2}\right\} \\
& \exp (Z(\boldsymbol{\theta})) \exp \left\{-\left[b_{1}+S_{X}(\boldsymbol{\theta})\right] c_{1}-\left[b_{2}+S_{Y}(\boldsymbol{\theta})\right] c_{2}-\left[b_{3}+T(\boldsymbol{\theta})\right] c_{3}\right\}
\end{aligned}
$$

onde $\mathcal{D}$ denota o conjunto de dados.

As densidades a posteriori condicionais cheias para o algoritmo Gibbs são dadas por:

$$
\begin{aligned}
& N_{1} \mid c_{1}, c_{2}, c_{3}, \beta_{1}, \ldots, \beta_{K}, \mathcal{D} \sim b\left(n_{1}, \frac{c_{1}}{c_{12}}\right) \\
& \pi\left(c_{1} \mid N_{1}, c_{2}, c_{3}, \beta_{1}, \ldots, \beta_{K}, \mathcal{D}\right) \propto \\
& \frac{c_{1}^{N_{1}+r+a_{1}-1} c_{13}^{n-r} c_{123}^{n}}{c_{12}^{n+n_{1}} \exp \left\{-\left[b_{1}+S_{X}(\boldsymbol{\theta})\right] c_{1}\right\}} \\
& \pi\left(c_{2} \mid N_{1}, c_{1}, c_{3}, \beta_{1}, \ldots, \beta_{K}, \mathcal{D}\right) \propto \\
& \quad \frac{c_{2}^{n_{1}-N_{1}+n-r+a_{2}-1} c_{23}^{r} c_{123}^{n}}{c_{12}^{n+n_{1}}} \exp \left\{-\left[b_{2}+S_{Y}(\boldsymbol{\theta})\right] c_{2}\right\} \\
& \pi\left(c_{3} \mid N_{1}, c_{1}, c_{2}, \beta_{1}, \ldots, \beta_{K}, \mathcal{D}\right) \propto \\
& \quad c_{3}^{a_{3}-1} c_{23}^{r} c_{13}^{n-r} c_{123}^{n} \exp \left\{-\left[b_{3}+T(\theta)\right] c_{3}\right\} \\
& \pi\left(\beta_{i} \mid N_{1}, c_{1}, c_{2}, c_{3}, \beta_{1}, \ldots, \beta_{i-1}, \beta_{i+1}, \ldots, \beta_{K}, \mathcal{D}\right) \propto
\end{aligned}
$$




$$
\begin{aligned}
& \exp (Z(\boldsymbol{\theta})) \exp \left\{-\frac{1}{2 \sigma_{0 i}^{2}}\left(\beta_{i}-\mu_{0 i}\right)^{2}\right\} \\
& \exp \left\{-c_{1} S_{X}(\boldsymbol{\theta})-c_{2} S_{Y}(\boldsymbol{\theta})-c_{3} T(\boldsymbol{\theta})\right\}
\end{aligned}
$$

Observe que, é necessário usar o algoritmo de Metropolis-Hastings para gerar as variáveis $c_{1}, c_{2}, c_{3}, \beta_{k}, k=1, \ldots, K$

A partir das amostras geradas pelo algoritmo do amostrador de Gibbs, podemos obter inferências para os parâmetros ou funções desses parâmetros de interesse, usando métodos de Monte Carlo, como já foi visto nos capítulos anteriores deste trabalho. 


\section{Capítulo 9}

\section{Exemplos Ilustrativos}

Neste capítulo apresentam-se alguns exemplos de aplicações com o objetivo de ilustrar a metodologia apresentada.

\subsection{Exemplo 01}

Este exemplo ilustra a metodologia apresentada no Capítulo 2, 3, 4 e 5. Considera-se a geração de $n=100$ observações de tempos de vida bivariados $\left(X_{i}, Y_{i}\right), i=1,2, \ldots, 100$ assumindo a distribuição ACBVE $(2.1) \operatorname{com} \lambda_{1}=0,08, \lambda_{2}=$ $0,06 \quad$ e $\quad \lambda_{3}=0,06$. Assim, $\lambda_{12}=0,14, \quad \lambda_{13}=0,14, \quad \lambda_{23}=0,12, \quad \lambda=0,2$, $E(X)=8,0612, \quad E(Y)=9,7619, \quad \sigma_{X}=7,7046, \quad \sigma_{Y}=9,0382 \quad$ e $\quad \rho_{X Y}=0,1497$ segundo (2.3) e (2.4).

Dos passos (1) e (2) do Capítulo 4, primeiro geram-se $N=1000$ observações $W_{i}=\min \left(X_{i}, Y_{i}\right)$ da densidade exponencial com parâmetro $\lambda=0,20 \mathrm{e}$ também $N=1000$ variáveis indicadoras $\delta_{i}$ da distribuição Bernoulli com parâmetro $p=\frac{\lambda_{1}}{\lambda_{12}}=0,5714$ segundo (4.2). Seguindo-se o passo (3) do Capítulo 4, obtêm-se os dados de tempo de vida bivariados $\left(X_{i}, Y_{i}\right)$, usando o valor predito para $X_{i}$ ou $Y_{i}$, dependendo do valor obtido para $\delta_{i}, i=1,2, \ldots, 1000$. Isto é, se $\delta_{i}=1$, tem-se 
Tabela 9.1: Dados de tempo de vida bivariados gerados $\lambda_{1}=0,08, \lambda_{2}=0,06$ e $\lambda_{3}=0,06$.

\begin{tabular}{|c|c|c|c|c|c|}
\hline$X$ & $Y$ & $X$ & $Y$ & $X$ & $Y$ \\
\hline 0,01590 & 9,7752 & 0,92239 & 10,5434 & 17,4887 & 9,9211 \\
\hline 0,03607 & 9,7921 & 95066 & 10,5677 & 4520 & 1,0002 \\
\hline 0,06805 & 9,8189 & 1,00951 & 10,6182 & 1,6665 & 11,1866 \\
\hline 0,08325 & 9,8316 & 1,03304 & 10,6384 & 1,8994 & 11,3900 \\
\hline 0,11342 & 9,8570 & 1,07366 & 10,6733 & 2,2443 & 11,6929 \\
\hline 0,13787 & 9,8775 & 1,10142 & 10,6972 & 2,6338 & 12,0372 \\
\hline 0,14587 & 9,8842 & 1,11178 & 10,7061 & 3,1124 & 12,4633 \\
\hline 0,20908 & 9,9374 & 1,14278 & 10,7328 & 3,4978 & 12,8087 \\
\hline 0,23578 & 9,9599 & 1,17866 & 10,7637 & 3,8483 & 13,1245 \\
\hline 0,28811 & 10,0040 & 1,20725 & 10,7884 & 4,3102 & 13,5430 \\
\hline 0,32763 & 10,0373 & 1,25401 & 10,8288 & 4,7532 & 13,9467 \\
\hline 0,35206 & 10,0580 & 8,08814 & 0,0292 & 5,3150 & 14,4614 \\
\hline 0,37796 & 10,0798 & 8,2762 & 0,2331 & 5,6941 & 14,8105 \\
\hline 0,39686 & 10,0958 & 8,5279 & 0,5053 & 6,1598 & 15,2411 \\
\hline 0,40914 & 10,1062 & 8,7821 & 0,7797 & 6,7099 & 15,7522 \\
\hline 0,41374 & 10,1101 & 9,0515 & 1,0697 & 7,5385 & 16,5264 \\
\hline 0,42769 & 10,1219 & 9,2952 & 1,3316 & 8,0414 & 16,9987 \\
\hline 0,44100 & 10,1332 & 15 & ,6169 & 8,6759 & 17,5969 \\
\hline 0,46664 & 10,1549 & 9,8797 & 3 & 9,4783 & 18,3567 \\
\hline 0,47383 & 10,1610 & 10,1831 & 2,2810 & 10,3666 & 19,2020 \\
\hline 0,50343 & 10,1861 & 10,4243 & 2,5378 & 11,1450 & 19,9458 \\
\hline 0,51873 & 10,1991 & 10,8824 & 3,0244 & 12,1213 & 20,8825 \\
\hline 0,53567 & 10,2135 & 11,3216 & 3,4897 & 18,6037 & 11,0695 \\
\hline 0,56358 & 10,2372 & 11,7383 & 3,9299 & 19,3300 & 11,8160 \\
\hline 0,61135 & 10,2778 & 2368 & & 0,1483 & 555 \\
\hline 0,64623 & 10 & & 4,9637 & & 77 \\
\hline 0,68866 & 10,3436 & 13,2373 & 0,0004 & 13,3829 & 22,0983 \\
\hline 0,70947 & 10,3614 & 14,0623 & 6,3677 & 14,4032 & 23,0855 \\
\hline 0,74952 & 10,3955 & ,0128 &, 000 & 15,1537 & 23,8137 \\
\hline 0,81160 & 10,4 & 15,3570 &, 7153 & 3,0677 & 31,5669 \\
\hline 0,85319 & 10,4842 & 15,4718 & 7,8345 & 31,8155 & 24,5140 \\
\hline 0,87853 & 10,5059 & 15,8906 & 8,2688 & 25,5306 & 33,9989 \\
\hline \multirow[t]{2}{*}{0,91137} & 10,5340 & 16,5681 & 8,9702 & 32,1644 & 40,5758 \\
\hline & & 17,1588 & $\mathbf{9 , 5 8 0 6}$ & & \\
\hline
\end{tabular}


$X_{i}<Y_{i}$ ou $W_{i}=X_{i}$ e calcula-se o valor predito para $Y_{i}$ usando (4.6). Se $\delta_{i}=0$, tem-se $X_{i} \geq Y_{i}$, isto é, $W_{i}=Y_{i}$ e achamos via (4.8), o valor predito para $X_{i}$.

Seguindo-se os passos (4) e (5) da Capítulo 4, escolhem-se $n=100$ observações $\left(X_{i}, Y_{i}\right)$ tais que a frequência observada das observações nas classes constrúdas esteja de acordo com a frequência esperada $n p_{j l}$ segundo (5.5).

$\mathrm{O}$ conjunto de dados obtido é apresentado na Tabela 9.1. Na Tabela 9.2 e na Figura 9.1, pode-se observar o bom ajuste entre as frequências esperadas e observadas dos dados de tempo de vida observados. Utilizando-se a Tabela 9.2 obtém-se um valor da estatística do teste $\chi^{2}$ de Pearson dado por $\chi_{\text {calc }}^{2}=20,92$.

Considerando-se uma amostra aleatória de tamanho $n=100 \mathrm{da}$ distribuição ACBVE (2.1) apresentada na Tabela 9.1, o logaritmo da função de verossimilhança para $\lambda_{1}, \lambda_{2}$ e $\lambda_{3}$ é dado por,

$$
\begin{aligned}
l\left(\lambda_{1}, \lambda_{2}, \lambda_{3}\right)= & 71 \ln \lambda_{1}+29 \ln \lambda_{2}+100 \ln \lambda+71 \ln \left(\lambda_{2}+\lambda_{3}\right)+ \\
& 29 \ln \left(\lambda_{1}+\lambda_{3}\right)-100 \ln \left(\lambda_{1}+\lambda_{2}\right)-674,45 \lambda_{1}- \\
& 1188,88 \lambda_{2}-1343,65 \lambda_{3}
\end{aligned}
$$

Considerando-se a expressão (9.1) e utilizando-se o método iterativo de Newton-Raphson tomando-se como valores iniciais $\lambda_{1}^{0}=0,04, \quad \lambda_{2}^{0}=0,03, \quad \lambda_{3}^{0}=$ 0,03 , acharam-se depois de 9 iterações, as estimativas de máxima verossimilhança $\hat{\lambda_{1}}=0,10782, \hat{\lambda_{2}}=0,04896, \hat{\lambda_{3}}=0,05396$, assim como os estimadores de máxima verossimilhança para funções de $\lambda_{1}, \lambda_{2}, \lambda_{3}$ como $\hat{E}(X)=6,6756, \hat{E}(Y)=11,4275$, $\hat{\sigma}_{X}=6,5311, \hat{\sigma}_{Y}=10,3788$, e $\rho_{X} Y=0,1241$ segundo (2.3) e (2.4) .

Para a geração dos dados de tempo de vida bivariados da Tabela 9.1 seguindo a distribuição ACBVE (2.1) implementou-se um programa utilizando o "software" SAS . (Programa 1)

Na Tabela 9.3 são apresentados os intervalos de confiança $95 \%$ para $\lambda_{1}, \lambda_{2}, \lambda_{3}$ considerando a parametrização $\left(\lambda_{1}, \lambda_{2}, \lambda_{3}\right)$ e as reparametrizações $\left(\theta_{1}, \theta_{2}, \theta_{3}\right)$ e $\left(\phi_{1}, \phi_{2}, \phi_{3}\right)$ onde $\theta_{1}, \theta_{2}$ e $\theta_{3}$ são dados por (5.3) e $\phi_{1}, \phi_{2}$ e $\phi_{3}$ 
Tabela 9.2: Distribuição de frequências ( $o=$ frequência observada: $e=$ frequência esperada)

\begin{tabular}{|l|l|l|l|l|}
\hline$\downarrow X Y \longrightarrow$ & $(0 ; 11]$ & $(11 ; 22]$ & $(22 ; 33]$ & $(33 ; 55]$ \\
\hline$(0,00 ; 7,500]$ & $\mathrm{e} 11=44,00$ & $\mathrm{o} 12=14,00$ & $\mathrm{o} 13=0,00$ & $\mathrm{o} 14=0,00$ \\
\hline & $\mathrm{e} 21=15,78$ & $\mathrm{e} 22=6,76$ & $\mathrm{e} 23=1,84$ & $\mathrm{e} 24=0,63$ \\
$(7,50 ; 15 ; 00]$ & $\mathrm{o} 21=17,00$ & $\mathrm{o} 22=7,00$ & $\mathrm{o} 23=2,00$ & $\mathrm{o} 24=0,00$ \\
\hline & $\mathrm{e} 31=5,49$ & $\mathrm{e} 32=2,83$ & $\mathrm{e} 33=1,01$ & $\mathrm{e} 34=0,34$ \\
$(15,00 ; 22,50]$ & $\mathrm{o} 31=7,00$ & $\mathrm{o} 32=3,00$ & $\mathrm{o} 33=1,00$ & $\mathrm{o} 34=0,00$ \\
\hline & $\mathrm{e} 41=1,92$ & $\mathrm{e} 42=0,99$ & $\mathrm{e} 43=0,49$ & $\mathrm{e} 44=0,19$ \\
& $\mathrm{o} 41=0,00$ & $\mathrm{o} 42=1,00$ & $\mathrm{o} 43=1,00$ & $\mathrm{o} 44=1,00$ \\
\hline$(22,50 ; 30,00]$ & $\mathrm{e} 51=0,91$ & $\mathrm{e} 52=0,47$ & $\mathrm{e} 53=0,24$ & $\mathrm{e} 54=0,14$ \\
\hline$(30,00 ; 37,50]$ & $\mathrm{o} 51=0,00$ & $\mathrm{o} 52=0,00$ & $\mathrm{o} 53=1,00$ & $\mathrm{o} 54=1,00$ \\
\hline
\end{tabular}

são dados por (5.4) (Transformação de Box \& Cox). Na Tabela 9.3 apresenta-se, também, o valor da medida de KASS \& Slate, STD. Verifica-se que a reparametrização que apresenta um menor valor para STD ou ainda, a reparametrização na qual a normalidade conjunta da função de verossimilhança é melhor, é obtida quando utiliza-se a reparametrização $\left(\phi_{1}, \phi_{2}, \phi_{3}\right)$.

Na reparametrização $\left(\phi_{1}, \phi_{2}, \phi_{3}\right)$, ou seja, utilizando-se a transformação de Box \& Cox considerando-se $\xi_{i}, i=1,2,3$ variando na escala

$$
\{-1,0 ;-0,8 ;-0,6 ;-0,4 ;-0,2 ; 0,0 ; 0,2 ; 0,4 ; 0,6 ; 0,8 ; 1,0\}
$$

calculam-se os $11^{3}$ valores de STD. $O$ valor apresentado na Tabela 9.3 é o menor valor assumido por STD e é obtido quando se considera $\xi_{1}=0,8, \xi_{2}=0,4, \xi_{3}=1,0$.

Considerando-se os dados de tempos de vida bivariados da Tabela 9.1 e a função de verossimilhança na parametrização $\left(\lambda_{1}, \lambda_{2}, \lambda_{3}\right)$ foram cons- 
Tabela 9.3: Intervalos de confiança $95 \%$ e STD

\begin{tabular}{cccc}
\hline \multicolumn{3}{c}{ Parametrização } \\
\hline EMV & $\left(\lambda_{1}, \lambda_{2}, \lambda_{3}\right)$ & $\left(\theta_{1}, \theta_{2}, \theta_{3}\right)$ & $\left(\phi_{1}, \phi_{2}, \phi_{3}\right)$ \\
\hline$\hat{\lambda_{1}}=0,1078$ & $(0,0605 ; 0,1551)$ & $(0,0695 ; 0,1672)$ & $(0,0628 ; 0,1570)$ \\
$\hat{\lambda_{2}}=0,0490$ & $(0,0164 ; 0,0815)$ & $(0,0252 ; 0,0952)$ & $(0,0226 ; 0,0883)$ \\
$\hat{\lambda_{3}}=0,0539$ & $(0,0073 ; 0,1006)$ & $(0,0227 ; 0,1282)$ & $(0,0073 ; 0,1006)$ \\
\hline STD & 0,7969 & 3,3590 & 0,02317 \\
\hline
\end{tabular}

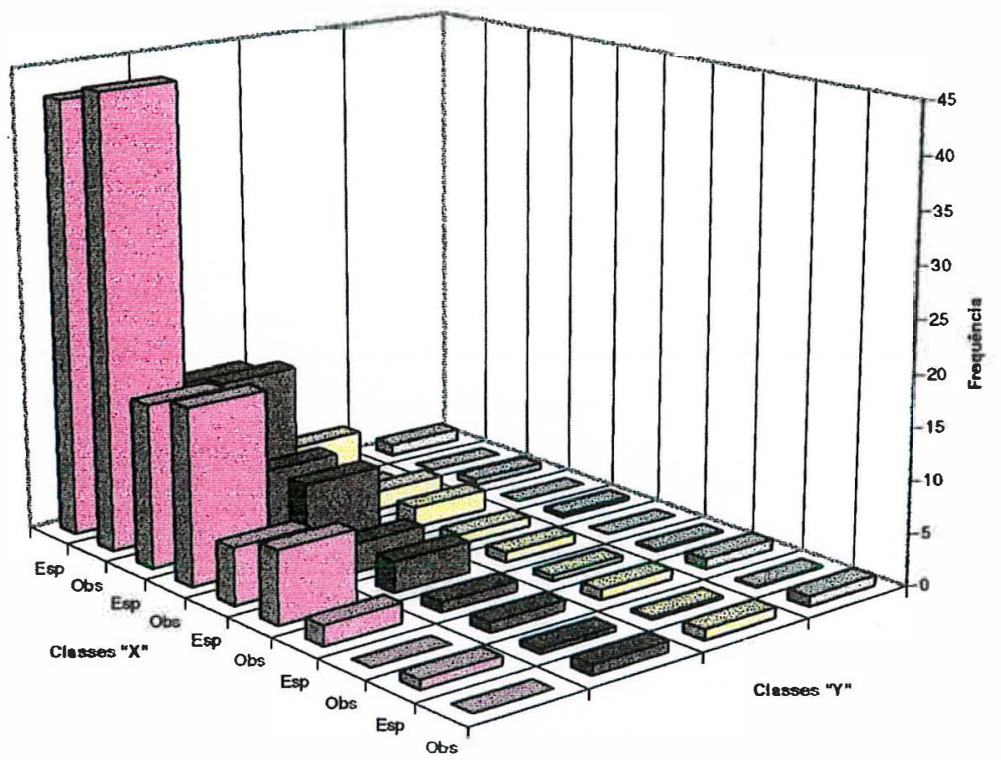

Figura 9.1: Distribuição das frequências esperadas (Esp) e das frequências observadas (Obs) segundo a Tabela 9.2 
truídos:

(a) Os gráficos da verossimilhança perfilada para os parâmetros: $\lambda_{1}, \lambda_{2}$ e $\lambda_{3}$ (Figura 9.2).

(b) 0 "t-plot" para os parâmetros: $\lambda_{1}, \lambda_{2}$ e $\lambda_{3}$. (Figura 9.3)

(c) Os contornos da verossimilhança perfilada para os parâmetros: $\left(\lambda_{1}, \lambda_{2}\right)$, $\left(\lambda_{1}, \lambda_{3}\right)$ e $\left(\lambda_{2}, \lambda_{3}\right)$. (Figura 9.4).

Na Figura 9.3 são apresentados os "t-plots" para os parâmetros $\lambda_{1}$, $\lambda_{2}$ e $\lambda_{3}$ utilizando-se os dados da Tabela 9.1. Pode-se observar através destes gráficos que para os parâmetros $\lambda_{1}$ e $\lambda_{3}$ o aspecto do gráfico é aproxidamente linear o mesmo não ocorrendo para o parâmetro $\lambda_{2}$ onde pode-se notar claramente uma não linearidade, mostrando assim que a verossimilhança perfilada para $\lambda_{2}$ não está "próxima" da normal. É interessante observar, também, na Figura 9.4 que o comportamento "não linear" observado na Figura 9.3 no "t-plot" para o parâmetro $\lambda_{2}$ "influencia" nos gráficos dos contornos das verossimilhanças perfiladas para $\left(\lambda_{1}, \lambda_{2}\right)$ $\left(\lambda_{2}, \lambda_{3}\right)$ onde se pode notar que as elipses apresentam uma dada curvatura.

Considerando-se os dados de tempos de vida bivariados da Tabela 9.1 e a função de verossimimilhança na parametrização $\left(\theta_{1}, \theta_{2}, \theta_{3}\right)$, foram construídos:

(a) Os gráficos da verossimilhança perfilada para os parâmetros: $\theta_{1}, \theta_{2}$ e $\theta_{3}$. (Figura 9.5)

(b) 0 "t-plot" para os parâmetros: $\theta_{1}, \theta_{2}$ e $\theta_{3}$. (Figura 9.6)

(c) Os contornos da verossimilhança perfilada para os parâmetros: $\left(\theta_{1}, \theta_{2}\right)$, $\left(\theta_{1}, \theta_{3}\right)$ e $\left(\theta_{2}, \theta_{3}\right)$. (Figura 9.7) 

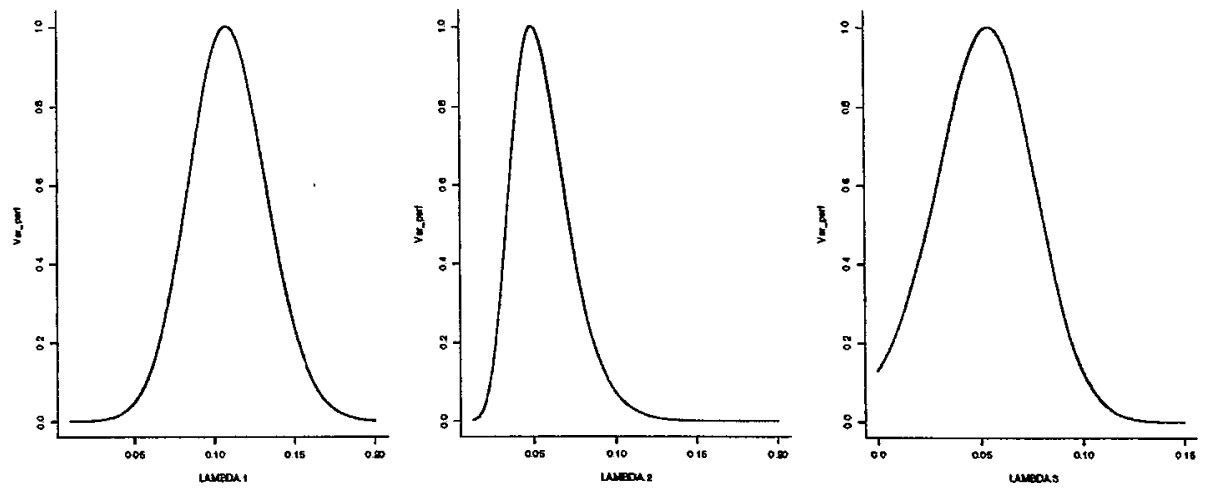

Figura 9.2: Gráfico da verossimilhança perfilada para $\lambda_{1}, \lambda_{2}$ e $\lambda_{3}$ respectivamente
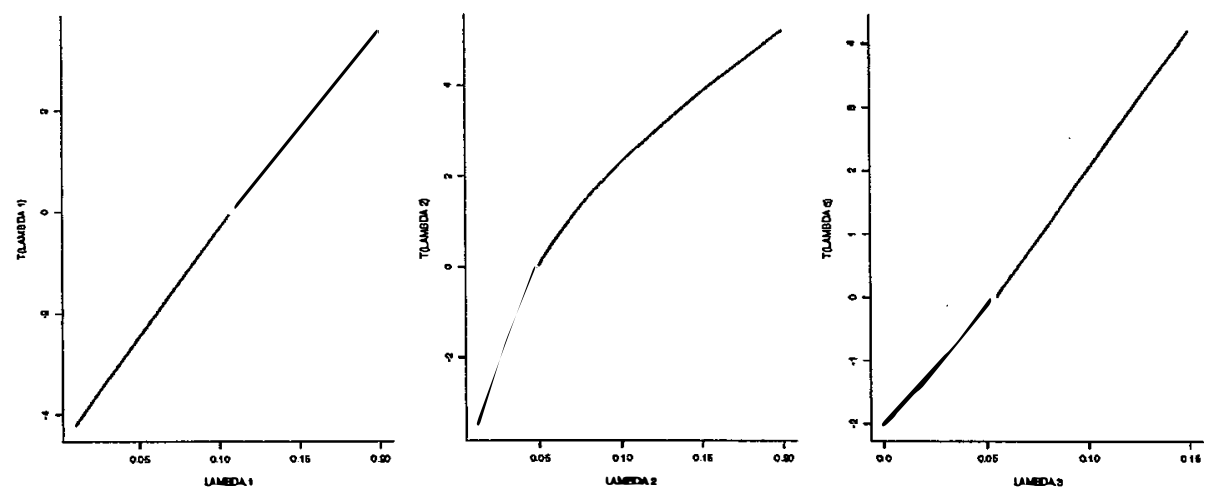

Figura 9.3: "t-plot" para $\lambda_{1}, \lambda_{2}$ e $\lambda_{3}$ respectivamente

Nas Figuras 9.5, 9.6 e 9.7 pode-se observar o quanto a reparametrização $\left(\theta_{1}, \theta_{2}, \theta_{3}\right)$ é ruim. Os gráficos dos "t-plots" apresentam uma não linearidade para todos os parâmetros $\theta_{1}, \theta_{2}, \theta_{3}$ mostrando assim que os gráficos das verossimilhançás perfiladas para os parâmetros $\theta_{1}, \theta_{2}, \theta_{3}$ estão "distantes" da distribuição normal. A "não proximidade da normal" pode ser observada também através dos gráficos de contorno apresentados na Figura 9.7 onde se podem observar claramente contornos não elípticos para a verossimilhança perfilada para os parâmetros $\left(\theta_{2}, \theta_{3}\right)$ e elipses não concêntricas para as verossimilhanças perfiladas $\left(\theta_{1}, \theta_{2}\right)$ e $\left(\theta_{1}, \theta_{3}\right)$. Outro aspecto a ser destacado também é a inversão de "inclinação" quando se comparam os gráficos das verossimilhanças perfiladas $\left(\lambda_{1}, \lambda_{3}\right)$ e $\left(\theta_{1}, \theta_{3}\right)$. 

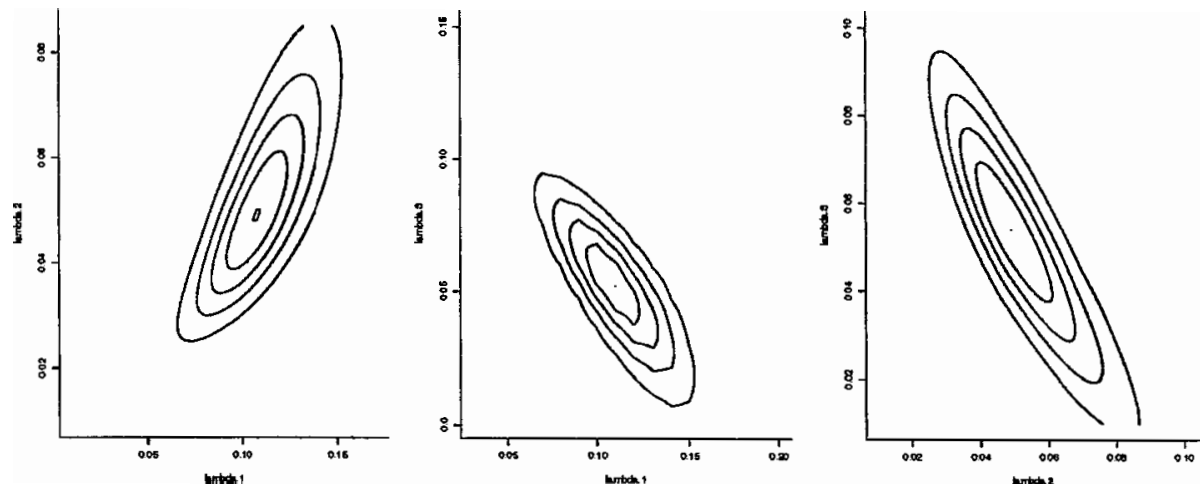

Figura 9.4: Contorno da verossimilhança perfilada para $\left(\lambda_{1}, \lambda_{2}\right),\left(\lambda_{1}, \lambda_{3}\right),\left(\lambda_{2}, \lambda_{3}\right)$ respectivamente
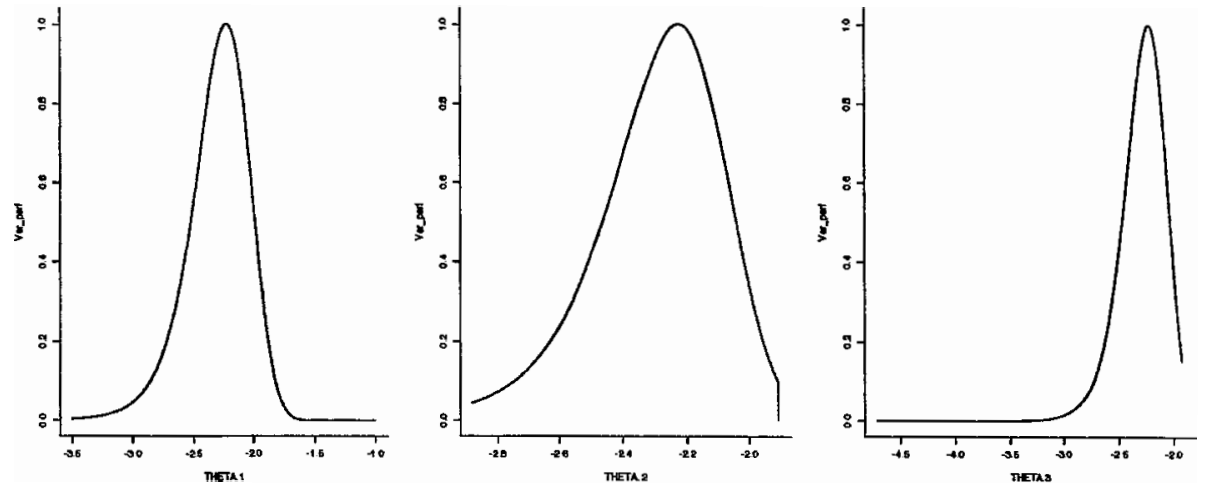

Figura 9.5: Gráfico da verossimilhança perfilada para $\theta_{1}, \theta_{2}$ e $\theta_{3}$ respectivamente 

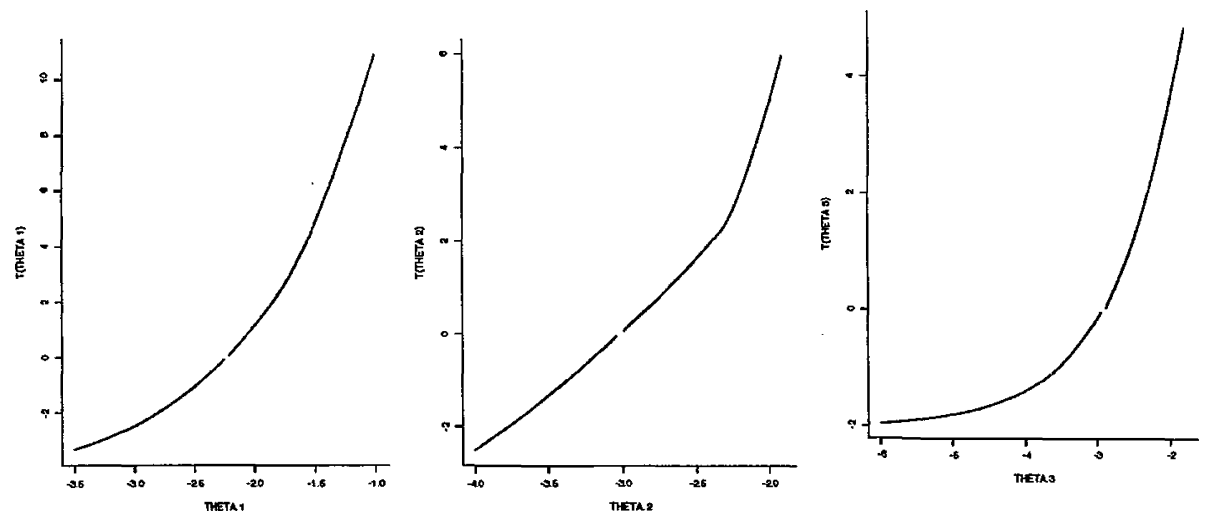

Figura 9.6: "t-plot" para $\theta_{1}, \theta_{2}$ e $\theta_{3}$ respectivamente
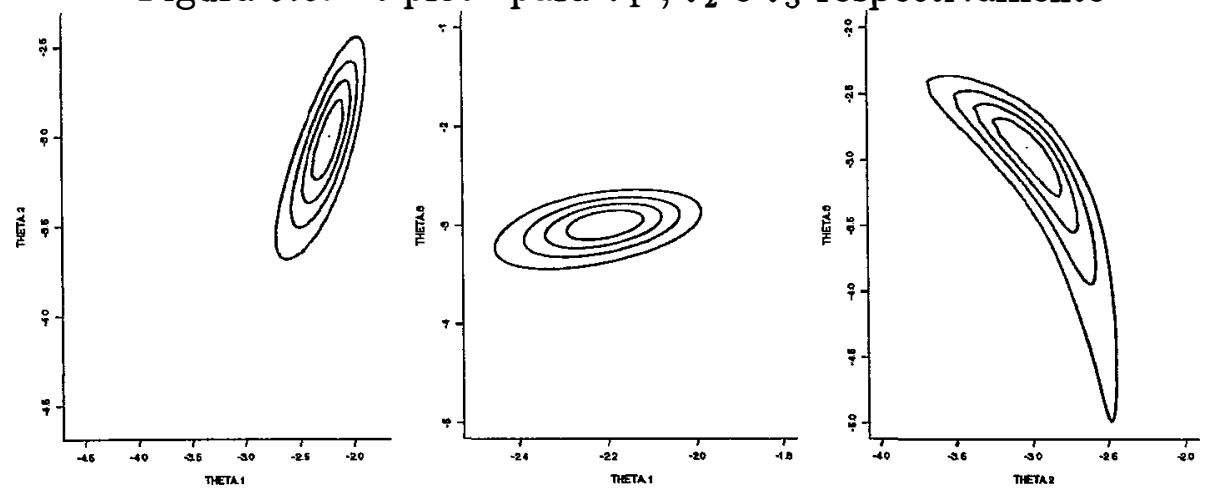

Figura 9.7: Contorno da verossimilhança perfilada para $\left(\theta_{1}, \theta_{2}\right),\left(\theta_{1}, \theta_{3}\right),\left(\theta_{2}, \theta_{3}\right)$ respectivamente

Considerando-se os dados de tempos de vida bivariados da Tabela 9.1 e a função de verossimilhança na parametrização $\left(\phi_{1}, \phi_{2}, \phi_{3}\right)$, foram construídos:

(a) Os gráficos da verossimilhança perfilada para os parâmetros: $\phi_{1}, \phi_{2}$ e $\phi_{3}$. (Figura 9.9).

(b) 0 "t-plot" para os parâmetros: $\phi_{1}, \phi_{2}$ e $\phi_{3}$. (Figura 9.9)

(c) Os contornos da verossimilhança perfilada para os parâmetros: $\left(\phi_{1}, \phi_{2}\right)$, $\left(\phi_{1}, \phi_{3}\right)$ e $\left(\phi_{2}, \phi_{3}\right)$. (Figura 9.10) 

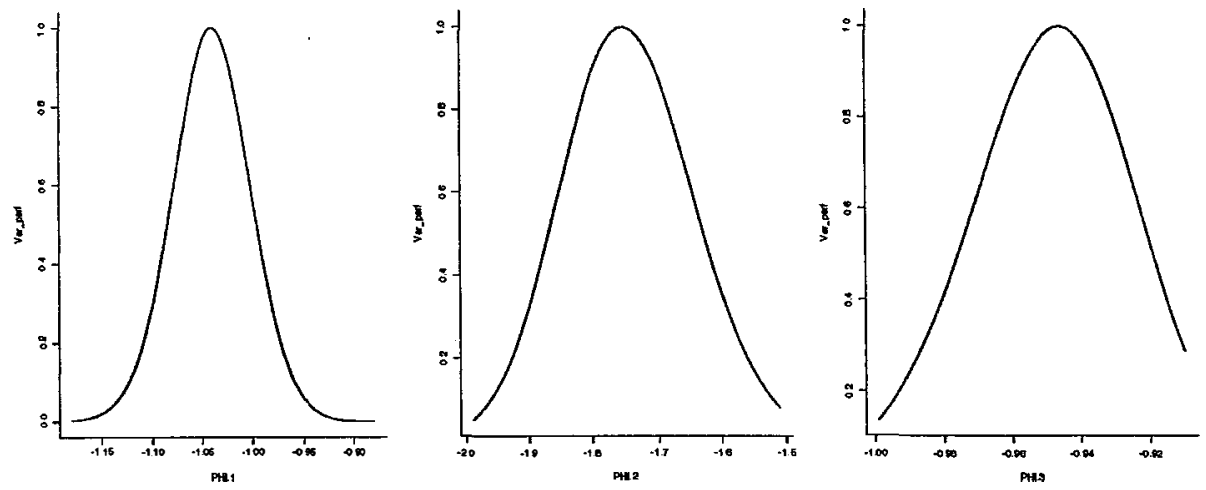

Figura 9.8: Gráfico da verossimilhança perfilada para $\phi_{1}, \phi_{2}$ e $\phi_{3}$ respectivamente
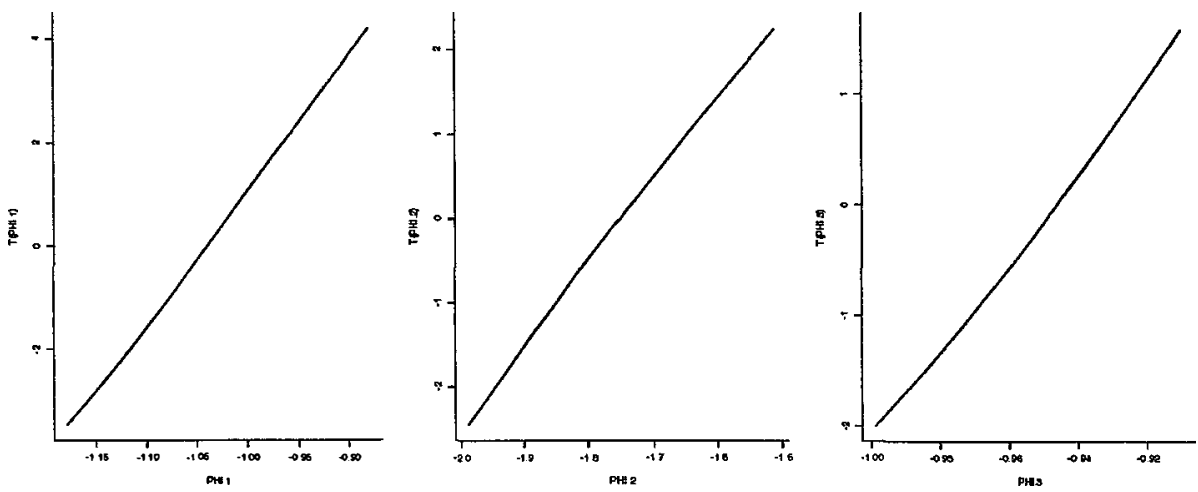

Figura 9.9: "t-plot" para $\phi_{1}, \phi_{2}$ e $\phi_{3}$ respectivamente 

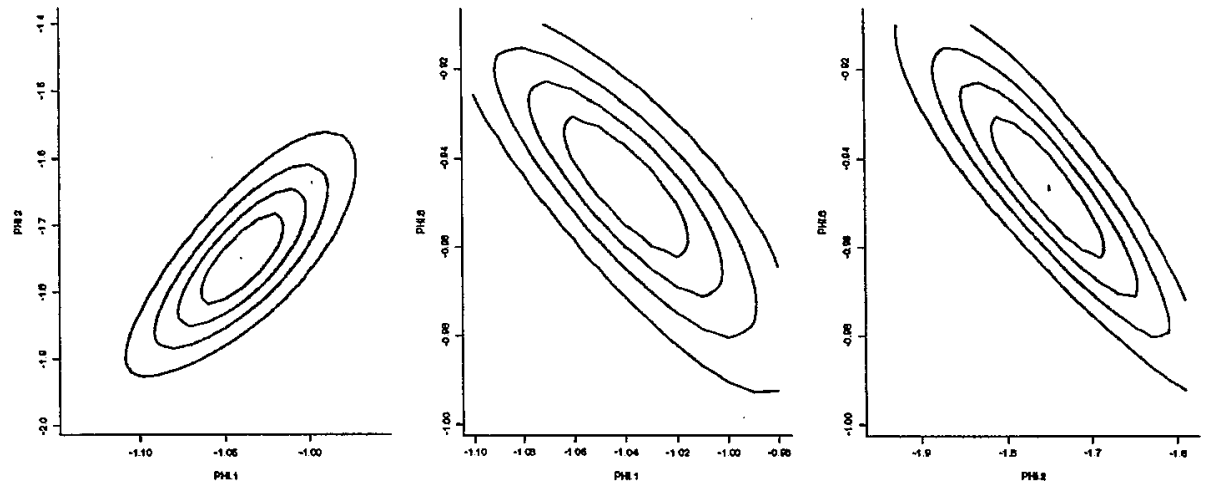

Figura 9.10: Contorno da verossimilhança perfilada para $\left(\phi_{1}, \phi_{2}\right),\left(\phi_{1}, \phi_{3}\right),\left(\phi_{2}, \phi_{3}\right)$ respectivamente

Através da Figura 9.9 pode ser observado o aspecto linear do "tplots" para todos os parâmetros $\phi_{1}, \phi_{2}, \phi_{3}$ indicando que as verossimilhanças perfiladas para os parâmetros $\phi_{1}, \phi_{2}, \phi_{3}$ estão "próximas da normalidade" considerando-se os gráficos de contorno da Figura 9.10 verifica o aspecto elíptico das verossimilhanças perfiladas para $\left(\phi_{1}, \phi_{2}\right),\left(\phi_{1}, \phi_{3}\right)$ e $\left(\phi_{2}, \phi_{3}\right)$

Através de métodos gráficos verifica-se que considerando-se as reparametrizações apresentadas neste trabalho a "mais" adequada é obtida quando é utilizada a reparametrização de Box \& Cox. Isso está de acordo com as conclusões obtidas através da utilização da medida de não normalidade conjunta proposta por KASS \& SLATE.

Na Tabela 9.4 apresentam-se os valores de STD, a confiabilidade do sistema em série para diferentes valores de $t_{0}, \hat{R}\left(t_{0}\right)=\hat{\theta}_{0}$ e o respectivo intervalo de confiança $95 \%$ para $\theta_{0}$ quando se considera a reparametrização $\left(\lambda_{1}, \lambda_{2}, \theta_{0}\right)$ e os dados da Tabela 9.1.

Considerando-se os dados de tempos de vida bivariados da Tabela 9.1 e a função de verossimilhança na parametrização $\left(\lambda_{1}, \lambda_{2}, \theta_{0}\right)$ foram construfdos os gráficos da verossimimilhança perfilada para $\theta_{0}$ com o respectivo "t-plot" para $t_{0}=1,2,3,5,10,20,30$. (Figuras 9.11,9.12) 
Tabela 9.4: Intervalos de Confiança $95 \%$ para $\theta_{0}$

\begin{tabular}{lllc}
\hline$t_{0}$ & $\hat{\theta_{0}}$ & STD & Intervalo de confiança $95 \%$ \\
\hline 1 & 0,8100 & 0,7236 & $(0,7780 ; 0,8420)$ \\
2 & 0,6561 & 0,6602 & $(0,6042 ; 0,7079)$ \\
3 & 0,5314 & 0,6066 & $(0,4684 ; 0,5944)$ \\
5 & 0,3486 & 0,5288 & $(0,2797 ; 0,4176)$ \\
10 & 0,1216 & 0,5069 & $(0,0735 ; 0,1696)$ \\
20 & 0,0148 & 1,2021 & $(0,0031 ; 0,0264)$ \\
30 & 0,0018 & 2,8827 & $(-0,0003 ; 0,0039)$ \\
\hline
\end{tabular}
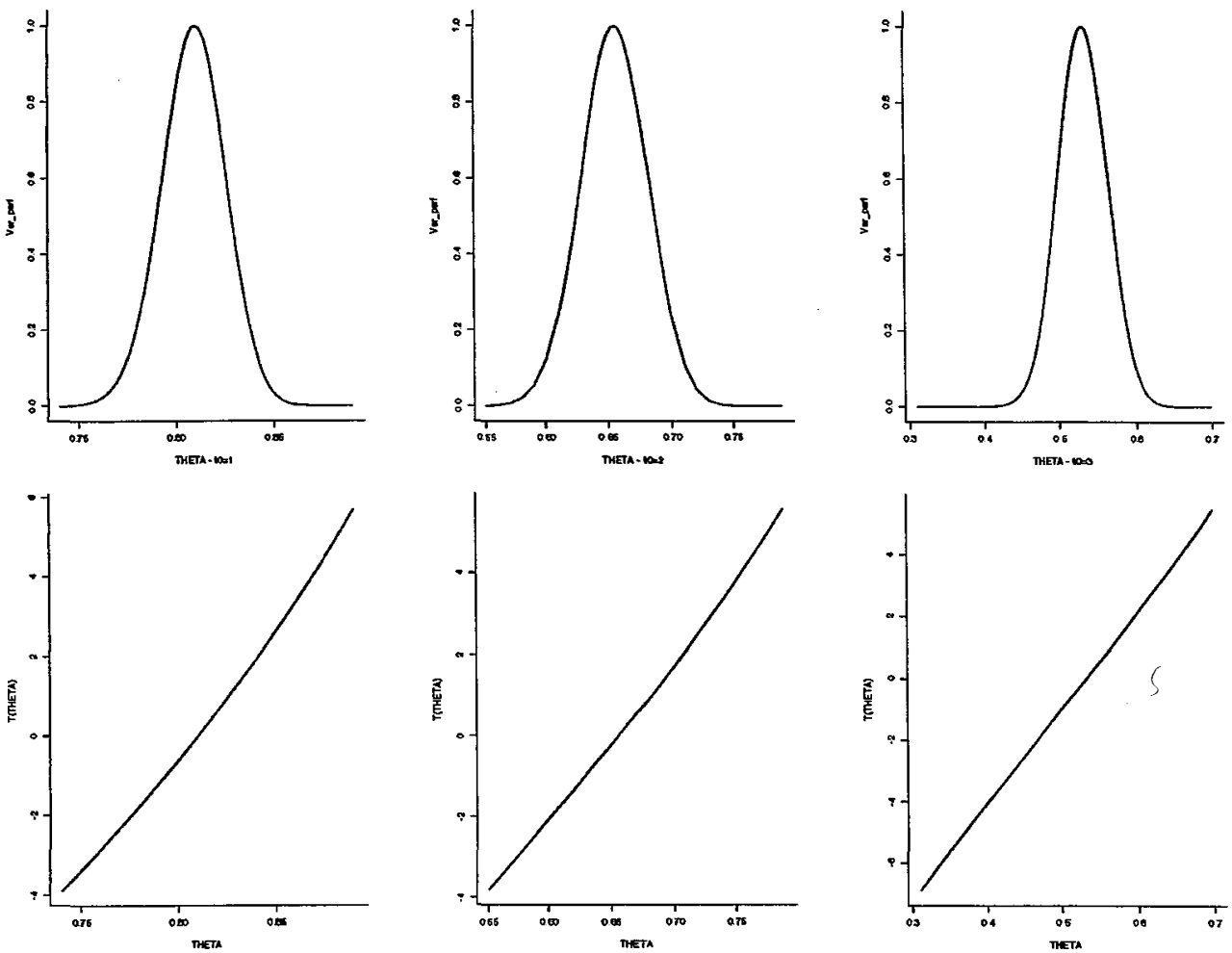

Figura 9.11: Gráfico da verossimilhança perfilada para $\theta_{0}$ com o respectivo "t-plot" nos tempos $t_{0}=1,2,3$ 

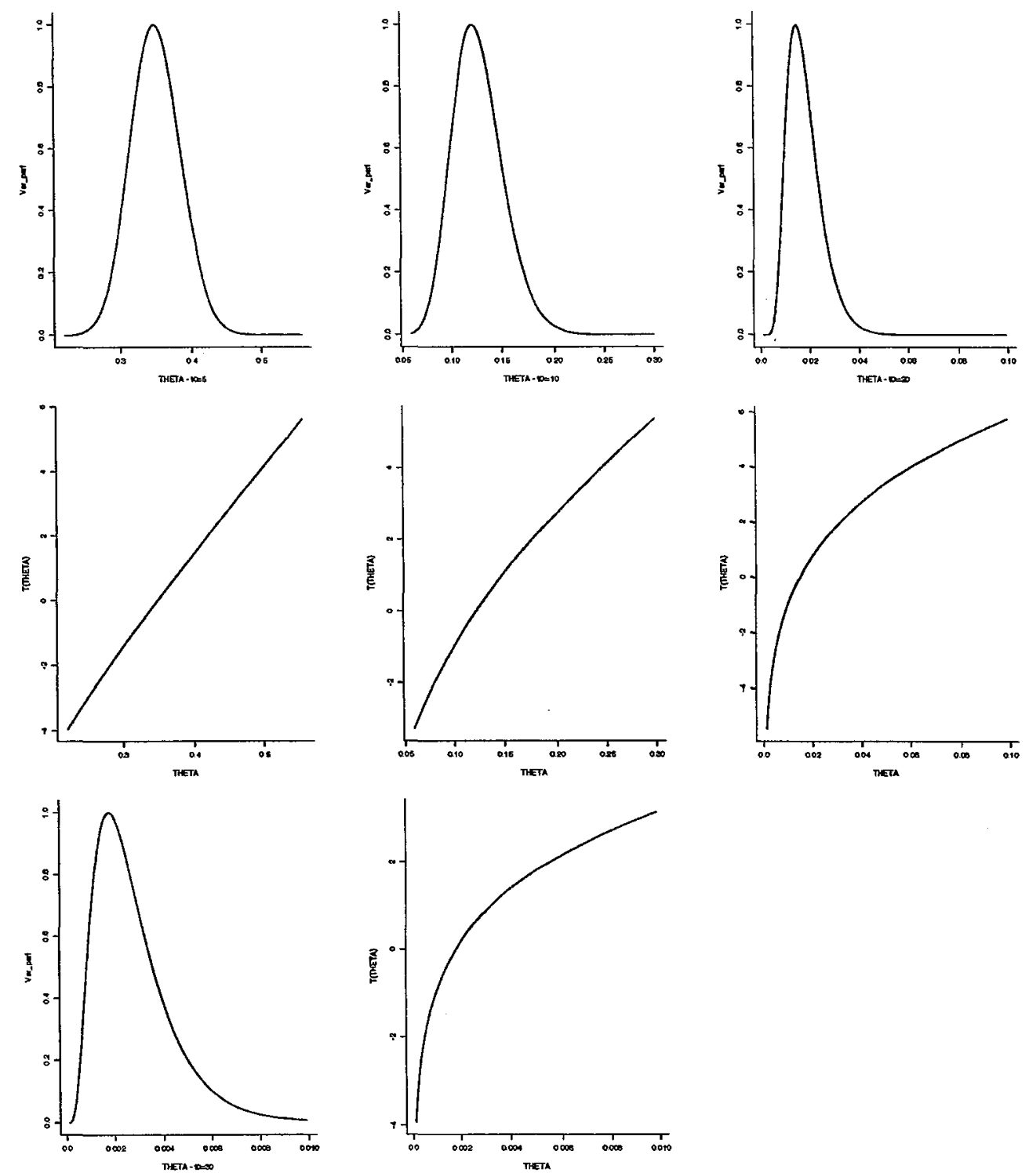

Figura 9.12: Gráfico da verossimilhança perfilada para $\theta_{0}$ com o respectivo "t-plot" nos tempos $t_{0}=5,10,20,30$ 
Tabela 9.5: Intervalo de confiança $95 \%$ para $\theta_{0}$

\begin{tabular}{llllc}
\hline$t_{0}$ & $\xi$ & $\hat{\theta}$ & STD & Intervalo de confiança $95 \%$ \\
\hline 1 & 0,6 & 0,8100 & 0,4875 & $(0,7734 ; 0,8383)$ \\
2 & 0,8 & 0,6561 & 0,4874 & $(0,5967 ; 0,7020)$ \\
3 & 0,8 & 0,5314 & 0,4878 & $(0,4608 ; 0,5881)$ \\
5 & 0,6 & 0,3486 & 0,4883 & $(0,2769 ; 0,4143)$ \\
10 & 0,6 & 0,1216 & 0,4865 & $(0,0757 ; 0,1709)$ \\
20 & 0,4 & 0,0148 & 0,4872 & $(0,0057 ; 0,0292)$ \\
30 & 0,2 & 0,0018 & 0,5035 & $(0,0005 ; 0,0052)$ \\
\hline
\end{tabular}

Na Tabela 9.5 apresentam-se os valores de STD, a confiabilidade do sistema em série para diferentes valores de $t_{0}, \hat{R}\left(t_{0}\right)=\hat{\theta_{0}}$ e o respectivo intervalo de confiança $95 \%$ para $\theta_{0}$ quando se considera a reparametrização $\left(\lambda_{1}, \lambda_{2}, \theta_{0}\right)$ e a transformação proposta por GuERRERo \& Johnson para $\theta_{0}$, observando-se que para cada valor de $t_{0}$ foi encontrado um valor para $\xi$ que conduziu ao menor valor de STD.

Considerando-se os dados de tempos de vida bivariados da Tabela 9.1 e a função de verossimilhança na parametrização $\left(\lambda_{1}, \lambda_{2}, \phi_{G J}\right)$ foram construídos os gráficos da verossimimilhança perfilada para $\phi_{G J}$ com o respectivo "tplot" para $t_{0}=1,2,3,5,10,20,30$. (Figuras 9.13, 9.14)

Comparando-se os resultados apresentados nas Tabelas 9.4 e 9.5 com a análise gráfica apresentada nas figuras $9.11,9.12,9.13$ e 9.14 podem-se observar mudanças no valor de STD e nos "t-plots", sendo que, uma mudança mais sensivel é observada para os valores de $t_{0}=20,30$, isto é, considerando-se a reparametrização de GuERRERo \& Johnson para a função de confiabilidade verifica-se uma melhora no valor da normalidade conjunta, STD da verossimimilhança na 
Tabela 9.6: Intervalo de confiança $95 \%$ para $\hat{\theta_{0}}$

\begin{tabular}{cllc}
\hline$t_{0}$ & $\theta$ & STD & \multicolumn{2}{c}{ Intervalo de confiança $95 \%$} \\
\hline 1 & 0,8100 & 18,8247 & $(0,7780 ; 0,8419)$ \\
2 & 0,6561 & 18,4667 & $(0,6043 ; 0,7078)$ \\
3 & 0,5314 & 18,1176 & $(0,4685 ; 0,5983)$ \\
5 & 0,3486 & 17,4460 & $(0,2798 ; 0,4175)$ \\
10 & 0,1216 & 15,9231 & $(0,0735 ; 0,1696)$ \\
20 & 0,0148 & 13,5542 & $(0,0031 ; 0,0263)$ \\
30 & 0,0018 & 12,1024 & $(-0,0003 ; 0,0039)$ \\
\hline
\end{tabular}

parametrização $\left(\lambda_{1}, \lambda_{2}, \phi_{G J}\right)$ e graficamente via "t-plot" pode-se verificar que para valores grandes de $t_{0}$ a melhora foi sensível.

Expressando-se $\lambda_{1}, \lambda_{2}$ como função $\left(\mu_{1}, \mu_{2}, \theta_{0}\right)$ pode-se incorporar a informação do especialista ao sistema inferencial.

Na Tabela 9.6 apresentam-se os valores de STD, a confiabilidade do sistema em série para diferentes valores de $t_{0}, \hat{R}\left(t_{0}\right)=\hat{\theta}_{0}$ e o respectivo intervalo de confiança $95 \%$ para $\theta_{0}$ quando se considera a reparametrização $\left(\mu_{1}, \mu_{2}, \theta_{0}\right)$ e os dados da Tabela 9.1.

Na Tabela 9.7 apresentam-se os valores de STD, a confiabilidade do sistema em série para diferentes valores de $t_{0}, \hat{R}\left(t_{0}\right)=\hat{\theta}_{0}$ e o respectivo intervalo de confiança $95 \%$ para $\theta_{0}$ quando se considera $\lambda_{1}, \lambda_{2}$ como função de $\mu_{1}, \mu_{2}$ e de $\theta_{0}$ e a transformação proposta por GUERRERo \& Johnson para $\theta_{0}$. Observando-se que para cada valor de $t_{0}$ foi encontrado um valor para $\xi$ que conduziu ao menor valor de STD.

Pode-se observar que os resultados obtidos para os intervalos de 
Tabela 9.7: Intervalo de Confiança $95 \%$ para $\theta_{0}$

\begin{tabular}{llllc}
\hline$t_{0}$ & $\xi$ & $\theta$ & STD & Intervalo de confiança $95 \%$ \\
\hline 1 & 1,0 & 0,8100 & 0,7985 & $(0,7715 ; 0,8374)$ \\
2 & 1,0 & 0,6561 & 0,7962 & $(0,5950 ; 0,7011)$ \\
3 & 1,0 & 0,5314 & 0,7955 & $(0,4586 ; 0,5870)$ \\
5 & 0,3 & 0,3486 & 0,9474 & $(0,2803 ; 0,4171)$ \\
10 & 0,3 & 0,1216 & 0,9570 & $(0,0787 ; 0,1742)$ \\
20 & 0,6 & 0,0148 & 0,8040 & $(0,0050 ; 0,0281)$ \\
30 & 0,6 & 0,0018 & 0,7995 & $(0,0002 ; 0,0044)$ \\
\hline
\end{tabular}

confiança $95 \%$ para $\theta_{0}$ obtidos nas Tabela 9.4 coincidem com os resultados apresentados na Tabela 9.6. A menos de arredondamento isto significa que a nova maneira de expressar $\lambda_{1}$ e $\lambda_{2}$ não tem influência na normalidade do parâmetro $\theta_{0}$ embora exerça uma forte influência na normalidade conjunta da função de verossimilhança. Quando se considera a reparametrização de GuERRERo \& JOHNSON para a função de confiabilidade verifica-se uma melhora no valor da normalidade conjunta, STD da verossimimilhança na parametrização $\left(\mu_{1}, \mu_{2}, \phi_{G J}\right)$ (Tabela 9.8) comparado com o valor de STD da verossimimilhança na parametrização $\left(\mu_{1}, \mu_{2}, \theta_{0}\right)$ (Tabela 9.7).

Concluindo, quando se consideram parametrizações que incluem a função de confiabilidade em ambos os casos observa-se que transformação de GUERRERO \& JOHNSON produz os melhores resultados para STD.

É necessário implentar um programa computacional distinto para a construção de cada uma das verossimilhanças perfiladas.

Deve-se observar que o método iterativo de Newton-Raphson (ver por exemplo, LEE, 1992) é utilizado somente quando se usa a parametrização 

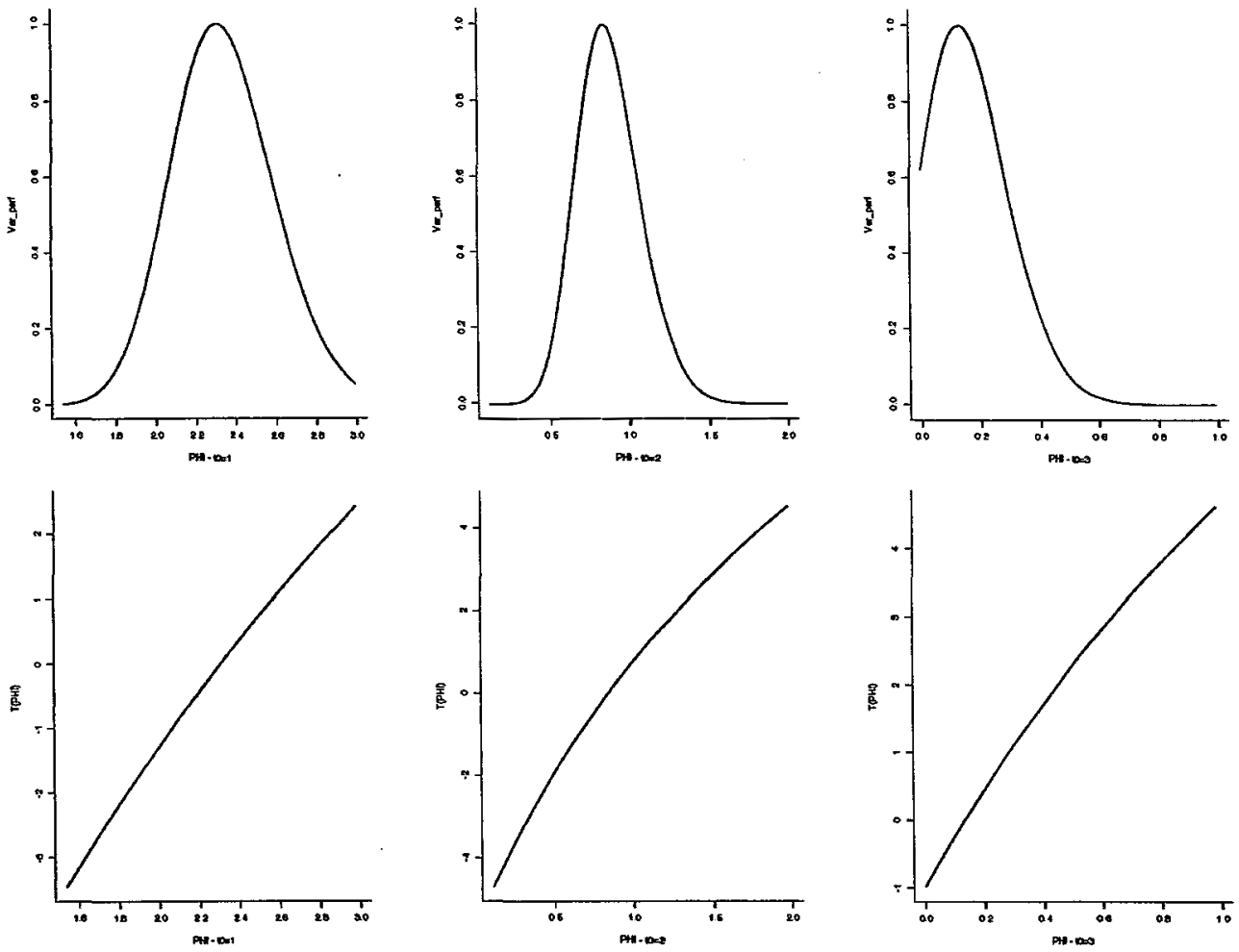

Figura 9.13: Gráfico da verossimilhança perfilada para $\phi_{G J}$ com o respectivo "t-plot" nos tempos $t_{0}=1,2,3$

$\left(\lambda_{1}, \lambda_{2}, \lambda_{3}\right)$ e a reparametrização $\left(\theta_{1}, \theta_{2}, \theta_{3}\right)$. Nas outras reparametrizações utiliza-se um método de otimização não linear no qual as derivadas de primeira e segunda ordem são calculadas por diferenças finitas. Esta mudança foi necessária porque na implementação do método de Newton-Raphson é necessário resolver um sistema de equações lineares que pode ser singular ou mal-condicionado dadas as expressões complicadas das derivadas analíticas de primeira e segunda ordem obtidas com estas reparametrizações. 

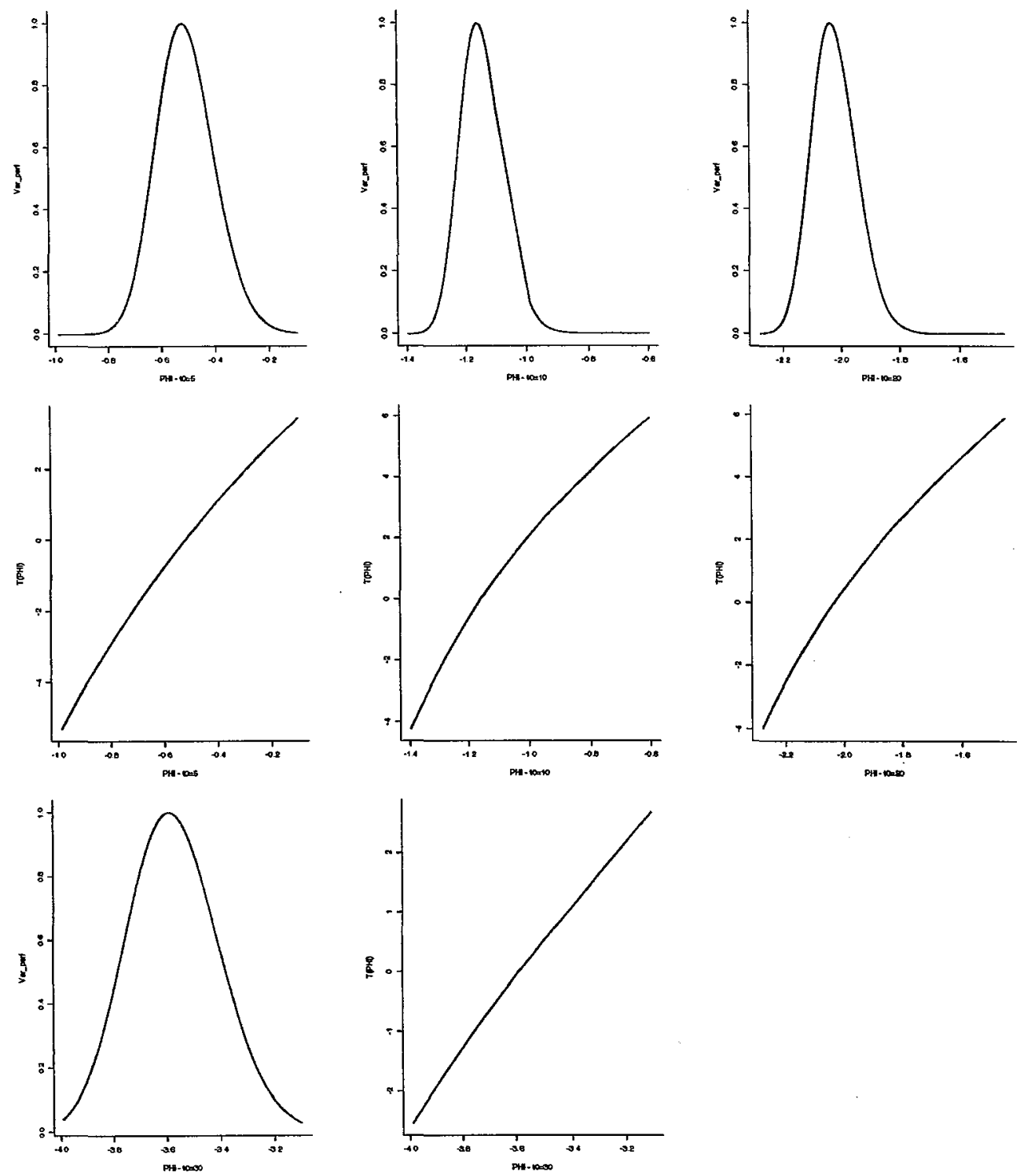

Figura 9.14: Gráfico da verossimilhança perfilada para $\phi_{G J}$ com o respectivo "t-plot" nos tempos $t_{0}=5,10,20,30$ 


\subsection{Exemplo 02}

Neste exemplo desenvolve-se a análise Bayesiana apresentada no Capítulo 6 para o modelo de BLOCK \& BASU utilizando-se o algoritmo "Gibbs-comMetropolis-Hastings" (G-MH) considerando-se dois conjuntos de dados de tempo de vida bivariados: os dados da Tabela 9.8 apresentados por SANTANDER (1993) e os dados da Tabela 9.1 gerados por Leandro \& AchCAR (1995). Realiza-se, também, um estudo comparativo entre os resultados obtidos por SANTANDER (1993) utilizando o método de aproximação de Laplace e o resultado obtido através do algoritmo G-MH para o conjunto de dados I.

\subsubsection{Análise do conjunto de dados I}

Na Tabela 9.8, têm-se 30 observações bivariadas $(X, Y)$ geradas, considerando-se o modelo ACBVE (2.1) com parâmetros $\lambda_{1}=0,25, \lambda_{2}=0,16 \mathrm{e}$ $\lambda_{3}=0,0$ dados em SAntander \& AChCAR (1993). Deve-se observar que como $\lambda_{3}=0,0$ então as funções densidades de probabilidade marginais para $X$ e $Y$ são exponencialmente distribuidas.

Para inferência Bayesiana assumem-se as densidades a priori para $N_{1} \sim b\left(30, \lambda_{1} / \lambda_{12}\right), \lambda_{1} \sim \Gamma\left(a_{1}, b_{1}\right), \lambda_{2} \sim \Gamma\left(a_{2}, b_{2}\right)$ e $\lambda_{3} \sim \Gamma\left(a_{3}, b_{3}\right)$ dadas em (6.7) onde $a_{1}=115, b_{1}=480, a_{2}=56, a_{3}=20$ e $b_{3}=1400$ e as distribuições $a$ posteriori condicionais cheias dadas pela expressão (6.10) com $r=16, n-r=14, n=30$, $n \bar{x}=114,51, n \bar{y}=165,67$ e $R m=207,77$. Para se obter uma amostra de Gibbs através do algoritmo G-MH, procede-se da seguinte forma: geram-se $m=10$ cadeias (ou réplicas) de tamanho $2 p=2000$, utilizando-se 10 pontos iniciais distintos para $N_{1}, \lambda_{1}, \lambda_{2}, \lambda_{3}$. Desprezam-se as $p=1000$ primeiras iterações, com o objetivo de diminuir o efeito dos pontos iniciais, e a partir daí escolhem-se as iterações de 10 
Tabela 9.8: Dados de tempo de vida bivariados gerados $\operatorname{com} \lambda_{1}=0,25, \lambda_{2}=0,16$ e $\lambda_{3}=0,0$

\begin{tabular}{rrrrrr}
\hline$i$ & $x$ & $y$ & $i$ & $x$ & $y$ \\
\hline 1 & 3,73 & 2,54 & 16 & 3,42 & 1,09 \\
2 & 5,83 & 7,74 & 17 & 7,71 & 0,33 \\
3 & 8,44 & 9,89 & 18 & 6,92 & 2,59 \\
4 & 7,95 & 2,47 & 19 & 7,76 & 3,77 \\
5 & 7,66 & 8,77 & 20 & 0,16 & 6,07 \\
6 & 3,47 & 1,86 & 21 & 7,79 & 6,98 \\
7 & 2,75 & 1,30 & 22 & 0,66 & 0,49 \\
8 & 0,57 & 5,04 & 23 & 10,83 & 4,03 \\
9 & 3,48 & 1,13 & 24 & 4,23 & 2,71 \\
10 & 4,12 & 7,24 & 25 & 3,23 & 18,74 \\
11 & 2,08 & 9,40 & 26 & 1,00 & 9,10 \\
12 & 4,19 & 1,50 & 27 & 3,08 & 12,43 \\
13 & 0,82 & 6,29 & 28 & 0,55 & 13,50 \\
14 & 1,14 & 2,61 & 29 & 0,37 & 5,52 \\
15 & 0,18 & 8,17 & 30 & 0,39 & 2,37 \\
\hline & & & & &
\end{tabular}

em 10, ou seja, para cada parâmetro considera-se a 1010-ésima, 1020-ésima, ..., 2000-ésima iteração ; daí para 10 cadeias fornecem uma amostra de tamanho 1000. Para monitorar a convergência utiliza-se o método de Gelman \& Rubin (1992) que utiliza a técnica da análise da variância para determinar se um maior número de iterações é necessário.

Na Tabela 9.9 apresenta-se um resumo da distribuição a posteriori marginal aproximada obtida e o resultado do monitoramento da convergência.

Como pode ser observado na Tabela 9.9 o valor de $\hat{R}$ está próximo de 1 para todos os parâmetros, portanto, segundo GeLman \& RUBin a convergência foi obtida e a amostra considerada fornece um sumário apropriado para os parâme$\operatorname{tros} \lambda_{1}, \lambda_{2}, \lambda_{3}$.

Na Tabela 9.10 apresentam-se os intervalos de confiança e de credi- 
Tabela 9.9: Estatísticas da distribuição a posteriori marginal aproximada via G$\mathrm{MH}$ e monitoramento da convergência

\begin{tabular}{lccc}
\hline Parâmetro & $\lambda_{1}$ & $\lambda_{2}$ & $\lambda_{3}$ \\
Mínimo & 0,1841 & 0,1240 & 0,0072 \\
1o. Quartil & 0,2371 & 0,1536 & 0,0137 \\
Mediana & 0,2517 & 0,1646 & 0,0161 \\
Média & 0,2527 & 0,1651 & 0,0163 \\
3o. Quartil & 0,2681 & 0,1757 & 0,0184 \\
Máximo & 0,3128 & 0,2499 & 0,0282 \\
Desvio-Padrão & 0,0223 & 0,0168 & 0,0036 \\
\hline \multicolumn{4}{c}{ Monitoramento da Convergência } \\
\hline$\hat{R}$ & 1,0593 & 1,0424 & 1,01088 \\
\hline
\end{tabular}

bilidade a $95 \%$ obtidos por ACHCAR \& SANTANDER (1993) e o obtido utilizando-se o algoritmo de Gibbs-com-Metropolis-Hastings (G-MH).

Como se pode observar, os intervalos de credibilidade quando foi utilizada a priori informativa e o algoritmo de G-MH estão bastantes próximos.

Na Tabela 9.11 apresentam-se as estimativas dos tempos de vida médio obtidos por ACHCAR \& SANTANDER (1993) e pelo algoritmo de G-MH.

Pode-se verificar através da Tabela 9.11 resultados próximos para as estimativas de tempo de vida médios obtidos pelo método de aproximação de Laplace com a densidade a priori informativa e os resultados obtidos pelo algoritmo G-MH.

Na tabela 9.12 apresentam-se as estimativas da função de con- 
Tabela 9.10: Intervalos de confiança e de credibilidade a $95 \%$

\begin{tabular}{lccc}
\hline Parâmetro & $\lambda_{1}$ & $\lambda_{2}$ & $\lambda_{3}$ \\
EMV & 0,2485 & 0,1698 & 0,0164 \\
I. Confiança 95\% & $(0,0710 ; 0,4260)$ & $(0,0090 ; 0,3310)$ & $(-0,1830 ; 0,2160)$ \\
\hline I. Credibilidade 95\% & & & \\
Priori não-Informativa & $(0,0700 ; 0,4360)$ & $(0,0500 ; 0,3360)$ & $(-0,1820 ; 0,2100)$ \\
Priori Informativa & $(0,2120 ; 0,2920)$ & $(0,1330 ; 0,1990)$ & $(0,0060 ; 0,0440)$ \\
\hline G-MH & $(0,2051 ; 0,2942)$ & $(0,1342 ; 0,2010)$ & $(0,0098 ; 0,0249)$ \\
\hline
\end{tabular}

Tabela 9.11: Estimativas de tempo de vida médios

\begin{tabular}{cc|c|cc|c}
\hline & Valor real & EMV & \multicolumn{2}{|c|}{ Aproximação de Laplace } & G-MH \\
\hline & & & Priori não informativa & Priori Informativa & \\
\hline$\mu_{1}$ & 4,00 & 3,8322 & 3,7981 & 3,8057 & 3,8009 \\
& 6,25 & 5,4907 & 6,2073 & 5,7001 & 5,6903 \\
\hline
\end{tabular}

fiabilidade para um sistema em série e em paralelo no tempo $t_{0}, R_{\mathcal{S}}\left(t_{0}\right)$ e $R_{P}\left(t_{0}\right)$ respectivamente, obtidos por ACHCAR \& SANTANDER (1993) e pelo algoritmo GMH.

Na Figura 9.15 apresenta-se o gráfico da densidade a posteriori marginal aproximada para $\lambda_{1}, \lambda_{2}$ e $\lambda_{3}$, respectivamente. 
Tabela 9.12: Estimativas de Bayes para a confiabilidade

\begin{tabular}{lcccccccc}
\cline { 3 - 8 } tempo & \multicolumn{2}{c}{ EMV } & \multicolumn{3}{c}{ Aproximação de Laplace } & \multicolumn{2}{c}{ G-MH } \\
\hline \multicolumn{1}{c}{$R_{S}\left(t_{0}\right)$} & $R_{P}\left(t_{0}\right)$ & $R_{S}\left(t_{0}\right)$ & $R_{P}\left(t_{0}\right)$ & $R_{S}\left(t_{0}\right)$ & $R_{P}\left(t_{0}\right)$ & $R_{S}\left(t_{0}\right)$ & $R_{P}\left(t_{0}\right)$ \\
\hline 1 & 0,6636 & 0,9673 & 0,6610 & 0,9657 & 0,6499 & 0,9624 & 0,6481 & 0,9622 \\
2 & 0,4404 & 0,8922 & 0,4369 & 0,8874 & 0,4226 & 0,8780 & 0,4204 & 0,8774 \\
3 & 0,2923 & 0,7989 & 0,2888 & 0,7907 & 0,2751 & 0,7748 & 0,2730 & 0,7742 \\
4 & 0,1940 & 0,7012 & 0,1909 & 0,6903 & 0,1792 & 0,6694 & 0,1774 & 0,6686 \\
5 & 0,1287 & 0,6071 & 0,1262 & 0,5943 & 0,1168 & 0,5699 & 0,1154 & 0,5691 \\
10 & 0,0166 & 0,2674 & 0,0159 & 0,2543 & 0,0139 & 0,2299 & 0,0136 & 0,2296 \\
15 & 0,0021 & 0,1121 & 0,0020 & 0,1038 & 0,0017 & 0,0884 & 0,0016 & 0,0884 \\
\hline
\end{tabular}
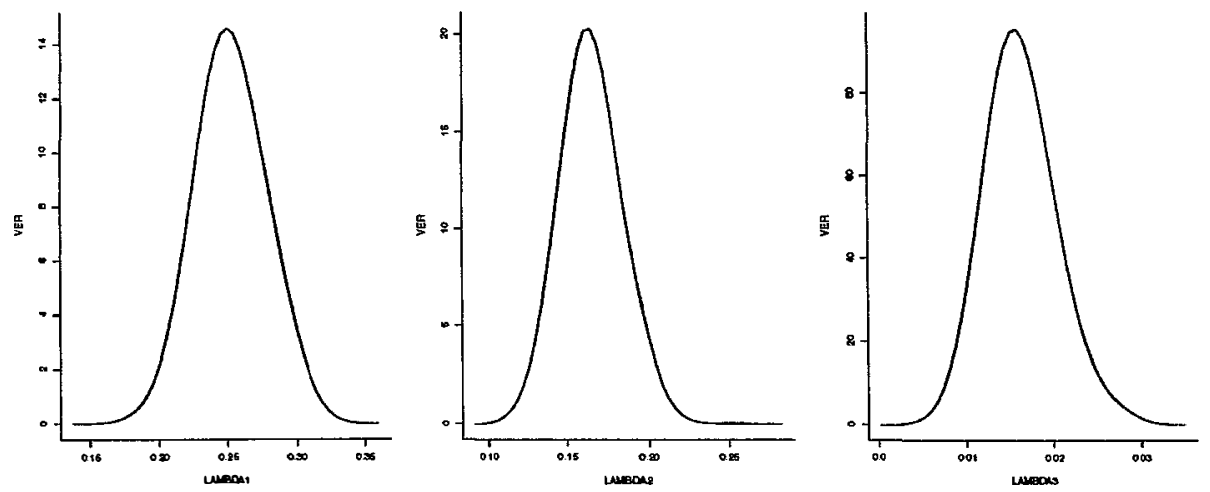

Figura 9.15: Gráficos das densidades a posteriori marginais aproximadas para $\lambda_{1}$, $\lambda_{2}, \lambda_{3}$ 


\subsubsection{Análise do conjunto de dados II}

Considerando-se os dados de tempo de vida bivariados gerados por LEANDRO \& ACHCAR (1995) apresentados na Tabela 9.1, as densidades a priori dadas pela expressão (6.7) onde $a_{1}=116, b_{1}=1078, a_{2}=240, b_{2}=4900, a_{3}=291 \mathrm{e}$ $b_{3}=5400 \mathrm{e}$ as densidades a posteriori condicionais cheias dadas pela expressão (6.10) $\operatorname{com} r=71, n-r=29, n=100, n \bar{x}=674,45, n \bar{y}=1118,88$ e $R m=1343,65$. Para se obter uma amostra de Gibbs através do algoritmo G-MH procede-se da seguinte forma: geram-se $m=10$ cadeias (ou réplicas) de tamanho $2 p=2000$, utilizando-se 10 pontos iniciais distintos para $N_{1}, \lambda_{1}, \lambda_{2}, \lambda_{3}$. Desprezam-se as $p=1000$ primeiras iterações, com o objetivo de diminuir o efeito dos pontos iniciais, e a partir daf escolhem-se as iterações de 10 em 10, ou seja, para cada parâmetro considera-se a 1010-ésima, 1020-ésima, ..., 2000-ésima iteração ; daí para 10 cadeias fornecem uma amostra de tamanho 1000. Para monitorar a convergência utiliza-se o método de Gelman \& Rubin (1992) que utiliza a técnica da análise da variância para determinar se um maior número de iterações é necessário.

Na Tabela 9.13 apresenta-se o resumo da distribuição a posteriori juntamente com o resultado do monitoramento da convergência da amostra Gibbs obtida utilizando o algoritmo de Gibbs-com-Metropolis-Hastings (G-MH). Na Tabela 9.14 apresenta-se o intervalo de confiança a $95 \%$ admitindo-se a normalidade assintótica dos estimadores de máxima verossimilhança e o intervalo de credibilidade a $95 \%$ obtido através da amostra G-MH.

Nas Tabelas 9.15 e 9.16 apresentam-se as estimativas para os tempos de vida médio e para a função de confiabilidade para o sistema com dois componentes em série e em paralelo no tempo $t_{0}$ considerando os dados gerados e 
Tabela 9.13: Estatísticas da distribuição a posteriori aproximada

\begin{tabular}{lccc}
\hline Parâmetro & $\lambda_{1}$ & $\lambda_{2}$ & $\lambda_{3}$ \\
Mínimo & 0,0797 & 0,0457 & 0,0498 \\
1o. Quartil & 0,0990 & 0,0477 & 0,0528 \\
Mediana & 0,1066 & 0,0486 & 0,0535 \\
Média & 0,1067 & 0,0486 & 0,0535 \\
3o. Quartil & 0,1137 & 0,0494 & 0,0563 \\
Máximo & 0,1333 & 0,0543 & 0,0563 \\
Desvio-Padrão & 0,0106 & 0,0010 & 0,0003 \\
\hline \multicolumn{4}{c}{ Monitoramento da Convergência } \\
\hline$\hat{R}$ & 1,0368 & 1,02734 & 1,0382 \\
\hline
\end{tabular}

os dados obtidos via G-MH.

Considerando-se a análise do conjunto de dados I verifica-se a proximidade dos resultados obtidos quando se utiliza o método de aproximação de Laplace com uma densidade a priori informativa e os resultados obtidos através do algoritmo G-MH. Considerando-se o conjunto de dados II verifica-se a proximidade entre os valores reais e os obtidos através da amostra G-MH para a confiabilidade.

Estes resultados mostram a importância da amostragem via G-MH que facilita bastante a análise e interpretação dos dados exigindo apenas o uso de estatística descritiva. 
Tabela 9.14: Intervalos de confiança e de credibilidade a $95 \%$

\begin{tabular}{clcc}
\hline & EMV & Intervalo de confiança $95 \%$ & Intervalo de Credibilidade G-MH \\
\hline$\lambda_{1}$ & 0,10782 & $(0,0605 ; 0,1551)$ & $(0,0875 ; 0,1333)$ \\
$\lambda_{2}$ & 0,04896 & $(0,0163 ; 0,0816)$ & $(0,0467 ; 0,0511)$ \\
$\lambda_{3}$ & 0,0164 & $(0,0072 ; 0,1007)$ & $(0,0514 ; 0,0557)$ \\
\hline
\end{tabular}

Tabela 9.15: Estimativas de tempo de vida médios

\begin{tabular}{cccc}
\hline & Valor real & EMV & G-MH \\
\hline$\mu_{1}$ & 8,0612 & 6,6755 & 6,7795 \\
$\mu_{2}$ & 9,7619 & 11,4272 & 11,5094 \\
\hline
\end{tabular}

Tabela 9.16: Estimativas de Bayes para a confiabilidade

\begin{tabular}{lcccccc}
\hline tempo & \multicolumn{2}{c}{ Valor Real } & \multicolumn{2}{c}{ EMV } & \multicolumn{2}{c}{ G-MH } \\
\hline & $R_{S}\left(t_{0}\right)$ & $R_{P}\left(t_{0}\right)$ & $R_{S}\left(t_{0}\right)$ & $R_{P}\left(t_{0}\right)$ & $R_{S}\left(t_{0}\right)$ & $R_{P}\left(t_{0}\right)$ \\
\hline 1 & 0,8187 & 0,9885 & 0,8100 & 0,9886 & 0,8115 & 0,9887 \\
2 & 0,6703 & 0,9586 & 0,6561 & 0,9590 & 0,6586 & 0,9596 \\
3 & 0,5488 & 0,9161 & 0,5314 & 0,9172 & 0,5346 & 0,9180 \\
4 & 0,4493 & 0,8655 & 0,4304 & 0,8676 & 0,4340 & 0,8694 \\
5 & 0,3679 & 0,8102 & 0,3486 & 0,8134 & 0,3523 & 0,8159 \\
10 & 0,1353 & 0,5312 & 0,1215 & 0,5416 & 0,1245 & 0,5463 \\
15 & 0,0498 & 0,3186 & 0,0424 & 0,3342 & 0,0441 & 0,3393 \\
\hline
\end{tabular}



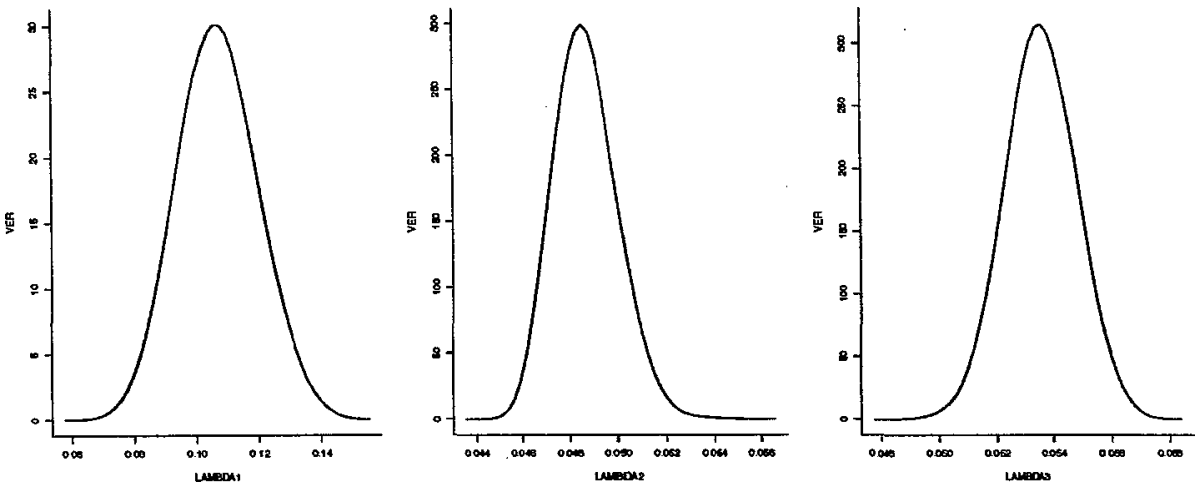

Figura 9.16: Gráficos das densidades a posteriori marginais aproximadas considerando-se $\lambda_{1}=0,08, \lambda_{2}=0,06, \lambda_{3}=0,06$. 


\subsection{Exemplo 03}

Neste exemplo desenvolve-se a análise Bayesiana do modelo ACBVE (2.1) em testes de vida acelerados com o modelo de potência utilizando-se o algoritmo de "Gibbs-com-Metroplis-Hastings" (G-MH) apresentada no Capítulo 7, considerando-se os dados de tempo de vida bivariados apresentados por SANTANDER (1993) $\operatorname{com} \mathcal{P}=2, V_{1}=1, V_{2}=2$ e $V_{3}=3$. A seguir faz-se um estudo comparativo entre os resultados obtidos via amostra gerada pelo algoritmo G-MH com os resultados obtidos para o mesmo problema por SANTANDER (1993). Os dados são apresentados na Tabela 9.17.

Tabela 9.17: Dados de tempo de vida bivariados gerados com aplicação de estresse

\begin{tabular}{ccc}
\hline$V_{1}=1$ & $V_{2}=2$ & $V_{3}=3$ \\
\hline$\left(X_{1 i}, Y_{1 i}\right)$ & $\left(X_{2 i}, Y_{2 i}\right)$ & $\left(X_{3 i}, Y_{3 i}\right)$ \\
\hline$(7,65 ; 2,18)$ & $(2,29 ; 0,02)$ & $(0,34 ; 0,20)$ \\
$(16,67 ; 9,26)$ & $(0,10 ; 0,38)$ & $(1,50 ; 1,30)$ \\
$(39,30 ; 6,72)$ & $(0,88 ; 0,27)$ & $(0,63 ; 0,69)$ \\
$(1,30 ; 3,22)$ & $(0,45 ; 0,04)$ & $(0,68 ; 0,12)$ \\
$(9,04 ; 2,23)$ & $(1,66 ; 1,60)$ & $(3,22 ; 0,09)$ \\
$(5,15 ; 0,41)$ & $(0,74 ; 1,67)$ & $(1,91 ; 0,91)$ \\
$(5,20 ; 5,91)$ & $(2,50 ; 0,37)$ & $(0,52 ; 0,58)$ \\
$(5,00 ; 0,84)$ & $(3,50 ; 0,03)$ & $(0,30 ; 0,01)$ \\
$(5,66 ; 0,42)$ & $(8,45 ; 0,71)$ & $(1,30 ; 0,02)$ \\
$(11,80 ; 0,15)$ & $(4,60 ; 0,83)$ & $(0,52 ; 0,10)$ \\
$(17,08 ; 10,37)$ & $(2,66 ; 1,06)$ & $(2,08 ; 0,30)$ \\
$(17,92 ; 0,76)$ & $(1,46 ; 1,04)$ & $(0,95 ; 0,91)$ \\
$(1,62 ; 2,73)$ & $(1,03 ; 0,41)$ & $(0,43 ; 0,02)$ \\
$(1,42 ; 1,85)$ & $(4,36 ; 1,34)$ & $(0,25 ; 0,08)$ \\
$(3,60 ; 1,50)$ & $(0,76 ; 0,77)$ & $(1,39 ; 0,08)$ \\
\hline
\end{tabular}

Para análise Bayesiana consideram-se as densidades a priori dadas por (7.15): $N_{1} \sim b\left(15, c_{1} / c_{12}\right), \quad c_{1} \sim \Gamma\left(a_{1}, b_{1}\right), \quad c_{2} \sim \Gamma\left(a_{2}, b_{2}\right), \quad c_{3} \sim \Gamma\left(a_{3}, b_{3}\right), \quad \mathrm{e}$ $\mathcal{P} \sim N\left(\mu_{0}, \sigma_{0}^{2}\right)$, onde $a_{1}=5, b_{1}=72, a_{2}=15, b_{2}=54, a_{3}=3, b_{3}=41, \mu_{0}=2 \mathrm{e}$ 
$\sigma_{0}^{2}=0,05$ e as densidades a posteriori condicionais cheias dadas por (7.17) usando-se os valores apresentados na Tabela 9.18.

Tabela 9.18: Valores auxiliares

\begin{tabular}{rrrrrrr}
\hline$j$ & $V_{j}$ & $r_{j}$ & $n_{j}$ & $n_{j} \bar{X}_{j}$ & $n_{j} \bar{Y}_{j}$ & $R_{j}$ \\
\hline 1 & 1 & 4 & 15 & 148,51 & 48,55 & 152,58 \\
2 & 2 & 3 & 15 & 35,44 & 10,54 & 36,66 \\
3 & 3 & 2 & 15 & 16,02 & 5,41 & 16,14 \\
\hline
\end{tabular}

Aplicando-se o algoritmo de Gibbs-com-Metropolis-Hastings (G$\mathrm{MH}$ ) foram geradas $m=10$ cadeias cada uma com tamanho $2 p=2000$. Para cada parâmetro consideraram-se as iterações $1010^{\text {th }}, 1020^{\text {th }}, \ldots, 2000^{\text {th }}$ desprezando-se as $p=1000$ primeiras iterações com a finalidade de diminuir o efeito dos pontos iniciais obtendo-se uma amostra G-MH de tamanho 1000. Na Tabela 9.19, apresenta-se um sumário da densidade a posteriori marginal aproximada obtida para os parâmetros $c_{1}, c_{2}, c_{3}$ e $\mathcal{P}$ e o monitoramento da convergência.

Como pode ser observado na Tabela 9.19 o valor de $\hat{R}$ está próximo de 1 para todos os parâmetros considerados no modelo o que indica segundo GELMAN \& RUBIN (1992) que a convergência foi atingida e, portanto, a amostra obtida via G-MH fornece sumários apropriados para a distribuições a posteriori marginal de interesse.

Na Tabela 9.20 apresentam-se os intervalos de credibilidade a $95 \%$ para $c_{1}, c_{2}, c_{3}$ e $\mathcal{P}$ obtidos quando se considera a amostra gerada segundo o algoritmo de Gibbs com Metropolis-Hastings utilizando-se a parametrização $\left(c_{1}, c_{2}, c_{3}, \mathcal{P}\right)$ e a reparametrização $\left(\theta_{1}=\ln c_{1}, \theta_{2}=\ln c_{2}, \theta_{3}=\ln c_{3}, \mathcal{P}\right)$

Na Tabela 9.20 observa-se que a amplitude do intervalo de credibilidade é menor quando se considera a parametrização $\theta=\left(\theta_{1}, \theta_{2}, \theta_{3}\right)$. 
As estimativas de Monte Carlo (7.19) para o tempo médio de vida sob o nível de estresse normal $V_{1}=1$, utilizando-se a amostra G-MH de tamanho 1000 na parametrização $\left(c_{1}, c_{2}, c_{3}\right)$, são dadas por $\hat{\mu_{1}}=9,76075$ e $\hat{\mu_{2}}=3,22346 \mathrm{e}$ na reparametrização $\left(\theta_{1}=\ln c_{1}, \theta_{2}=\ln c_{2}, \theta_{3}=\ln c_{3}\right)$ são dadas por $\hat{\mu_{1}}=9,308447$ e $\hat{\mu_{2}}=3,338447$.

Na Figura 9.17, apresenta-se a distribuição a posteriori marginal aproximada para $c_{1}, c_{2}, c_{3}$ e $\mathcal{P}$ considerando-se uma amostra G-MH de tamanho $S=1000$.
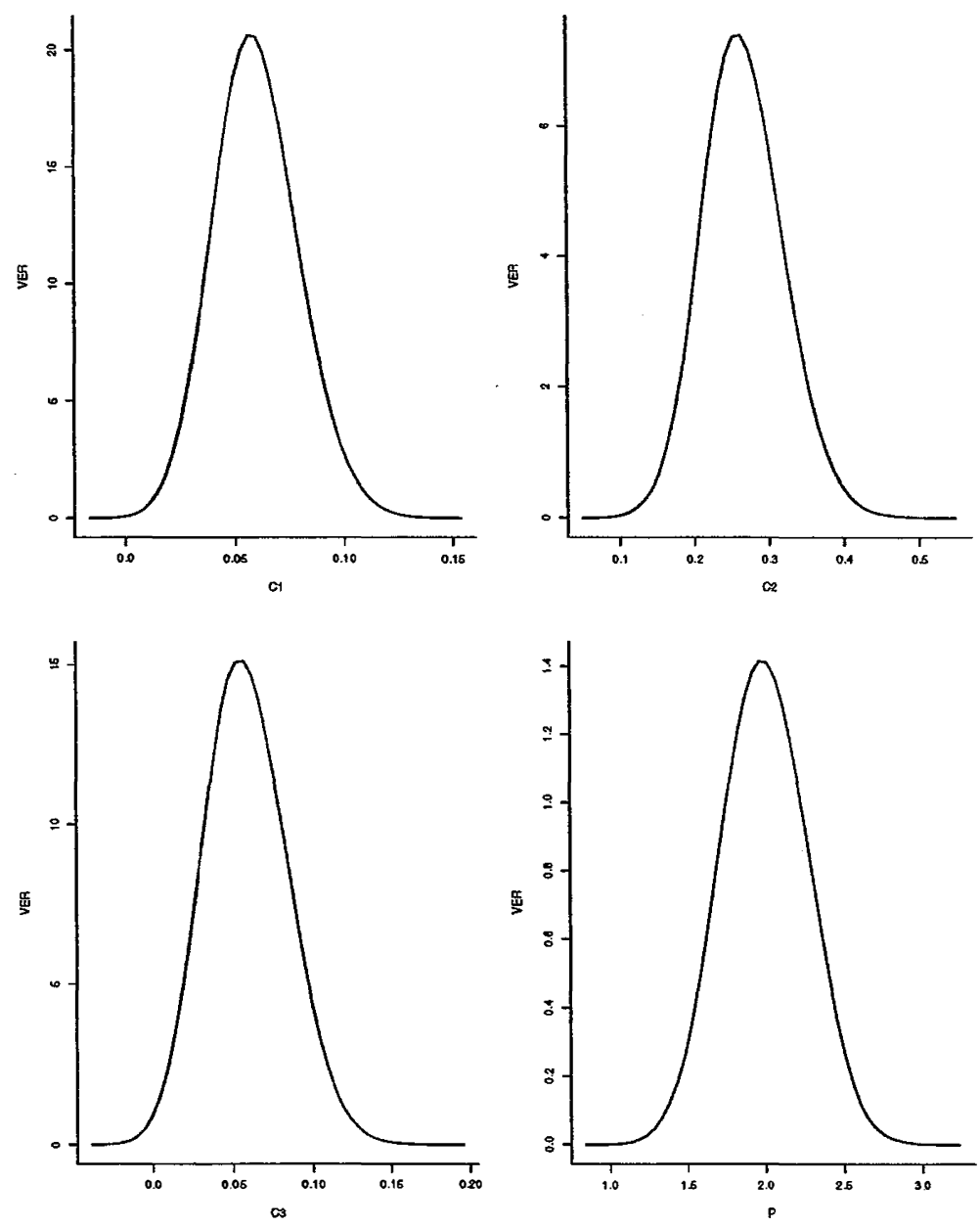

Figura 9.17: Gráfico das densidades a posteriori marginal aproximada para $c_{1}, c_{2}$, $c_{3}$ e $\mathcal{P}$ respectivamente 
Na Figura 9.18, apresenta-se a distribuição a posteriori marginal aproximada para $\theta_{1}, \theta_{2}, \theta_{3}$ e $\mathcal{P}$ considerando-se uma amostra G-MH de tamanho $S=1000$.
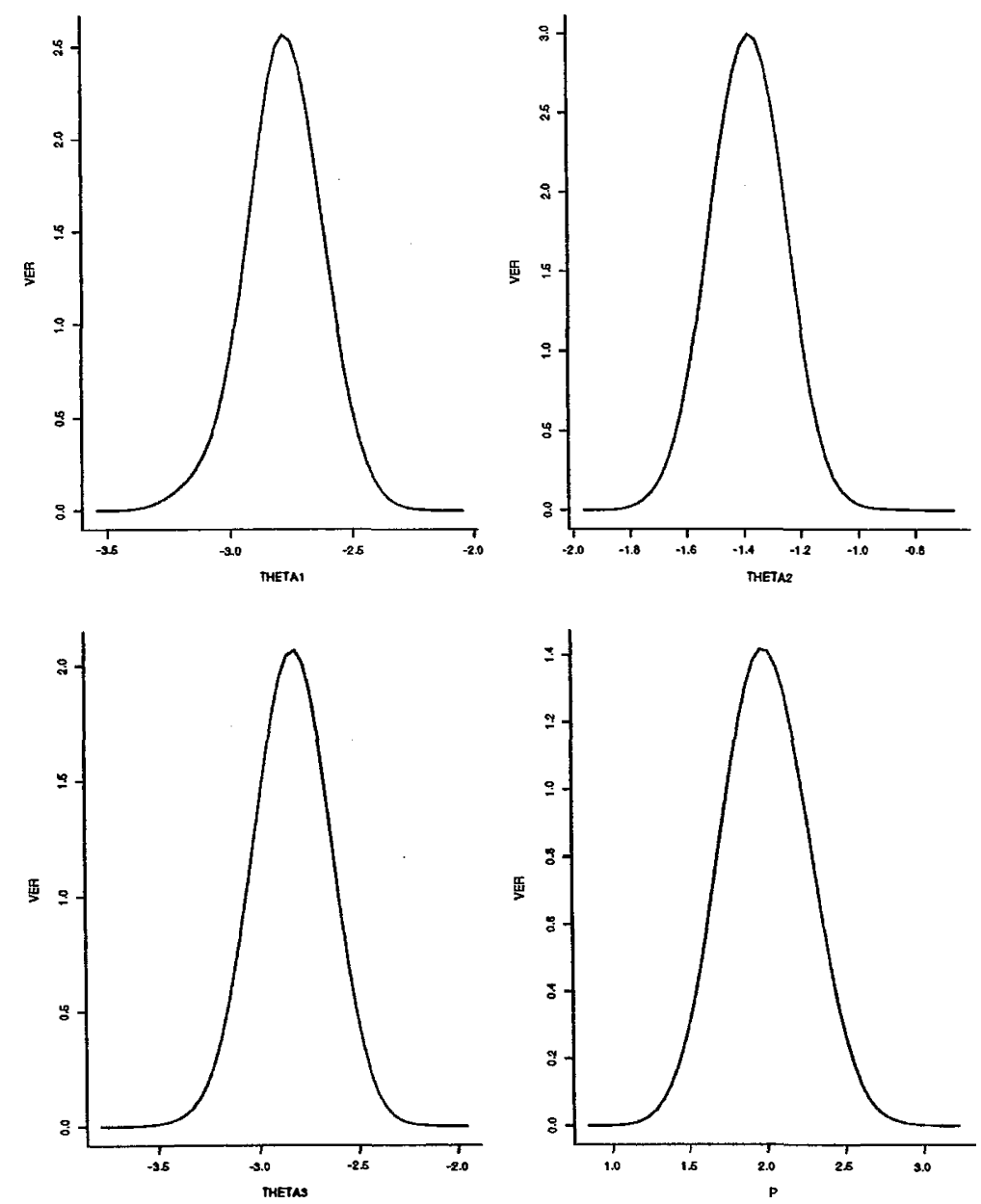

Figura 9.18: Gráficos das densidades a posteriori marginais aproximadas para $\theta_{1}$, $\theta_{2}, \theta_{3}, \mathcal{P}$ respectivamente

SANTANDER (1993) considera a análise clássica dos dados obtendo os estimadores de máxima verossimilhança através da verossimilhança maximizada para $\mathcal{P}$, isto é, utiliza o método de Newton-Raphson para calcular os estimadores de máxima verossimilhança $\hat{c_{1}}(\mathcal{P}), \hat{c_{2}}(\mathcal{P}), \hat{c_{3}}(\mathcal{P})$ para diferentes valores de $\mathcal{P}$ obtendo 
Tabela 9.19: Estatísticas da distribuição a posteriori marginal aproximada e monitoramento da convergência

\begin{tabular}{lcccc}
\hline Parâmetro & $c_{1}$ & $c_{2}$ & $c_{3}$ & $P$ \\
Mínimo & 0,0158 & 0,1409 & 0,0065 & 1,2820 \\
1o. Quartil & 0,0487 & 0,2353 & 0,0432 & 1,8610 \\
Mediana & 0,0589 & 0,2640 & 0,0571 & 1,9990 \\
Média & 0,0599 & 0,2669 & 0,0588 & 2,0080 \\
3o. Quartil & 0,0702 & 0,2954 & 0,0732 & 2,1530 \\
Máximo & 0,1213 & 0,4593 & 0,1507 & 2,7690 \\
Desvio-Padrão & 0,0519 & 0,1447 & 0,0683 & 0,6967 \\
\hline \multicolumn{5}{c}{ Monitoramento da Convergência } \\
\hline$\hat{R}$ & 1,0139 & 1,0151 & 1,0078 & 0,9989 \\
\hline
\end{tabular}

Tábela 9.20: Intervalos de confiança a $95 \%$ obtido via G-MH

\begin{tabular}{ccc}
\hline \multicolumn{3}{c}{ Parametrização } \\
\hline & $\left(c_{1}, c_{2}, c_{3}\right)$ & $\left(\theta_{1}, \theta_{2}, \theta_{3}\right)$ \\
\hline$c_{1}$ & $(0,03116 ; 0,09542)$ & $(0,04492 ; 0,08001)$ \\
$c_{2}$ & $(0,18867 ; 0,36606)$ & $(0,20074 ; 0,30751)$ \\
$c_{3}$ & $(0,02288 ; 0,10454)$ & $(0,04301 ; 0,07839)$ \\
$\mathcal{P}$ & $(1,60068 ; 2,46958)$ & $(1,56080 ; 2,42738)$ \\
\hline
\end{tabular}


os resultados apresentados na Tabela 9.21. Considera, também, a análise Bayesiana dos dados considerando a densidade a priori não informativa de Jeffreys (6.1). Assumindo $\mathcal{P}$ conhecido considera as seguintes densidades a priori informativas para os parâmetros: $c_{1} \sim \Gamma(5,72), c_{2} \sim \Gamma(15,54)$ e $c_{2} \sim \Gamma(3,41)$ obtendo-se os intervalos de credibilidade a $95 \%$ apresentados na Tabela 9.22 .

Comparando-se os intervalos de confiança e de credibilidade apresentados nas Tabelas 9.20, 9.21, 9.22 verifica-se que os resultados obtidos via amostra G-MH na parametrização $\left(c_{1}, c_{2}, c_{3}, \mathcal{P}\right)$ estão bastantes próximos dos intervalos de credibilidade a $95 \%$ quando se considera uma densidade a priori informativa para os parâmetros $c_{2}$ e $c_{3}$, o mesmo não ocorrendo para o parâmetro $c_{1}$. A análise Bayesiana apresentada por SANTANDER (1993) mostra que existem grandes diferenças nas inferências quando se considera informação a priori para os parâmetros $c_{1}, c_{2}, c_{3}$ considerando o parâmetro $\mathcal{P}$ fixo. Neste exemplo, nota-se que considerando-se informação a priori para todos os parâmetros do modelo: $c_{1}, c_{2}, c_{3}$ e $\mathcal{P}$ a amostra gerada através do algoritmo Gibbs-com-Metropolis-Hastings não causa grandes mudanças nas inferências.

Na Tabela 9.23, apresentam-se as estimativas de Monte Carlo (7.21) para o estimador de Bayes com relação à função de perda quadrática, para a função de confiabilidade para um sistema com dois componentes considerando-se um sistema em série $R_{S}\left(t_{0}\right)$ e em paralelo $R_{P}\left(t_{0}\right)$ sob o nível de estresse normal $V_{1}=1$ no tempo $t_{0}$ e as estimativas obtidas por SANTANDER (1993) .

Deve-se ressaltar que para obtenção da amostra G-MH e dos sumários a posteriori de interesse não foi necessária a utilização de métodos de aproximação nem de métodos numéricos mas "simplesmente" simulação iterativa, o que simplifica bastante a análise do modelo. 
Tabela 9.21: EMV e intervalo de confiança a $95 \%$

\begin{tabular}{ccc}
\hline & EMV & Intervalo de Confiança a $95 \%$ \\
\hline$c_{1}$ & 0,057127 & $(0,004260 ; 0,109994)$ \\
$c_{2}$ & 0,264144 & $(0,131705 ; 0,396584)$ \\
$c_{3}$ & 0,060240 & $(-0,009550 ; 0,130030)$ \\
$\mathcal{P}$ & 2,030000 & $(1,574531 ; 2,485469)$ \\
\hline
\end{tabular}

Tabela 9.22: Intervalos de confiança e de credibilidade para os parâmetros do modelo ACBVE em testes de vida acelerados com o modelo de potência

\begin{tabular}{c|cc}
\hline & \multicolumn{2}{|c}{ Intervalos de credibilidade $95 \%$} \\
\hline & Priori de Jeffreys & Priori Informativa \\
\hline$c_{1}$ & $(0,024 ; 0,122)$ & $(0,240 ; 0,300)$ \\
$c_{2}$ & $(0,161 ; 0,383)$ & $(0,195 ; 0,357)$ \\
$c_{3}$ & $(-0,017 ; 0,119)$ & $(0,022 ; 0,106)$ \\
$\mathcal{P}$ & $(1,592 ; 2,500)$ & $\ldots \ldots \ldots \ldots .$. \\
\hline
\end{tabular}

Tabela 9.23: Estimativas de Monte Carlos para a confiabilidade

\begin{tabular}{rllll}
\hline & & \multicolumn{2}{c}{ G-MH } \\
\hline tempo & $R_{S}\left(t_{0}\right)$ & $R_{P}\left(t_{0}\right)$ & $R_{S}\left(t_{0}\right)$ & $R_{P}\left(t_{0}\right)$ \\
\hline 1 & 0,6828 & 0,9756 & 0,6809 & 0,9750 \\
2 & 0,4602 & 0,9186 & 0,4648 & 0,9171 \\
3 & 0,3184 & 0,8460 & 0,3180 & 0,8439 \\
4 & 0,2174 & 0,7681 & 0,2182 & 0,7658 \\
5 & 0,1484 & 0,6908 & 0,1500 & 0,6827 \\
10 & 0,0220 & 0,3832 & 0,0239 & 0,3849 \\
\hline
\end{tabular}




\subsection{Exemplo 04}

Neste exemplo desenvolve-se a análise Bayesiana do modelo ACBVE (2.1) em testes de vida acelerados considerando o modelo de regressão dado por (8.1) utilizando o algoritmo "Gibbs-com-Metropolis-Hastings" apresentada no Capítulo 8. Considerando-se os dados de tempo de vida bivariados gerados considerando-se $\beta_{1}=$ $0.01, \beta_{2}=0.05, c_{1}=0.002, c_{2}=0.006$ e $c_{3}=0.009$ e a matriz de estresse $\mathbf{V}$ apresentados na Tabela 9.24. Observe que cada linha da matriz $\mathbf{V}$ representa uma condição de estresse na qual foram submetidos $n_{j}$ produtos.

$$
\mathrm{V}=\left(\begin{array}{rr}
-10 & 10 \\
0 & 10 \\
30 & 10 \\
-10 & 30 \\
0 & 30 \\
30 & 30 \\
-10 & 50 \\
\cdot & 50 \\
30 & 50
\end{array}\right)
$$

Para a análise Bayesiana via G-MH consideram-se as densidades $a$ priori dadas por (8.7): N1 b(2, $\left.c_{1} / c_{12}\right), c_{i} \sim \Gamma\left(a_{i}, b_{i}\right), \beta_{k} \sim N\left(\mu_{0 k}, \sigma_{0 k}^{2}\right)$ onde $a_{i}$, $b_{i}, i=1,2,3$ são valores apropriados e as densidades $a$ posteriori condicionais cheias dadas por (8.9), usando-se os dados apresentados na Tabela 9.25.

Seguindo-se procedimentos analógos aos apresentados nos exemplos 2 e 3 do Capítulo 9, gera-se uma amostra G-MH para a distribuição (8.8). Na Tabela 9.26 apresenta-se um sumário da distribuição a posteriori 
Tabela 9.24: Tabela de dados de tempo de vida gerados

\begin{tabular}{|c|c|c|c|c|c|}
\hline \multirow{2}{*}{\multicolumn{2}{|c|}{$\begin{array}{cc}x & y \\
V_{11}= & -10 ; V_{21}=10\end{array}$}} & \multirow{2}{*}{$\begin{array}{r}x \\
V_{12}=\end{array}$} & \multirow{2}{*}{$\begin{array}{c}y \\
V_{22}=10\end{array}$} & \multirow{2}{*}{\multicolumn{2}{|c|}{$\begin{array}{cc}x & y \\
V_{13}= & 30, V_{23}=10\end{array}$}} \\
\hline & & & & & \\
\hline 57,7766 & 50,6024 & 102,9000 & 95,7546 & 44,7246 & 37,5118 \\
\hline 30,4980 & 23,1823 & 102,2590 & 95,1141 & 1,8376 & 11,3359 \\
\hline 15,4739 & 7,8367 & 31,0630 & 23,7533 & 13,2085 & 5,4753 \\
\hline 79,2714 & 72,1200 & 17,9920 & 26,5806 & 35,7469 & 28,4910 \\
\hline 26,0849 & 18,7102 & 12,0230 & 4,2296 & 56,6761 & 49,4997 \\
\hline 31,3256 & 39,7426 & 16,7710 & 9,1805 & 62,4791 & 55,3127 \\
\hline 12,7805 & 21,5171 & 9,2560 & 1,2895 & 21,3563 & 13,8923 \\
\hline 24,6601 & 17,2619 & 16,2570 & 24,8867 & 9,4380 & 1,4846 \\
\hline 60,5910 & 53,4218 & 47,7180 & 40,5175 & 10,5767 & 2,6998 \\
\hline 9,7454 & 1,8138 & 5,6490 & 14,7689 & 25,5447 & 34,0128 \\
\hline$x$ & $y$ & $x$ & $y$ & $x$ & $y$ \\
\hline \multicolumn{2}{|c|}{$V_{14}=-10, V_{24}=30$} & \multicolumn{2}{|c|}{$V_{15}=0, V_{25}=30$} & \multicolumn{2}{|c|}{$V_{16}=-10, V_{26}=30$} \\
\hline 3,8839 & 13,1613 & 25,5541 & 18,1710 & 14,2563 & 6,5701 \\
\hline 27,2439 & 19,8866 & 27,5553 & 20,2024 & 9,3858 & 1,4287 \\
\hline 0,6602 & 10,3194 & 48,0861 & 40,8864 & 11,0007 & 3,1498 \\
\hline 13,5954 & 22,3037 & 6,8127 & 15,8480 & 24,0162 & 16,6065 \\
\hline 3,7031 & 12,9936 & 8,2092 & 0,1604 & 11,0740 & 3,2276 \\
\hline 14,7652 & 7,1002 & 11,3058 & 3,4729 & 14,2036 & 6,5152 \\
\hline 34,6080 & 27,3328 & 12,7174 & 4,9604 & 14,7183 & 7,0514 \\
\hline 21,4295 & 29,9532 & 23,2775 & 15,8538 & 10,7445 & 2,8781 \\
\hline 9,2034 & 1,2329 & 21,8704 & 14,4178 & 30,5760 & 38,9983 \\
\hline 4,9006 & 14,0814 & 4,7548 & 13,9481 & 8,6363 & 0,6224 \\
\hline$x$ & $y$ & $x$ & $y$ & $x$ & $y$ \\
\hline \multicolumn{2}{|c|}{$V_{17}=-10, V_{27}=50$} & \multicolumn{2}{|c|}{$V_{18}=0, V_{28}=50$} & \multicolumn{2}{|c|}{$V_{19}=-10, V_{29}=50$} \\
\hline 9,2096 & 1,23963 & 2,0914 & 11,5584 & 12,2095 & 20,9673 \\
\hline 11,4386 & 3,61338 & 9,6654 & 1,7281 & 8,5057 & 0,4814 \\
\hline 8,6549 & 0,64245 & 11,1968 & 3,3576 & 21,4919 & 14,0309 \\
\hline 10,2216 & 2,32202 & 14,2027 & 6,5143 & 10,1247 & 2,2187 \\
\hline 9,2695 & 1,30394 & 13,1331 & 5,3963 & 12,6162 & 4,8541 \\
\hline 10,2223 & 2,32271 & 8,0696 & 0,0091 & 8,1856 & 0,1349 \\
\hline 14,1025 & 6,40972 & 9,9314 & 2,0125 & 9,4584 & 1,5065 \\
\hline 8,4783 & 0,45175 & 12,8646 & 5,1148 & 10,6680 & 2,7968 \\
\hline 10,9192 & 3,06351 & 11,7797 & 3,9736 & 8,7267 & 0,7199 \\
\hline 14,3369 & 6,65412 & 8,7661 & 0,7624 & 8,3439 & 0,3064 \\
\hline
\end{tabular}


Tabela 9.25: Tabela de valores auxiliares

\begin{tabular}{crrrr}
\hline condição de estresse & $n \bar{x}$ & $n \bar{y}$ & $r$ & $R_{j}$ \\
\hline 1 & 348,2075 & 306,2087 & 2 & 365,3611 \\
2 & 361,8876 & 336,0753 & 3 & 388,2261 \\
3 & 281,5983 & 239,7159 & 2 & 299,5648 \\
4 & 133,9983 & 158,3650 & 6 & 188,6330 \\
5 & 190,1434 & 147,9213 & 2 & 208,3719 \\
6 & 148,6117 & 87,0480 & 1 & 157,0340 \\
7 & 106,8534 & 28,0232 & 0 & 106,8534 \\
8 & 101,7008 & 40,4271 & 1 & 111,1678 \\
9 & 110,3306 & 48,0170 & 1 & 119,0884 \\
\hline
\end{tabular}

Tabela 9.26: Estatísticas da distribuição a posteriori marginal aproximada e monitoramento da convergência

\begin{tabular}{llllll}
\hline Parâmetro & $c_{1}$ & $c_{2}$ & $c_{3}$ & $\beta_{1}$ & $\beta_{2}$ \\
$2,5 \%$ & 0,00193 & 0,00590 & 0,00893 & $-0,03636$ & 0,00693 \\
$25,0 \%$ & 0,00199 & 0,00598 & 0,00900 & $-0,00666$ & 0,03512 \\
$50,0 \%$ & 0,00203 & 0,00602 & 0,00904 & 0,01161 & 0,04911 \\
Média & 0,00203 & 0,00602 & 0,00904 & 0,00986 & 0,05115 \\
$75,0 \%$ & 0,00207 & 0,00605 & 0,00907 & 0,02773 & 0,06611 \\
$97,5 \%$ & 0,00215 & 0,00612 & 0,00914 & 0,05754 & 0,09896 \\
Desvio-Padrão & 0,00005 & 0,00005 & 0,00005 & 0,02506 & 0,02380 \\
\hline \multicolumn{7}{c}{ Monitoramento da convergência } \\
\hline$\hat{R}$ & 1,00000 & 1,00000 & 1,00000 & 1,00642 & 1,00322 \\
\hline
\end{tabular}



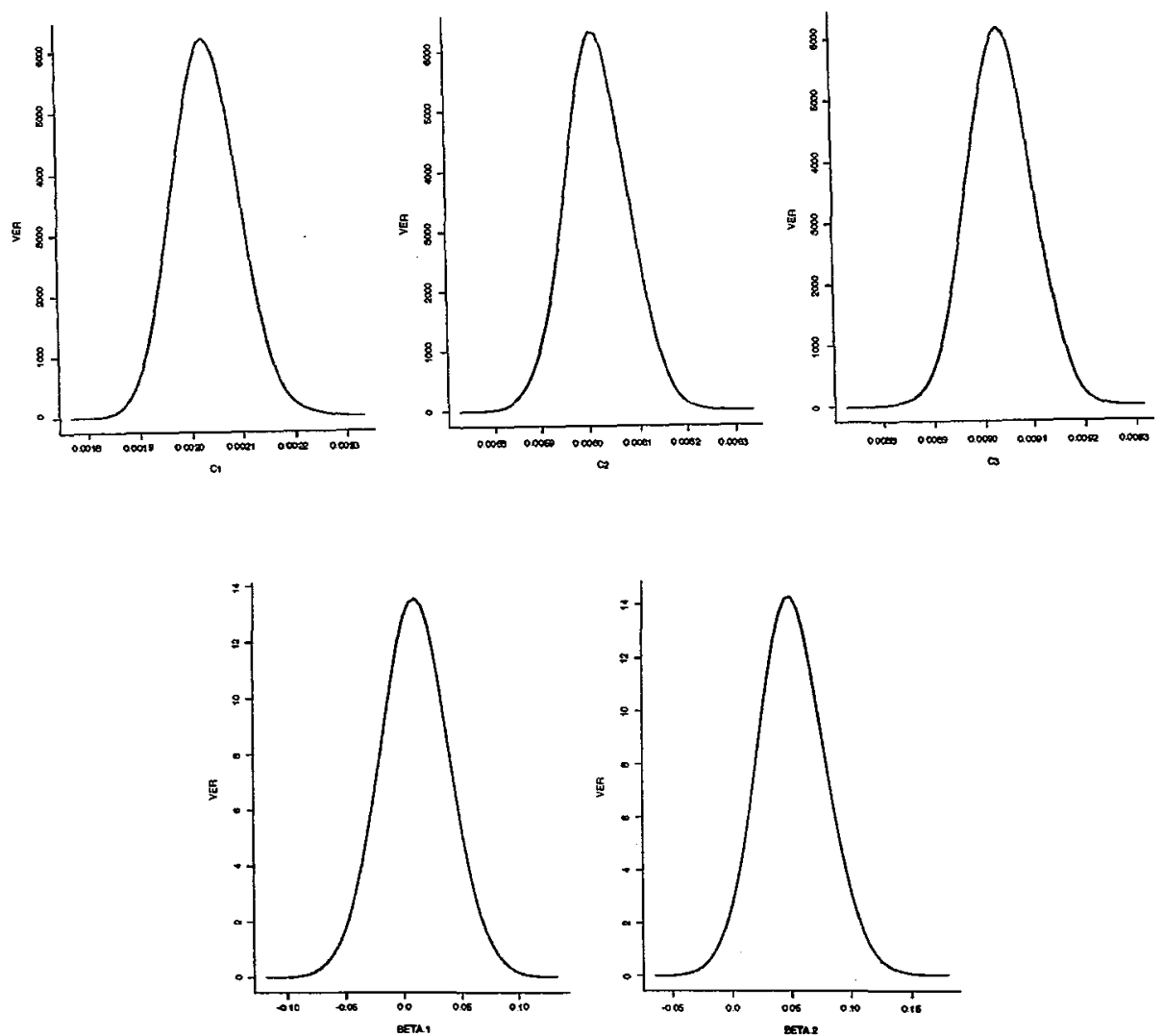

Figura 9.19: Gráficos das densidades a posteriori marginais aproximadas para $c_{1}$, $c_{2}, c_{3}, \beta_{1}, \beta_{2}$ respectivamente

marginal aproximada para os parâmetros $c_{1}, c_{2}, c_{3}, \beta_{1}, \beta_{2}$ obtida e o resultado do monitoramento da convergência. 


\section{Capítulo 10}

\section{Conclusões e Perspectivas}

\section{Futuras}

Analisadas cada uma das reparametrizações utilizadas neste trabalho chegou-se às conclusões que se seguem.

(i) Dentre a parametrização $\left(\lambda_{1}, \lambda_{2}, \lambda_{3}\right)$ e as reparametrizações $\left(\theta_{1}, \theta_{2}, \theta_{3}\right)$ e $\left(\phi_{1}, \phi_{2}, \phi_{3}\right)$, a melhor reparametrização para o modelo de ACBVE de BLOCK \& BASU foi obtida quando se considerou a reparametrização $\left(\phi_{1}, \phi_{2}, \phi_{3}\right)$ (Transformação de Box \& Cox) visto que apresentou melhor valor para STD e melhores resultados gráficos.

( ii) Dentre as parametrizações que envolvem a função de confiabilidade $\left(\lambda_{1}, \lambda_{2}, \theta_{0}\right)$ e $\left(\mu_{1}, \mu_{2}, \theta_{0}\right)$ ou reparametrizações desta tais como: $\left(\lambda_{1}, \lambda_{2}, \phi_{G J}\right)$ e $\left(\mu_{1}, \mu_{2}, \phi_{G J}\right)$ onde $\phi_{G J}$ é a transformação de Guerrero \& Johnson para $\theta_{0}$, aquelas que apresentaram melhores valores para STD foram aquelas onde foi considerada a transformação de GuerRero \& JoHnson para $\theta_{0}$.

(iii) Quando se considerou a reparametrização $\left(\mu_{1}, \mu_{2}, \phi_{G J}\right)$ onde $\phi_{G J}$ é a trans- 
formação de GUERRERo \& Johnson para $\theta_{0}$ muitos problemas computacionais foram encontrados para calcular os valores de STD visto que para seu cálculo é necessário calcular derivadas parciais de terceira ordem e estas apresentam expressões "bastante extensas" e complicadas, principalmente aquelas que envolvem o parâmetro $\phi_{G J}$. O tempo computacional médio gasto foi de aproximadamente 1000 segundos e a memória computacional de aproximadamente 30 megabytes para cada $t_{0}$ e $\xi_{i}$ fixos utilizando-se um programa computacional implementado no "software" MAPLEV versão 3 instalados em um microcomputador Pentium com 32 megabytes e $133 \mathrm{mHz}$. Devido às dificuldades apresentadas e ao valor de STD apresentados neste trabalho sugere-se que a informação do especialista, somente, seja utilizada para chutes iniciais para os parâmetros $\left(\lambda_{1}, \lambda_{2}, \theta_{0}\right)$.

( iv) Quando os valores para STD estão "razoavelmente" distantes como, por exemplo, os obtidos quando se considerou a parametrização $\left(\lambda_{1}, \lambda_{2}, \lambda_{3}\right)$ e reparametrização $\left(\theta_{1}, \theta_{2}, \theta_{3}\right)$ o número de iterações necessários para a convergência do método iterativo de Newton-Raphson aumentou quando se utilizou a reparametrização de maior valor para STD.

(v) Através dos Capítulos 6, 7, 8 pode-se verificar o quanto a simulação iterativa facilita a análise de modelos complexos. Mostrando que o algoritmo de Gibbs associado ao algoritmo de Metropolis-Hastings é bastante útil na análise Bayesiana porque facilita a obtenção de sumários a posteriori marginais de interesse. Deve-se ressaltar ainda que a implementação de programas computacionais não apresenta dificuldades adicionais nem com relação a memória ou velocidade dos microcomputadores utilizados.

Em um estudo futuro podem ser considerado outros modelos, como 
por exemplo, o modelo de Sarkar e através de técnicas Bayesianas e do algoritmo Gibbs-com-Metropolis-Hastings verificar a adequação e seleção de modelos. 


\section{Bibliografia}

ACHCAR, J.A. Inferences for accelerated life tests considering a bivariate exponential distribution, Statistics, 26, 269-283, fevereiro 1995.

ACHCAR, J.A.; LEAndro, R.A. Use of Markov Chain Monte Carlo Methods in a Bayesian Analysis of the BLOCK \& BASU Bivariate Exponential distribution. Notas do ICMSC. Série Estatística, 32, agosto 1996.

ACHCAR, J.A; SANTANDER, L.A.M. Use of approximate Bayesian methods for the Block and Basu bivariate exponential distribution, Journal of the Italian Statistical Society, 3, 233-250, dezembro 1993.

ACHCAR, J.A.; SMITH, A.F.M. Aspects of Reparametrization in Approximate Bayesian Inference. In: Geisser, S., Hodges, J.S, Press, S.J., Zellner, A. (Ed.) Essays in honor of G.A. Barnard. Amsterdam: North-Holland, 1990. p.431452.

Basu, A.P.; Ebrahimi, N. On a bivariate accelerated life test, Journal of Statistical Planning and Inference, 16, 297-304, 1987.

BICKel, P.J.; DoKsum, K.A. Mathematical Statistics - Basic Ideas and Selected Topics. California: Holden Day, 1977. 492p.

BLOCK, H.; BASU, A.P. A continuous bivariate exponential extension, Journal of the American Statistical Association, 69, 1031-1037, 348, dezembro 1974.

BLOCK, H.W. A characterization of a bivariate exponential distribution. The Annals of statistics, 5, 4, 808-12, 1977. 
Box, G.E.P; Cox, D.R. An Analysis of transformations. Journal of the Royal Statistical Society, B26, 211-252, 1964.

Box, G.E.P.; TIAO, G.C. Bayesian Inference in Satistical Analysis. Massachusetts: Addison-Wesley, 1973. 588p.

Chiang, C. L. Competing risks and conditional probabilities. Biometrics, 26, 767-776, 1970.

ChIB, S.; Greenberg, E. Understanding the Metropolis-Hastings algorithm, American Statistician, 49, 4, 327-335, novembro 1995.

Cox, D.R. Regression Models and Life Tables (with discussion). Journal of the Royal Statistical Society, B34, 187-220, 1972.

DAVID, H.A. On Chiang's proportionality assumption in the theory of competing risks. Biometrics, 26, 336-337, dezembro 1970.

Downton, F. Bivariate exponential distributions in reliability theory, Journal of the Royal Statistical Society, series B, 34, 408-417, 1970.

Epstein, B. Truncated life tests in the exponential case. Annals of Mathematical Statistical, 25, 555-564, junho 1954.

EpsteIn, B. Statistical life test acceptance procedures. Technometrics, 2, 435446, novembro, 1960.

Epstein, B.; Sobel, M. Life testing. Journal of the American Statistical Association, 48, 486-502, setembro 1953. 
Epstein, B.; SobeL, M. Some theorems relevant to life testing from an exponential distribution. Annals of Mathematical Statistical, 25, 373-381, junho 1954.

Epstein, B.; Sobel, M. Sequential life tests in the exponential case. Annals of Mathematical Statistical, 25, 373-381, março 1955.

FrEund, J.E. A bivariate extension of the exponential distribution, Journal of the American Statistical Association, 56, 971-977, dezembro 1961.

Gelfand, A.E.; SMith, A.F.M. Sampling-based approaches to calculating marginal densities. Journal of the American statistical Association, 85, 410, 398-409, junho 1990.

Gelman, A.E.; Rubin, D. Inference from iterative simulation using multiple sequences, Statistical Science, 7, 457-472, 1992.

Geman, S.; Geman, D. Stochastic relaxation, Gibbs distributions and the Bayesian restoration of images. IEEE Transactions on Pattern Analysis and Machine Intelligence, 6, 169-193, 1984.

Guerrero, V.M.; Johnson, R.A. Use of the Box-Cox Transformation with binary Response Models. Biometrika, 69, 309-314, 1982.

Gumbel, E.J. Bivariate exponential distributions, Journal of the American Statistical Association, 55, 698-707, dezembro 1960.

HAWKES, A.G. A bivariate exponential distribution with applications to reliability, Journal of the Royal Statistical Society, series B, 34, 129-131, 1972.

HILls, S.E.; SMITH, A.F.M. Diagnostic plots for improved parametrization in 
Bayesian inference. Biometrika, 80,1, 61-74, 1993.

KASS, R.E.; Slate, E.H. Reparametrization and diagnostic of posterior nonnormality. In: Bernardo, J.M.; Berger. J.; Dawid, A.P.; Smith, A.F.M. Bayesian Statistics 4. Oxford: Oxford University Press, 1992. p.627-633.

Kass, R.E.; Slate, E.H. Some diagnostics of maximum likelihood and posterior nonnormality. The Annals of Statistics, 22, 2, 668-695, 1994.

LaWless, J.F. Statistical Models and Methods for Lifetime Data. New York: John Wiley, 1982. 580p.

Leandro, R.A.; ACHCAR, J.A. Generation of bivariate lifetime data assuming the BLOCK \& BASU exponential distribution. Notas do ICMSC. Série Estatística, 19, julho 1995.

LEE, E.T. Statistical Methods for Survival Data Analysis. Second Edition. New York: John Wiley, 1992. 482p.

ManN, N.R.; Schafer, R.E; SingPuRWalla, N.D. Methods for Statistical Analysis of Reliability and Life Data. New York: John Wiley, 1974. 564p.

Marshall, A.W; OlKIN, I. A multivariate exponential distribution, Journal of the American Statistical Association, 62, 317, 30-44, março 1967.

Miller, R.G. Survival Analysis. New York: John Wiley, 1981.

Morgenstern D. Einfache Beispiele zweidimensionaler Verteilungen. Mitteilungsblatt für mathematische Statistik, 8, 234-35, 1956.

NAYLOR, J. C. \& SMITH, A.F.M. Applications of a method for the efficient computation of posterior distributions. Applied Statistics, 3, 2141-225, março 
1982.

Sarkar, S.K. A continuous bivariate exponential distribution, Journal of the American Statistical Association, 82, 667-675, 1987.

Santander, L.A.M. Testes de Sobrevivência Acelerados Bivariados. São Carlos, 1993. 160p. Dissertação (Mestrado). Instituto de Ciências Matemáticas de São Carlos, Universidade de São Paulo.

Smith, A.F.M.; RoberTs, G.O. Bayesian Computation via the Gibbs Sampler and related Markov Chain Monte Carlo Methods. Journal of the Royal Statistical Society, B55, 1, 3-23, outubro 1993.

Sukнatme, P.V. Tests of significance for samples of the $\chi^{2}$ population with two degress of freedom. Annals Eugenics, 8, 52-56, outubro, 1937.

TANneR, M.A.; Wong, W.H. The calculation of posterior distribution by data augmentation. Journal of the American Statistical Association, 82, 398, Junho 1987.

Tierney, L.; Kadane, J.B. Accurate approximation for posterior moments and marginal, Journal of the American Statistical Association, 81, 393, 82-86, 1986. 
Apêndices 


\section{Técnicas de aproximação e critérios de convergên-}

cia

\section{Método de Laplace}

Supondo que existe interesse em resolver a integral em $\theta$ dada por:

$$
\int f(\theta) e^{-n h(\theta)} d \theta
$$

Se $-n h(\theta)$ é uma função bem comportada com máximo no ponto $\hat{\theta}$ e $f(\theta)$ é uma função monótona positiva, o método de Laplace aproxima a integral (10.1) (ver por exemplo, TiERNEY \& KADANE, 1986) na forma:

$$
\int f(\theta) e^{-n h(\theta)} d \theta \propto\left(\frac{2 \pi}{n}\right) \sigma f(\hat{\theta}) e^{-n h(\hat{\theta})}
$$

$\sigma=\left(h^{\prime \prime}(\hat{\theta})\right)^{-1 / 2}$.

No caso multiparamétrico $\operatorname{com} \theta \in \Re^{m}$, a aproximação de Laplace é dada por:

$$
\int f(\theta) e^{-n h(\theta)} d \theta \propto(2 \pi)^{m / 2}\left\{\operatorname{det}\left[n D^{2} h(\hat{\theta})\right]\right\}^{-1 / 2} f(\hat{\theta}) e^{-n h(\hat{\theta})}
$$

onde $\hat{\theta}$ maximiza a função $-n h(\theta)$ e $D^{2} h(\theta)$ é a matriz Hessiana associada à função $h(\theta)$ avaliada em $\hat{\theta}$.

\section{Amostrador de Gibbs}

$\mathrm{Na}$ abordagem Bayesiana temos que a distribuição a posteriori é proporcional ao produto da verossimilhança e da distribuição a priori assim para obter momentos a posteriori de interesse nem sempre é uma tarefa muito fácil, 
visto que se a distribuição a priori não for uma distribuição conjugada ou outra que permita o cálculo direto dos momentos tem-se que recorrer a métodos numéricos ou de aproximações para obter os momentos a posteriori ou as marginais a posteriori de interesse. Este fato ocorre com grande frequência pois, em geral, as distribuições prioris devem refletir o conhecimento que se tem sobre $\boldsymbol{\theta}$ e daí tem-se, geralmente, distribuições a posteriori intratáveis. Um método bastante utilizado é o método de aproximação de Laplace introduzido por TiERNEY \& KADANE (1986). Este método, no entanto, torna-se bastante complicado quando a dimensão de $\boldsymbol{\theta}$ for maior que 2 pois para utilização do mesmo necessita-se determinar o máximo de funções que dependem de $\boldsymbol{\theta}$. Atualmente tem-se utilizado o algoritmo do amostrador de Gibbs o qual permite o cálculo dos momentos a posteriori de interesse sem a necessidade do cálculo da distribuição a posteriori marginal.

O amostrador de Gibbs é uma técnica para gerar variáveis aleatórias de uma distribuição marginal indiretamente, isto é, este método permite gerar variáveis aleatórias de uma distribuição marginal sem ter que calcular a sua densidade. Através da simulação de uma amostra suficientemente grande podem-se calcular a média, a variância e outras características dessa distribuição.

\section{Ilustração do amostrador de Gibbs}

Sejam $k$ variáveis aleatórias $\theta=\left\{\theta_{1}, \theta_{2}, \cdots, \theta_{k}\right\}$. Para gerar uma amostra aleatória de sua distribuição conjunta $p\left(\theta_{1}, \theta_{2}, \cdots, \theta_{k} \mid \mathcal{D}\right)$ via técnica de "Gibbs Sampling" deve-se proceder da seguinte maneira:

1. Chutar valores iniciais $\left\{\theta_{1}^{(0)}, \theta_{2}^{(0)}, \cdots, \theta_{k}^{(0)}\right\}$

2. Gerar $\theta_{1}^{(1)}$ da densidade condicional $p\left(\theta_{1} \mid \theta_{2}^{(0)}, \theta_{3}^{(0)}, \cdots, \theta_{k}^{(0)}, \mathcal{D}\right)$

3. Gerar $\theta_{2}^{(1)}$ da densidade condicional $p\left(\theta_{2} \mid \theta_{1}^{(1)}, \theta_{3}^{(0)}, \cdots, \theta_{k}^{(0)}, \mathcal{D}\right)$ 
4. Gerar $\theta_{k}^{(1)}$ da densidade condicional $p\left(\theta_{k} \mid \theta_{1}^{(1)}, \theta_{2}^{(1)}, \cdots, \theta_{k-1}^{(1)}, \mathcal{D}\right)$

5. Voltar ao passo (1) substituindo os valores iniciais por $\left\{\theta_{1}^{(1)}, \theta_{2}^{(1)}, \cdots, \theta_{k}^{(1)}\right\}$

6. Executar os passos (1)-(5) $t$ vezes obtendo-se assim o primeiro elemento da sequência Gibbs.

\section{Técnica de Gelman \& Rubin}

Teoricamente, Geman \& Geman (1984) mostram que o ponto $k$-dimensional $\left\{\theta_{1}^{(t)}, \theta_{2}^{(t)}, \ldots, \theta_{k}^{(t)}\right\}$ produzido na $t$-ésima iteração do esquema amostral converge em distribuição para uma variável aleatória da distribuição $p\left(\theta_{1}, \theta_{2}, \cdots, \theta_{k}, \mathcal{D}\right)$ se $t$ for suficientemente grande. Além disso, $\theta_{i}^{t}$ pode ser considerado como uma observação simulada de $p\left(\theta_{i} \mid \mathcal{D}\right)$, a distribuição marginal de $\theta_{i}$. Replicando o processo $2 n$ vezes obtemos $2 n$ vetores,

$$
\left\{\theta_{1}^{(t)}, \theta_{2}^{(t)}, \cdots, \theta_{k}^{(t)}\right\}
$$

Da convergência do amostrador de Gibbs qualquer característica da densidade marginal $p\left(\theta_{i} \mid \mathcal{D}\right)$ pode ser obtida. Na prática, entretanto, ainda existe muita discussão sobre o monitoramento da convergência.

Neste trabalho foi utilizada a técnica proposta por Gelman \& RUBiN (1992). Supondo-se que foram geradas $m(m \geq 2)$ sequências Gibbs cada uma delas com diferentes valores iniciais amostrados de uma distribuição dispersa com tamanho $2 n$. A técnica de Gelman \& RUBin descarta os $n$ primeiros valores de cada sequência com a finalidade de diminuir o efeito dos valores iniciais ou ainda para o processo entrar em estabilidade e trabalha com os $n$ valores restantes. Para cada parâmetro de interesse, calcula: 
1. $B / n=$ a variância entre as $m$ médias das $m$ sequências, $\hat{\theta_{i .}}$, cada uma delas baseada em $n$ valores de $\theta$,

$$
\frac{B}{n}=\frac{\sum_{i=1}^{m}\left(\bar{\theta}_{i .}-\bar{\theta}_{. .}\right)^{2}}{m-1}
$$

2. $W=$ a média das $m$ variâncias dentro de cada sequência, $s_{i}^{2}$, baseadas em $n-1$ graus de liberdade,

$$
W=\frac{\sum_{1}^{n} s_{i}^{2}}{m} \text { onde } s_{i}^{2}=\frac{\sum_{i=1}^{m}\left(\theta_{i}-\overline{\theta_{i .}}\right)^{2}}{n-1}
$$

note que se $m=1$ será impossível calcular $B$.

3. Assim, a variância da distribuição estudada pode ser estimada por

$$
\hat{\sigma}^{2}=\frac{n-1}{n} W+\frac{1}{n} B
$$

e sua média é estimada por $\hat{\mu}=\overline{\theta_{\text {.. }}}$

$\mathrm{O}$ resultado é que $p(\boldsymbol{\theta} \mid \mathcal{D})$ tem uma distribuição aproximadamente $t$-student com centro em $\hat{\mu}$, com escala $\sqrt{\hat{V}}=\sqrt{\hat{\sigma}^{2}+B / m n}$ e graus de liberdade $d f=2 \hat{V}^{2} / \widehat{\operatorname{var}}(\hat{V})$ onde

$$
\begin{gathered}
\widehat{\operatorname{var}}(\hat{V})=\left(\frac{n-1}{n}\right)^{2} \frac{1}{m} \widehat{\operatorname{var}}\left(s_{i}^{2}\right)+\left(\frac{m+1}{m n}\right)^{2} \frac{2}{m-1} B^{2}+2 \frac{(m+1)(n-1)}{m n^{2}} \\
\frac{n}{m}\left[\widehat{\operatorname{cov}}\left(s_{i}^{2}, \bar{x}_{i .}^{2}\right)-2 \bar{x}_{. .} \widehat{\operatorname{cov}}\left(s_{i}^{2}, \bar{x}_{i .}\right)\right]
\end{gathered}
$$

e onde a variância estimada e a covariância são obtidas dos $m$ valores amostrais de $\bar{x}_{i .}$ e $s_{i}^{2} ; d f \longrightarrow \infty$ quando $n \longrightarrow \infty$.

Monitorando a convergência da simulação iterativa pela estimativa do fator pelo qual a escala da distribuição estudada $p(\theta \mid \mathcal{D})$ poderia ser reduzida se 
as simulações continuassem no limite $n \longrightarrow \infty$. Esta escala de redução é estimada por:

$$
\hat{R}=\sqrt{(\hat{V} / W) d f /(d f-2)}
$$

a qual tende a 1 quando $n \longrightarrow \infty$. $\hat{R}$ é o quociente entre a variância atual pela variância dentro da sequência, $\hat{V}$,

Se o fator $\sqrt{\hat{R}}$ é alto, devemos considerar mais simulações para melhorar a inferência sobre a distribuição de interesse. Se $\hat{R} \approx 1$ a convergência ocorre e as amostras selecionadas das iterações são i.i.d. .

A seguir apresenta-se um programa implementado no software SPlus para verificação da convergência. Observe-se que os programas: monitor, colmeans, colvars, cov, gpar são subroutinas do programa "S Functions for inference from iterative simulation" escrito por ANDREw Gelman, DePt. Of Statistics, UC, BERKELEY, podendo ser adquirido através do e-mail: gelman@stat.berkeley.edu, devendo estar gravados no drive A: e o arquivo: nomearq deve ser escrito em ASCII e estar com o seguinte formato:

$\begin{array}{cccc}\text { Parametro }_{1} & \text { Parametro }_{2} & \ldots & \text { Parametro }_{n} \\ \mathrm{~d} 11 & \mathrm{~d} 12 & \ldots & \mathrm{d} 1 \mathrm{~m} \\ \ldots & \ldots & \ldots & \ldots \\ \mathrm{dn} 1 & \mathrm{dn} 2 & \ldots & \mathrm{dnm}\end{array}$

$>$ monitor<-source("a: IMmonitor")

$>$ col.means<-source("k: Igelman I/colmeans")

$>$ col.vars<-source("a: \lcolvars")

$>$ cov<-source $(" \mathrm{a}:$ : Ilcov")

$>$ chisqdf<-source("a:Mchisqdf")

$>$ gpar<-source("a: IIgpar")

$>\mathrm{r}<-\operatorname{matrix}(\mathrm{scan}$ ("a: \Inomearq"), byrow= $\mathrm{T}, \mathrm{ncol}=\mathrm{m}$ ) 
$>\mathrm{r}<-\mathrm{r}[, 1: \mathrm{m}]$

$>$ a<-as.vector $(r)$

$>\operatorname{dim}(\mathrm{a})<-c(k n, k, m)$

$>$ monitor(a)

$>$ \#gpar(a) (Opcional)

\section{Algoritmo de Metropolis-Hastings}

Nos problemas apresentados nesse trabalho quando se consideram as distribuições condicionais necessárias para aplicação da técnica do algoritmo Gibbs verifica-se que estas não possuem uma distribuição padrão, no sentido de que seja fácil gerar valores de variáveis que tenham essa distribuição; assim foi necessário utilizar outra metodologia para obter os valores amostrais desejados. Para tanto foi utilizado neste trabalho o algoritmo de "Metropolis- Hastings". De acordo com Chib \& Greenberg (1995) este algoritmo é um método poderoso que tem por base as cadeias de Markov para simular uma distribuição de interesse. Com a finalidade de gerar valores amostrais da distribuição de interesse é necessário explorar a forma conhecida da distribuição para especificar uma densidade geradora candidata (ver por exemplo, ChiB \& Greenberg,1995). Por exemplo, se a distribuição condicional de interesse, $\phi(t)$ puder ser fatorada como $\pi(t)=\psi(t) h(t)$ onde $h(t)$ é uma distribuição que pode ser facilmente amostrada e se $\psi(t)$ for uniformemente limitada então poderemos amostrar $\pi(t)$ seguindo os seguintes passos:

1. Atribuir um valor inicial para $t, t^{0}$.

2. Gerar um novo valor para $t, t^{G}$.

3. Considerar $p=\min \left\{\frac{\psi\left(t^{G}\right)}{\psi\left(t^{0}\right)}, 1\right\}$ 
4. Gerar uma valor $b=$ Bernoulli $(p)$.

5. Considerar o novo valor para $t, t^{1}=b t^{G}+(1-b) t^{0}$

6. Voltar ao passo 2 até a convergência ser atingida. 


\section{Alguns programas utilizados}

Apresentam-se a seguir alguns programas computacionais utilizados nos exemplos apresentados no Capítulo 9. Os "softwares" utilizados foram SAS, Maple V e o Splus.

\section{Programa 1}

Este programa implementado no "software" SAS é utilizado para simular os dados de tempo de vida bivariados apresentados na Tabela 1. Para sua implementação segue-se, basicamente, os passos apresentados no Capítulo 4.

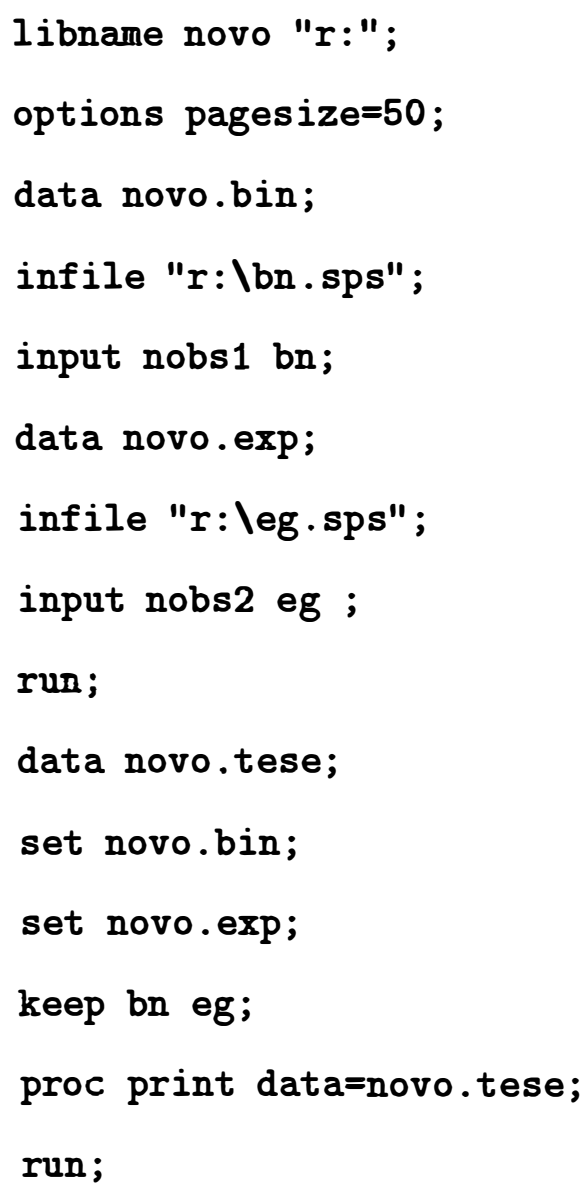




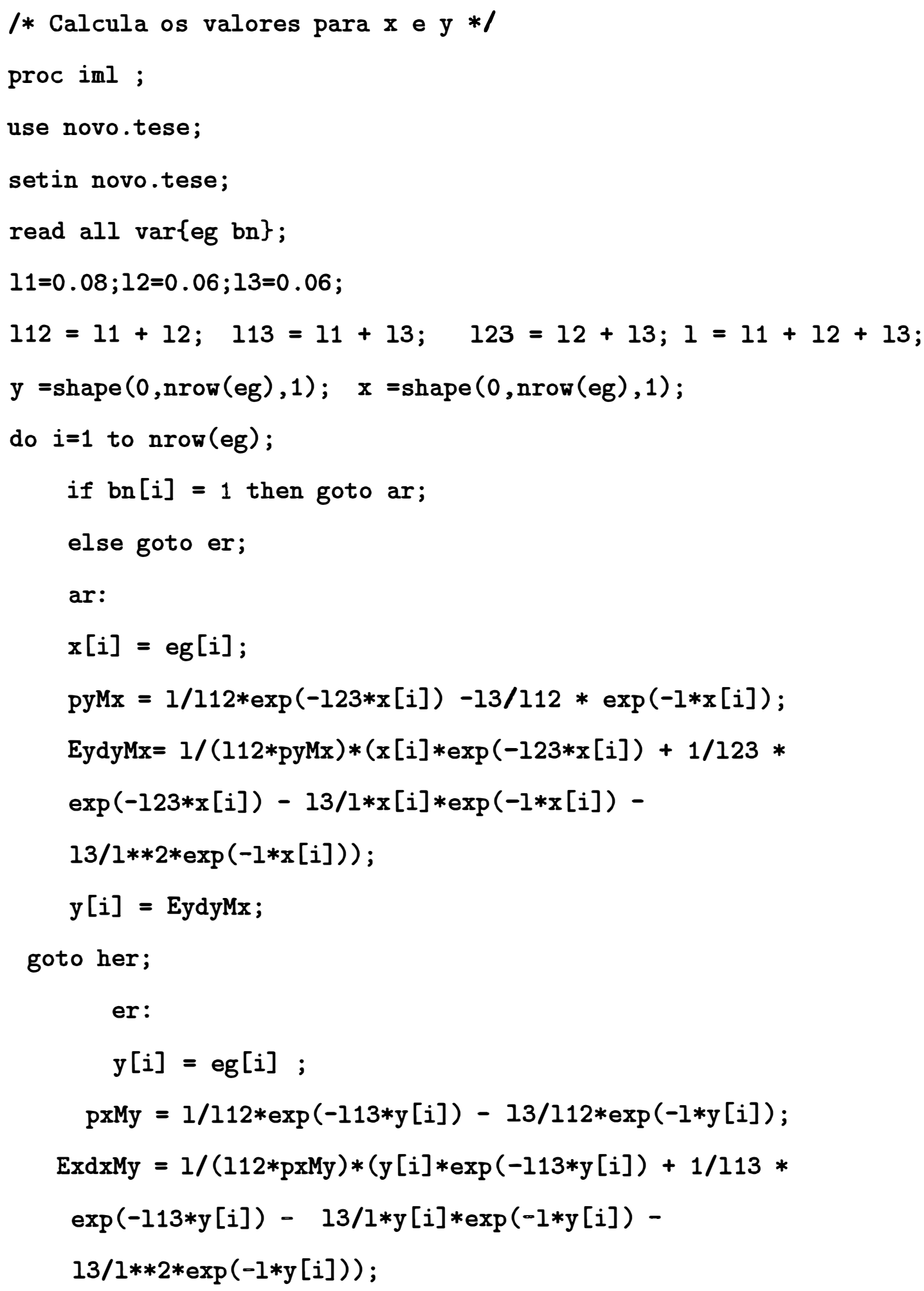




$$
x[i]=\operatorname{ExdxMy}
$$

her:

end;

$\operatorname{arq} 1=e g|| b n|| x|| y$;

$r=\left\{\begin{array}{llll}\text { eg } & \mathrm{x} & \mathrm{y}\end{array}\right\}$;

create novo.arq1 from arq1 [colname=r];

append from arq1;

quit;

proc sort ascii data=novo.arq1 out=novo.arq2;

by eg;

run;

proc iml;

use novo.arq2;

setin novo.arq2;

read all var\{eg bn $\mathrm{x} y\}$;

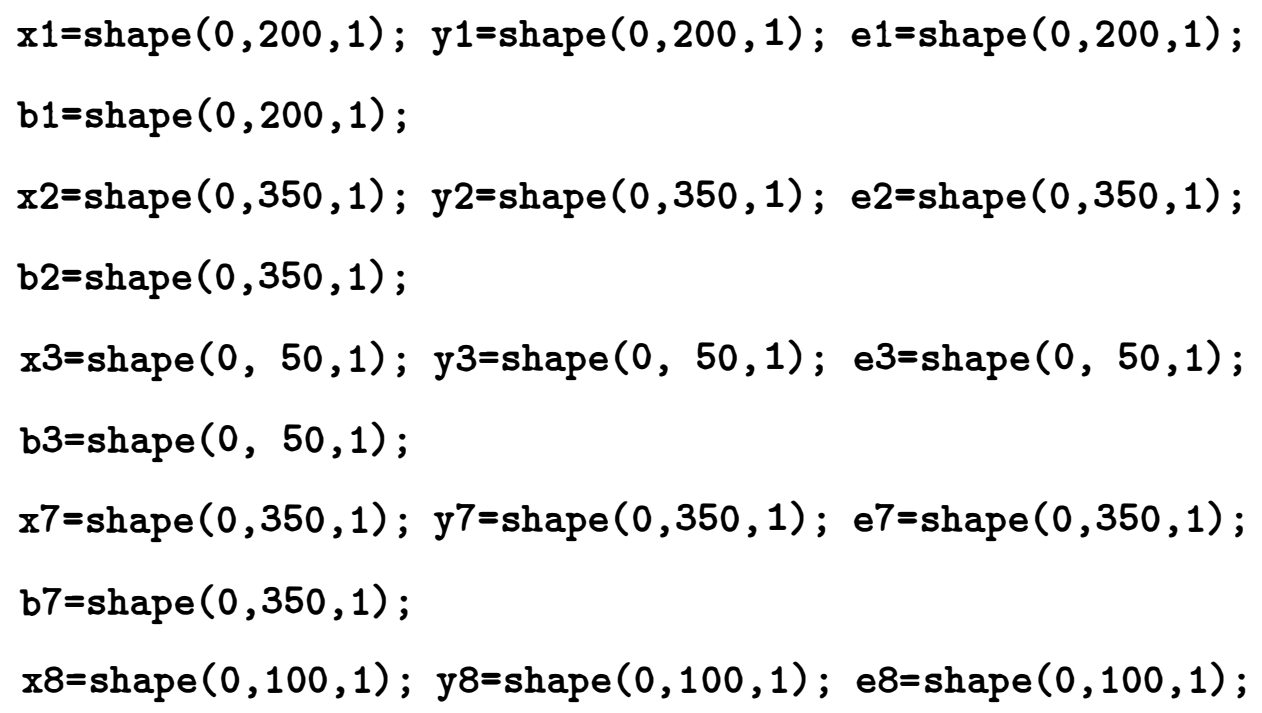




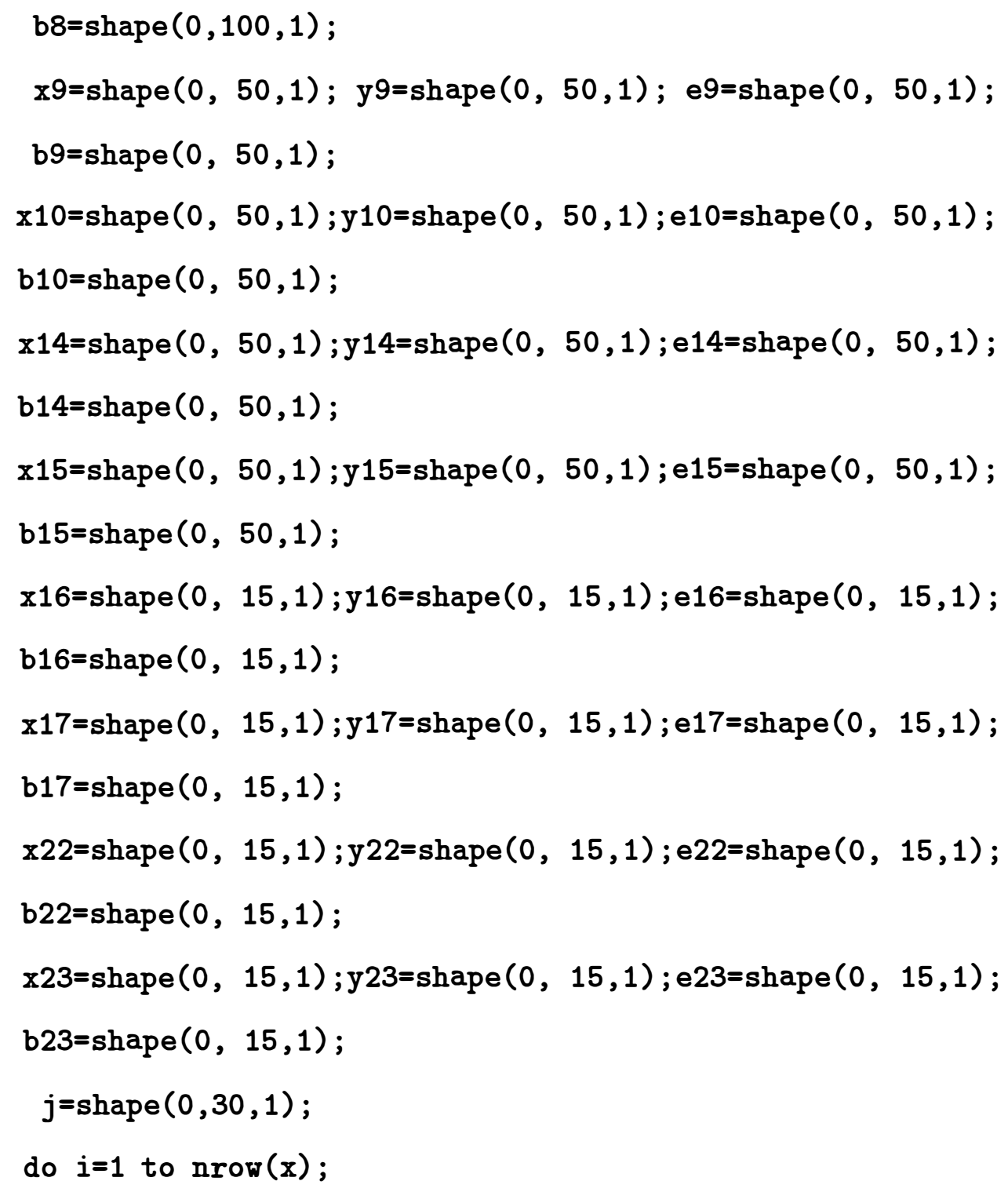


else if $\mathrm{x}[\mathrm{i}]>7.5 \& \mathrm{x}[\mathrm{i}]<=15.0 \& \mathrm{y}[\mathrm{i}]>11$ \& $\mathrm{y}[\mathrm{i}]<=22$ then goto de8; else if $x[i]>15.0 \& x[i]<=22.5 \& y[i]>11 \& y[i]<=22$ then goto de9; else if $\mathrm{x}[\mathrm{i}]>22.5 \& \mathrm{x}[\mathrm{i}]<=30.0$ \& $\mathrm{y}[\mathrm{i}]>11$ \& $\mathrm{y}[\mathrm{i}]<=22$ then goto de10; else if $\mathrm{x}[\mathrm{i}]>30.0 \& \mathrm{x}[\mathrm{i}]<=37.5 \& \mathrm{y}[\mathrm{i}]>11$ \& $\mathrm{y}[\mathrm{i}]<=22$ then goto de11; else if $x[i]>37.5 \& x[i]<=45.0$ \& $y[i]>11$ \& $y[i]<=22$ then goto de12; else if $x[i]>0.0 \& x[i]<=7.5 \& y[i]>22 \& y[i]<=33$ then goto de13; else if $x[i]>7.5 \& x[i]<=15.0 \& y[i]>22 \& y[i]<=33$ then goto de14; else if $x[i]>15.0 \& x[i]<=22.5 \& y[i]>22 \& y[i]<=33$ then goto de15; else if $x[i]>22.5 \& x[i]<=30.0$ \& $y[i]>22 \& y[i]<=33$ then goto de16; else if $x[i]>30.0 \& x[i]<=37.5 \& y[i]>22 \& y[i]<=33$ then goto de17; else if $x[i]>37.5 \& \mathrm{x}[i]<=45.0$ \& $y[i]>22$ \& $y[i]<=33$ then goto de18; else if $\mathrm{x}[\mathrm{i}]>0.0 \& \mathrm{x}[\mathrm{i}]<=7.5 \& \mathrm{y}[\mathrm{i}]>33$ \& $\mathrm{y}[\mathrm{i}]<=44$ then goto de19; else if $x[i]>7.5 \& \mathrm{x}[i]<=15.0$ \& $y[i]>33$ \& $y[i]<=44$ then goto de20; else if $\mathrm{x}[\mathrm{i}]>15.0$ \& $\mathrm{x}[\mathrm{i}]<=22.5$ \& $\mathrm{y}[\mathrm{i}]>33$ \& $\mathrm{y}[\mathrm{i}]<=44$ then goto de21; else if $x[i]>22.5 \& \mathrm{x}[i]<=30.0 \& \mathrm{y}[i]>33 \& \mathrm{y}[i]<=44$ then goto de22; else if $\mathrm{x}[\mathrm{i}]>30.0 \& \mathrm{x}[\mathrm{i}]<=37.5 \& \mathrm{y}[i]>33$ \& $\mathrm{y}[\mathrm{i}]<=44$ then goto de23; else if $x[i]>37.5 \& \mathrm{x}[i]<=45.0$ \& $y[i]>33$ \& $y[i]<=44$ then goto de24; else if $\mathrm{x}[\mathrm{i}]>0.0 \& \mathrm{x}[\mathrm{i}]<=7.5 \& \mathrm{y}[\mathrm{i}]>44$ \& $\mathrm{y}[\mathrm{i}]<=55$ then goto de25; else if $\mathrm{x}[\mathrm{i}]>7.5$ \& $\mathrm{x}[\mathrm{i}]<=15.0$ \& $\mathrm{y}[i]>44$ \& $\mathrm{y}[\mathrm{i}]<=55$ then goto de26; else if $\mathrm{x}[\mathrm{i}]>15.0$ \& $\mathrm{x}[\mathrm{i}]<=22.5$ \& $\mathrm{y}[i]>44$ \& $\mathrm{y}[\mathrm{i}]<=55$ then goto de27; else if $x[i]>22.5 \& \mathrm{x}[i]<=30.0$ \& $y[i]>44$ \& $y[i]<=55$ then goto de28; else if $x[i]>30.0 \& x[i]<=37.5 \& y[i]>44 \& y[i]<=55$ then goto de29; else if $x[i]>37.5 \& \mathrm{x}[i]<=45.0$ \& $y[i]>44$ \& $y[i]<=55$ then goto de $30 ;$ else print "fora da tabulacao" i; $\operatorname{de1}: j[1]=j[1]+1 ; x 1[j[1]]=x[i] ; y 1[j[1]]=y[i]$; $e 1[j[1]]=e g[i] ; b 1[j[1]]=b n[i]$; goto fim; 
$\operatorname{de} 2: j[2]=j[2]+1 ; x 2[j[2]]=x[i] ; y 2[j[2]]=y[i]$;

$e 2[j[2]]=e g[i] ; b 2[j[2]]=b n[i]$; goto fim;

de3: $j[3]=j[3]+1 ; x 3[j[3]]=x[i] ; y 3[j[3]]=y[i]$;

$e 3[j[3]]=e g[i] ; b 3[j[3]]=b n[i]$; goto fim;

$\operatorname{de} 4: j[4]=j[4]+1$; goto $f$ im;

de5: $j[5]=j[5]+1$; goto $f$ im;

de6: $j[6]=j[6]+1$; goto fim;

de7: $j[7]=j[7]+1 ; x 7[j[7]]=x[i] ; y 7[j[7]]=y[i]$;

$e 7[j[7]]=e g[i] ; b 7[j[7]]=b n[i]$; goto fim;

$\operatorname{de} 8: j[8]=j[8]+1 ; x 8[j[8]]=x[i] ; y 8[j[8]]=y[i]$;

$e 8[j[8]]=e g[i] ; b 8[j[8]]=b n[i]$; goto fim;

$\operatorname{de9}: j[9]=j[9]+1 ; x 9[j[9]]=x[i] ; y 9[j[9]]=y[i]$;

$e 9[j[9]]=e g[i] ; b 9[j[9]]=b n[i]$; goto fim;

$\operatorname{de10}: j[10]=j[10]+1 ; x 10[j[10]]=x[i] ; y 10[j[10]]=y[i]$;

$e 10[j[10]]=e g[i] ; b 10[j[10]]=b n[i] ;$ goto $f i m ;$

$\operatorname{de11}: j[11]=j[11]+1$; goto $\mathrm{fim}$;

de12: $j[12]=j[12]+1$; goto fim;

de13: $j[13]=j[13]+1$; goto fim;

$\operatorname{de14}: j[14]=j[14]+1 ; x 14[j[14]]=x[i] ; y 14[j[14]]=y[i]$;

$e 14[j[14]]=e g[i] ; b 14[j[14]]=b n[i]$; goto $f i m$;

$\operatorname{de15}: j[15]=j[15]+1 ; x 15[j[15]]=x[i] ; y 15[j[15]]=y[i]$;

$e 15[j[15]]=e g[i] ; b 15[j[15]]=b n[i] ;$ goto fim;

$\operatorname{de16}: j[16]=j[16]+1 ; x 16[j[16]]=x[i] ; y 16[j[16]]=y[i]$;

$e 16[j[16]]=e g[i] ; b 16[j[16]]=b n[i] ;$ goto fim;

$\operatorname{de} 17: j[17]=j[17]+1 ; x 17[j[17]]=x[i] ; y 17[j[17]]=y[i]$;

$e 17[j[17]]=e g[i] ; b 17[j[17]]=b n[i]$; goto fim; 
de18: $j[18]=j[18]+1$; goto fim;

de19: $j[19]=j[19]+1$; goto fim;

de20: $j[20]=j[20]+1$; goto $f$ im ;

$\operatorname{de21}: j[21]=j[21]+1$; goto fim;

$\operatorname{de} 22: j[22]=j[22]+1 ; x 22[j[22]]=x[i] ; y 22[j[22]]=y[i] ;$

$e 22[j[22]]=e g[i] ; b 22[j[22]]=b n[i] ;$ goto fim;

$\operatorname{de23}: j[23]=j[23]+1 ; x 23[j[23]]=x[i] ; y 23[j[23]]=y[i]$;

$e 23[j[23]]=e g[i] ; b 23[j[23]]=b n[i] ;$ goto $f i m$;

$\operatorname{de} 24: j[24]=j[24]+1$; goto fim;

$\operatorname{de25:} j[25]=j[25]+1$; goto fim;

de26: $j[26]=j[26]+1$; goto $f i m$;

$\operatorname{de} 27: j[27]=j[27]+1$; goto $f i m$;

de28: $j[28]=j[28]+1$; goto fim;

$\operatorname{de29}: j[29]=j[29]+1$; goto fim;

$\operatorname{de} 30: j[30]=j[30]+1$; goto fim;

fim:

end ;

$n=\operatorname{sum}(j)$;

$a 1=j[1] / / j[2] / / j[3] / / j[4] / / j[5] / / j[6]$;

$a 2=j[7] / / j[8] / / j[9] / / j[10] / / j[11] / / j[12]$;

a3 $=j[13] / / j[14] / / j[15] / / j[16] / / j[17] / / j[18]$;

$a 4=j[19] / / j[20] / / j[21] / / j[22] / / j[23] / / j[24]$;

$a 5=j[25] / / j[26] / / j[27] / / j[28] / / j[29] / / j[30]$;

$a=a 1|| a 2|| a 3|| a 4|| a 5$;

print a $\mathrm{n}$;

$j 1=0 ; j 2=0 ; j 3=0 ; j 4=0 ; j 5=0 ; j 6=0 ; j 7=0 ; j 8=0 ; j 9=0 ;$ 
do $i=1$ to 1000 ;

if $x[i]>0.0 \& x[i]<=7.5 \& y[i]>0 \& y[i]<=11$ then goto fe1; else if $x[i]>7.5 \& x[i]<=15.0 \& y[i]>0 \& y[i]<=11$ then goto $f e 2$; else if $\mathrm{x}[\mathrm{i}]>15.0$ \& $\mathrm{x}[\mathrm{i}]<=45.0$ \& $\mathrm{y}[\mathrm{i}]>0$ \& $\mathrm{y}[\mathrm{i}]<=11$ then goto $\mathrm{fe} 3$; else if $\mathrm{x}[\mathrm{i}]>0.0 \& \mathrm{x}[\mathrm{i}]<=7.5 \& \mathrm{y}[\mathrm{i}]>11 \& \mathrm{y}[\mathrm{i}]<=22$ then goto fe4; else if $x[i]>7.5 \& \mathrm{x}[i]<=15.0 \& \mathrm{y}[i]>11 \& \mathrm{y}[i]<=22$ then goto $f e 5 ;$ else if $\mathrm{x}[i]>15.0 \& \mathrm{x}[\mathrm{i}]<=45.0 \& \mathrm{y}[i]>11 \& \mathrm{y}[i]<=22$ then goto fe6; else if $x[i]>0.0 \& x[i]<=7.5 \& y[i]>22 \& y[i]<=55$ then goto $f e 7$; else if $x[i]>7.5 \& x[i]<=15.0 \& y[i]>22 \& y[i]<=55$ then goto fe8; else if $\mathrm{x}[\mathrm{i}]>15.0 \& \mathrm{x}[i]<=60.0 \& \mathrm{y}[i]>22 \& \mathrm{y}[i]<=55$ then goto fe9; else print "fora da tabulacao" i;

fe1: $j 1=j 1+1$; goto $f i m$;

fe2: $j 2=j 2+1$; goto $f i m$;

fe3: $j 3=j 3+1$; goto $f i m$;

fe4: $j 4=j 4+1$; goto $f i m$;

fe5: $j 5=j 5+1$; goto $f i m$;

fe6: $j 6=j 6+1$; goto fim;

fe7: $j 7=j 7+1$; goto fim;

fe8: $j 8=j 8+1$; goto $f i m$;

fe9: $j 9=j 9+1$;

fim:

end ;

$n=j 1+j 2+j 3+j 4+j 5+j 6+j 7+j 8+j 9$;

$a 1=j 1 / / j 2 / / j 3$;

$a 2=j 4 / / j 5 / / j 6$;

$a 3=j 7 / / j 8 / / j 9$; 
$a=a 1|| a 2|| a 3 ;$

print a $\mathrm{n}$;

$\operatorname{arq} 1 d=e 1|| b 1|| x 1|| y 1$;

$\operatorname{arq} 2 d=e 2|| b 2|| x 2|| y 2$;

$\operatorname{arq} 3 d=e 3|| b 3|| x 3|| y 3 ;$

$\operatorname{arq} 7 d=e 7|| b 7|| x 7|| y 7$;

$\operatorname{arq} 8 \mathrm{~d}=e 8|| \mathrm{b} 8|| \mathrm{x} 8|| \mathrm{y} 8$;

arq9d=e9 | | b9 | |x9 | |y 9 ;

$\operatorname{arq} 10 d=e 10|| b 10|| x 10|| y 10$;

$\operatorname{arq} 14 d=e 14|| b 14|| x 14||$ y 14 ;

$\operatorname{arq} 15 d=e 15|| b 15|| x 15|| y 15$;

$\operatorname{arq} 16 d=e 16|| b 16|| x 16|| y 16$;

$\operatorname{arq} 17 \mathrm{~d}=\mathrm{e} 17|\mathrm{|b} 17||\mathrm{x} 17| \mid \mathrm{y} 17$;

$\operatorname{arq} 22 \mathrm{~d}=22||$ b22 | | x22 | | y 22 ;

$\operatorname{arq} 23 d=e 23|| b 23|| x 23||$ y 23 ;

$r 1=\{$ eg1 bn1 x1 $y 1\}$;

$r 2=\{$ eg2 bn2 x2 y2 $\}$;

$r 3=\{$ eg3 bn3 x3 y3 $\}$;

$r 7=\left\{\begin{array}{llll}\text { eg7 bn7 } & \mathrm{x} 7 & \mathrm{y} 7\end{array}\right\}$;

$r 8=\{$ eg8 bn8 $x 8$ y8 $\}$;

$r 9=\{$ eg9 bn9 $x 9$ y9 $\}$;

$\mathrm{r} 10=\{\mathrm{eg} 10$ bn10 $\mathrm{x} 10$ y10 $\}$;

$r 14=\{e g 14$ bn14 x14 y14 $\}$;

$\mathrm{r} 15=\{$ eg15 bn15 x15 y15 $\}$;

$\mathrm{r} 16=\{$ eg16 bn16 x16 y16 $\}$;

$r 17=\{e g 17$ bn17 $x 17$ y17 $\} ;$ 
r22=\{eg22 bn22 x22 y22\};

$r 23=\{$ eg23 bn23 x23 y23 $\}$;

create novo.arq1d from arq1d[colname=r1]; append from arq1d;

create novo.arq2d from arq2d[colname=r2]; append from arq2d;

create novo.arq3d from arq3d[colname=r3]; append from arq3d;

create novo.arq7d from arq7d[colname=r7]; append from arq7d;

create novo.arq8d from arq8d [colname=r8]; append from arq8d;

create novo.arq9d from arq9d[colname=r9]; append from arq9d;

create novo.arq10d from arq10d[colname=r10]; append from arq10d;

create novo.arq14d from arq14d[colname=r14]; append from arq14d;

create novo.arq15d from arq16d[colname=r15]; append from arq15d;

create novo.arq16d from arq16d[colname=r16]; append from arq16d;

create novo.arq17d from arq17d[colname=r17]; append from arq17d;

create novo.arq22d from arq22d[colname=r22]; append from arq22d;

create novo.arq23d from arq23d[colname=r23]; append from arq23d;

quit;

data novo.arq1d1; set novo.arq1d; if $\times 1=0$ then delete;

data novo.arq2d2; set novo.arq2d; if $x 2=0$ then delete;

data novo.arq3d3; set novo.arq3d; if $\times 3=0$ then delete;

data novo.arq7d7; set novo.arq7d; if $x 7=0$ then delete;

data novo.arq8d8; set novo.arq8d; if $x 8=0$ then delete;

data novo.arq9d9; set novo.arq9d; if $x 9=0$ then delete;

data novo.arq10d10; set novo.arq10d; if $\times 10=0$ then delete;

data novo.arq14d14; set novo.arq14d; if $x 14=0$ then delete;

data novo.arq15d15;

set novo.arq15d; 
if $\times 15=0$ then delete;

data novo.arq16d16; set novo.arq16d; if $x 16=0$ then delete;

data novo.arq17d17; set novo.arq17d; if $\mathrm{x} 17=0$ then delete;

data novo.arq22d22; set novo.arq22d; if $\mathrm{x} 22=0$ then delete;

data novo.arq23d23; set novo.arq23d; if $x 23=0$ then delete;

proc iml;

use novo.arq1d1; setin novo.arq1d1; $\operatorname{read}$ all $\operatorname{var}\{\operatorname{eg} 1 \mathrm{bn} 1 \mathrm{x} 1 \mathrm{y} 1\}$;

use novo.arq2d2; setin novo.arq2d2; $\operatorname{read}$ all $\operatorname{var}\{\operatorname{eg} 2$ bn2 $x 2$ y2\};

use novo.arq3d3; setin novo.arq3d3; $\operatorname{read}$ all $\operatorname{var}\{$ eg3 bn3 x3 y3\};

use novo.arq7d7; setin novo.arq7d7; $\operatorname{read}$ all $\operatorname{var}\{\operatorname{eg} 7$ bn7 $x 7$ y7\};

use novo.arq8d8; setin novo.arq8d8; $\operatorname{read}$ all var\{eg8 bn8 x8 y8\};

use novo.arq9d9; setin novo.arq9d9; read all var\{eg9 bn9 x9 y9\};

use novo.arq10d10; setin novo.arq10d10;

read all var $\{$ eg10 bn10 x10 y10\};

use novo.arq14d14; setin novo.arq14d14;

read all var\{eg14 bn14 x14 y14\};

use novo.arq15d15; setin novo.arq15d15;

read all var $\{$ eg15 bn15 x15 y15\};

use novo.arq16d16; setin novo.arq16d16;

read all var\{eg16 bn $16 \times 16$ y16\};

use novo.arq17d17; setin novo.arq17d17;

read all var $\{$ eg17 bn $17 \times 17$ y17\};

use novo.arq22d22; setin novo.arq22d22;

read all var $\{$ eg22 bn22 x22 y22\};

use novo.arq23d23; setin novo.arq23d23;

read all var $\{$ eg23 bn23 x23 y23\}; 
$\mathrm{m} 1=44 ; \mathrm{m} 2=17 ; \mathrm{m} 3=7 ; \mathrm{m} 7=14 ; \mathrm{m} 8=7 ; \mathrm{m} 9=3 ; \mathrm{m} 10=1 ; \mathrm{m} 14=2 ;$

$\mathrm{m} 15=1 ; \mathrm{m} 16=1 ; \mathrm{m} 17=1 ; \mathrm{m} 22=1 ; \mathrm{m} 23=1 ;$

xf1 $=\operatorname{shape}(0, m 1,1) ;$ yf $1=\operatorname{shape}(0, m 1,1) ; \operatorname{egf} 1=\operatorname{shape}(0, m 1,1)$;

bnf1=shape $(0, \mathrm{~m} 1,1)$;

$\mathrm{xf} 2=\operatorname{shape}(0, \mathrm{~m} 2,1) ; \operatorname{yf} 2=\operatorname{shape}(0, \mathrm{~m} 2,1) ; \operatorname{egf} 2=\operatorname{shape}(0, \mathrm{~m} 2,1)$;

bnf $2=\operatorname{shape}(0, \mathrm{~m} 2,1)$;

xf3 =shape $(0, m 3,1) ; y f 3=\operatorname{shape}(0, m 3,1) ; \operatorname{egf} 3=\operatorname{shape}(0, m 3,1)$;

bnf3=shape $(0, \mathrm{~m} 3,1)$;

$\operatorname{xf} 7=\operatorname{shape}(0, m 7,1) ; \operatorname{yf} 7=\operatorname{shape}(0, m 7,1) ; \operatorname{egf} 7=\operatorname{shape}(0, m 7,1)$;

bnf $7=\operatorname{shape}(0, m 7,1)$;

xf8 =shape $(0, m 8,1) ; y f 8=\operatorname{shape}(0, m 8,1) ; \operatorname{egf} 8=\operatorname{shape}(0, m 8,1)$;

bnf $8=\operatorname{shape}(0, \mathrm{~m} 8,1)$;

$\operatorname{xf} 9=\operatorname{shape}(0, m 9,1) ; \operatorname{yf} 9=\operatorname{shape}(0, m 9,1) ; \operatorname{egf} 9=\operatorname{shape}(0, m 9,1)$;

bnf $9=\operatorname{shape}(0, m 9,1)$;

$\operatorname{xf} 10=\operatorname{shape}(0, \mathrm{~m} 10,1) ; \operatorname{yf} 10=\operatorname{shape}(0, \mathrm{~m} 10,1) ; \operatorname{egf} 10=\operatorname{shape}(0, \mathrm{~m} 10,1) ;$

$\operatorname{bnf} 10=\operatorname{shape}(0, \mathrm{~m} 10,1)$;

$\operatorname{xf} 14=\operatorname{shape}(0, \mathrm{~m} 14,1) ; \operatorname{yf} 14=\operatorname{shape}(0, \mathrm{~m} 14,1) ; \operatorname{egf} 14=\operatorname{shape}(0, \mathrm{~m} 14,1) ;$

bnf 14=shape $(0, \mathrm{~m} 14,1)$;

$\operatorname{xf} 15=\operatorname{shape}(0, \mathrm{~m} 15,1) ; \operatorname{yf} 15=\operatorname{shape}(0, \mathrm{~m} 15,1) ; \operatorname{egf} 15=\operatorname{shape}(0, \mathrm{~m} 15,1)$;

bnf $15=\operatorname{shape}(0, \mathrm{~m} 15,1)$;

$\operatorname{xf} 16=\operatorname{shape}(0, \mathrm{~m} 16,1) ; \operatorname{yf} 16=\operatorname{shape}(0, \mathrm{~m} 16,1) ; \operatorname{egf} 16=\operatorname{shape}(0, \mathrm{~m} 16,1) ;$

bnf $16=\operatorname{shape}(0, \mathrm{~m} 16,1)$;

$\operatorname{xf} 17=\operatorname{shape}(0, \mathrm{~m} 17,1) ; \operatorname{yf} 17=\operatorname{shape}(0, \mathrm{~m} 17,1) ; \operatorname{egf} 17=\operatorname{shape}(0, \mathrm{~m} 17,1)$;

bnf $17=\operatorname{shape}(0, \mathrm{~m} 17,1)$;

$\operatorname{xf} 22=\operatorname{shape}(0, \mathrm{~m} 22,1) ; \operatorname{yf22}=\operatorname{shape}(0, \mathrm{~m} 22,1) ; \operatorname{egf} 22=\operatorname{shape}(0, \mathrm{~m} 22,1)$;

bnf22=shape $(0, \mathrm{~m} 22,1)$; 
$\operatorname{xf23}=\operatorname{shape}(0, \mathrm{~m} 23,1) ; \operatorname{yf23}=\operatorname{shape}(0, m 23,1) ; \operatorname{egf} 23=\operatorname{shape}(0, m 23,1) ;$

bnf23=shape $(0, \mathrm{~m} 23,1)$;

$j 1=0 ; \quad j 2=0 ; \quad j 3=0 ; \quad j 7=0 ; \quad j 8=0 ; \quad j 9=0 ; \quad j 10=0 ;$

$j 14=0 ; j 15=0 ; j 16=0 ; j 17=0 ; j 22=0 ; j 23=0$;

do $i=1$ to 132 by 3 ;

$\mathrm{j} 1=\mathrm{j} 1+1$

$x f 1[j 1]=x 1[i] ; \quad y f 1[j 1]=y 1[i] ;$

$\operatorname{egf} 1[j 1]=\operatorname{eg} 1[i] ; \operatorname{bnf} 1[j 1]=b n 1[i]$;

end ;

esc1=egf 1|| bnf 1||$x f 1|| y f 1$;

do $i=1$ to 323 by 19 ;

j2=j2+1;

$\operatorname{xf} 2[j 2]=x 2[i] ; \quad y f 2[j 2]=y 2[i] ;$

$\operatorname{egf} 2[j 2]=\operatorname{eg} 2[i] ; \operatorname{bnf} 2[j 2]=\operatorname{bn} 2[i]$;

end ;

esc2=egf 2|| bnf 2||$x f 2|| y f 2$;

do $i=1$ to 35 by 5 ;

$j 3=j 3+1$

$\operatorname{xf} 3[j 3]=x 3[i] ; \quad y f 3[j 3]=y 3[i] ;$

$\operatorname{egf} 3[j 3]=\operatorname{eg} 3[i] ; \operatorname{bnf} 3[j 3]=\operatorname{bn} 3[i]$;

end;

esc3=egf 3|| bnf 3||$x f 3||$ yf3;

do $i=1$ to 294 by 21 ;

$\mathrm{j} 7=\mathrm{j} 7+1$;

$\operatorname{xf} 7[j 7]=x 7[i] ; \quad y f 7[j 7]=y 7[i] ;$

$\operatorname{egf} 7[j 7]=\operatorname{eg} 7[i] ; \operatorname{bnf} 7[j 7]=\operatorname{bn} 7[i]$; 
end ;

esc7=egf7 | | bnf7 | |xf7 | |yf7;

do $i=1$ to 91 by 13 ;

$j 8=j 8+1$;

$x f 8[j 8]=x 8[i] ; \quad y f 8[j 8]=y 8[i] ;$

$\operatorname{egf} 8[j 8]=\operatorname{eg} 8[i] ; \operatorname{bnf} 8[j 8]=\operatorname{bn} 8[i]$;

end;

esc8=egf 8 | |bnf8 | |xf8 | |yf8;

do $i=1$ to 30 by 10 ;

j9=j9+1;

$\operatorname{xf} 9[j 9]=x 9[i] ; \quad y f 9[j 9]=y 9[i] ;$

$\operatorname{egf} 9[j 9]=\operatorname{eg} 9[i] ; \operatorname{bnf} 9[j 9]=\operatorname{bn} 9[i]$;

end;

esc9=egf 9|| bnf 9||$x f 9||$ yf 9 ;

do $i=1$ to 16 by 16 ;

$j 10=j 10+1$;

$\operatorname{xf} 10[j 10]=x 10[i] ; \quad \operatorname{yf} 10[j 10]=y 10[i] ;$

$\operatorname{egf} 10[j 10]=\operatorname{eg} 10[i] ; \operatorname{bnf} 10[j 10]=\operatorname{bn} 10[i]$;

end ;

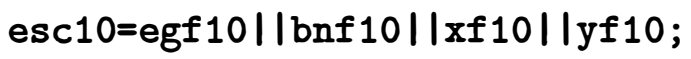

do $i=1$ to 14 by 7 ;

$j 14=j 14+1$;

$\operatorname{xf} 14[j 14]=x 14[i] ; \quad \operatorname{yf} 14[j 14]=y 14[i] ;$

$\operatorname{egf} 14[j 14]=\operatorname{eg} 14[i] ; \operatorname{bnf} 14[j 14]=\operatorname{bn} 14[i]$;

end;

esc14=egf 14 | | bnf14 | |xf14 | |yf 14; 
do $i=1$ to 22 by 22 ;

$j 15=j 15+1$;

$\operatorname{xf} 15[j 15]=x 15[i] ; \quad \operatorname{yf} 15[j 15]=y 15[i] ;$

$\operatorname{egf} 15[j 15]=\operatorname{eg} 15[i] ; \operatorname{bnf} 15[j 15]=\operatorname{bn} 15[i]$;

end;

esc15=egf 15|| bnf 15||$x f 15||$ yf 15 ;

do $i=1$ to 2 by 2 ;

$j 16=j 16+1$;

$\operatorname{xf} 16[j 16]=x 16[i] ; \quad y f 16[j 16]=y 16[i] ;$

$\operatorname{egf} 16[j 16]=\operatorname{eg} 16[i] ; \operatorname{bnf} 16[j 16]=\operatorname{bn} 16[i]$;

end;

esc16=egf 16|| bnf 16|| xf 16|| yf 16 ;

do $i=1$ to 4 by 4 ;

$j 17=j 17+1$;

$\operatorname{xf} 17[j 17]=x 17[i] ; \quad y f 17[j 17]=y 17[i] ;$

$\operatorname{egf} 17[j 17]=\operatorname{eg} 17[i] ; \operatorname{bnf} 17[j 17]=\operatorname{bn} 17[i]$;

end;

esc17=egf 17|| bnf 17|| xf 17|| yf 17 ;

do $i=1$ to 3 by 3 ;

$j 22=j 22+1$;

$\operatorname{xf22}[j 22]=x 22[i] ; \quad y f 22[j 22]=y 22[i]$;

$\operatorname{egf} 22[j 22]=\operatorname{eg} 22[i] ; \operatorname{bnf} 22[j 22]=\operatorname{bn} 22[i]$;

end;

esc22=egf 22|| bnf 22||$x f 22|| y f 22$;

do $i=1$ to 2 by 2 ;

$j 23=j 23+1$; 
$x f 23[j 23]=x 23[i] ; y f 23[j 23]=y 23[i] ;$

$\operatorname{egf} 23[j 23]=\operatorname{eg} 23[i] ; \operatorname{bnf} 23[j 23]=b n 23[i]$;

end;

esc23=egf 23|| bnf23 $\mid$ xf 23|| yf23;

esc $=$ esc $1 / /$ esc $2 / /$ esc $3 / /$ esc $7 / /$ esc $8 / /$ esc $9 / /$ esc $10 / /$ esc $11 / /$ esc14

//esc15//esc16//esc17//esc22//esc23;

$r 1=\left\{\begin{array}{llll}e g & b & y\end{array}\right\}$;

create novo.esc from esc [colname=r1];

append from esc;

proc print data=novo.esc; run;

proc iml;

use novo.esc;

setin novo.esc;

read all var\{eg bn $x \mathrm{y}\}$;

$\mathrm{m} 1=\operatorname{shape}(0, \operatorname{nrow}(x), 1)$; cens $1=\operatorname{shape}(0, \operatorname{nrow}(x), 1)$;

do $i=1$ to $\operatorname{nrow}(x)$;

$m 1[i]=\max (x[i], y[i])$;

if $(x[i]<y[i])$ then cens $1[i]=1$;

if $(x[i]>y[i])$ then cens $1[i]=0$;

end;

$n x b=\operatorname{sum}(x) ; n y b=\operatorname{sum}(y) ; r m=s u m(m 1) ; r=s u m(b n)$;

arq15=nrow( $x)|| r|| n x b|| n y b|| r m$;

$r=\{n \quad n \times b$ nyb $r m\}$;

create novo.arq15 from arq15 [colname=r];

append from arq15;

quit; 


\section{Programa 2}

Este programa implementado no "software" SAS é utilizado para calcular os estimadores de máxima verossimilhança para o modelo de BLOCK \& BASU através do método iterativo de Newton-Raphson.

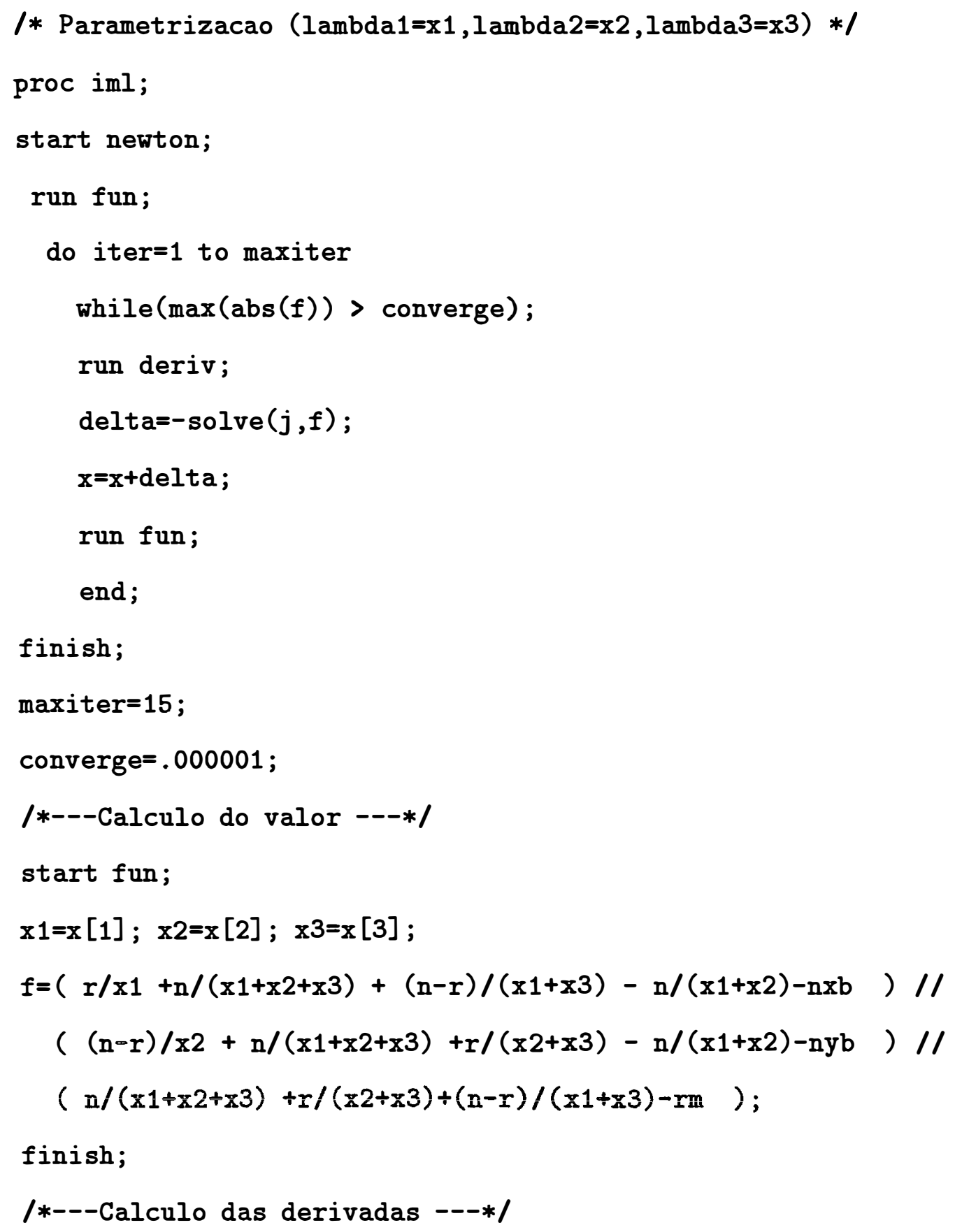


start deriv;

$$
\begin{aligned}
j= & ((-1 * r / x 1 * * 2-n /(x 1+x 2+x 3) * * 2-(n-r) /(x 1+x 3) * * 2 \\
& +n /(x 1+x 2) * * 2) \\
& \|(-n /(x 1+x 2+x 3) * * 2+n /(x 1+x 2) * * 2) \\
& \|(-n /(x 1+x 2+x 3) * * 2-(n-r) /(x 1+x 3) * * 2)) / / \\
& ((-n /(x 1+x 2+x 3) * * 2+n /(x 1+x 2) * * 2) \\
& \|(-1 *(n-r) / x 2 * * 2-n /(x 1+x 2+x 3) * * 2-r /(x 2+x 3) * * 2+ \\
n /(x 1+x 2) * * 2) & \\
& \|(-n /(x 1+x 2+x 3) * * 2-r /(x 2+x 3) * * 2)) / / \\
& ((-n /(x 1+x 2+x 3) * * 2-(n-r) /(x 1+x 3) * * 2) \\
& \|(-n /(x 1+x 2+x 3) * * 2-r /(x 2+x 3) * * 2) \\
& \|(-n /(x 1+x 2+x 3) * * 2-r /(x 2+x 3) * * 2-(n-r) /(x 1+x 3) * * 2)) ;
\end{aligned}
$$

\section{finish;}

do;

print "Solucao ", ;

use novo.arq15;

setin novo.arq15;

read all $\operatorname{var}\{n \mathrm{r}$ nxb nyb $\mathrm{rm}\}$;

/*---- Chute Inicial ---*/

$$
x=\{.04, .03, .03\} \text {; }
$$

chute $=\{.03, .03, .03\}$;

run newton;

$\mathrm{xt}=\mathrm{x}^{\prime}$;

print $x t$ iter;

end;

quit; 
Apresentam-se a seguir alguns programas computacionais implementados no "software": "SAS" utilizados para a construção do conjunto de dados necessários para a construção dos gráficos das verossimilhanças perfiladas unidimensionais e para os contornos das verossimilhanças perfiladas bidimensionais.

\section{Programa 3}

Este programa é implementado para construir o conjunto de dados necessários para a construção do gráfico da verossimilhança perfilada para o parâmetro $\lambda_{1}$ considerando-se o modelo de BLOCK \& BASU. Fixando-se $\lambda_{1}$ em um intervalo conveniente encontra-se os valores para $\lambda_{2}$ e $\lambda_{3}$ que maximizam o logaritmo da função de verossimilança (3.3). Com os valores $\left(\lambda_{1}, \hat{\lambda_{2}}\left(\lambda_{1}\right), \hat{\lambda_{3}}\left(\lambda_{1}\right)\right)$ obtidos calcula-se o valor assumido pela função de log-verossimilhança (3.3). O gráfico da verossimilhança perfilada para $\lambda_{1}$ é apresentado na Figura 9.2. Para construção de qualquer outro gráfico de verossimilhança perfilada unidimensional em qualquer outra reparametrização são necessárias modificações convenientes neste programa.

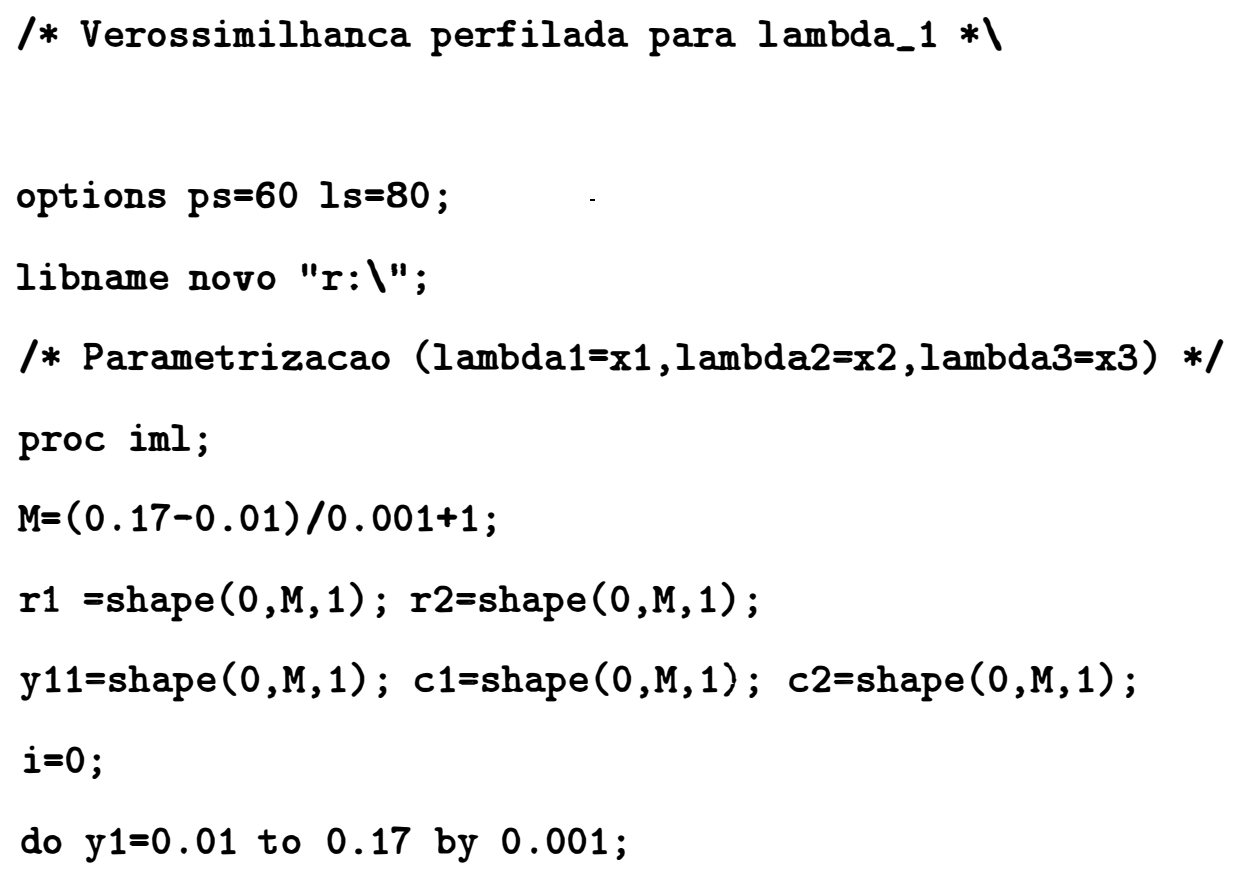


$i=i+1$;

start newton;

run fun;

do iter=1 to maxiter

while $(\max (\operatorname{abs}(f))>$ converge $)$;

run deriv;

delta $=-\operatorname{solve}(j, f)$;

$\mathrm{x}=\mathrm{x}+$ delt $\mathrm{a}$;

run fun;

end;

finish;

maxiter=15;

converge $=.000001$;

/*---Calculo do valor ---*/

start fun;

$x 2=x[1] ; x 3=x[2] ;$

$f=$

$$
\begin{aligned}
& ((n-r) / x 2+n /(x 1+x 2+x 3)+r /(x 2+x 3)-n /(x 1+x 2)-n y b) / / \\
& (n /(x 1+x 2+x 3)+r /(x 2+x 3)+(n-r) /(x 1+x 3)-r m) ;
\end{aligned}
$$

finish;

/*---Calculo das derivadas ---*/

start deriv;

$j=$

$(-1 *(n-r) / x 2 * * 2-n /(x 1+x 2+x 3) * * 2-r /(x 2+x 3) * * 2+$

$\mathrm{n} /(\mathrm{x} 1+\mathrm{x} 2) * * 2)$

|| $(-\mathrm{n} /(\mathrm{x} 1+\mathrm{x} 2+\mathrm{x} 3) * * 2-\mathrm{r} /(\mathrm{x} 2+\mathrm{x} 3) * * 2)) / /$ 


$$
\begin{aligned}
& (-n /(x 1+x 2+x 3) * * 2-r /(x 2+x 3) * * 2) \\
& \text { II }(-n /(x 1+x 2+x 3) * * 2-r /(x 2+x 3) * * 2-(n-r) /(x 1+x 3) * * 2)) ;
\end{aligned}
$$

finish;

do;

/* print "Solucao " ,; */

$x 1=y 1 ; n=100 ; \quad r=71 ; n x b=674.4523 ; n y b=1118.8833 ; r m=1343.6543 ;$

|*---- Chute Inicial ---*/

if $i=1$ then $x=\{.03, .03\}$; else $x=x$;

chute=x;

run newton;

$\mathrm{xt}=\mathrm{x}^{\prime}$;

/* print xt iter; */

end;

$r t=x t$;

$y 11[i]=y 1 ; r 1[i]=r t[1] ; r 2[i]=r t[2] ; c 1[i]=$ chute[1];c2[i]=chute[2];

/* print y1 rt chute; */

end;

$c=c 1|| c 2|| c 3 ; r=r 1|| r 2$;

$\operatorname{arq} 1=\mathrm{y} 11|| c|| r$;

$r=\{$ llbd1 chute1 chute2 emv1 emv2 $\}$;

create novo.arq1 from arq1 [colname=r];

append from arq1;

quit;

options ps $=5000$; 
proc iml;

use novo.arq1;

setin novo.arq1;

read all var\{vlbd1 chute1 chute2 emv1 emv2\};

$\mathrm{n}=100 ; \mathrm{r}=71 ; \mathrm{nxb}=674.45 ; \mathrm{nyb}=1118.88 ; \mathrm{Rm}=1343.65$;

$\mathrm{x} 1=\mathrm{vlbd} 1 ; \mathrm{x} 2=\mathrm{emv} 1 ; \mathrm{x} 3=\mathrm{emv} 2$;

$\operatorname{logver}=r * \log (x 1)+n * \log (x 1+x 2+x 3)+(n-r) * \log (x 2)+r * \log (x 2+x 3)+$

$(n-r) * \log (x 1+x 3)-x 1 * n x b-x 2 * n y b-x 3 * r m-n * \log (x 1+x 2)$;

$x 1$ est $=0.107818 ; x 2 e s t=0.048958 ; x 3 e s t=0.0533960$;

$\log v e r 1=r * \log (x 1$ est $)+n * \log (x 1$ est $+x 2$ est $+x 3 e s t)+(n-r) * \log (x 2$ est $)$

$+r * \log (x 2$ est $+x 3 e s t)+(n-r) * \log (x 1$ est $+x 3 e s t)-x 1$ est $* n x b-$

$x 2 e s t * n y b-x 3 e s t * r m-n * \log (x 1$ est $+x 2 e s t)$;

ver $=\exp ($ logver $) / \exp ($ logver 1$)$;

print $x 1$ ver;

quit ;

\section{Programa 4}

Este programa é implementado para construção do conjunto de dados necessários para construir o contorno da verossimilhança perfilada para os parâmetros $\lambda_{1}$ e $\lambda_{2}$ considerando-se o modelo de BLOCK \& BASU. Fixando-se $\lambda_{1}$ e $\lambda_{2}$ em intervalos convenientes encontra-se o valor para $\lambda_{3}$ que maximiza a função de log-verossimilança (3.3). Com os valores $\left(\lambda_{1}, \lambda_{2}, \hat{\lambda_{3}}\left(\lambda_{1}, \lambda_{2}\right)\right)$ obtidos calcula-se o valor assumido pela função de log-verossimilhança (3.3) e através de uma padronização conveniente constrói-se o contorno da verossimilhança perfilada para $\lambda_{1} \mathrm{e}$ $\lambda_{2}$ (Figura 9.4). Para construção de qualquer outro contorno em qualquer outra reparametrização são necessárias modificações convenientes neste programa. 
/* Verossimilhanca perfilada para lambda_1 e lambda_2 */

options ps=500 1s $=80$;

libname novo " $r: \backslash$ ";

I* Parametrizacao ( 1 ambda $1=x 1,1$ ambda2 $=x 2, \operatorname{lambda} 3=x 3$ ) */

proc iml;

M1 $=\operatorname{int}((0.2-0.01) / 0.01+1) ;$ M2=int $((0.08-0.001) / 0.01+1)$;

$\mathrm{m}=\mathrm{m} 1 * \mathrm{~m} 2$;

$r 1=\operatorname{shape}(0, M, 1) ; r 2=\operatorname{shape}(0, M, 1)$;

$\mathrm{y} 11=\operatorname{shape}(0, M, 1) ; c 1=\operatorname{shape}(0, M, 1) ; c 2=\operatorname{shape}(0, M, 1)$;

y22=shape $(0, M, 1)$;

$i=0$;

do $\mathrm{y} 1=0.01$ to 0.2 by 0.01 ;

do $y 2=0.001$ to 0.08 by 0.01 ;

$i=i+1$

print $i$ y1 y2;

start newton;

run fun;

do iter=1 to maxiter

while $(\max (\operatorname{abs}(f))>$ converge $)$;

run deriv;

$\operatorname{delta}=-\operatorname{solve}(j, f)$;

$x=x+$ delt $a$;

run fun;

end;

finish; 
maxiter=15;

converge $=.000001$;

/*---Calculo do valor ---*/

start fun;

$\mathrm{x} 3=\mathrm{x}$;

$f=(n /(x 1+x 2+x 3)+r /(x 2+x 3)+(n-r) /(x 1+x 3)-r m) ;$

finish;

/*---Calculo das derivadas ---*/

start deriv;

$j=(-n /(x 1+x 2+x 3) * * 2-r /(x 2+x 3) * * 2-(n-r) /(x 1+x 3) * * 2) ;$

finish;

do;

print "Solucao", ;

$\mathrm{x} 1=\mathrm{y} 1 ; \mathrm{x} 2=\mathrm{y} 2 ; \mathrm{n}=100 ; \mathrm{r}=71 ; \mathrm{nxb}=674.4523 ;$

$\mathrm{nyb}=1118.8833 ; \mathrm{rm}=1343.6543$;

/*---- Chute Inicial ---*/

if $i=1$ then $x=\{.03\}$; else $x=x$;

chute=x;

run newton;

print $x$ iter;

end;

$\mathrm{y} 11[\mathrm{i}]=\mathrm{y} 1 ; \mathrm{r} 1[\mathrm{i}]=\mathrm{x} ; \quad \mathrm{c} 1[\mathrm{i}]=$ chute $[1]$;

y22 [i] $=y 2$;

print y1 y2 $x$ chute;

end ;

end; 
$\mathrm{c}=\mathrm{c} 1 ; \mathrm{r}=\mathrm{r} 1$;

$\operatorname{arq} 1=y 11|| y 22|| c|| r$;

$r=\left\{v_{-} l b d_{-} 1 v_{-} l b d_{-} 2\right.$ chute $e_{-1}$ emv_1 $\}$;

create novo.arq1 from arq1 [colname=r];

append from arq1;

quit ;

options ps=200 ls=80;

proc print data=novo.arq1; run;

proc print data=novo.arq1; run;

\section{Programa 5}

Apresenta-se a seguir um programa implementado para construção do "t-plot" para $\lambda_{1}$ (Figura 9.3). Para construção de qualquer outro modificacões convenientes deverão ser feitas.

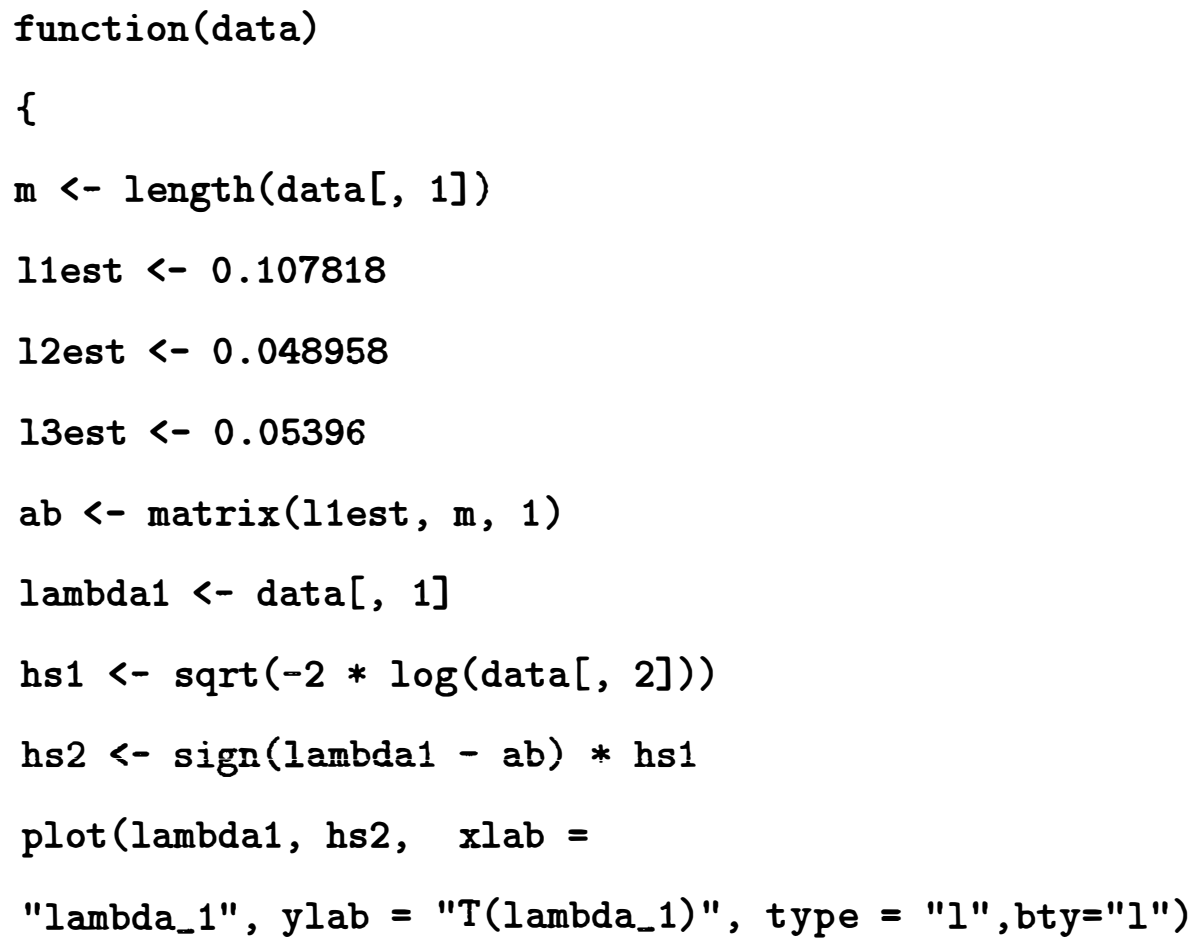




\section{Programa 6}

Este programa é implementado para construir a verossimilhança perfilada para $\theta_{0}$ quando considera-se a reparametrização Guerrero \& Johnson. Devido a complexidade das derivadas de primeira e segundas ordens não é utilizado o método iterativo de Newton-Raphson e sim um método de otimização não linear no qual as derivadas são calculadas por diferenças finitas assim utiliza-se a rotina NLPNRR disponível no "software" SAS.

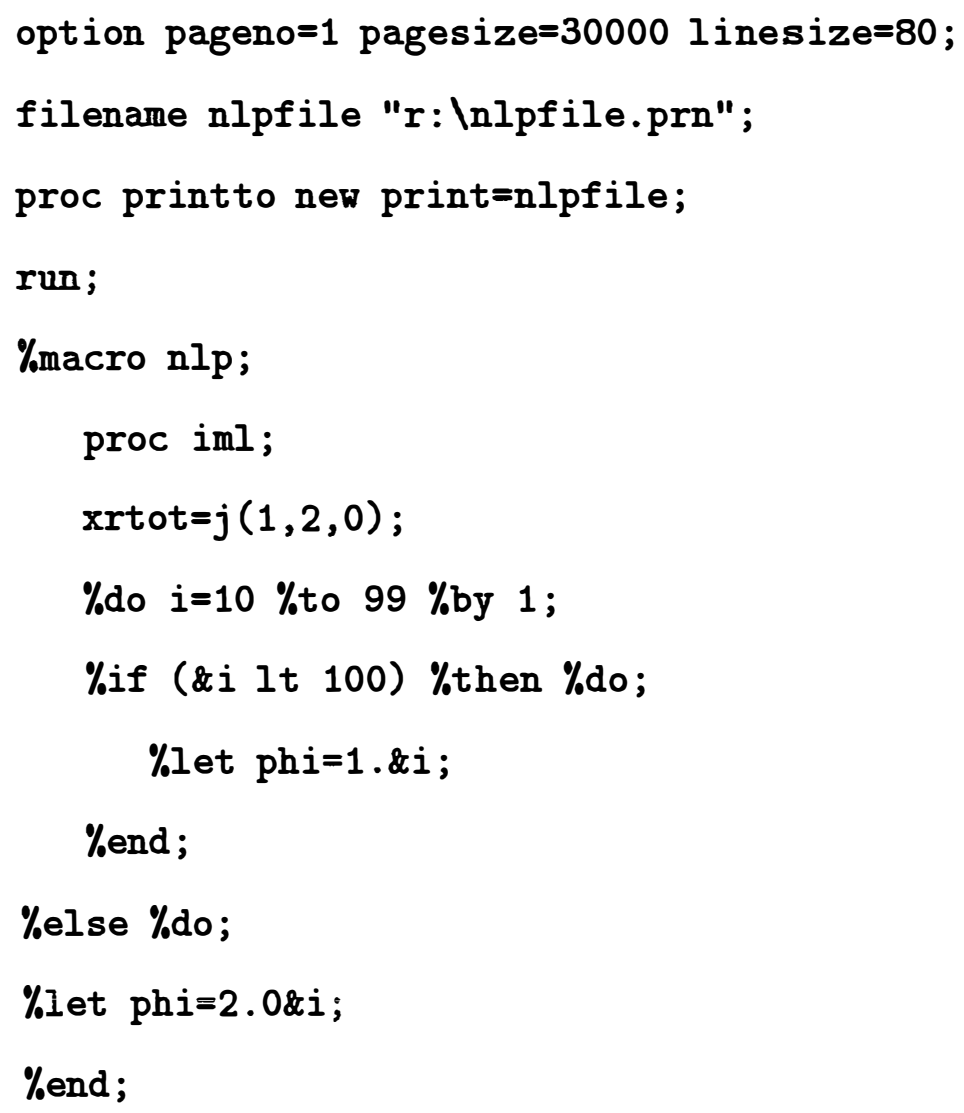


/* Definindo a funcao a ser maximizada */

start f_myf\&i(x);

/* Para os tempos $1,2,3,5,10$ basta trocar a variacao de phi */

/* e os valores de to e xi */

$/ *$ Se to $=1 \mathrm{xi}=0.6 * /$

$/ *$ Se t $0=2 x i=0.8 * /$

$/ *$ Se $t 0=3 x i=0.8 * /$

$/ *$ Se t $0=5 \mathrm{xi}=0.6 * 1$

/* Se t $0=10 \mathrm{xi}=0.6 * /$

$\mathrm{n}=100 ; \mathrm{r}=71 ; \mathrm{nxb}=674.4523 ; \mathrm{nyb}=1118.8833$;

$\mathrm{Rm}=1343.6543 ; \mathrm{t} 0=1 ; \mathrm{xi}=0.6$;

phi=\&phi;

$\mathrm{p} 11=\exp ((1 / \mathrm{xi}) * \log (1+\mathrm{xi} * \mathrm{ph} i)) /(1+\exp ((1 / \mathrm{xi}) * \log (1+\mathrm{x} i * \mathrm{ph} i)))$;

$\mathrm{p} 1=\log (\mathrm{p} 11) / \mathrm{t} 0$;

/* Verossimilhanca perfilada para theta_0*/

$f=r * \log (x[1])+n * \log (-p 1)+(n-r) * \log (x[2])+r * \log (-p 1-x[1])$

$+(n-r) * \log (-p 1-x[2])-n * \log (x[1]+x[2])+(-x[1] * n x b-x[2] * n y b$

$-(-\mathrm{p} 1-\mathrm{x}[1]-\mathrm{x}[2]) * \mathrm{Rm})$;

$\operatorname{return}(f)$;

finish f_myf\&i;

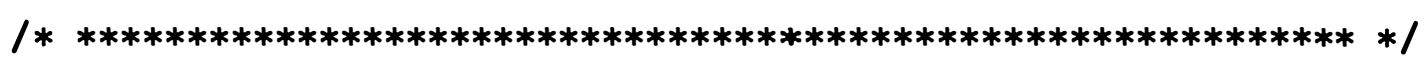

$x=\left\{\begin{array}{lll}0.10 & 0.04\end{array}\right\}$

optn $=\{12\} ; / *$ Quero maximizar a funcao portanto optn $[1]=1 * /$

call NLPNRR(rc,xr,"f_myf\&i", x,optn);

phi=\&phi ; 
$\%$ if (\&i eq 10 ) \%then \%do;

phitot=\&phi;

xrtot $=x r$;

\%end;

$\%$ else \%do;

phitot=phitot//phi;

xrtot=xrtot//xr;

$\%$ end;

\%end;

xrtot=phitot $\mid$ |xrtot;

xrnames=\{"phi" "x1" "x2" $\}$;

create xrtot from xrtot [colname=xrnames];

append from xrtot;

\%mend nlp;

quit;

$\%$ nlp;

proc printto;

run;

* Extract values of objective function from output;

data objfunct (keep=objfunct);

length objfunct $\$ 12$;

retain nobj 0 ;

infile nlpfile lrecl=80 pad missover;

input Q2 var \$char79.;

if (index(var, "Value of Objective Function $="$ ) ne 0 ) then do; objfunct=input ( $\operatorname{scan}(\operatorname{var}, 6, "$ ") , 9.3); 
nobj=nobj+1;

if $\bmod (n o b j, 2)$ eq 0 then output objfunct;

end;

run;

* Merge objective function values with parameter values;

data both;

merge xrtot objfunct;

output both;

run;

proc print data=both;

run;

\section{Programa 7}

Neste programa apresenta-se um programa computacional implementado no "software" MAPLE V para calcular a medida STD quando considera-se a reparametrização natural para o modelo de BLOCK \& BASU $\left(\mu_{1}, \mu_{2}, \phi\right)$ apresentada na Secção 5.5 .

\section{Programa 7.1}

Construção da matriz $\mathrm{D}$ de terceiras derivadas ne :cessárias para o cálculo da medida STD (matd).

$>\mathrm{L}:=\left(\operatorname{lambda}_{1}\right]^{\wedge} \mathrm{r} * 1123^{\wedge} \mathrm{n} * 1 \mathrm{ambda}[2]^{-}(\mathrm{n}-\mathrm{r}) * 123^{\wedge} \mathrm{r} *$

$\left.>113^{\wedge}(n-r)\right) / 112^{\wedge} n * \exp (-1 a m b d a[1] * n \times b-l a m b d a[2] * n y b-l a m b d a[3] * R m)$;

$>$ lv:=simplify $(\log (L))$ : 
$>\# \mathrm{k}[0]=1123$

$>k[0]:=(-\log ($ theta $[0])) / t[0]:$

$>\quad \operatorname{aa}[1]:=\operatorname{mu}[1] * \mathrm{k}[0]-1:$

$>\quad \mathrm{aa}[2]:=\operatorname{mu}[2] * \mathrm{k}[0]-1$ :

$>\quad b b[1]:=m u[1]-m u[2]:$

> 1123:=lambda[1]+lambda[2]+lambda [3] :

> 123:=lambda[2]+lambda[3] :

> 113:=lambda[1]+lambda[3]:

> 112:=lambda[1]+lambda[2] :

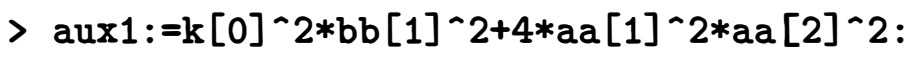

$>\operatorname{lambda}[2]:=\mathrm{k}[0]-((\mathrm{k}[0] * \mathrm{bb}[1]-2 * \mathrm{aa}[1] * \mathrm{aa}[2])$

$>+\operatorname{sqrt}(\operatorname{aux} 1)) /(2 * a a[1] * b b[1])):$

$>\operatorname{lambda}[1]:=\operatorname{lambda}[2] *(\mathrm{k}[0] /((\mathrm{k}[0]-\operatorname{lambda}[2]) * a a[1])-1):$

$>\operatorname{lambda}[3]:=\mathrm{k}[0]-1 \mathrm{ambda}[1]-1 \mathrm{ambda}[2]$ :

$>\operatorname{theta}[0]:=(x i * \operatorname{ph} i+1) \wedge(1 / x i) /((x i * \operatorname{ph} i+1) \wedge(1 / x i)+1):$

$>$ lv:

> func: $=\operatorname{subs}(\mathrm{n}=100, \mathrm{r}=71, \mathrm{Rm}=1343.65, \mathrm{nxb}=674.45, \mathrm{nyb}=1118.88, \mathrm{lv})$ :

> Calculo da terceiras derivadas \#

> sd121:=diff(func,phi,mu[1],phi);

$>\operatorname{with}($ linalg):

$>$ \# $\mathrm{tp}:=$ vector $([1,2,3,5,10,20,30])$;

> \#xxi:=vector $([-1,-0.8,-0.6,-0.4,-0.2,0.2,0.4,0.6,0.8,1])$;

$>$ for $i$ from 1 to 7 do:

$>$ for $j$ from 1 to 10 do:

$>$ theta $[0]:=\exp (-(0.107818+0.048958+0.053960) * \operatorname{tp}[i])$;

$>\operatorname{phic}:=\left((\operatorname{theta}[0] /(1-\operatorname{theta}[0]))^{\wedge} \operatorname{xxi}[j]-1\right) / \operatorname{xxi}[j]$; 
$>d:=\operatorname{simplify}(\operatorname{subs}(t[0]=\operatorname{tp}[i], x i=x x i[j], \operatorname{phi}=\operatorname{phic}, \operatorname{mu}[1]=6.67$, $\mathrm{mu}[2]=11.43, \mathrm{sd} 121)$ );

$>\mathrm{v} 1:=\operatorname{array}(1, .5):$

$>\mathrm{v} 1[1]:=\operatorname{tp}[i]: \mathrm{v} 1[2]:=\operatorname{xxi}[j]: \quad \mathrm{v} 1[3]:=\operatorname{theta}[0]:$

$>$ v1 $[4]:=$ phic: $v 1[5]:=d:$

$>\operatorname{latex}(v 1):$

$>$ od:

$>$ od:

\section{Programa 7.2}

Programa para o cálculo da medida STD.

> \#alculo de STD \#

$>\operatorname{lv} 1:=r * \operatorname{lambda}[1]+n * \log (1123)+(n-r) * \log (\operatorname{lambda}[2])$

$$
+r * \log (123)+(n-r) * \log (113):
$$

$>\operatorname{lv} 2:=-n * \log (112)-\operatorname{lambda}[1] * n \times b-n y b * \operatorname{lambda}[2]-\operatorname{lambda}[3] * \mathrm{Rm}$ :

$>\operatorname{lv}:=\operatorname{lv} 1+\operatorname{lv} 2$;

$>\mathrm{k}[0]:=(-\log (\operatorname{theta}[0])) / \mathrm{t}[0]$ :

$>\mathrm{aa}[1]:=\operatorname{mu}[1] * \mathrm{k}[0]-1$ :

$>\mathrm{aa}[2]:=\mathrm{mu}[2] * \mathrm{k}[0]-1$ :

$>\mathrm{bb}[1]:=\mathrm{mu}[1]-\mathrm{mu}[2]$ :

$>1123:=1$ ambda $[1]+\operatorname{lambda}[2]+\operatorname{lambda}[3]:$

$>123:=1$ ambda [2]+lambda [3]:

$>113:=1$ ambda [1]+lambda [3]:

$>112:=1$ ambda $[1]+\operatorname{lambda}[2]$ :

$>\operatorname{aux} 1:=k[0]^{\wedge} 2 * b b[1]^{\wedge} 2+4 * a a[1]^{\wedge} 2 * a a[2]^{\wedge} 2$ :

$>\operatorname{lambda}[2]:=\mathrm{k}[0]-(((\mathrm{k}[0] * \mathrm{bb}[1]-2 * a a[1] * a a[2])$ 
$+\operatorname{sqrt}(\operatorname{aux} 1)) /(2 * a a[1] * b b[1])):$

$>\operatorname{lambda}[1]:=\operatorname{lambda}[2] *(\mathrm{k}[0] /((\mathrm{k}[0]-\operatorname{lambda}[2]) * a a[1])-1):$

$>\operatorname{lambda}[3]:=\mathrm{k}[0]-1 \mathrm{ambda}[1]-\operatorname{lambda}[2]$ :

$>\operatorname{theta}[0]:=(x i * \operatorname{ph} i+1)^{\wedge}(1 / x i) /\left((x i * \operatorname{ph} i+1)^{\wedge}(1 / x i)+1\right):$

$>$ func: $=\operatorname{subs}(\mathrm{n}=100, \mathrm{r}=71, \mathrm{Rm}=1343.65, \mathrm{nxb}=674.45, \mathrm{nyb}=1118.88, \mathrm{lv}):$

$>\operatorname{with}($ linalg):

$>\operatorname{readlib}($ readdata):

> 1:=readdata $(x x i a, 10):$

$>\mathrm{a}:=\operatorname{convert}(1, \operatorname{array})$ :

$>\operatorname{xxi}:=\operatorname{convert}(\mathrm{a}$, vector $)$ :

$>\# \operatorname{xxi}[70]$ :

> $\mathrm{m}:=\operatorname{readdata}($ tempo,10):

$>\mathrm{m} 1:=\operatorname{convert}(\mathrm{m}$, array $)$ :

$>$ tp:=convert (m1, vector):

> 11:=readdata (matda ,10):

$>$ matd:=convert (11, array):

> \#print (matd) :

$>\mathrm{v}:=\operatorname{array}(1 \ldots 3)$ :

>\#for $i$ from 1 to 70 do

$>\# v[1]:=i$ :

$>\# v[2]:=m 2[i]:$

$>\# v[3]:=x x i[i]:$

> \#latex(v):

$>$ \#od :

$>$ for $i$ from 1 to 5 do:

$>$ for $j$ from 1 to 5 do: 
$>$ theta $[0]:=\exp (-(0.107818+0.048958+0.053960) * \operatorname{tp}[i])$;

$>\operatorname{phic}:=\left((\text { theta }[0] /(1-\operatorname{theta}[0]))^{\wedge} \operatorname{xxi}[j]-1\right) / \operatorname{xxi}[j]:$

$>$ \# Construcao de b1.

$>$ b11:=diff (func,phi,phi):

$>\mathrm{b} 1[1,1]:=\operatorname{simplify}(\operatorname{subs}(\mathrm{ph} i=$ phic, $\operatorname{mu}[1]=6.67, \mathrm{mu}[2]=11.43$,

$\left.\left.>t[0]=t_{p}[i], \operatorname{xi}=\operatorname{xxi}[j], b 11\right)\right)$;

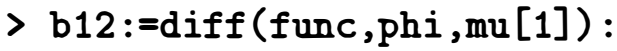

$>\mathrm{b} 1[1,2]:=\operatorname{simplify}(\operatorname{subs}(\mathrm{phi}=\mathrm{phic}, \mathrm{mu}[1]=6.67, \mathrm{mu}[2]=11.43$,

$>\quad t[0]=\operatorname{tp}[i], x i=x x i[j], b 12))$;

$>\mathrm{b} 13:=\operatorname{diff}($ func,phi,mu[2]):

$>\mathrm{b} 1[1,3]:=\operatorname{simplify}(\operatorname{subs}(\mathrm{phi}=\operatorname{phic}, \mathrm{mu}[1]=6.67, \mathrm{mu}[2]=11.43$,

$>t[0]=\operatorname{tp}[i], x i=x x i[j], b 13))$;

$>\mathrm{b} 22:=\operatorname{diff}(\mathrm{func}, \operatorname{mu}[1], \mathrm{mu}[1])$ :

$>\mathrm{b} 1[2,2]:=\operatorname{simplify}(\operatorname{subs}(\mathrm{phi}=\operatorname{phic}, \mathrm{mu}[1]=6.67, \mathrm{mu}[2]=11.43$,

> $t[0]=\operatorname{tp}[i], \operatorname{xi}=\operatorname{xxi}[j], \mathrm{b} 22))$;

$>\mathrm{b} 23:=\operatorname{diff}(\mathrm{func}, \mathrm{mu}[1], \mathrm{mu}[2])$ :

$>\mathrm{b} 1[2,3]:=\operatorname{simplify}(\operatorname{subs}(\mathrm{phi}=\operatorname{phic}, \mathrm{mu}[1]=6.67, \mathrm{mu}[2]=11.43$,

$>t[0]=\operatorname{tp}[i], \operatorname{xi}=\operatorname{xxi}[j], \mathrm{b} 23))$;

$>\mathrm{b} 33:=\operatorname{diff}(f u n c, m u[2], m u[2]):$

$>\mathrm{b} 1[3,3]:=\operatorname{simplify}(\operatorname{subs}(\mathrm{phi}=\mathrm{phic}, \mathrm{mu}[1]=6.67, \mathrm{mu}[2]=11.43$,

$>t[0]=\operatorname{tp}[i], \operatorname{xi}=\operatorname{xxi}[j], \mathrm{b} 33)$ ));

$>\mathrm{b} 1[2,1]:=\mathrm{b} 1[1,2]$ :

$>\mathrm{b} 1[3,1]:=\mathrm{b} 1[1,3]$ :

$>\mathrm{b} 1[3,2]:=\mathrm{b} 1[2,3]$ :

$>\mathrm{b} 11:=\operatorname{matrix}(3,3,[\mathrm{~b} 1[1,1], \mathrm{b} 1[1,2], \mathrm{b} 1[1,3], \mathrm{b} 1[1,2], \mathrm{b} 1[2,2]$,

$>\mathrm{b} 1[2,3], \mathrm{b} 1[1,3], \mathrm{b} 1[2,3], \mathrm{b} 1[3,3]])$ : 
$>\mathrm{b}:=$ inverse(b11): $\operatorname{print}(\mathrm{b})$;

$>d[1,1,1]:=\operatorname{matd}[i, 1]$;

$>d[1,2,1]:=\operatorname{matd}[i, 2]$;

$>d[1,3,1]:=\operatorname{matd}[i, 3]$;

$>d[2,2,1]:=\operatorname{matd}[i, 4]$;

$>d[2,3,1]:=\operatorname{matd}[i, 5]$;

$>d[3,3,1]:=\operatorname{matd}[i, 6]$;

$>d[2,2,2]:=\operatorname{matd}[i, 7]$;

$>d[2,3,2]:=\operatorname{matd}[i, 8]$;

$>d[3,3,2]:=\operatorname{matd}[i, 9]$;

$>d[3,3,3]:=\operatorname{matd}[i, 10]$;

$>\mathrm{d}[2,1,1]:=\mathrm{d}[1,2,1]: \mathrm{d}[3,1,1]:=\mathrm{d}[1,3,1]: \mathrm{d}[3,2,1]:=\mathrm{d}[2,3,1]:$

$>\mathrm{d}[1,1,2]:=\mathrm{d}[1,2,1]: \mathrm{d}[1,2,2]:=\mathrm{d}[2,2,1]: \mathrm{d}[1,3,2]:=\mathrm{d}[2,3,1]:$

$>\mathrm{d}[2,1,2]:=\mathrm{d}[2,2,1]:$

$>d[3,1,2]:=d[2,3,1]: d[3,2,2]:=d[2,3,2]:$

$>\mathrm{d}[1,1,3]:=\mathrm{d}[1,3,1]: \mathrm{d}[1,2,3]:=\mathrm{d}[2,3,1]: \mathrm{d}[1,3,3]:=\mathrm{d}[3,3,1]:$

$>d[2,1,3]:=d[2,3,1]: d[2,2,3]:=d[2,3,2]: d[2,3,3]:=d[3,3,2]:$

$>d[3,1,3]:=d[3,3,1]: d[3,2,3]:=d[3,3,2]$ :

$>$

$>$ std: $=0$ :

$>$ for $i 11$ from 1 to 3 do

$>$ for $j 11$ from 1 to 3 do

> for $k 11$ from 1 to 3 do

> for 111 from 1 to 3 do

$>$

for $m 11$ from 1 to 3 do for $\mathrm{n} 11$ from 1 to 3 do 
$>\quad \mathrm{std}:=\mathrm{b}[\mathrm{i} 11, \mathrm{j} 11] * \mathrm{~b}[\mathrm{l11}, \mathrm{m} 11] * \mathrm{~b}[\mathrm{k} 11, \mathrm{n} 11] * \mathrm{~d}[\mathrm{i} 11, \mathrm{j} 11, \mathrm{k} 11] *$

$>\quad \mathrm{d}[111, \mathrm{~m} 11, \mathrm{n} 11]+\mathrm{std}$ :

> od: od: od: od: od: od:

$>\mathrm{v} 1:=\operatorname{array}(1 \ldots 5): \mathrm{v} 2:=\operatorname{array}(1 \ldots 3):$

$>\mathrm{v} 1[1]:=\operatorname{tp}[\mathrm{i}]: \mathrm{v} 1[2]:=\operatorname{xxi}[\mathrm{j}]: \mathrm{v} 1[3]:=\operatorname{theta}[0]: \mathrm{v} 1[4]:=\operatorname{phic}: \mathrm{v} 1[5]:=\operatorname{std}:$

$>>\mathrm{i} 11:=0: \mathrm{j} 11:=0: \mathrm{k} 11:=0: 111:=0: \mathrm{m} 11:=0: \mathrm{n} 11:=0:$ phic $:=0:$

$>$ latex(v1): od: od:

\section{Programa 8}

Este programa é implementado para gerar a amostra G-MH isto é, para obter a amostra "Gibbs-com-Metropolis-Hastings" através da metodologia apresentada no Capítulo 8. Para obtenção de amostras G-MH necessárias para o desenvolvimento dos exemplos ilustrativos 2 e 3 modificações convenientes foram feitas no programa que segue.

/* k: \sas\regxy.sas */

/* PROGRAMA $01 *$ /

/* Este programa le os dados gerados */

/* Tamanho da amostra $\mathrm{n}=1000 \quad *$ /

libname novo "r:";

options pagesize $=50$;

/* BERNOULLI COM PARAMETRO $\mathrm{p}=11 /(11+12)$ bin $<-\mathrm{r}(1000,1, \mathrm{p}) * /$

data roseli;

infile "k: \sps\regeb.sps";

input obs eg bn 86 ;

1* EXPONENCIAL COM PARAMETRO $1 / 1,1=11+12+13$

$1=$ taxa de falha exp<-rexp $(1000,1) * I$ 
/* data roseli1;

infile "k: Isps\regexp.sps";

input eg QQ; *I

data novo.tese;

set roseli;

/* set roseli1; */

run;

/* PROGRAMA $02 *$ /

/* Calcula os valores para $\mathrm{x}$ e $\mathrm{y} * /$

proc iml worksize $=300$;

use novo.tese;

setin novo.tese;

read all var\{eg bn\};

$11=0.08 ; 12=0.06 ; 13=0.06 ;$

$112=11+12$;

$113=11+13$;

$123=12+13$;

$1=11+12+13$;

$y=\operatorname{shape}(0, \operatorname{nrow}(e g), 1)$;

$x=\operatorname{shape}(0, \operatorname{nrow}(e g), 1)$;

do $i=1$ to nrow(eg);

if $b n[i]=1$ then goto ar;

else goto er;

ar:

$x[i]=\operatorname{eg}[i]$; 
pyMx $=1 / 112 * \exp (-123 * x[i])-$

$13 / 112 * \exp (-1 * x[i])$;

EydyMx $=1 /(112 *$ pyMx $) *(x[i] *$

$\exp (-123 * x[i])+1 / 123 *$

$\exp (-123 * x[i])-13 / 1 * x[$

i] $* \exp (-1 * x[i])-13 / 1 * *$

$2 * \exp (-1 * x[i]))$;

$y[i]=$ EydyMx;

goto her;

er:

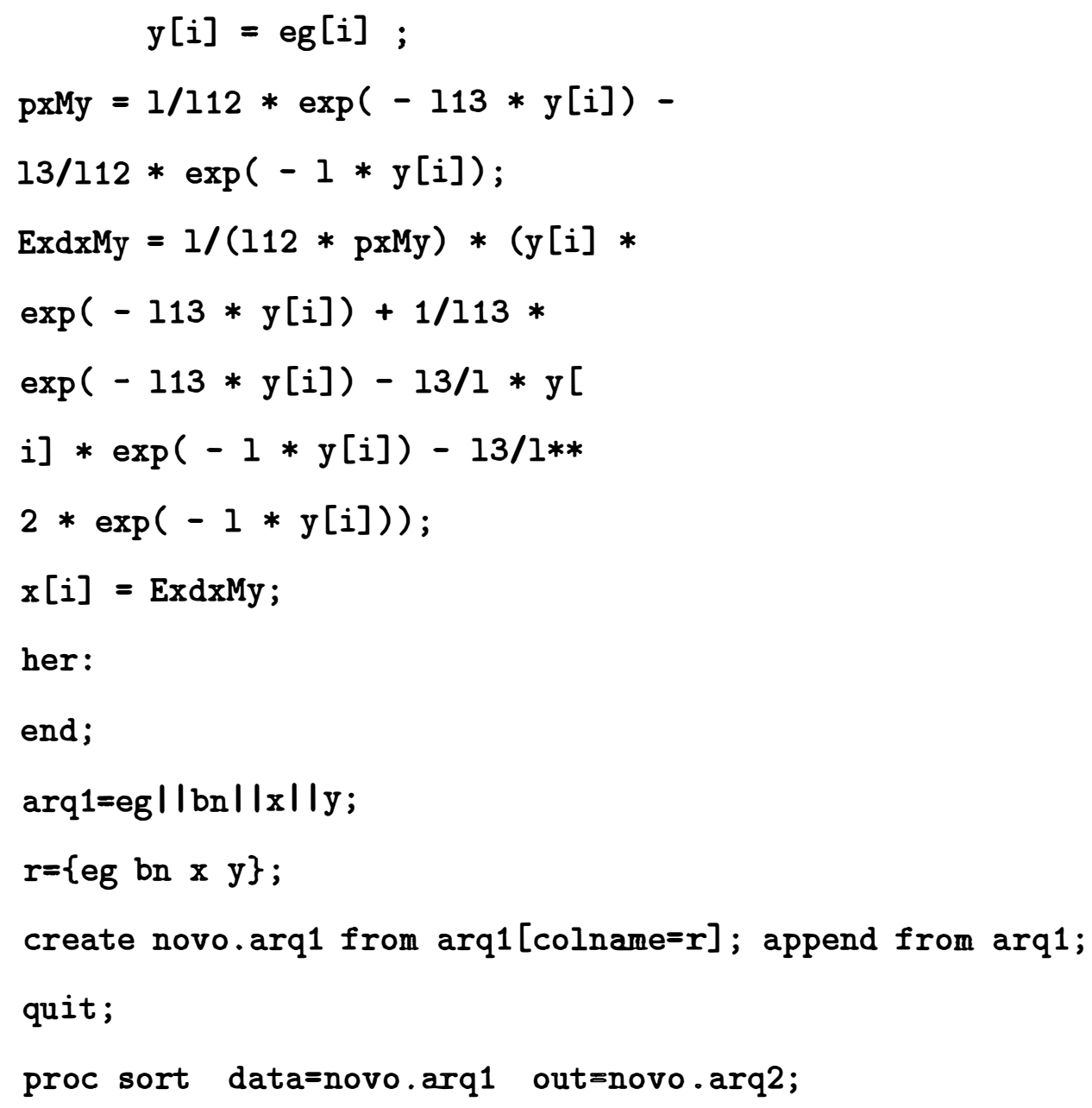


by $x$;

run;

proc print data=novo.arq1; run;

$x m=j(10,9,0) ; y m=j(10,9,0) ; \quad b m=j(10,9,0)$;

$\operatorname{xm}[, 1]=x[1: 10] ; \operatorname{ym}[, 1]=y[1: 10] ; \operatorname{bm}[, 1]=b n[1: 10]$;

$\operatorname{xm}[, 2]=x[11: 20] ; \operatorname{ym}[, 2]=y[11: 20] ; \operatorname{bm}[, 2]=b n[11: 20] ;$

$\operatorname{xm}[, 3]=x[21: 30] ; \operatorname{ym}[, 3]=y[21: 30] ; \operatorname{bm}[, 3]=b n[21: 30]$;

$\operatorname{xm}[, 4]=x[31: 40] ; \operatorname{ym}[, 4]=y[31: 40] ; \operatorname{bm}[, 4]=b n[31: 40]$;

$\operatorname{xm}[, 5]=x[41: 50] ; \operatorname{ym}[, 5]=y[41: 50] ; \operatorname{bm}[, 5]=b n[41: 50] ;$

$\operatorname{xm}[, 6]=x[51: 60] ; \operatorname{ym}[, 6]=y[51: 60] ; \operatorname{bm}[, 6]=b n[51: 60]$;

$\operatorname{xm}[, 7]=x[61: 70] ; \operatorname{ym}[, 7]=y[61: 70] ; \operatorname{bm}[, 7]=b n[61: 70]$;

$\operatorname{xm}[, 8]=x[71: 80] ; \operatorname{ym}[, 8]=y[71: 80] ; \operatorname{bm}[, 8]=b n[71: 80] ;$

$\operatorname{xm}[, 9]=x[81: 90] ; \operatorname{ym}[, 9]=y[81: 90] ; \operatorname{bm}[, 9]=b n[81: 90] ;$

$\mathrm{m} 1=\operatorname{shape}(0, \operatorname{nrow}(x), 1) ; \operatorname{cens} 1=\operatorname{shape}(0, \operatorname{nrow}(x), 1)$;

do $i=1$ to $\operatorname{nrow}(x)$;

$\mathrm{m} 1[\mathrm{i}]=\max (\mathrm{x}[\mathrm{i}], \mathrm{y}[\mathrm{i}])$;

if $(x[i]<y[i])$ then cens $1[i]=1$;

if $(x[i]>y[i])$ then $\operatorname{cens} 1[i]=0$;

end;

$\mathrm{nxb}=\operatorname{sum}(\mathrm{x})$;

nyb=sum $(y)$;

$\mathrm{rm}=\operatorname{sum}(\mathrm{m} 1)$;

$r=\operatorname{sum}(b n)$;

$\operatorname{arq15=nrow}(\mathrm{x})|| \mathrm{r}|\operatorname{nxb}||\mathrm{nyb}| \mid \mathrm{rm}$;

$\mathrm{r} 15=\{\mathrm{n} \mathrm{r} \times \mathrm{xb}$ nyb $\mathrm{rm}\}$; 
create novo.arq15 from arq15 [colname=r15];

append from arq15;

$\operatorname{arq} 20=x m|~| y m|~| b m$;

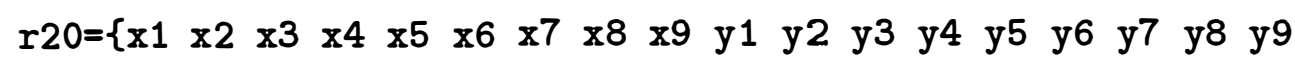

b1 b2 b3 b4 b5 b6 b7 b8 b9\};

create novo.arq20 from arq20 [colname=r20];

append from arq20;

quit;

proc print data=novo.arq20; run;

proc print data=novo.arq15; run;

proc iml;

use novo.arq20;

setin novo.arq20;

read all $\operatorname{var}\{x 1 \times 2 \times 3 \times 4 \times 5 \times 6 \times 7 \times 8$ x9 y1 y2 y3 y4 y5 y6 y7 y8 y9 b1 b2 b3 b4 b5 b6 b7 b8 b9\};

$a=\operatorname{sum}(x 1) ; b=\operatorname{sum}(y 1) ; c=\operatorname{sum}(b 1) ; d=\operatorname{sum}(b 1 \# y 1+(1-b 1) \# x 1) ;$

print $\mathrm{x} 1 \mathrm{y} 1 \mathrm{~b} 1$; print $\mathrm{a} b \mathrm{~b}$;

$a=\operatorname{sum}(x 2) ; b=\operatorname{sum}(y 2) ; c=\operatorname{sum}(b 2) ; d=\operatorname{sum}(b 2 \# y 2+(1-b 2) \# x 2) ;$

print $\mathrm{x} 2$ y2 b2; print $\mathrm{a} b \mathrm{~b}$;

$a=\operatorname{sum}(x 3) ; b=\operatorname{sum}(y 3) ; c=\operatorname{sum}(b 3) ; d=\operatorname{sum}(b 3 \# y 3+(1-b 3) \# \times 3) ;$

print $\mathrm{x} 3$ y3 $\mathrm{b} 3$; print $\mathrm{a} b \mathrm{~b} d$;

$a=\operatorname{sum}(x 4) ; b=\operatorname{sum}(y 4) ; c=\operatorname{sum}(b 4) ; d=\operatorname{sum}(b 4 \# y 4+(1-b 4) \# x 4) ;$

print $\mathrm{x} 4 \mathrm{y} 4 \mathrm{~b} 4$; print $\mathrm{a} b \mathrm{~b}$;

$a=\operatorname{sum}(x 5) ; b=\operatorname{sum}(y 5) ; c=\operatorname{sum}(b 5) ; d=\operatorname{sum}(b 5 \# y 5+(1-b 5) \# x 5) ;$

print $\mathrm{x} 5$ y5 b5; print $\mathrm{a} b \mathrm{~b}$;

$a=\operatorname{sum}(x 6) ; b=\operatorname{sum}(y 6) ; c=\operatorname{sum}(b 6) ; d=\operatorname{sum}(b 6 \# y 6+(1-b 6) \# x 6) ;$ 
print $x 6$ y6 b6; print a b c d;

$a=\operatorname{sum}(x 7) ; b=\operatorname{sum}(y 7) ; c=\operatorname{sum}(b 7) ; d=\operatorname{sum}(b 7 \# y 7+(1-b 7) \# x 7) ;$

print $x 7$ y7 b7; print a b c d;

$a=\operatorname{sum}(x 8) ; b=\operatorname{sum}(y 8) ; c=\operatorname{sum}(b 8) ; d=\operatorname{sum}(b 8 \# y 8+(1-b 8) \# x 8) ;$

print $x 8$ y8 b8; print $a$ b c d;

$a=\operatorname{sum}(x 9) ; b=\operatorname{sum}(y 9) ; c=\operatorname{sum}(b 9) ; d=\operatorname{sum}(b 9 \# y 9+(1-b 9) \# x 9)$;

print $x 9$ y9 b9; print $a$ b c d;

\section{Programa 9}

Este programa é implementado para gerar a amostra G-MH isto é, para obter a amostra "Gibbs-com-Metropolis-Hastings" através da metodologia apresentada no Capítulo 8. Para obtenção de amostras G-MH necessárias para o desenvolvimento dos exemplos ilustrativos 2 e 3 modificações convenientes foram feitas no programa que segue. Este programa contém 3 macros a saber:

( i) MACROJ responsável por cálculos auxiliares para obtenção da amostra G$\mathrm{MH}$.

( ii) MACROH responsável pelo cálculo da probabilidade de transição do algoritmo de "Metropolis-Hastings".

(iii) MACroJ "Chama" a MACROK e a MACROH e realiza as atualizações necessárias nos parâmetros $c_{1}, c_{2}, c_{3}, \beta_{1}$ e $\beta_{3}$ considerando as densidades a posteriori condicionais cheias dadas pela expressão (8.9).

Iibname novo "r: I";

options ps $=10000$;

proc iml worksize $=15000$ symsize $=15000$; 
/* Escolher o numero da sequencia o qual varia de 1 ate $10 * /$

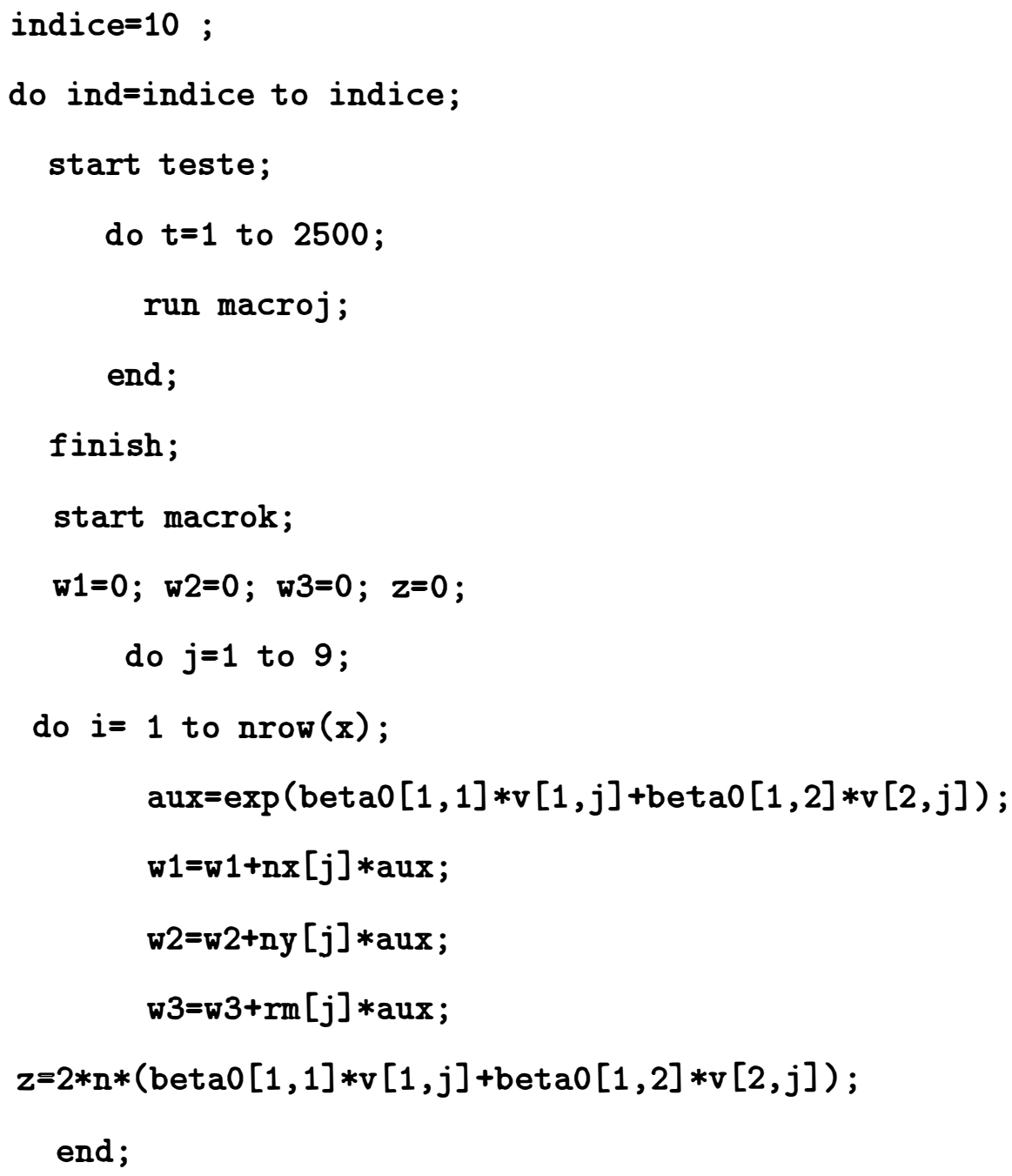


$\mathrm{p}=\mathrm{c} 1 / \mathrm{c} 12$;

Nm1 =ranbin (second, 4,p); / $*$ variavel latente $* /$

$\mathrm{c} 10$ =rangam (second, a1)/b1; /* Gerando um novo dado para $c 1 * /$ $c 1230=c 10+c 2+c 3 ;$

$c 130=c 10+c 3$;

$c 120=c 10+c 2$;

$\operatorname{beta} 0[1,1]=\operatorname{beta}[1,1]$;

$\operatorname{bet} a 0[1,2]=\operatorname{beta}[1,2]$;

run macrok;

$\mathrm{k} 24=(\mathrm{Nm} 1+\mathrm{r}) * \log (\mathrm{c} 10)+(\mathrm{n}-\mathrm{r}) * \log (\mathrm{c} 130)+\mathrm{n} * \log (\mathrm{c} 1230)$

$-(n-n 1) * \log (c 120)-c 10 * w 1 ;$

$c 123=c 1+c 2+c 3 ;$

$c 13=c 1+c 3$;

$c 12=c 1+c 2$;

beta $0[1,1]=\operatorname{bet} a[1,1]$;

beta $0[1,2]=\operatorname{bet} a[1,2]$;

run macrok;

$\mathrm{k} 25=(\mathrm{Nm} 1+\mathrm{r}) * \log (\mathrm{c} 1)+(\mathrm{n}-\mathrm{r}) * \log (\mathrm{c} 13)+\mathrm{n} * \log (\mathrm{c} 123)$

$-(n-n 1) * \log (c 12)-c 1 * w 1 ;$

run macroh;

$c 1=c 10 *$ bern $+c 1 *(1-$ bern $)$;

/* Se bern=1 altera-se o valor de c10; */

/* Se bern=0 o valor de $c 1$ permanece o mesmo */

if bern $=1$ then $\operatorname{cont} 1=(\operatorname{cont} 1+1)$;

/* indaceit $=$ indice de aceitacao do valor gerado $* /$

$c 20=$ rangam (second , a2/b2 ; 


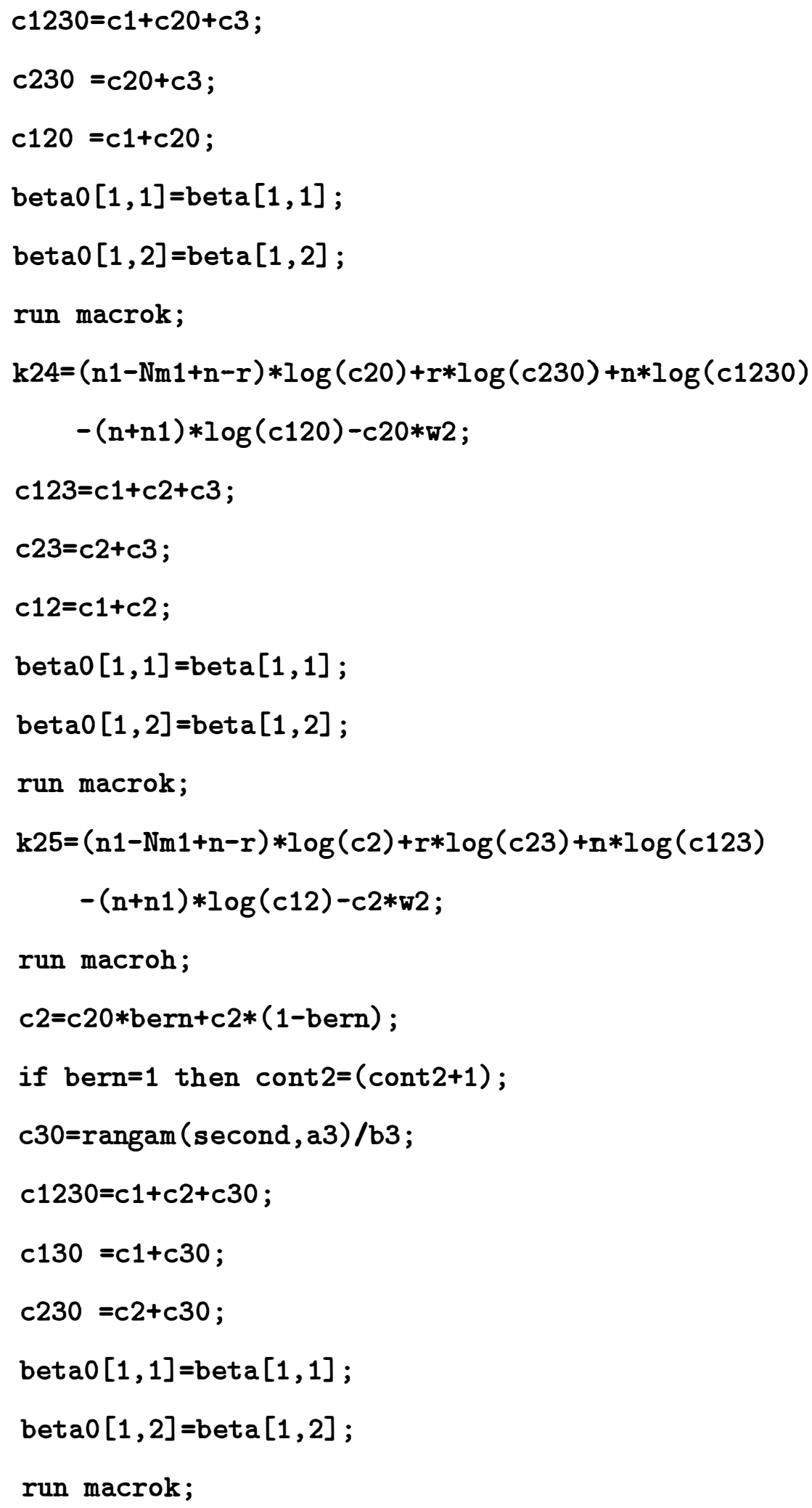


$\mathrm{k} 24=r * \log (c 230)+(n-r) * \log (c 130)+n * \log (c 1230)-c 30 * w 3 ;$

$c 123=c 1+c 2+c 3$

$c 13=c 1+c 3$

$c 23=c 2+c 3$

$\operatorname{beta} 0[1,1]=\operatorname{bet} a[1,1]$;

$\operatorname{beta} 0[1,2]=\operatorname{beta}[1,2]$;

run macrok;

$\mathrm{k} 25=r * \log (c 23)+(n-r) * \log (c 13)+n * \log (c 123)-c 3 * w 3 ;$

run macroh;

c3 $=c 30 *$ bern $+c 3 *(1-$ bern $)$;

if bern=1 then $\operatorname{cont} 3=(\operatorname{cont} 3+1)$;

betag=mu1+sigma $1 *$ rannor (second);

beta0 $[1,1]=$ betag;

$\operatorname{beta} 0[1,2]=\operatorname{beta}[1,2]$;

run macrok;

$k 24=2 * z-c 1 *(b 1+w 1)-c 2 *(b 2+w 2)-c 3 *(b 3+w 3)$;

$\operatorname{beta} 0[1,1]=\operatorname{beta}[1,1]$;

$\operatorname{beta} 0[1,2]=\operatorname{bet} a[1,2]$;

run macrok;

$\mathrm{k} 25=2 * \mathrm{z}-\mathrm{c} 1 *(\mathrm{~b} 1+\mathrm{w} 1)-\mathrm{c} 2 *(\mathrm{~b} 2+\mathrm{w} 2)-\mathrm{c} 3 *(\mathrm{~b} 3+\mathrm{w})$;

run macroh;

$\operatorname{beta}[1,1]=\operatorname{betag} * \operatorname{bern}+\operatorname{bet} a[1] *(1-\operatorname{bern})$;

betares $1=\operatorname{beta}[1,1]$;

if bern=1 then cont $4=(\operatorname{cont} 4+1)$;

betag $=$ mu $2+$ sigma $2 *$ rannor $($ second $)$;

beta $0[1,2]=$ betag ; 


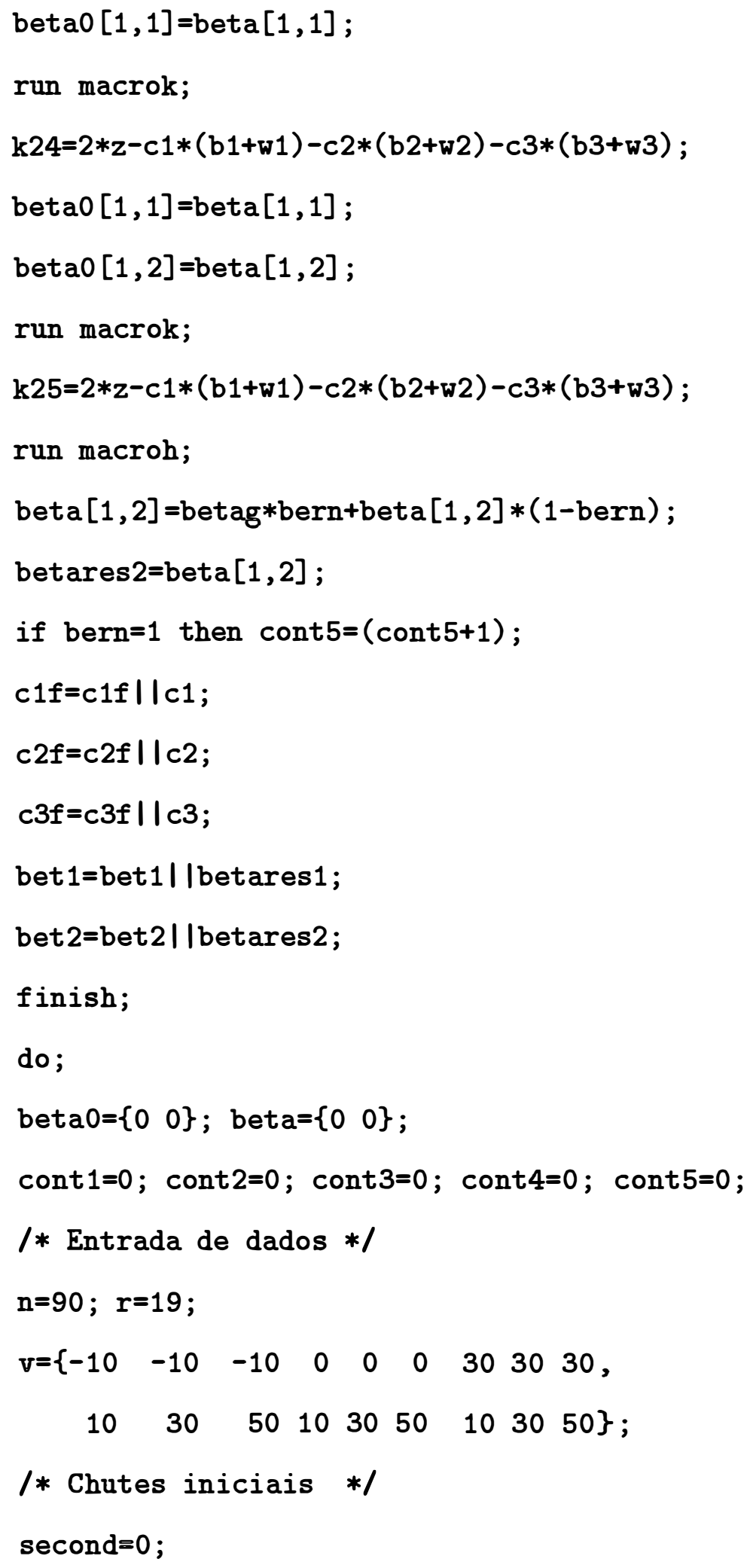




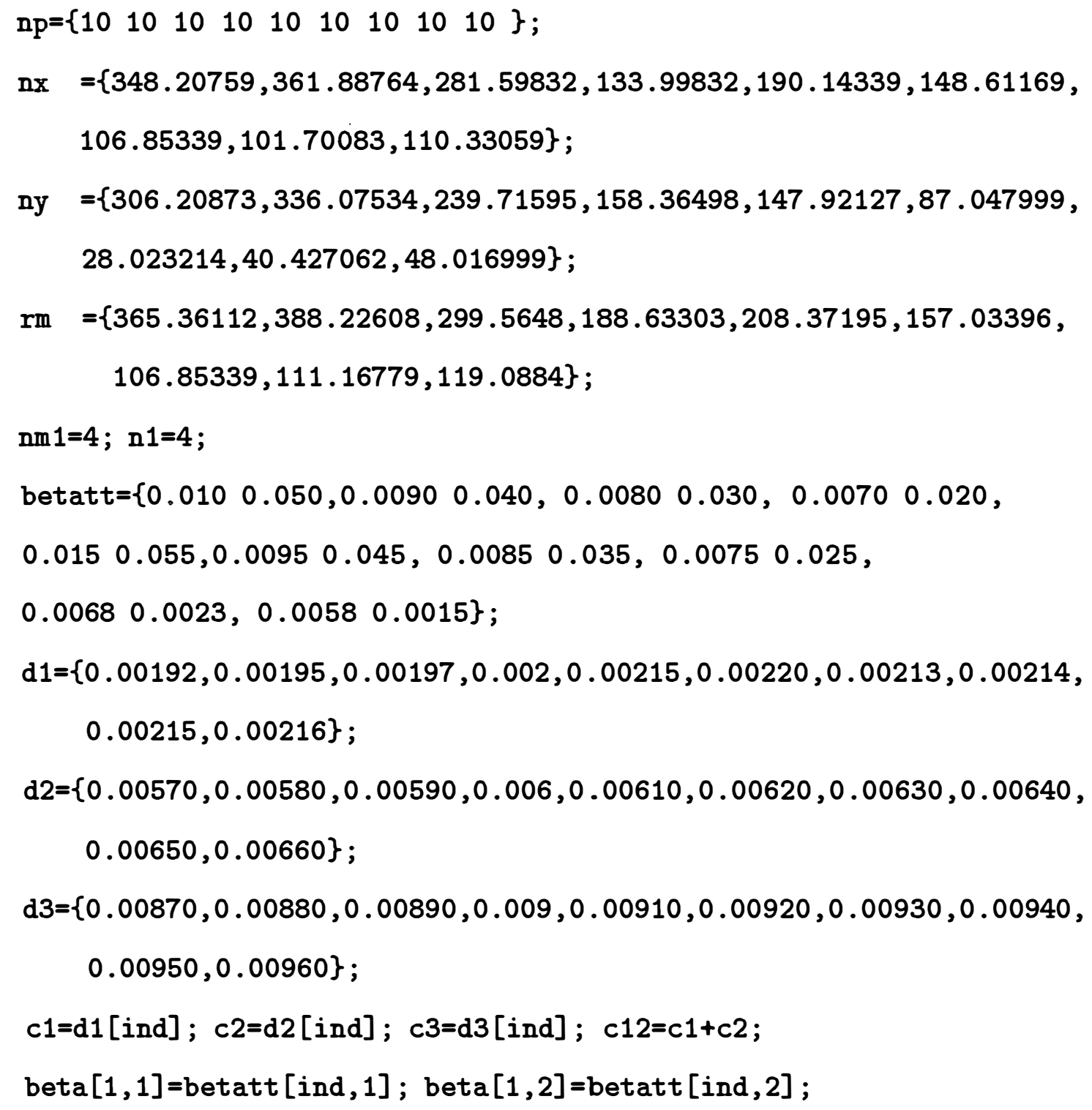


a3=c3f';

a4=bet ${ }^{\prime}$;

a5=bet $2^{\prime}$;

arq1=a1 | | a2 | | a3 | |a4 | la5;

$r=\{c 1$ c2 c3 beta1 beta2 $\}$;

create novo.arq1 from arq1 [colname=r];

append from arq1;

end;

result $=$ shape $(0,100,6)$;

in $=0$;

do $m=1000$ to 2500 by 10 ;

in $=i n+1$;

if in $>100$ then goto $\mathrm{fim}$;

result $[$ in, 1$]=i n$;

result $[$ in , 2] $=\mathrm{a} 1[\mathrm{~m}, 1]$;

result $[$ in , 3] $=a 2[\mathrm{~m}, 1]$;

result $[$ in , 4] $=\mathrm{a} 3[\mathrm{~m}, 1]$;

result $[$ in , 5] $=\mathrm{a} 4[\mathrm{~m}, 1]$;

result $[$ in, 6$]=a 5[\mathrm{~m}, 1]$;

fim:

end ;

print result;

arq16=result $[, 1]||$ result $[, 2]||$ result $[, 3]||$ result $[, 4]$

| |result $[, 5]$ | |result $[, 6]$;

$r 1=\{$ in $c 1$ c2 c3 beta1 beta2 $\}$;

create novo.arq16 from arq16[colname=r1]; 
append from arq16;

$\operatorname{ss} 2=\operatorname{sum}(\operatorname{result}[, 2]) / \operatorname{nrow}(\operatorname{result}[, 2])$;

ss3=sum $($ result $[, 3]) /$ nrow $($ result $[, 3])$;

ss4=sum $(\operatorname{result}[, 4]) / \operatorname{nrow}(\operatorname{result}[, 4])$;

ss5 $=\operatorname{sum}($ result $[, 5]) /$ nrow $($ result $[, 5])$;

ss6 $=\operatorname{sum}($ result $[, 6]) /$ nrow $($ result $[, 6])$;

print ss2 ss3 ss4 ss5 ss6;

print cont 1 cont 2 cont 3 cont 4 cont5;

quit;

data_null_;

set novo.arq16;

file "k: lout \tese10.txt";

format in c1 c2 c3 beta1 beta2 7.5;

put 01 in 99 c1 $\$ 17$ c2 $@ 25$ c3 $\$ 33$ beta1 $\$ 42$ beta2;

run;

\section{Programa 10}

Este programa foi implementado no "software" SPLuS e tem como objetivo calcular o valor de $\hat{R}$, segundo o critério de GELMAN \& RUBIN, necessário para monitorar a convergência da amostra G-MH obtida no Capítulo 8.

S-PLUS : Copyright 1988, 1995 MathSoft, Inc.

$S$ : Copyright AT\&T.

Version 3.3 Release 1 for MS Windows 3.1 : 1995

Working data will be in $r: l_{-}$Data

$>$ monitor<-source("k: \Igelman\lmonitor.sps")

$>$ col.means<-source ("k: IIgelman IIcolmeans.sps") 
$>$ col.vars<-source("k: Mgelman Icolvars.sps")

$>$ cov<-source("k: |Igelman \/cov.sps")

$>$ chisqdf<-source("k: Mgelman Mchisqdf.sps")

$>$ gpar<-source("k: \Igelman \gpar.sps")

> r<-matrix(scan("k: Mout \tese55.txt"), byrow=T, ncol=6)

$>r<-r[, 2: 6]$

$>a<-a s \cdot \operatorname{vector}(r)$

$>\operatorname{dim}(a)<-c(100,10,5)$

$>c 1<-a[,, 1]$

$>c 2<-a[,, 2]$

$>\mathrm{c} 3<-\mathrm{a}[,, 3]$

$>$ betal<-a $[,, 4]$

$>$ beta $2<-a[,, 5]$

$>$ win.graph ()

$>\operatorname{par}(\operatorname{mfrow}=c(2,3))$

$>\operatorname{par}(p t y=" s ")$

$>$ monitor(a)

$>$ summary.c1<-summary (c1)

$>$ summary.c2<-summary (c2)

$>$ summary.c3<-summary (c3)

$>$ summary.beta1<-summary (beta1)

$>$ summary.beta2<-summary (beta2)

$>$ iqd1<-summary.c1[5]-summary.c1[2]

$>$ iqd2<-summary.c2[5]-summary .c2 [2]

$>$ iqd3<-summary.c3[5]-summary.c3[2]

$>$ iqd4<-summary.beta1 [5]-summary.beta1 [2] 
$>$ iqd5<-summary $\cdot$ beta2 [5] -summary $\cdot$ beta2 [2]

$>\operatorname{plot}(\operatorname{density}(c 1$, width=2*iqd1), type="l", bty="l", $\mathrm{xlab}=" \mathrm{C} 1 "$, ylab="VER")

$>$ plot(density (c2, width=2*iqd2), type="l", bty="1", $x l a b=" C 2 "$, $\mathrm{ylab}=$ "VER")

$>$ plot (density (c3, width=2*iqd3), type="l", bty="l", xlab="C3", $\mathrm{ylab}=$ "VER")

$>$ plot (density (beta1, width=2*iqd4), type="l", bty="l", xlab="BETA .1", ylab="VER")

$>$ plot (density (beta2, width=2*iqd5), type="l", bty="l", $\mathrm{xlab}="$ BETA.2", ylab="VER")

$>$ postscript("r:Mregc1.ps")

$>\operatorname{plot}(\operatorname{density}(c 1$, width=2*iqd1), type="l", bty="l", xlab="C1" ,ylab="VER")

$>\operatorname{dev}$. off ()

win.graph

2

$>$ postscript("r: /lregc2.ps")

$>$ plot (density (c2, width=2*iqd2), type="l" , bty="1" , xlab="C2", ylab="VER")

$>\operatorname{dev} . \operatorname{off}()$

win.graph

2

$>$ postscript("r:Mregc3.ps")

$>\operatorname{plot}(\operatorname{den} \operatorname{sity}(c 3$, width=2*iqd3), type="l", bty="l", $x l a b=" C 3 "$, $\mathrm{yl} a \mathrm{ab}=$ "VER") 
$>\operatorname{dev} \cdot \operatorname{off}()$

win.graph

2

> postscript("r: Mregbeta1.ps")

$>$ plot (density (beta1, width=2*iqd4), type="l", bty="l", $\mathrm{xlab}=$ "BETA.1", ylab="VER")

$>\operatorname{dev} \cdot \operatorname{off}()$

win.graph

2

> postscript("r:|Mregbeta2.ps")

$>$ plot (density (beta2, width=2*iqd5), type="l" , bty="l", $x l a b=" B E T A .2 ", y l a b=" V E R ")$

$>\operatorname{dev}$.off ()

win.graph

2 
Supondo que

$$
\left[\begin{array}{lll}
i_{11} & i_{12} & i_{13} \\
i_{21} & i_{22} & i_{23} \\
i_{31} & i_{32} & i_{33}
\end{array}\right]
$$

represente a matriz de informação observada e sabendo-se que esta matriz é simétrica. Temos que:

1) Os elementos da matriz de informação observada considerando-se a reparametrização:

$\left(\lambda_{1}, \lambda_{2}, \lambda_{3}\right)$ são dados por:

$$
\begin{aligned}
& i 11=\frac{r}{\lambda_{1}^{2}}+\frac{n}{\left(\lambda_{1}+\lambda_{2}+\lambda_{3}\right)^{2}}+\frac{n}{\left(\lambda_{1}+\lambda_{3}\right)^{2}}-\frac{r}{\left(\lambda_{1}+\lambda_{3}\right)^{2}}-\frac{n}{\left(\lambda_{1}+\lambda_{2}\right)^{2}} \\
& i 12=\frac{n}{\left(\lambda_{1}+\lambda_{2}+\lambda_{3}\right)^{2}}-\frac{n}{\left(\lambda_{1}+\lambda_{2}\right)^{2}}
\end{aligned}
$$$$
i 13:=\frac{n}{\left(\lambda_{1}+\lambda_{2}+\lambda_{3}\right)^{2}}+\frac{n}{\left(\lambda_{1}+\lambda_{3}\right)^{2}}-\frac{r}{\left(\lambda_{1}+\lambda_{3}\right)^{2}}
$$$$
i 22=\frac{n}{\left(\lambda_{1}+\lambda_{2}+\lambda_{3}\right)^{2}}+\frac{n}{\lambda_{2}{ }^{2}}-\frac{r}{\lambda_{2}{ }^{2}}+\frac{r}{\left(\lambda_{2}+\lambda_{3}\right)^{2}}-\frac{n}{\left(\lambda_{1}+\lambda_{2}\right)^{2}}
$$

$$
i 23=\frac{n}{\left(\lambda_{1}+\lambda_{2}+\lambda_{3}\right)^{2}}+\frac{r}{\left(\lambda_{2}+\lambda_{3}\right)^{2}}
$$

$$
33=\frac{n}{\left(\lambda_{1}+\lambda_{2}+\lambda_{3}\right)^{2}}+\frac{r}{\left(\lambda_{2}+\lambda_{3}\right)^{2}}+\frac{n}{\left(\lambda_{1}+\lambda_{3}\right)^{2}}-\frac{r}{\left(\lambda_{1}+\lambda_{3}\right)^{2}}
$$


2) Os elementos da matriz de informação observada considerando-se a reparametrização: 170 $\left(\theta_{1}, \theta_{2}, \theta_{3}\right)$ são dados por:

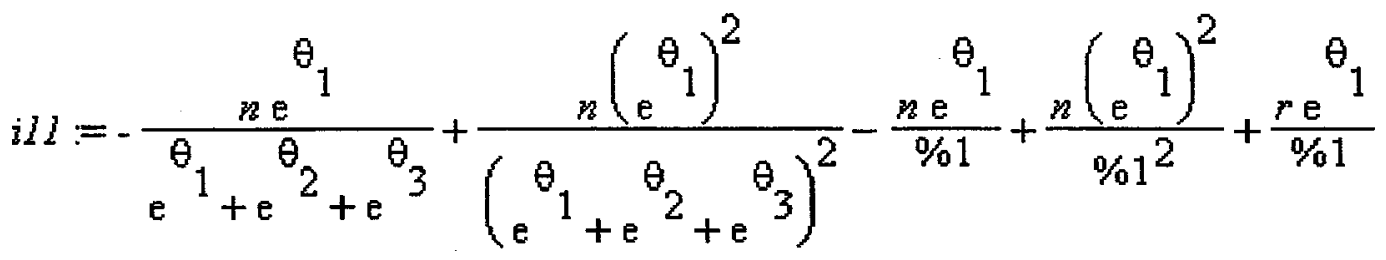

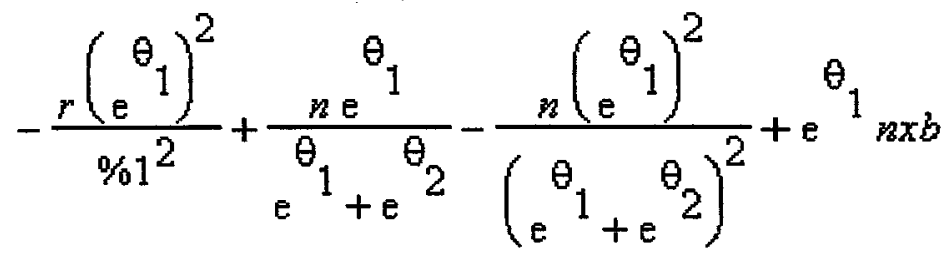

$$
\begin{aligned}
& \% 1=e^{\theta_{1}}+e^{\theta_{3}}
\end{aligned}
$$

$i 12=\frac{n \mathrm{e}^{\theta_{1}} \mathrm{e}^{\theta_{2}}}{\left(\mathrm{e}^{\theta_{1}}+\mathrm{e}^{\theta_{2}}+\mathrm{e}^{\theta_{3}}\right)^{2}}-\frac{n \mathrm{e}^{\theta_{1}} \mathrm{e}^{\theta_{2}}}{\left(\mathrm{e}^{\theta_{1}}+\mathrm{e}^{\theta_{2}}\right)^{2}}$

$i l 3=\frac{n \mathrm{e}^{\theta_{1}} \mathrm{e}^{\theta_{3}}}{\left(\mathrm{e}^{\theta_{1}}+\mathrm{e}^{\theta_{2}}+\mathrm{e}^{\theta_{3}}\right)^{2}}+\frac{n \mathrm{e}^{\theta_{1}} \mathrm{e}^{\theta_{3}}}{\left(\mathrm{e}^{\theta_{1}}+\mathrm{e}^{\theta_{3}}\right)^{2}}-\frac{r \mathrm{e}^{\theta_{1}} \mathrm{e}^{\theta_{3}}}{\left(\mathrm{e}^{\theta_{1}}+\mathrm{e}^{\theta_{3}}\right)^{2}}$ 


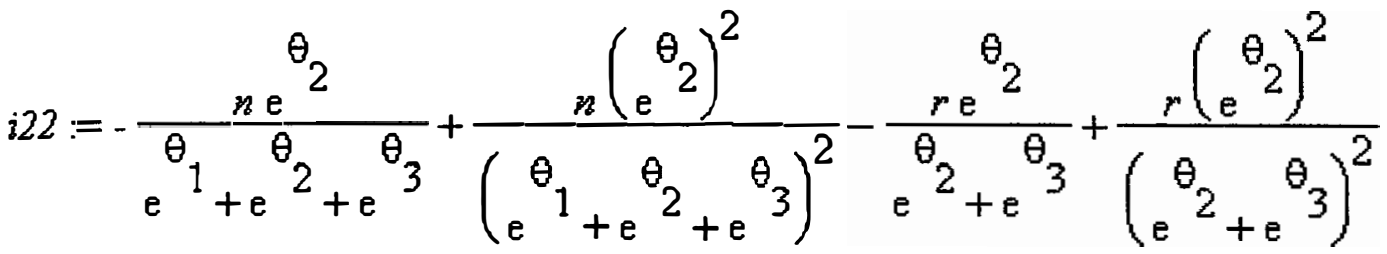

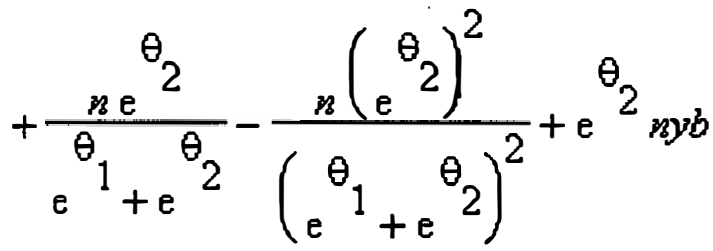

$$
\begin{aligned}
& i 23=\frac{n \mathrm{e}^{\theta_{2}} \mathrm{e}^{\theta_{3}}}{\left(\mathrm{e}^{\theta_{1}}+\mathrm{e}^{\theta_{2}}+\mathrm{e}^{\theta_{3}}\right)^{2}}+\frac{r_{\mathrm{e}^{2}} \mathrm{e}^{\theta^{3}}}{\left(\mathrm{e}^{\theta_{2}}+\mathrm{e}^{\theta_{3}}\right)^{2}} \\
& i 33=-\frac{n \mathrm{e}^{\theta_{3}}}{\mathrm{e}^{\theta_{1}}+\mathrm{e}^{\theta_{2}}+\mathrm{e}^{\theta_{3}}}+\frac{n\left(\mathrm{e}^{3}\right)^{2}}{\left(\mathrm{e}^{\theta_{1}}+\mathrm{e}^{\theta_{2}}+\mathrm{e}^{\theta_{3}}\right)^{2}}-\frac{r^{\theta^{3}}}{\mathrm{e}^{\theta_{2}}+\mathrm{e}^{\theta_{3}}}+\frac{r\left(\mathrm{e}^{3}\right)^{2}}{\left(\mathrm{e}^{\theta_{2}}+\mathrm{e}^{\theta_{3}}\right)^{2}}-\frac{\mathrm{e}^{3}}{\% 1} \\
& +\frac{n\left(\mathrm{e}^{3}\right)^{2}}{\% 1^{2}}+\frac{r \mathrm{e}^{\theta_{3}}}{\% 1}-\frac{r\left(\mathrm{e}^{\theta_{3}}\right)^{2}}{\% 1^{2}}+\mathrm{e}^{3} R m \\
& \% 1=e^{\theta_{1}}+e^{\theta_{3}}
\end{aligned}
$$

3) Os elementos da matriz de informação observada na parametrização $\left(\phi_{1}, \phi_{2}, \phi_{3}\right)$, ou seja, considerando-se a transformação de Box \& Cox e supondo que $\xi_{i}$ é diferente de zero para $i=1,2,3$, são dados por: 


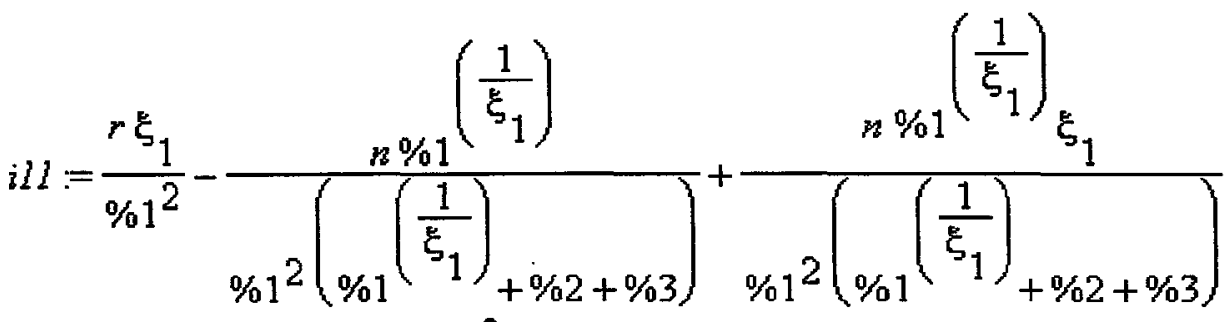

$$
\begin{aligned}
& +\frac{n(\% 1}{\left.\left.\% 1^{2}\left(\frac{1}{\xi_{1}}\right)\right)^{2}\left(\frac{1}{\xi_{1}}\right)_{+\% 2+\% 3}\right)^{2}}-\frac{n \% 1\left(\frac{1}{\xi_{1}}\right)}{\left.\left(\frac{1}{\xi_{1}}\right)_{+\% 3}\right)}
\end{aligned}
$$

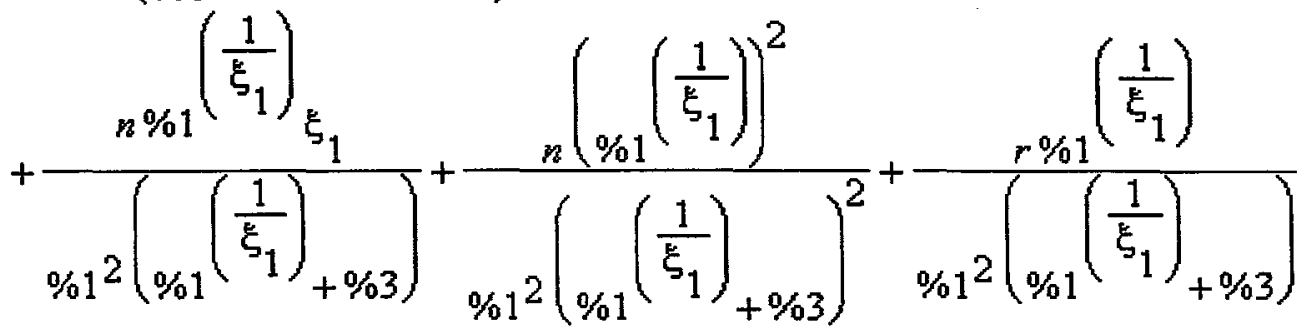

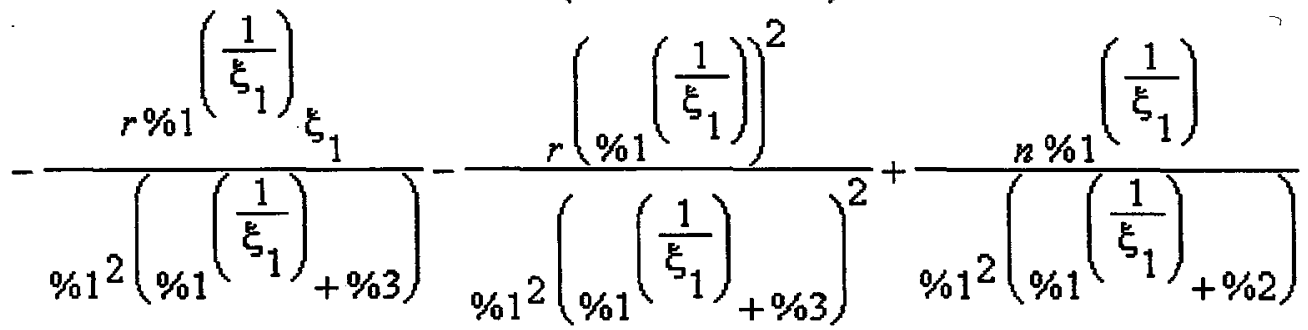

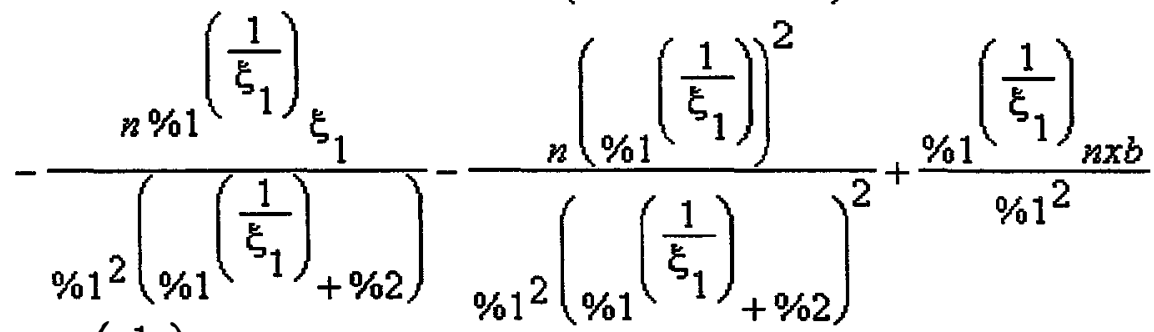

$$
\begin{aligned}
& -\frac{\%_{1}\left(\frac{1}{\xi_{1}}\right)_{n \pi b} \xi_{1}}{\% 1^{2}} \\
& \% 1:=1+\xi_{1} \phi_{1} \\
& \% 2:=\left(1+\xi_{2} \phi_{2}\right)^{\left(\frac{1}{\xi_{2}}\right)} \\
& \% 3:=\left(1+\xi_{3} \phi_{3}\right)^{\left(\frac{1}{\xi_{3}}\right)}
\end{aligned}
$$




$$
\begin{aligned}
& j 12:=\frac{n \% 2^{\left(\frac{1}{\xi_{1}}\right)}\left(\frac{1}{\xi_{2}}\right)}{\left(\frac{1}{\left(\frac{1}{\xi_{1}}\right)}+\left(\frac{1}{\xi_{2}}\right)+\left(1+\xi_{3} \phi_{3}\right)^{\left.\left(\frac{1}{\xi_{3}}\right)\right)^{2} \% 1}\right.} \\
& -\frac{n \% 2\left(\frac{1}{\xi_{1}}\right)_{\% 1}\left(\frac{1}{\xi_{2}}\right)}{\left.\left(\frac{1}{\xi_{1}}\right){ }_{+\% 1}\left(\frac{1}{\xi_{2}}\right)\right)_{\% 1}^{2}} \\
& \% 1=1+\xi_{2} \phi_{2} \\
& \% 2:=1+\xi_{1} \phi_{1}
\end{aligned}
$$

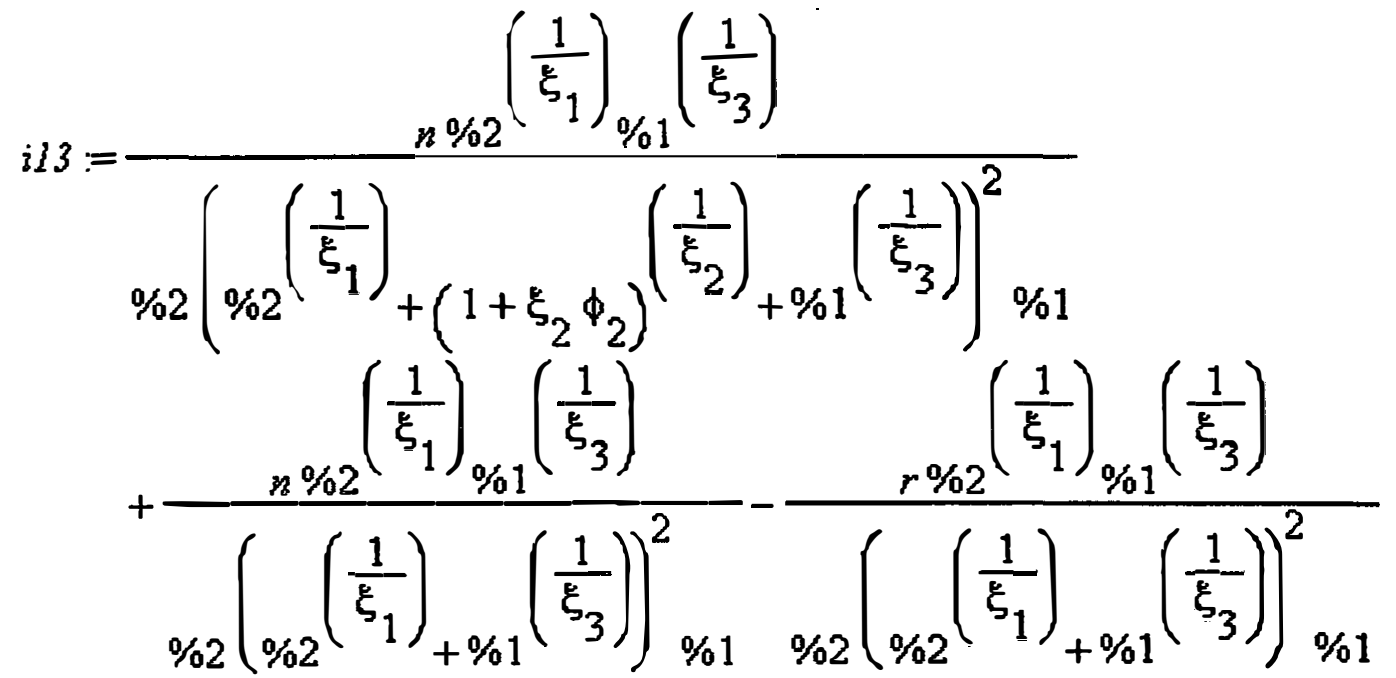

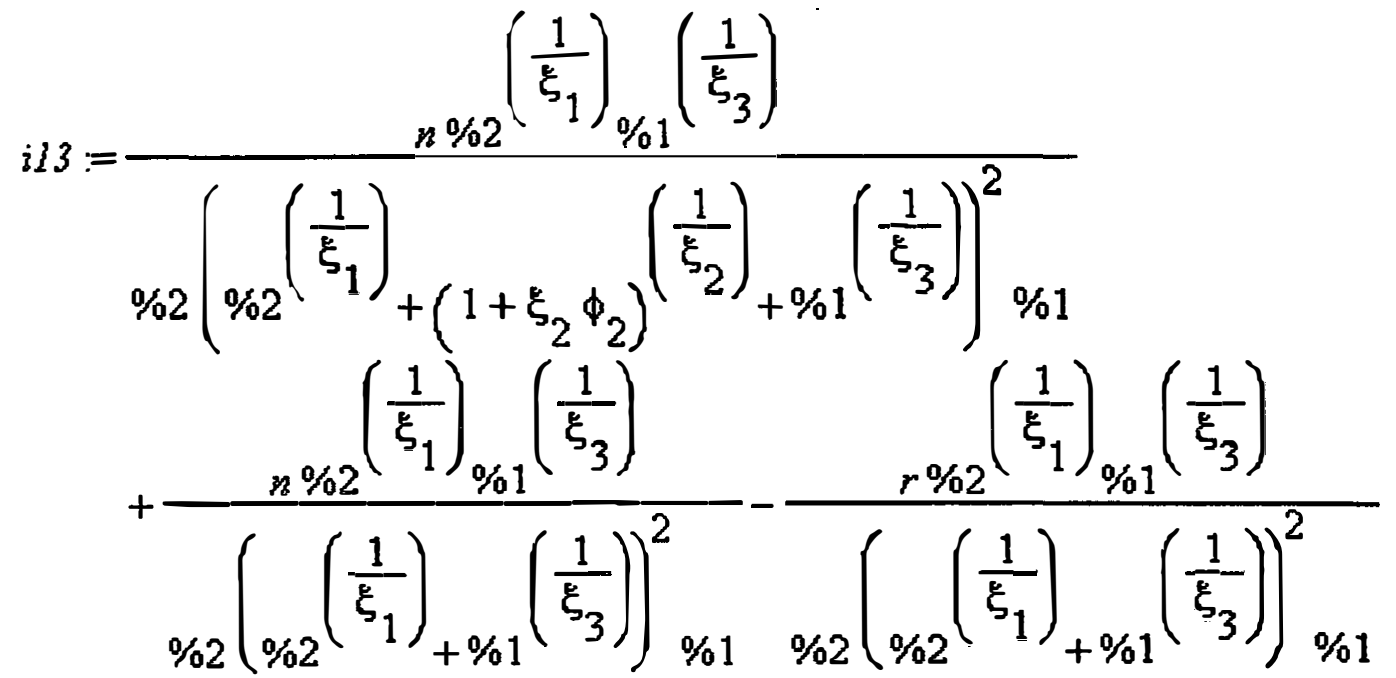

$$
\begin{aligned}
& \% 1:=1+\xi_{3} \phi_{3} \\
& \% 2:=1+\xi_{1} \phi_{1}
\end{aligned}
$$




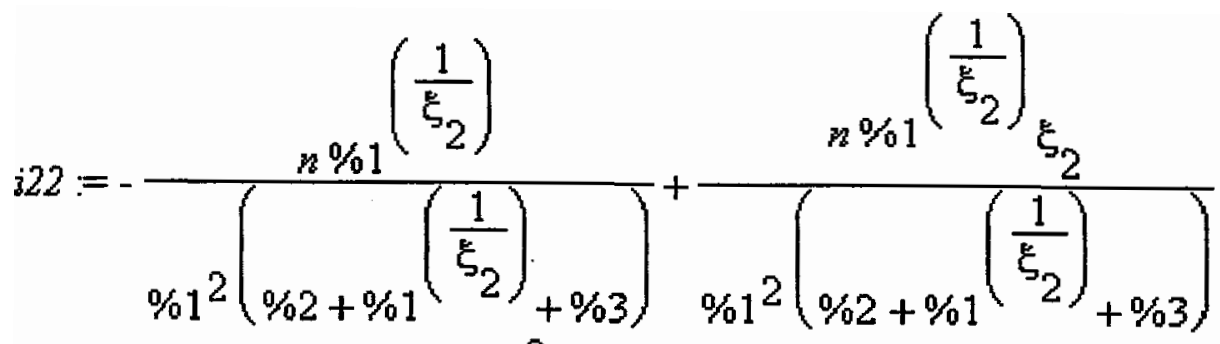

$$
\begin{aligned}
& +\frac{n\left(\% 1\left(\frac{1}{\xi_{2}}\right)\right)^{2}}{\left.\% 1^{2}\left(\frac{1}{\xi_{2}}\right){ }^{2}\right)^{2}+\frac{\xi_{2}}{\% 1^{2}}-\frac{r \xi_{2}}{\% 1^{2}}-\frac{r \% 1}{\left(\frac{1}{\xi_{2}}\right)}\left(\frac{1}{\left(\frac{1}{\xi_{2}}\right)}+\% 31^{2}\right)}
\end{aligned}
$$

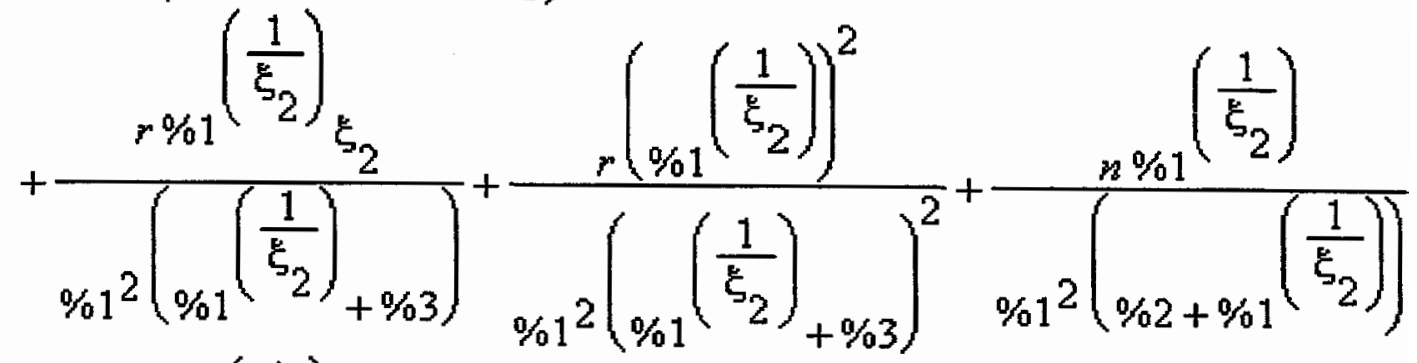

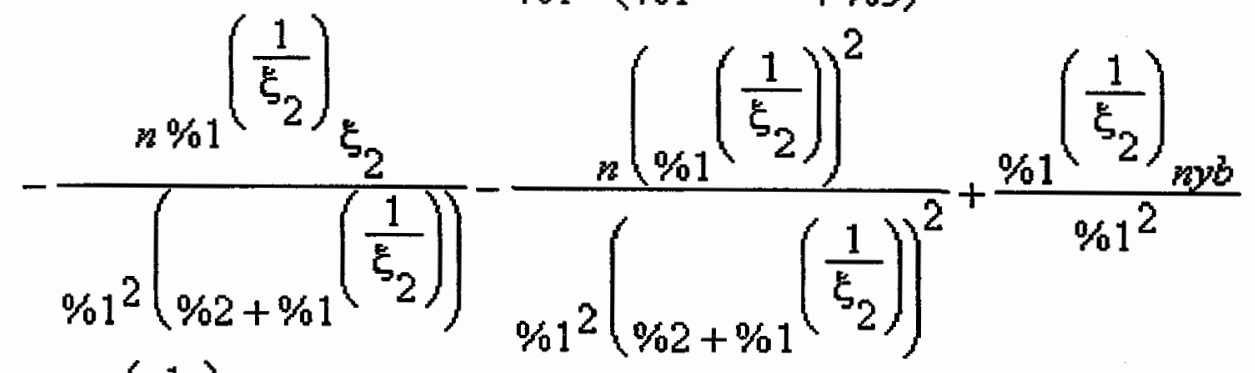

$$
\begin{aligned}
& -\frac{\% 1\left(\frac{1}{\xi_{2}}\right)}{\% y b \xi_{2}} \\
& \% 1:=1+\xi_{2} \phi_{2} \\
& \% 2:=\left(1+\xi_{1} \phi_{1}\right)^{\left(\frac{1}{\xi_{1}}\right)} \\
& \% 3=\left(1+\xi_{3} \phi_{3}\right)^{\left(\frac{1}{\xi_{3}}\right)}
\end{aligned}
$$




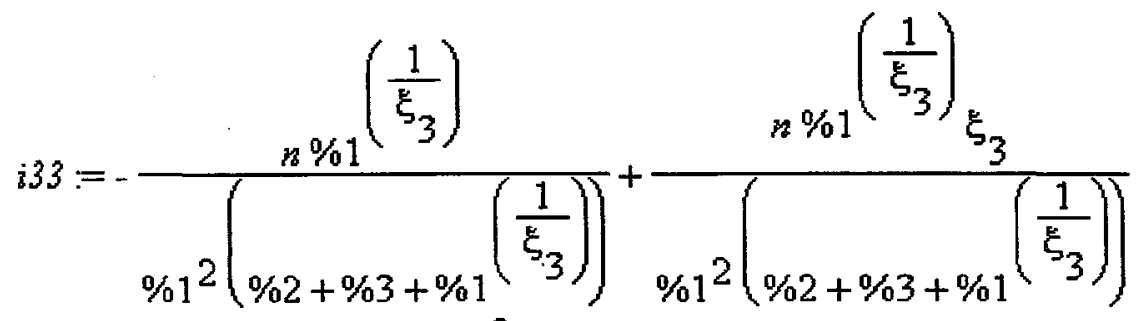

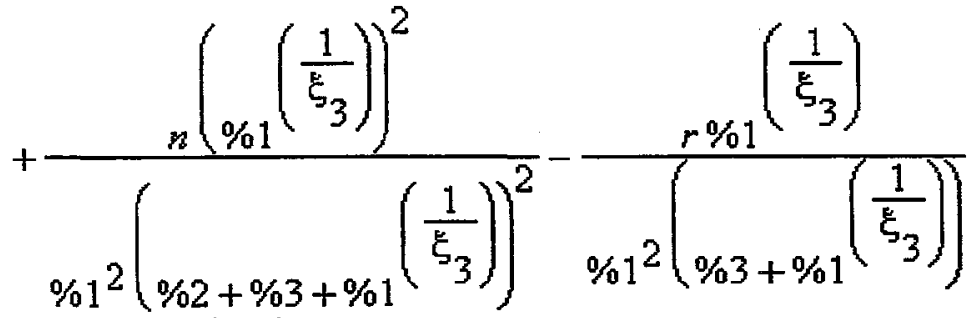

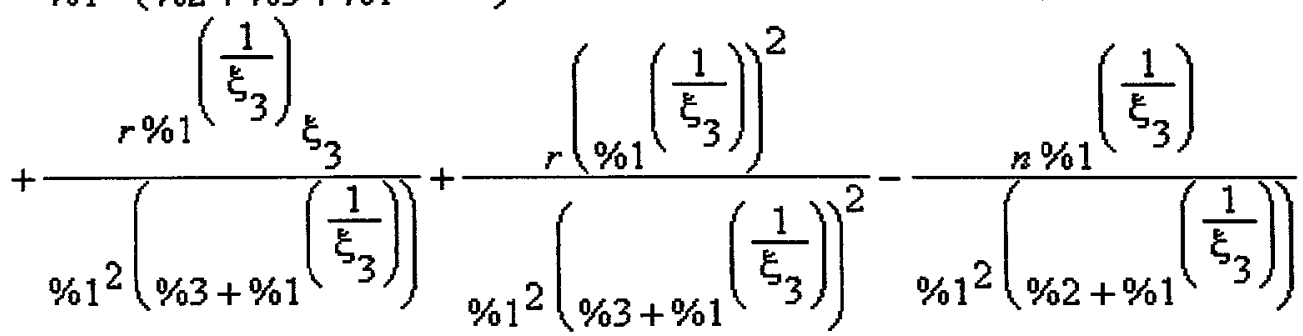

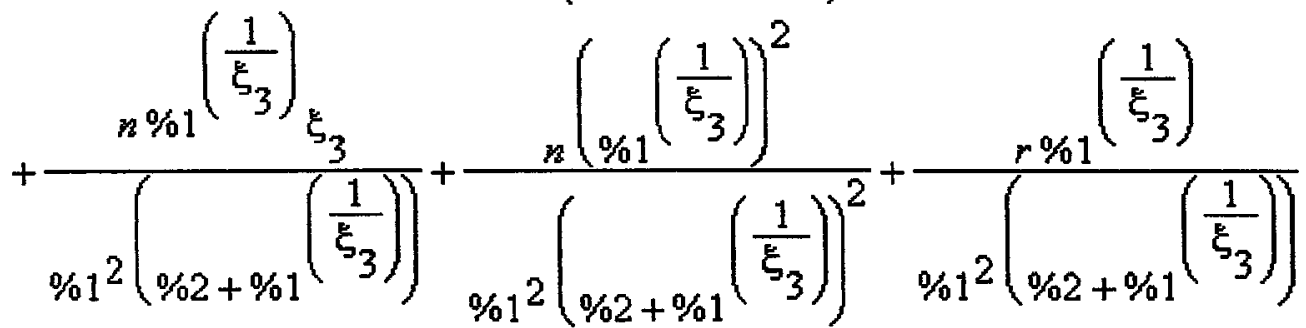

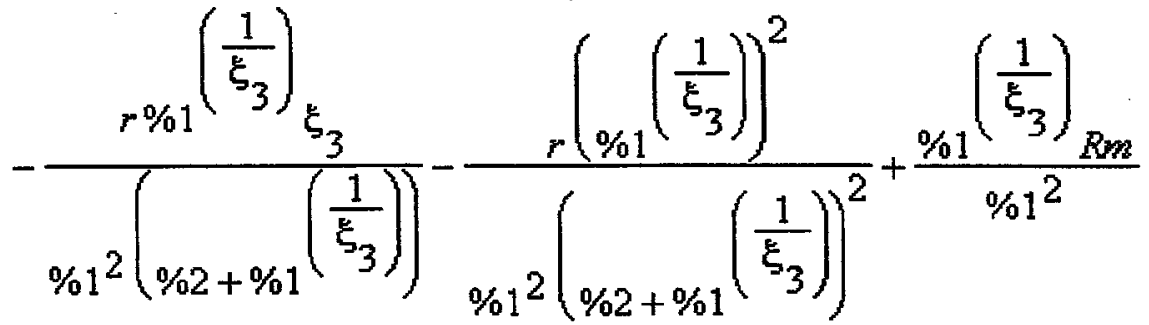

$$
\begin{aligned}
& -\frac{\left.\%_{1}^{\left(\frac{1}{\xi_{3}}\right.}\right)_{R m \xi_{3}}}{\% 1^{2}} \\
& \% 1:=1+\xi_{3} \phi_{3} \\
& \% 2:=\left(1+\xi_{1} \phi_{1}\right)^{\left(\frac{1}{\xi_{1}}\right)} \\
& \% 3:=\left(1+\xi_{2} \phi_{2}\right)^{\left(\frac{1}{\xi_{2}}\right)}
\end{aligned}
$$




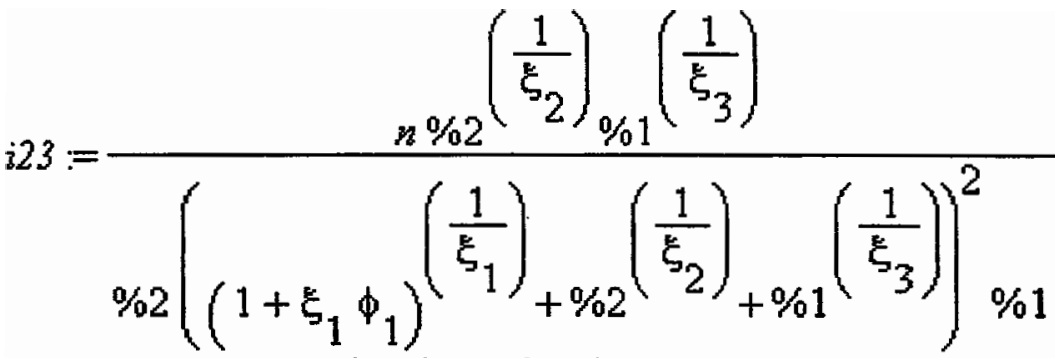

$$
\begin{aligned}
& +\frac{r \% 2\left(\frac{1}{\xi_{2}}\right)_{\% 1}\left(\frac{1}{\xi_{3}}\right)}{\left.\% 2\left(\frac{1}{\xi_{2}}\right)_{+\% 1}\left(\frac{1}{\xi_{3}}\right)\right)_{\% 1}^{2}} \\
& \% 1=1+\xi_{3} \phi_{3} \\
& \% 2=1+\xi_{2} \phi_{2}
\end{aligned}
$$

176

4) Os elementos da matriz de informação observada considerando-se a reparametrização: $\left(\lambda_{1}, \lambda_{2}, \theta_{0}\right)$ são dados por:

$$
\begin{aligned}
i 12= & \frac{n}{\theta_{0}{ }^{2} \ln \left(\theta_{0}\right)}+\frac{n}{\theta_{0}{ }^{2} \ln \left(\theta_{0}\right)^{2}}-\frac{r}{\theta_{0}{ }^{2} t_{0}\left(-\frac{\ln \left(\theta_{0}\right)}{t_{0}}-\lambda_{1}\right)}+\frac{r}{\theta_{0}{ }^{2} t_{0}{ }^{2}\left(-\frac{\ln \left(\theta_{0}\right)}{t_{0}}-\lambda_{1}\right)^{2}} \\
& -\frac{n}{\theta_{0}{ }^{2} t_{0} \% 1}+\frac{n}{\theta_{0}{ }^{2} t_{0}{ }^{2} \% 1^{2}}+\frac{r}{\theta_{0}{ }^{2} t_{0} \% 1}-\frac{r}{\theta_{0}{ }^{2} t_{0}{ }^{2} \% 1^{2}}+\frac{R m}{\theta_{0}{ }^{2} t_{0}} \\
\% 1:= & -\frac{\ln \left(\theta_{0}\right)}{t_{0}}-\lambda_{2} \\
i 12= & \frac{r}{\theta_{0} t_{0}\left(-\frac{\ln \left(\theta_{0}\right)}{t_{0}}-\lambda_{1}\right)^{2}}
\end{aligned}
$$




$$
\begin{aligned}
& i 13=\frac{n}{\theta_{0} t_{0}\left(-\frac{\ln \left(\theta_{0}\right)}{t_{0}}-\lambda_{2}\right)^{2}}-\frac{r}{\theta_{0} t_{0}\left(-\frac{\ln \left(\theta_{0}\right)}{t_{0}}-\lambda_{2}\right)^{2}} \\
& i 22=\frac{r}{\lambda_{1}^{2}}+\frac{r}{\left(-\frac{\ln \left(\theta_{0}\right)}{t_{0}}-\lambda_{1}\right)^{2}}-\frac{n}{\left(\lambda_{1}+\lambda_{2}\right)^{2}} \\
& i 23=-\frac{n}{\left(\lambda_{1}+\lambda_{2}\right)^{2}} \\
& i 33=\frac{n}{\lambda_{2}{ }^{2}}-\frac{r}{\lambda_{2}{ }^{2}}+\frac{n}{\left(-\frac{\ln \left(\theta_{0}\right)}{t_{0}}-\lambda_{2}\right)^{2}}-\frac{r}{\left(-\frac{\ln \left(\theta_{0}\right)}{t_{0}}-\lambda_{2}\right)^{2}}-\frac{n}{\left(\lambda_{1}+\lambda_{2}\right)^{2}}
\end{aligned}
$$

5) Os elementos da matriz de informação observada considerando-se a reparametrização:

$\left(\lambda_{1}, \lambda_{2}, \theta_{\mathrm{GJ}}\right)$ são dados por:

$$
\begin{aligned}
& i 12=r\left(\frac{(\xi \phi+1)\left(\frac{1}{\xi}\right)}{(\xi \phi+1)\left((\xi \phi+1)\left(\frac{1}{\xi}\right)+1\right)}-\frac{\left((\xi \phi+1)\left(\frac{1}{\xi}\right)\right)^{2}}{\left((\xi \phi+1)\left(\frac{1}{\xi}\right)+1\right)^{2}(\xi \phi+1)}\right) \\
& \left.\left.\left((\xi \phi+1)\left(\frac{1}{\xi}\right)_{+1}\right) /(\xi \phi+1)^{\left(\frac{1}{\xi}\right)_{t_{0}}\left(-\frac{\ln \left(\frac{(\xi \phi+1)}{(\xi \phi+1)}\left(\frac{1}{\xi}\right)\right.}{t_{0}}\right)}-\lambda_{1}\right)\right)^{2}
\end{aligned}
$$




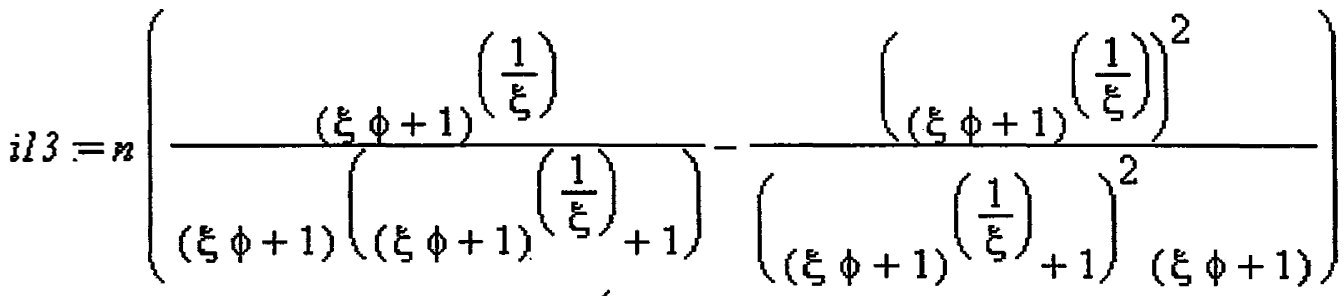

$$
\begin{aligned}
& \left({ }_{(\xi \phi+1)}\left(\frac{1}{\xi}\right)+1\right) /\left({ }_{(\xi \phi+1)}\left(\frac{1}{\xi}\right)_{t_{0}}\left(-\frac{\ln \left(\frac{(\xi \phi+1)}{(\xi \phi+1)}\left(\frac{1}{\xi}\right)\right.}{t_{0}}\right){ }^{\left(\frac{1}{\xi}\right)}-\lambda_{2}\right) \\
& -r\left(\frac{(\xi \phi+1)\left(\frac{1}{\xi}\right)}{(\xi \phi+1)\left((\xi \phi+1)\left(\frac{1}{\xi}\right)+1\right)}-\frac{\left({ }_{(\xi \phi+1)}\left(\frac{1}{\xi}\right)\right)^{2}}{\left({ }_{(\xi \phi+1)}\left(\frac{1}{\xi}\right)+1\right)^{2}}\right) \\
& \left.\left((\xi \phi+1)\left(\frac{1}{\xi}\right)+1\right) /(\xi \phi+1)\left(\frac{1}{\xi}\right)_{t_{0}}\left(-\frac{\ln \left(\frac{(\xi \phi+1)\left(\frac{1}{\xi}\right)}{(\xi \phi+1)^{\left(\frac{1}{\xi}\right)}+1}\right)}{t_{0}}-\lambda_{2}\right)^{2}\right) \\
& \left.i 22=\frac{r}{\lambda_{1}^{2}}+\frac{r}{\left(-\frac{\ln \left(\frac{(\xi \phi+1)}{(\xi \phi+1)}\left(\frac{1}{\xi}\right)\right.}{t_{0}}\right)+1}\right)^{2}-\frac{n}{\left(\lambda_{1}+\lambda_{2}\right)^{2}} \\
& i 23=-\frac{n}{\left(\lambda_{1}+\lambda_{2}\right)^{2}}
\end{aligned}
$$




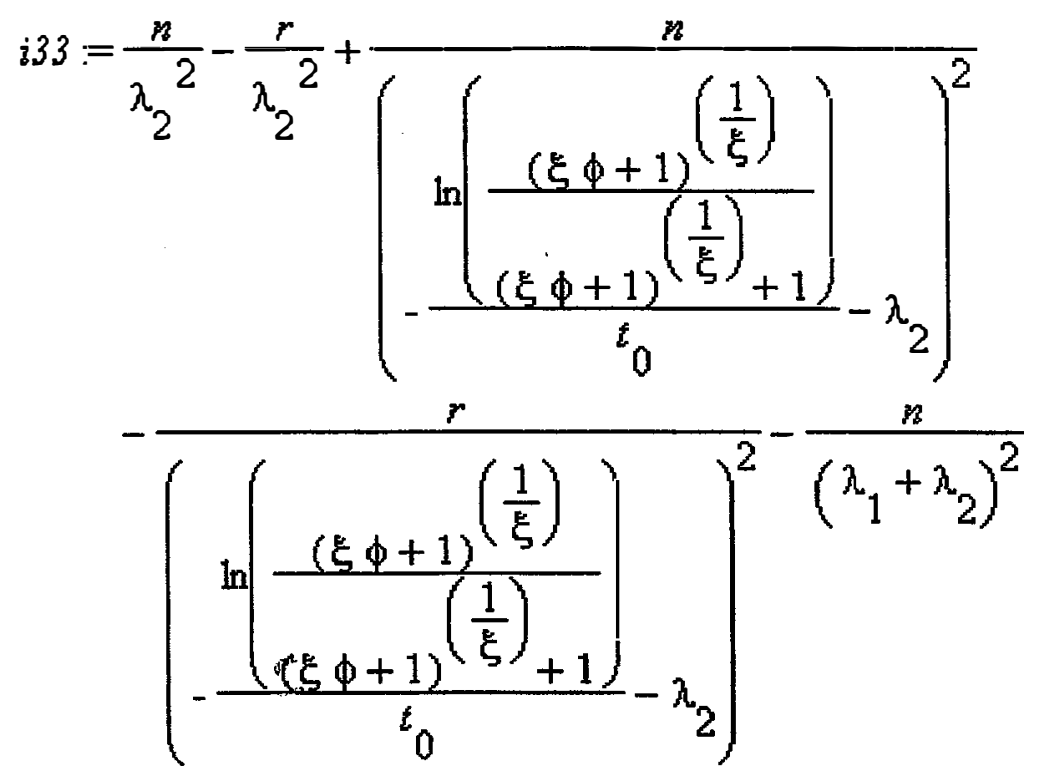

179 